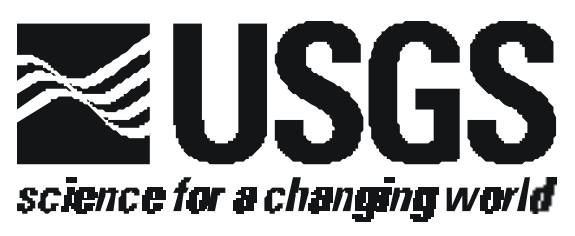

\title{
MAGNETOTELLURIC DATA ACROSS THE BATTLE MOUNTAIN-EUREKA AND CARLIN TRENDS, NORTH OF CORTEZ, NEVADA
}

\author{
by \\ Jackie M. Williams, Brian D. Rodriguez, \\ and \\ Douglas P. Klein1
}

Open-File Report 01-117

on-line edition

2001

This report is preliminary and has not been reviewed for conformity with U.S. Geological Survey editorial standards and stratigraphic nomenclature. Any use of trade, product, or firm names is for descriptive purposes only and does not imply endorsement by the U.S.

Government.

U.S. DEPARTMENT OF THE INTERIOR

U.S. GEOLOGICAL SURVEY

1 Denver, Colorado 
TABLE OF CONTENTS

INTRODUCTION

MAGNETOTELLURIC METHOD 3

MAGNETOTELLURIC SURVEY

MAGNETOTELLURIC DATA $\quad 6$

REFERENCES CITED 9

APPENDIX - MAGNETOTELLURIC DATA PLOTS 12 


\section{INTRODUCTION}

Genesis of gold deposits along the Battle Mountain-Eureka and Carlin trends in northern Nevada is not fully understood and subject to conflicting models (e.g. Arehart and others, 1993; Ilchik and Barton, 1997; Radtke, 1985; Shawe, 1991; Sillitoe and Bonham, 1990; Tosdal, 1998). A general consensus among these models is that regional structures somehow controlled the spatial distribution of the deposits. To investigate crustal structures that may be related to the genesis of gold deposits along these trends, a regional southwest-northeast profile of magnetotelluric (MT) soundings was acquired in 1994, 1996, 1997, and 2000 (1ine MT3-MT3", Figure 1). Resistivity modeling of the MT data can be used to infer the deep resistivity structure of the crust to help investigate possible tectonic controls on the emplacement of mineral deposits along these linear trends.

\section{MAGNETOTELIURIC METHOD}

The magnetotelluric (MT) method is a passive surface geophysical technique, which uses the earth's natural electromagnetic fields to investigate the electrical resistivity structure of the subsurface. The resistivity of geologic units is largely dependent upon their fluid content, porosity, fracturing, temperature, and conductive mineral content (Keller, 1989). Saline fluids within the pore spaces and fracture openings can reduce resistivities in a resistive rock matrix. Also, resistivity can be lowered by the presence of conductive clay minerals, carbon, and metallic

mineralization. It is common for altered volcanic rocks to contain authigenic minerals that have resistivities ten times lower than those of the surrounding rocks (Nelson and Anderson, 1992). Increased temperatures cause higher ionic mobility and mineral activation energy, reducing rock resistivities significantly. Unaltered, unfractured igneous rocks are normally very resistive (typically 1,000 ohm-m or greater), whereas faults will show low resistivity (less than $100 \mathrm{ohm}-\mathrm{m}$ ) when they are comprised of rocks fractured enough to have hosted fluid transport and consequent mineralogical alteration (Eberhart-Phillips et al., 1995). Carbonate rocks are moderately to highly resistive (hundreds to thousands of ohm-m) dependent upon their fluid content, porosity, fracturing, and impurities. Marine shales, mudstones, and clay-rich alluvium are normally very conductive (a few ohm-m to tens of ohm-m). Unaltered, metamorphic rocks (nongraphitic) are moderately to highly resistive (hundreds to thousands of ohm-m). Tables of electrical resistivity for a 
variety of rocks, minerals and geological environments may be found in Keller (1987) and Palacky (1987).

The MT method allows us to probe the crust from depths of tens of meters to depths of tens of kilometers (Vozoff, 1991). Natural variations of the Earth's magnetic and electric field are measured and recorded at each MT station. Frequencies of $10,000 \mathrm{~Hz}$ to $1 \mathrm{~Hz}$ from worldwide lightning activity and $1 \mathrm{~Hz}$ to $0.0001 \mathrm{~Hz}$ from geomagnetic micro-pulsations are the main frequency bands used by the MT method. The natural electric and magnetic fields propagate vertically in the earth because the very large resistivity contrast between the air and the earth causes a vertical refraction of both fields transmitted into the earth (Vozoff, 1972).

The natural electric and magnetic fields are recorded in two orthogonal, horizontal directions. The vertical magnetic field ("tipper") is also recorded. The resulting time-series signals are used to derive earth tensor apparent resistivities and phases by first converting them to complex cross-spectra using FFT (fast-Fourier-transform) techniques. Least-squares, cross-spectral analysis (Bendat and Piersol, 1971) is used to solve for a tensor-transfer function that relates the observed electric fields to the magnetic fields under the assumption that the Earth consists of a two-input, two-output, linear system with the magnetic fields as input and the electric fields as output (Rodriguez and others, 1996). Prior to conversion to apparent resistivity and phase, the tensor is normally rotated into principal directions that usually correspond to the direction of maximum and minimum apparent resistivity. For a two-dimensional (2-D) Earth, the MT fields can be de-coupled into transverse electric (TE) and transverse magnetic (TM) modes; 2-D modeling is generally done to fit both modes. When the geology satisfies the 2-D assumption, the MT data for the TE mode is assumed to represent the situation when the electric field is along the geologic strike, and the data for the TM mode is assumed to represent the situation when the electric field is across strike. The MT method is well suited for studying complicated geological environments because the electric and magnetic relations are sensitive to vertical and horizontal variations in resistivity. The method is capable of establishing whether the electromagnetic fields are responding to subsurface terranes of effectively 1-, 2-, or 3-dimensions. An excellent introduction to the $\mathrm{MT}$ method and references for a more advanced understanding are contained in Dobrin and Savit (1988) and Vozoff (1991). 


\section{MAGNETOTELLURIC SURVEY}

Eighteen MT soundings were located along or near profile MT3-MT3" (Figure 1) with spacing that varied from 1.5 to 14.0 kilometers. The profile orientation is roughly perpendicular to the Battle Mountain-Eureka and Carlin trends. Stations 15, 15, and 16 were collected with our U.S. Geological Survey truck-mounted MT system (Stanley, 1978). Stations 30, 31, 35, and 99-106 were collected with our portable EMI MT-1 system (EMI, 1996). Horizontal electric fields were sensed using an L-shaped, three-electrode array with dipole lengths of $30 \mathrm{~m}$ except for stations 1-5, 15, and 16 where a dipole length of $37.5 \mathrm{~m}$ was used with our truck-mounted MT system. The orthogonal, horizontal magnetic fields in the direction of the electric-field measurement array were sensed using permalloycored induction coils (Stanley and Tinkler, 1983).

Frequencies sampled ranged from 300 to $0.004 \mathrm{~Hz}$ using single station recordings of both orthogonal horizontal components of the electric and magnetic fields, along with the vertical magnetic field at stations 30, 31, 35, and 99-106. Sampling this frequency range in previous areas of widely varying geology has allowed us to probe the crust from depths of hundreds of meters to depths of tens of kilometers.

The recorded time-series data were transformed to the frequency domain and Fourier analyzed to determine a twodimensional apparent resistivity and phase tensor at each site. The data were rotated to maximum and minimum apparent resistivity directions so that propagation modes for the signals were decoupled into TE and TM modes. Local reference sensors to help reduce bias in the impedance determinations due to instrument or environmental noise (Gamble and others, 1979a; Clarke and others, 1983) were used at stations 30, 31, and 35. Although true remote reference techniques were not used in our survey, we did sort cross-power files to select optimal signal-to-noise data sets (Appendix).

The effects of near-surface resistivity anomalies cause "static shifts" (Sternberg and others, 1988) in the data. Static shifts of this data set ranged from 0.0 to 1.3 of a log decade. Only stations 4, 16, 30, 31 and 105 had static shifts larger than one-third of a log decade, $1.3,0.7,0.5,0.4$, and 0.8 , respectively. The remainder of the stations had an average of 0.1 of a decade static shift. The larger static shifts should be accounted for in any subsequent modeling of the data. 


\section{MAGNETOTELLURIC DATA}

The following table shows eighteen magnetotelluric (MT) station locations (from southwest to northeast). Coordinates are referenced to the 1866 Clarke spheroid and North American 1927 Western United States datum. Longitude and latitude format below is decimal degrees. Elevation is in meters.

\begin{tabular}{|c|c|c|c|}
\hline Station & Longitude & Latitude & Elev (m) \\
\hline 16 & -117.03204 & 40.03355 & 1740 \\
\hline 106 & -116.99012 & 40.02843 & 1571 \\
\hline 15 & -116.92586 & 40.06778 & 1595 \\
\hline 1 & -116.84890 & 40.15161 & 1590 \\
\hline 2 & -116.75097 & 40.20433 & 1500 \\
\hline 105 & -116.77233 & 40.21090 & 1560 \\
\hline 5 & -116.66432 & 40.23551 & 1475 \\
\hline 3 & -116.58452 & 40.27633 & 1445 \\
\hline 4 & -116.57803 & 40.29375 & 1445 \\
\hline 99 & -116.59627 & 40.32478 & 1475 \\
\hline 100 & -116.44335 & 40.35050 & 1475 \\
\hline 101 & -116.32823 & 40.39182 & 1550 \\
\hline 102 & -116.25280 & 40.48377 & 1640 \\
\hline 103 & -116.10717 & 40.48735 & 1600 \\
\hline 104 & -115.98703 & 40.53218 & 1950 \\
\hline 30 & -116.81659 & 40.26598 & 1740 \\
\hline 31 & -116.80054 & 40.31094 & 1870 \\
\hline 35 & -116.64283 & 40.39057 & 1580 \\
\hline
\end{tabular}

The figures in the Appendix represent the raw field MT data for each station after the time series data was converted to the frequency domain and least-squares, cross-spectral analysis (Bendat and Piersol, 1971) was used to solve for a tensor-transfer function. This tensor transfer function was used to rotate the tensor into principal directions that correspond to the direction (rotation angle) of maximum and minimum apparent resistivity. Apparent resistivity and impedance phase were then calculated.

For each station, nine separate graph plots are given:

- Apparent Resistivity for two modes,

- Impedance Phase for two modes,

- Rotation Angle for the impedance tensor,

- Impedance Skew for the impedance tensor,

- Multiple Coherency for two modes of the electric field,

- Impedance Polar Plots (at 12 selected frequencies),

- Tipper Magnitude for the vertical magnetic field,

- Tipper strike for the vertical magnetic field, and

- HzHx (x symbol) and HzHy (o symbol) Coherency. 
Each of these graphs plots the above quantity versus frequency. The Apparent Resistivity, Impedance Phase, and Multiple Coherency plots use $x$ and o symbols to distinguish the two tensor modes. When inverting or modeling the data, these modes will be identified as TE and TM. The $x$ and 0 symbols on the $\mathrm{HzHx}$ and $\mathrm{HzHy}$ Coherency plot represent $\mathrm{HzHx}$ and HzHy coherency, respectively. Error bars are probable errors within one standard deviation of the sample variance (Gamble and others, 1979b).

Apparent resistivity is a measure of the magnitude of the electric field strength over the magnetic field strength for a given frequency. The impedance phase is proportional to the slope of the apparent resistivity curve on a log-log scale, but from a baseline at -45 degrees (Vozoff, 1991). A measure of the dimensionality for MT data is provided by the impedance skew of the impedance tensor (Vozoff, 1972). If the effective measured resistivity response to the geology beneath a MT station is truly $1-D$ or $2-D$, then the skew will be zero. Both instrument and environmental sources of noise contribute to non-zero skew values, but are typically small (about 0.1) for relatively low noise level recordings. Higher skews (above 0.2) are an indication of either 3-D resistivity responses to the geology or higher levels of noise. Man-made electrical noise, such as power lines, power generators, moving vehicles and trains can have a negative effect on MT data quality. All these local disturbances produce an incoherent noise mainly affecting higher frequencies, usually above $1 \mathrm{~Hz}$. Other man-made electrical noise, such as direct current electric trains and active cathodic protection of pipelines produce coherent electromagnetic signals mainly affecting frequencies below $1 \mathrm{~Hz}$.

In the survey area, noise from a number of small power lines and small moving vehicles was negligible at distances of $0.4 \mathrm{~km}$ and greater. Power line levels were measured at each site and were typically less than $20 \%$ of the maximum recordable signals. Noise from larger power lines, power generators, pipelines, railroads, and steam-driven trains, mostly near mining operations, was negligible at least $5 \mathrm{~km}$ from them. Recordings were not made when noise from moving vehicles affected the magnetic signals. Local lightning, wind, and rainstorms can also degrade data quality, but these were avoided by not recording during active thunderstorm periods. Wind noise was minimized by burying the magnetic induction coils. 
Predicted values of the electric field can be computed from the measured values of the magnetic field (Vozoff, 1991). The coherence of the predicted electric field with the measured electric field is a measure of the signal-to-noise ratio provided in the multiple coherency plot. Values are normalized between 0 and 1 , where values at 0.5 signify signal levels equal to noise levels. For this data set, coherencies were generally at an acceptable level, except at times in the "dead band" (0.1 to $1 \mathrm{~Hz})$ and at times in the lower frequencies $(0.004$ to 0.1$)$. The lower frequency ionospheric signals are related to sunspot activity whose levels typically follow an 11-year cycle. The sunspot activity was near the lowest level of the cycle during the 1994, 1996, and 1997 surveys, and was near the highest level in the 2000 survey.

The figures in the Appendix represent the raw field MT data at each station, which includes some data scatter and poor signal-to-noise ratios. Our only effort at removing noisy data points was to visually inspect and select the best signal-to-noise field data to combine into the final data plots.

The impedance polar plots provide a measure of the MT data dimensionality (Reddy and others, 1977). For 1-D resistivity structures, the principal impedance polar diagrams are circles. For 2-D or 3-D resistivity structures, the principal impedance polar diagrams (dashed lines) elongate either parallel or perpendicular to strike direction. Over resistors, the principal impedance polar diagrams elongate perpendicular to strike direction and over conductors, the principal impedance polar diagrams elongate parallel to strike direction. Also, for 2-D resistivity structures, the additional impedance polar diagrams (solid lines) attain the shape of a symmetric clover leaf. For 3-D resistivity structures, the additional impedance polar diagrams elongate in one direction and their amplitudes are comparable to those of principal impedances. Sites whose polar plots indicated 3D character in the lower frequencies were MT stations 3, 4, 100, and 101 (Figure 1).

The tipper can be solved for when the vertical component of the magnetic field is measured. The tipper magnitude is a measure of the tipping of the magnetic field out of the horizontal plane (Vozoff, 1991). The magnitude is zero for the $1-\mathrm{D}$ case and typically increases between 0.1 to 0.5 , and rarely as great as 1 , as it responds to vertical and subvertical structures. The tipper strike is typically used to help resolve the 90-degree ambiguity in the impedance rotation 
angle. The vertical component of the magnetic field was measured at MT stations 30, 31, 35, and 99-106. The tipper magnitude of these stations was typically 0.1 to 0.4 over the lower frequencies indicating vertical structure at depth. The $\mathrm{HzHx}$ and HzHy coherency is a measure of the signal-to-noise ratio of the vertical magnetic field with respect to each of the orthogonal horizontal magnetic field directions. Values are normalized between 0 and 1 , where values at 0.5 signify signal levels equal to noise levels. These three-component magnetic field coherencies provide a check on the signal-tonoise ratio of the measured values in the tipper magnitude and tipper strike plots.

\section{REFERENCES CITED}

Arehart, G.B., Foland, K.A., Naeser, C.W., and Kesler, S.E., 1993, 40Ar/39Ar, K/Ar, and fission track geochronology of sediment-hosted disseminated gold deposits at post-Betze, Carlin Trend, northeastern Nevada: Economic Geology, vol. 88 , no. 3, p. $622-646$.

Bendat, J.S., and Piersol, A.G., 1971, Random Data: Analysis and Measurement Procedures: New York, Wiley Interscience, $407 \mathrm{p}$.

Clarke, J., Gamble, T.D., Goubau, W.M., Koch, R.H., and Miracky, R.F., 1983, Remote-reference magnetotellurics: Equipment and procedures: Geophysical Prospecting, vol. 31 , p. 149-170.

Dobrin, M.D., and Savit, C.H., 1988, Introduction to Geophysical Prospecting (4th ed.): New York, McGraw-Hill, $867 \mathrm{p}$.

Eberhart-Phillips, D., Stanley, W. D., Rodriguez, B. D. and Lutter, W. J., 1995, Surface seismic and electrical methods to detect fluids related to faulting: Journal of Geophysical Research, vol. 100, no. B7, p. 12,919-12,936.

EMI, 1996, MT-1 magnetotelluric system operation manual, Version 3.2: ElectroMagnetic Instruments, Inc., Richmond, California, $220 \mathrm{p}$.

Gamble, T.D., Goubau, W.M. and Clarke, J., 1979a, Magnetotellurics with a remote magnetic reference: Geophysics, v. 44, no. 1, p. 53-68. 
Gamble, T.D., Goubau, W.M. and Clarke, J., 1979b, Error analysis for remote reference magnetotellurics: Geophysics, v. 44, no. 5, p. 959-968.

Ilchik, R.P. and Barton, M.D., 1997, An amagmatic origin of Carlin-type gold deposits: Economic Geology, vol. 92, no. 3 , p. 269-288.

Keller, G.V., 1987, Rock and mineral properties, in Electromagnetic Methods in Applied Geophysics Theory: M.N. Nabighian, Ed., Society of Exploration Geophysicists, Tulsa, Oklahoma, v. 1, p. 13-51.

Keller, G.V., 1989, Electrical properties, in Carmichael, R.S., Ed., Practical handbook of physical properties of rocks and minerals: CRC Press, Boca Raton, Florida, p. $359-427$.

Nelson, P.H. and Anderson, L.A., 1992, Physical properties of ash flow tuff from Yucca Mountain, Nevada: Journal of Geophysical Research, vol. 97, no. B5, p. 6823-6841.

Palacky, G.J., 1987, Resistivity characteristics of geologic targets, in Electromagnetic Methods in Applied Geophysics Theory: M.N. Nabighian, Ed., Society of Exploration Geophysicists, Tulsa, Oklahoma, vol. 1, p. 53-129.

Radtke, A.S., 1985, Geology of the Carlin gold deposit, Nevada: U.S. Geological Survey Professional Paper 1267, $124 \mathrm{p}$.

Reddy, I.K., Rankin, D., and Phillips, R.J., 1977, Threedimensional modeliing in magnetotelluric and magnetic variational sounding: Geophysics Journal of the Royal Astronomical society, vol. 51, p. 313-325.

Rodriguez, B.D., Stanley, W.D., and Williams, J.M., 1996, Axial structures within the Reelfoot rift delineated with magnetotelluric surveys: U.S. Geological Survey Professional Paper 1538-K, $30 \mathrm{p}$.

Shawe, D.R., 1991, Structurally controlled gold trends imply large gold resources in Nevada, in Geology and ore deposits of the Great Basin, Symposium Proceedings: Raines, G.L., Lisle, R.E., Schafe, R.W., Wilkinson, W.H., Eds., Geological Society of Nevada, Reno, vol. 1, p. 199212 . 
Sillitoe, R.H. and Bonham, H.F., 1990, Sediment-hosted gold deposits; distal products of magmatic-hydrothermal systems: Geology, vol. 18, no. 2, p. 157-161.

Stanley, W.D., 1978, New magnetotelluric field processing system: U.S. Geological Survey Professional Paper 1100 , $174 \mathrm{p}$.

Stanley, W.D., and Tinkler, R.D., 1983, A practical, low-noise coil system for magnetotellurics: U.S. Geological Survey Open-File Report 83-85, $18 \mathrm{p}$.

Sternberg, B.K., Washburne, J.C., and Pellerin, L., 1988, Correction for the static shift in magnetotellurics using transient electromagnetic soundings: Geophysics, vol. 53, p. 1459-1468.

Struhsacker, E.M., Jones, E., and Green, S.M., 1996, Roadside geology and precious-metal mineralization along the I-80 corridor, Reno to Elko, Nevada, in Struhsacker, E.M. and Green, S.M., eds., Geology and ore deposits of the American Cordillera - Field Trip Guidebook Compendium: Geological Society of Nevada, Reno, Nevada, p. 3.

Tosdal, R.M., 1998, Contributions to the gold metallogeny of northern Nevada: U.S. Geological Survey Open-File Report $98-338,290 \mathrm{p}$.

Vozoff, K., 1972, The magnetotelluric method in the exploration of sedimentary basins: Geophysics, vol. 37, p. 98-141.

Vozoff, K., 1991, The magnetotelluric method, in Electromagnetic methods in applied geophysics: M.N. Nabighian, Ed., Society of Exploration Geophysicists, Tulsa, Oklahoma, vol. 2, part B, p. 641-711. 


\section{APPENDIX}

\section{MAGNETOTELLURIC DATA PLOTS}

For stations 30, 31, 35, and 99-106, there are nine separate graph plots:

- Apparent Resistivity for two modes,

- Impedance Phase for two modes,

- Rotation Angle for the impedance tensor,

- Impedance Skew for the impedance tensor,

- Multiple Coherency for two modes of the electric field,

- Impedance Polar Plots (at 12 selected frequencies),

- Tipper Magnitude for the vertical magnetic field,

- Tipper strike for the vertical magnetic field, and

- HzHx (x symbol) and HzHy (o symbol) Coherency.

Stations 1-5, 15, and 16, only have the first six graph plots above, since the vertical magnetic field data (Tipper, $\mathrm{Hz}$ ) was not acquired. Refer to the "Magnetotelluric Data" section in this report for an explanation of these plots. 
Station 16

APPARENT RESISTIVITY

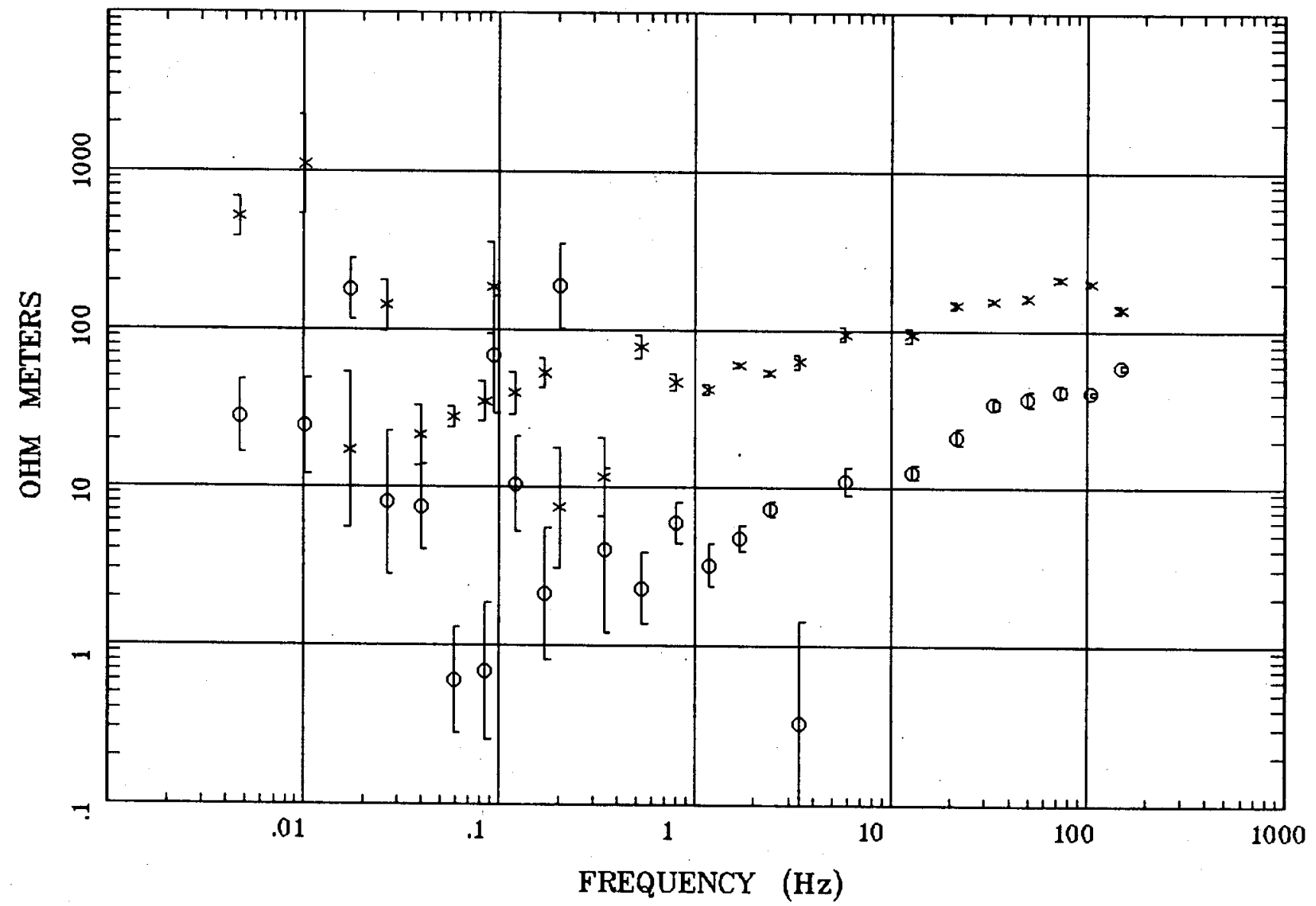

Client:

Remote:

Acquired:

Survey Co:
Rotation:

Filename: CT16C

Channels: Ch1 Ch2 Ch3 Ch4 Ch5 Ch6 Ch7

Plotted: 15:52 Dec 11, 2000

< EMI - ElectroMagnetic Instruments 


\section{Station 16}

\section{IMPEDANCE PHASE}

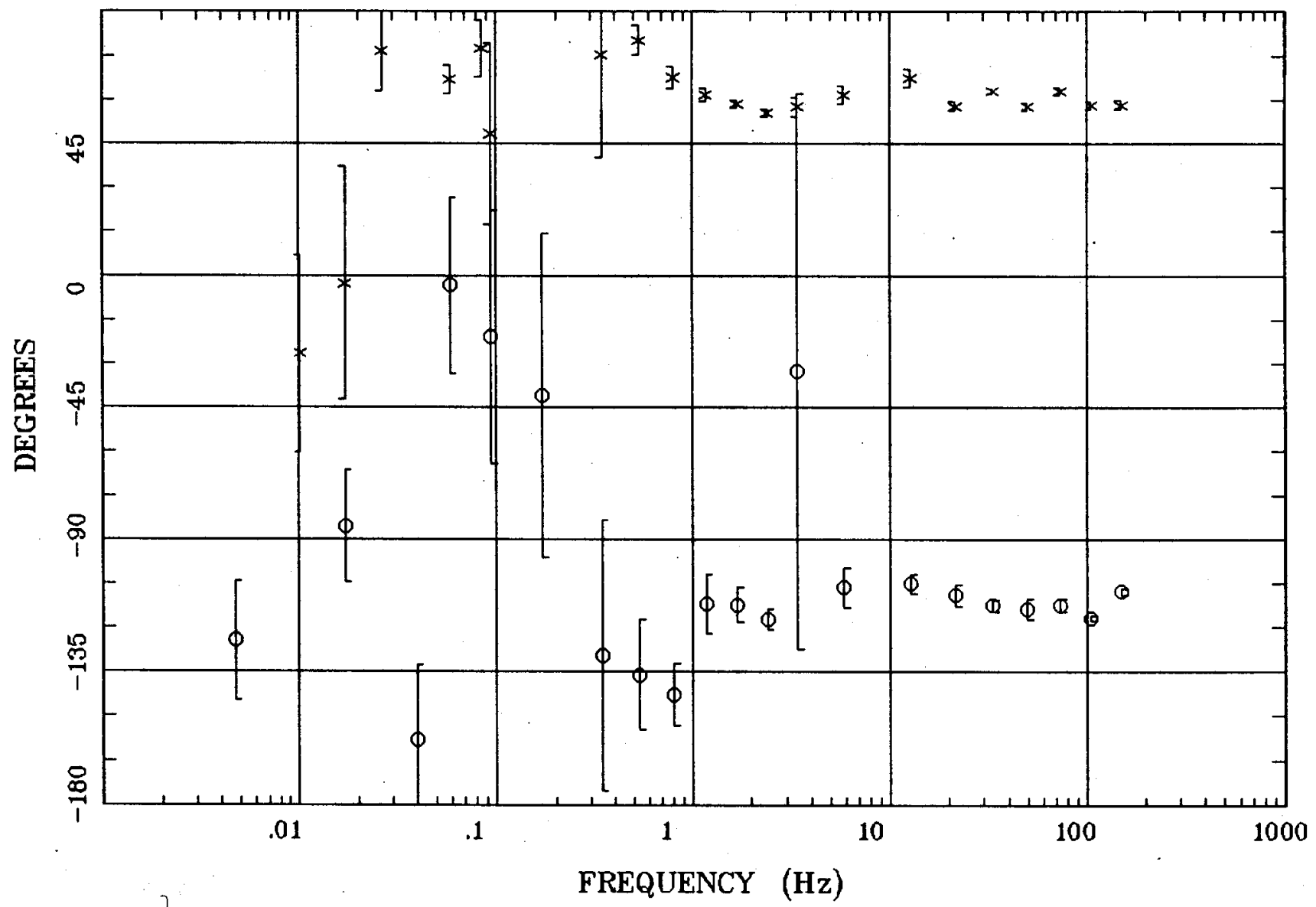

Client:

Remote:

Acquired:

Survey Co:
Rotation:

Filename: CT16C

Channels: Ch1 Ch2 Ch3 Ch4 Ch5 Ch6 Ch7 Plotted: 15:52 Dec 11, 2000

$<$ EMI - ElectroMagnetic Instruments 


\section{ROTATION ANGLE}

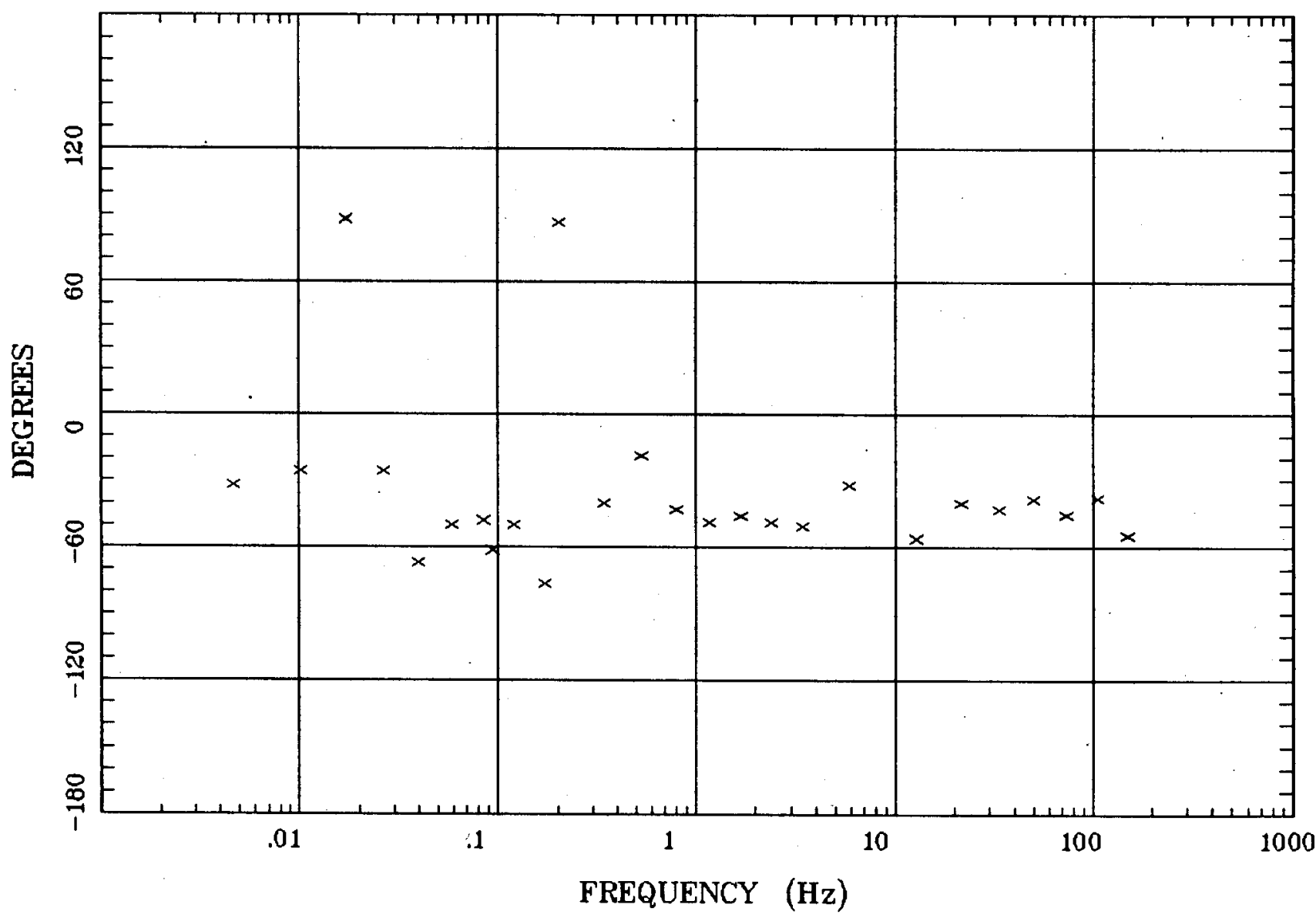

Client:

Remote:

Acquired:

Survey Co:
Rotation:

Filename: CT16C

Channels: Ch1 Ch2 Ch3 Ch4 Ch5 Ch6 Ch7

Plotted: 15:52 Dec 11. 2000

$<$ EMI - ElectroMagnetic Instruments > 
Station 16

\section{IMPEDANCE SKEW}

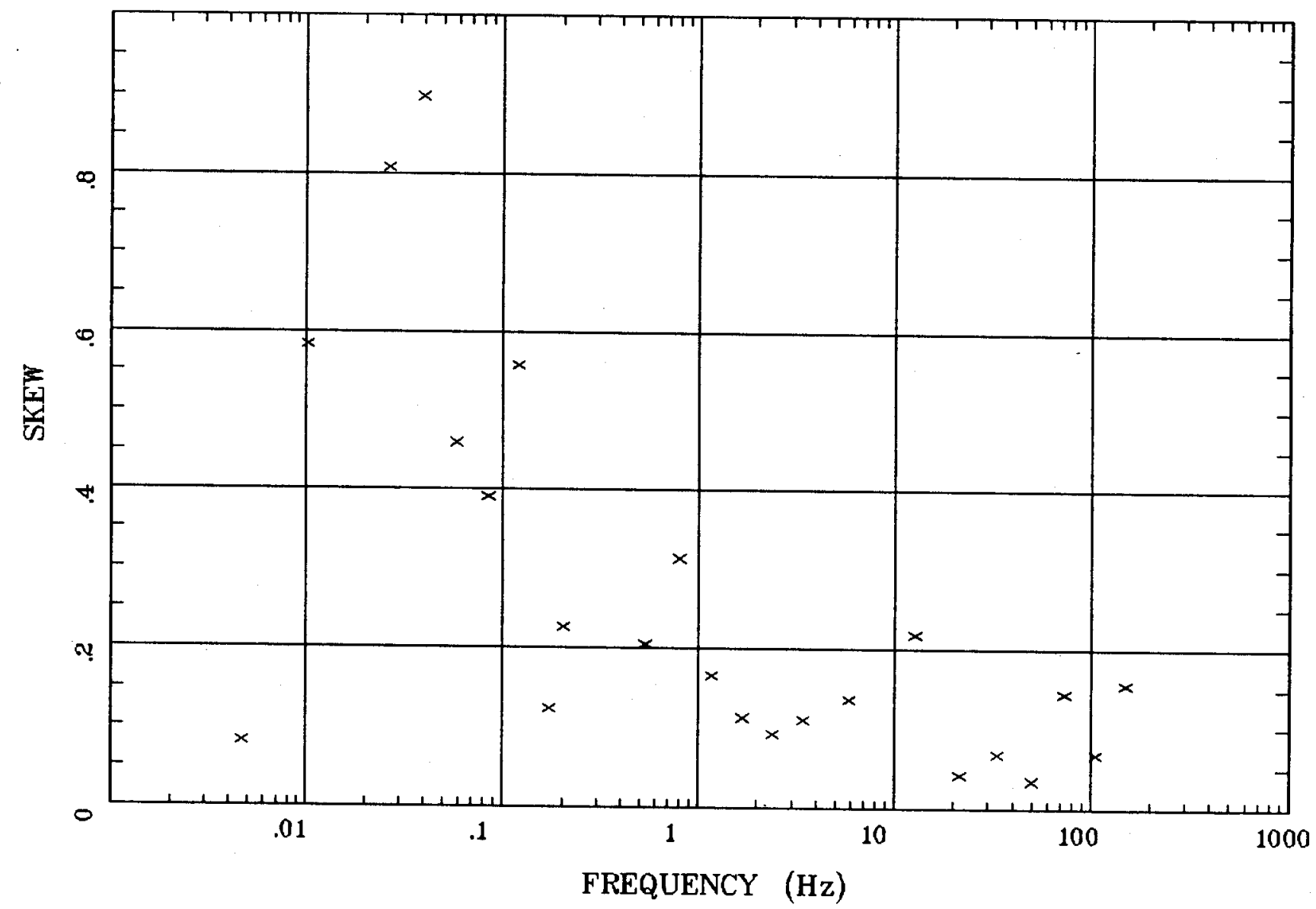

Client:

Remote:

Acquired:

Survey ca:

\section{Rotation:}

Filename: CT16C

Channels: Ch1 Ch2 Ch3 Ch4 Ch5 Ch6 Ch7

Plotted: 15:52 Dec 11, 2000

< EMI - ElectroMagnetic Instruments > 
Station 16

E MULT Coh.

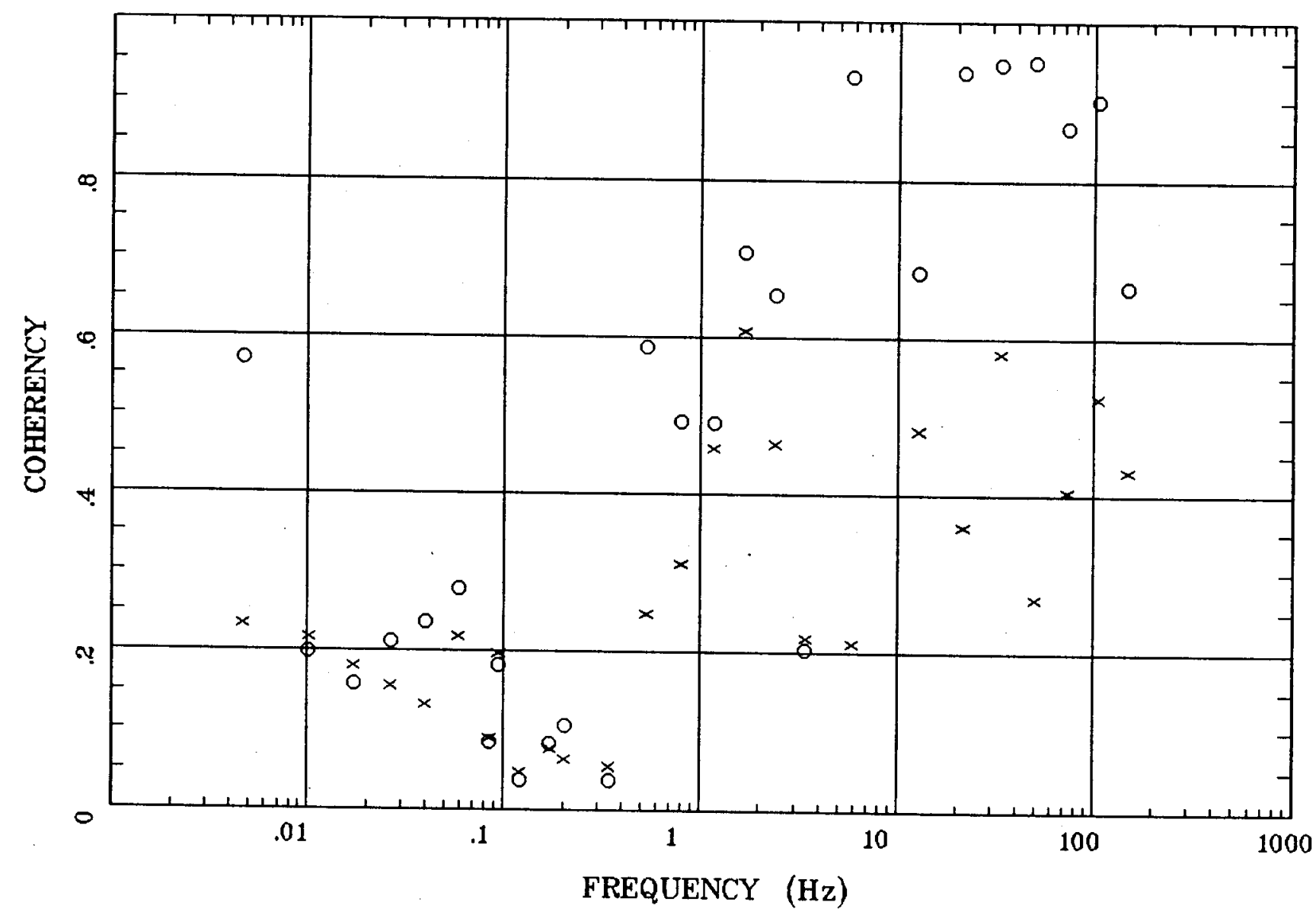

Client:

Remote:

Acquired:

Survey $\mathrm{Co}$ :
Rotation:

Fileneme: CT16C

Channels: Ch1 Ch2 Ch3 Ch4 Ch5 Ch6 Ch7 Plotted: 15:52 Dec 11, 2000

< EMI - ElectroMagnetic Instruments 
Station 16

POLAR PLOTS

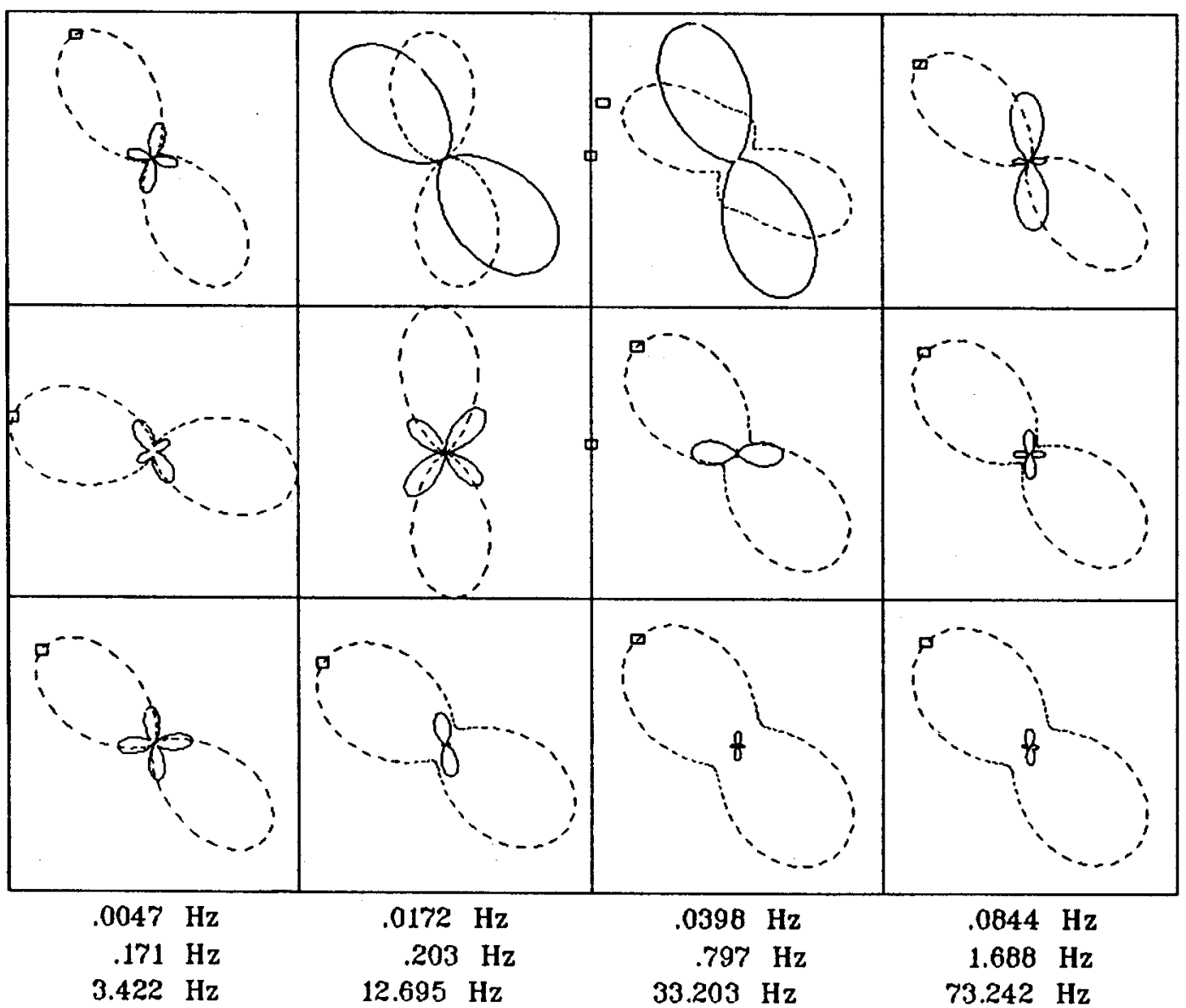

Rotation:

Client:

Remote:

Acquired:

Survey Co:
Filename: CT16C

Channels: Ch1 Ch2 Ch3 Ch4 Ch5 Ch6 Ch7

Plotted: 15:52 Dec 11, 2000

$<$ EMI - ElectroMagnetic Instruments 


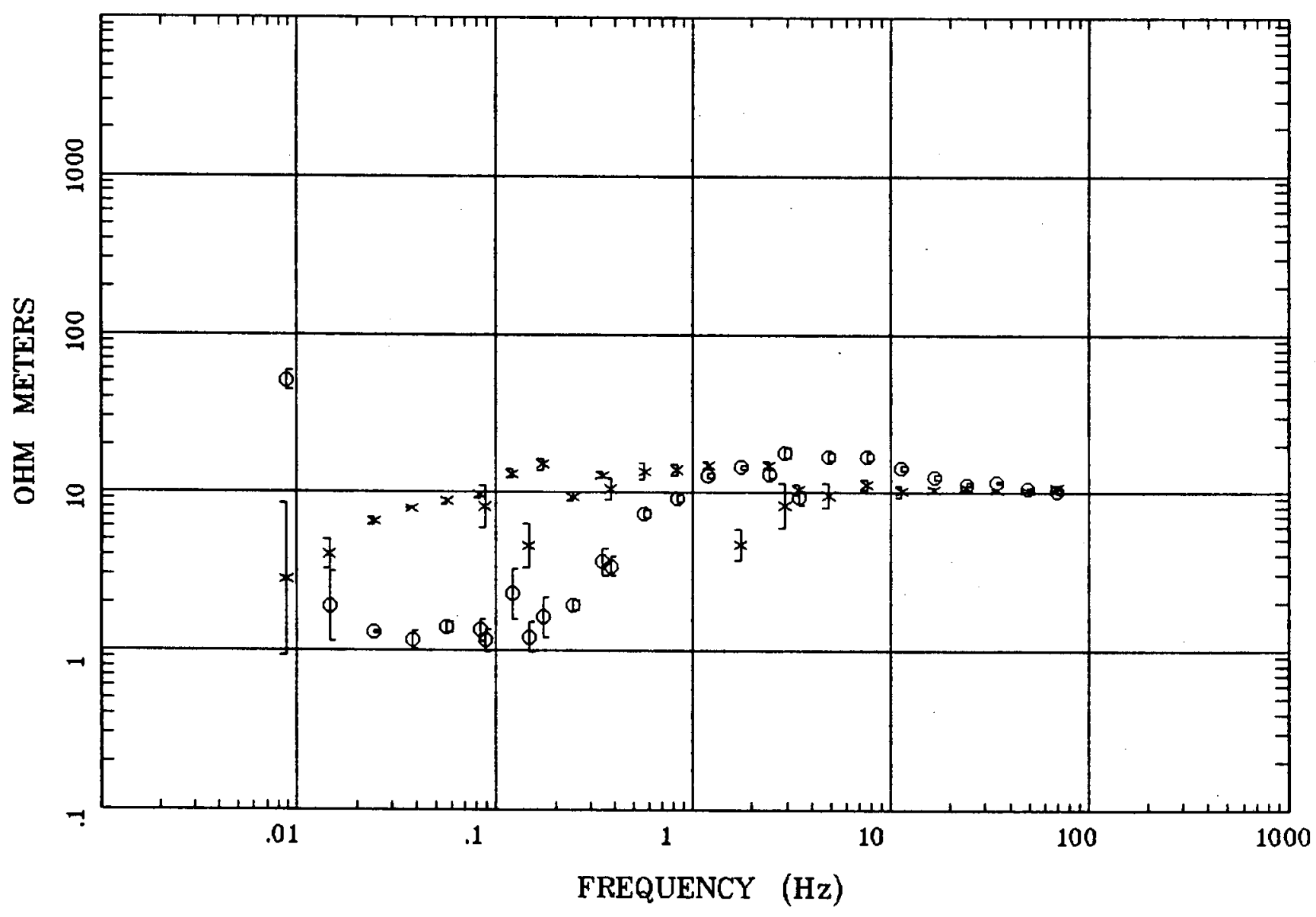

Client:

Remote: none

Acquired: 13:5 Aug 04, 2000

Survey Co:USGS
Rotation:

Filename: hr106c.avg

Channels: Ch1 Ch2 Ch3 Ch4 Ch5 Ch3 Ch4

Plotted: 15:23 Jan 24, 2001

<EMI - ElectroMagnetic Instruments > 


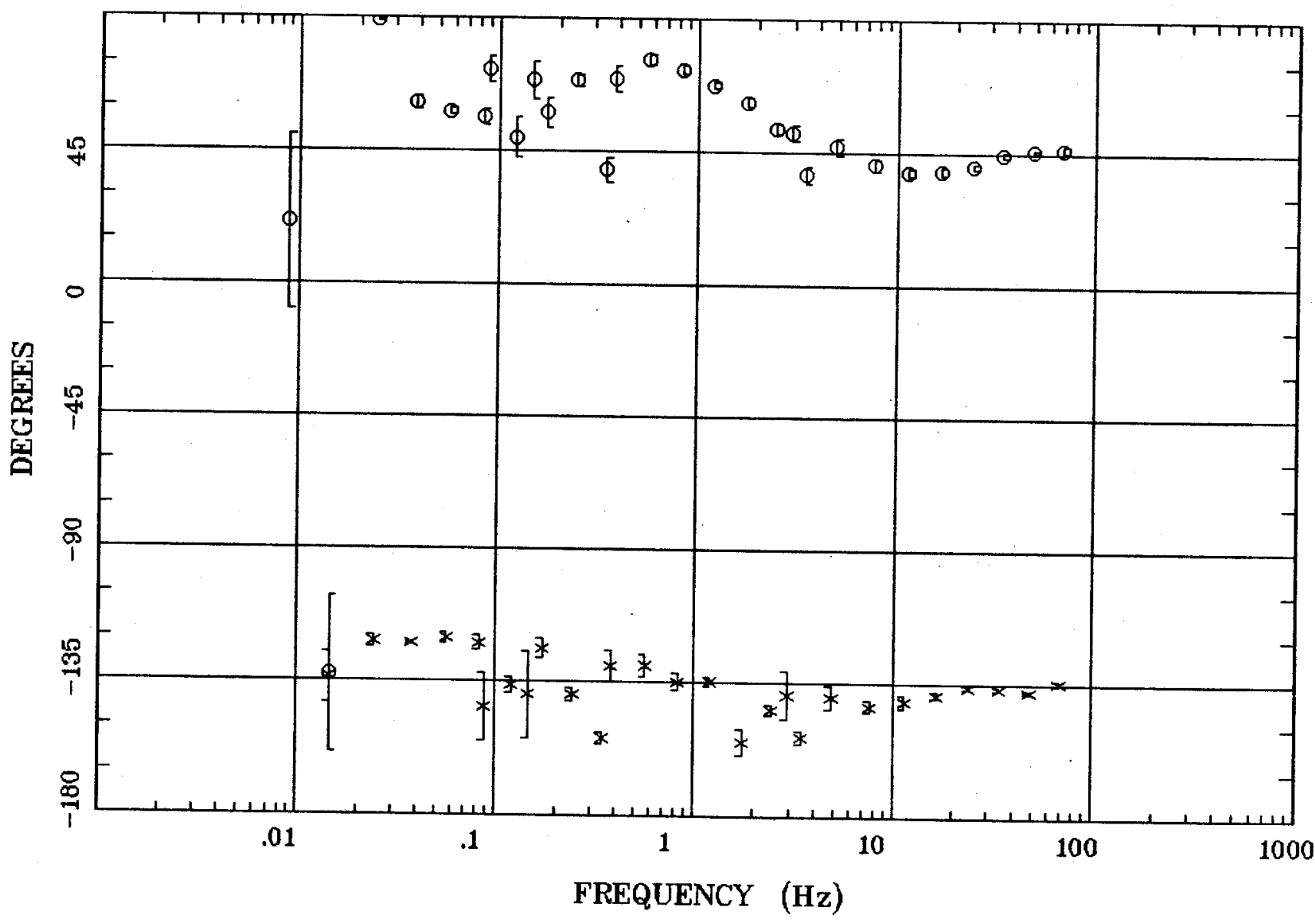

Client:

Remote: none

Acquired: 13:5 Aug 04, 2000

Survey Co:USGS
Rotation:

Filename: hr106c.avg

Channels: Ch1 Ch2 Ch3 Ch4 ch5 Ch3 Ch4

Plotted: 15:23 Jan 24, 2001

< EMI - ElectroMagnetic Instruments 


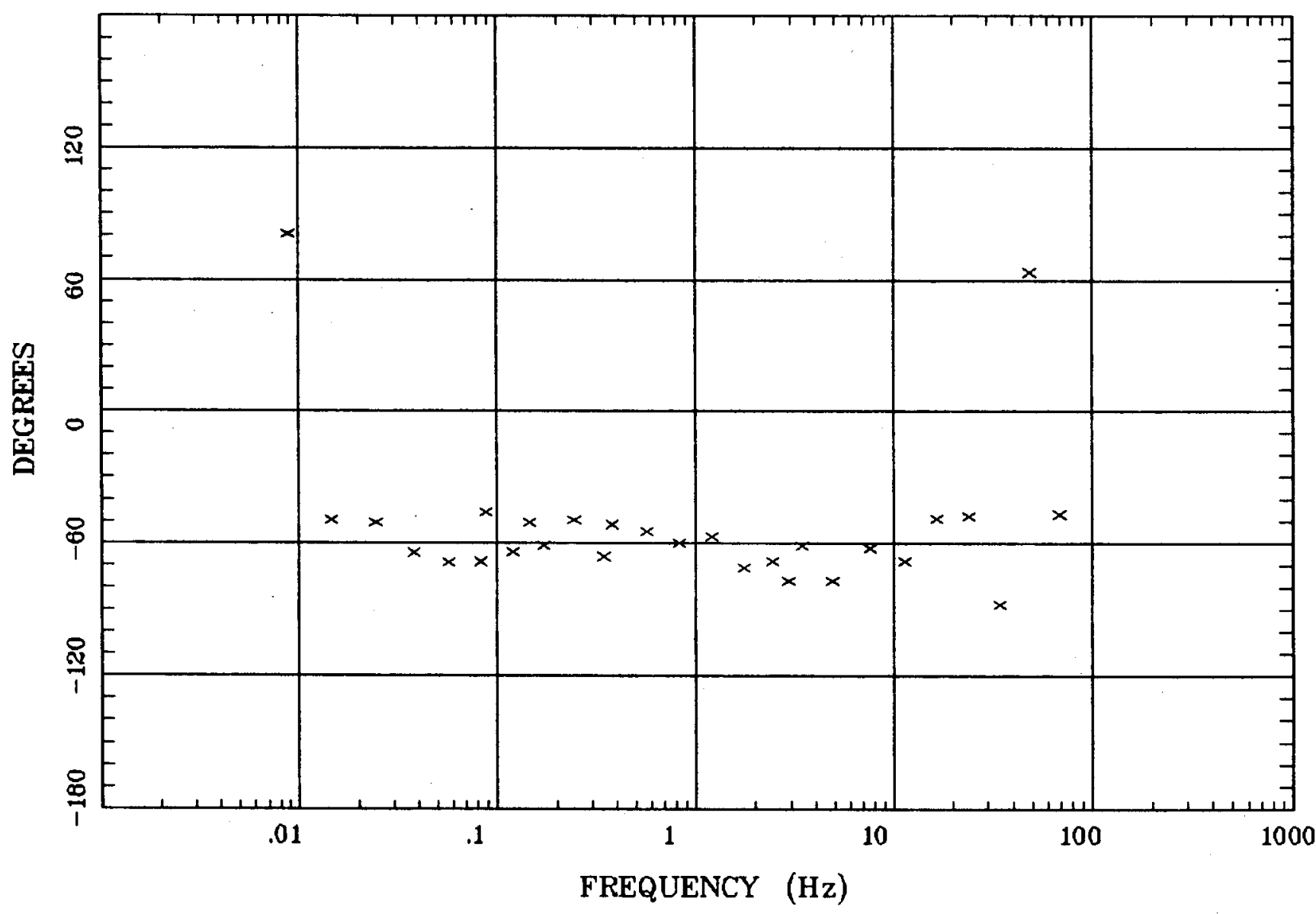

Client:

Remote: none

Acquired: 13:5 Aug 04, 2000

Survey Co:USGS
Rotation:

Filename: hr106c.avg

Channels: Ch1 Ch2 Ch3 Ch4 Ch5 Ch3 Ch4 Plotted: 15:23 Jan 24, 2001

< EMI - ElectroMagnetic Instruments > 
Station 106

IMPEDANCE SKEW

Crescent Valley, NV $100 \mathrm{~K}$

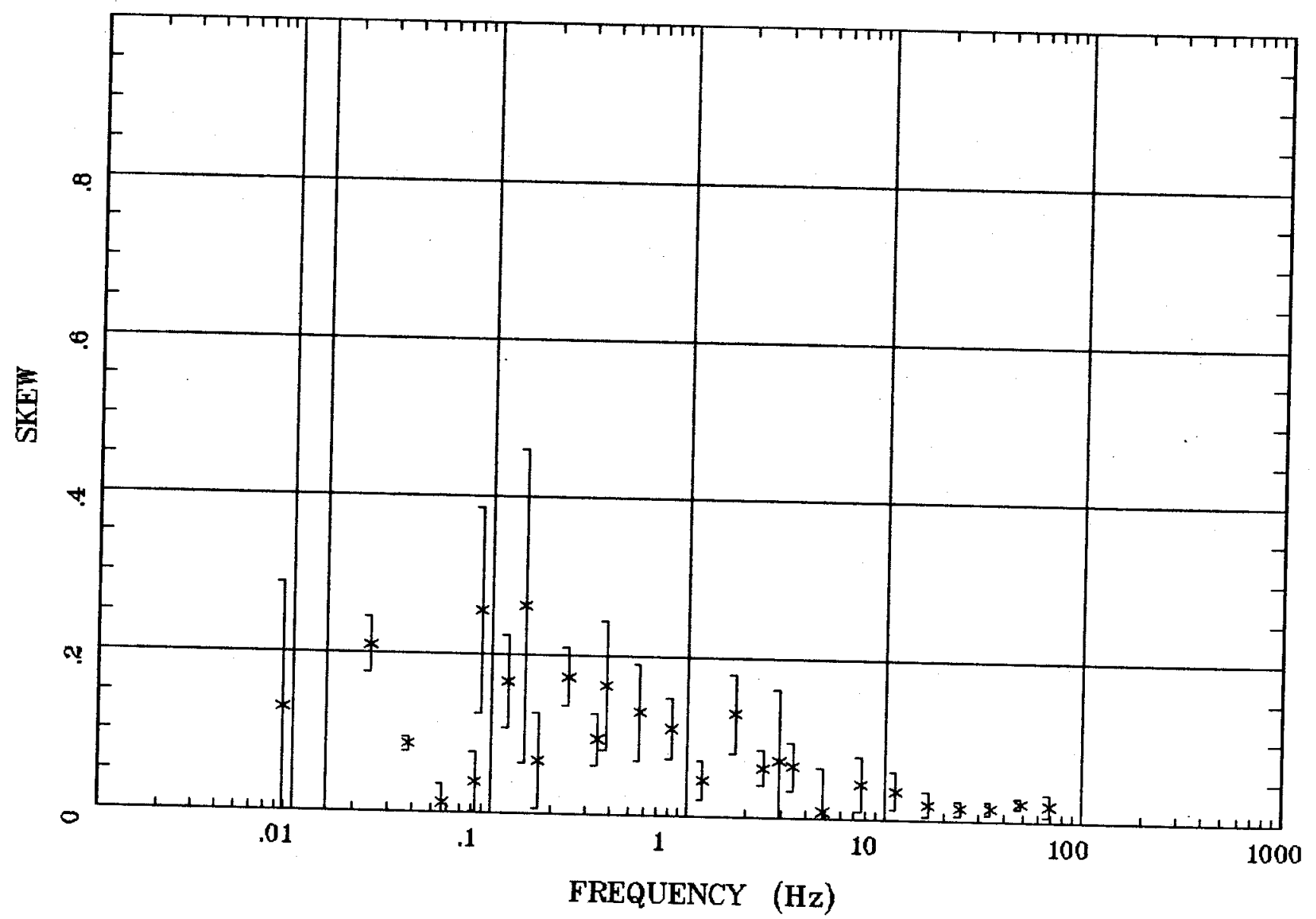

Client:

Rotation:

Remote: none

Filename: hr106c.avg

Channels: Ch1 Ch2 Ch3 Ch4 Ch5 Ch3 Ch4

Acquired: 13:5 Aug 04, 2000 Survey Co:USGS

Plotted: 15:23 Jan 24, 2001

< EMI - ElectroMagnetic Instruments > 


\section{Station 106}

E MULT Coh.

Crescent Valley, NV 100K

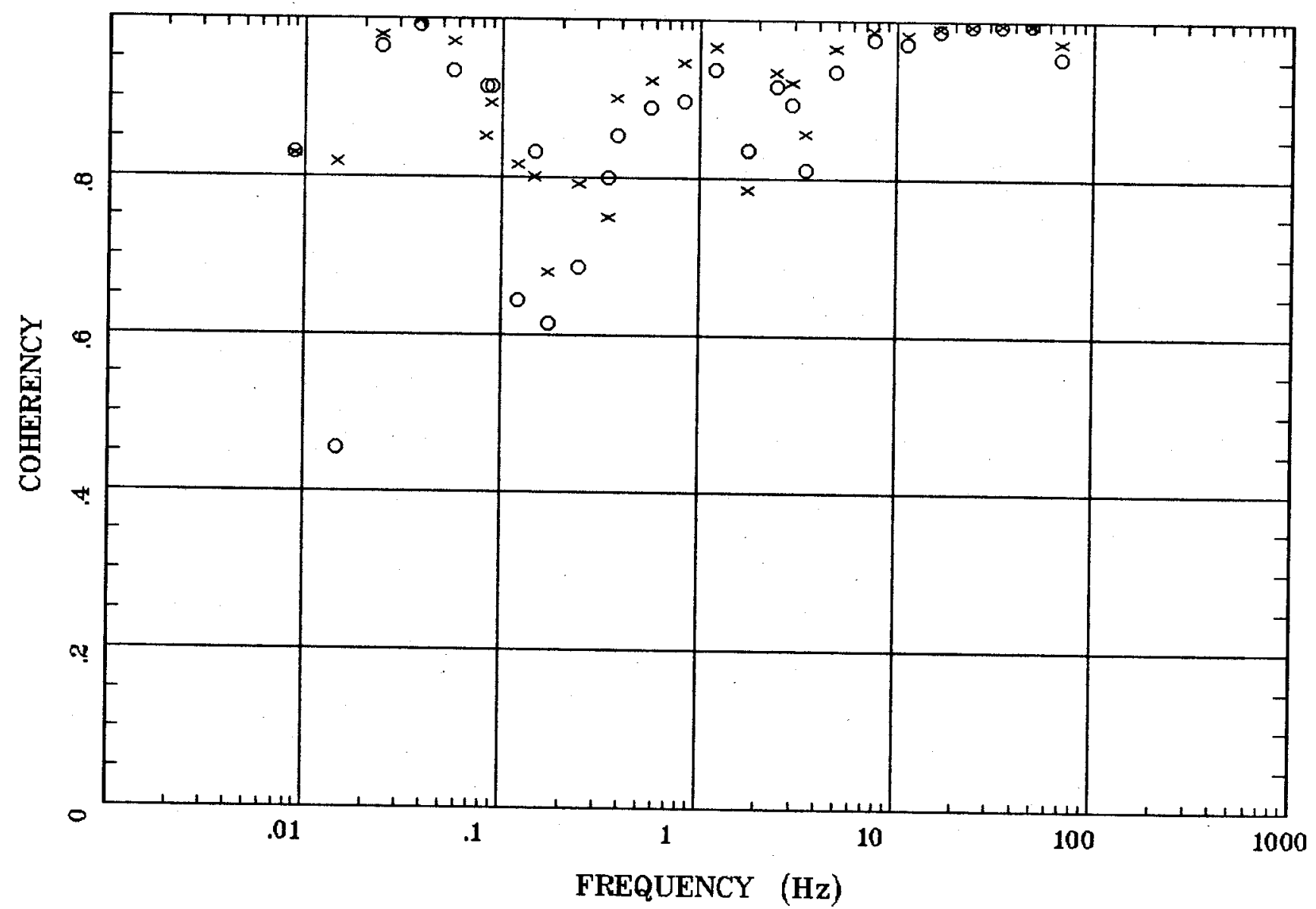

Client:

Remate: none

Acquired: $13: 5$ Aug 04, 2000

Survey Co:USGS
Rotation:

Filename: hr106c.avg

Channels: Ch1 Ch2 Ch3 Ch4 Ch5 Ch3 Ch4

Plotted: 15:23 Jan 24, 2001

< EMI - ElectroMagnetic Instruments > 


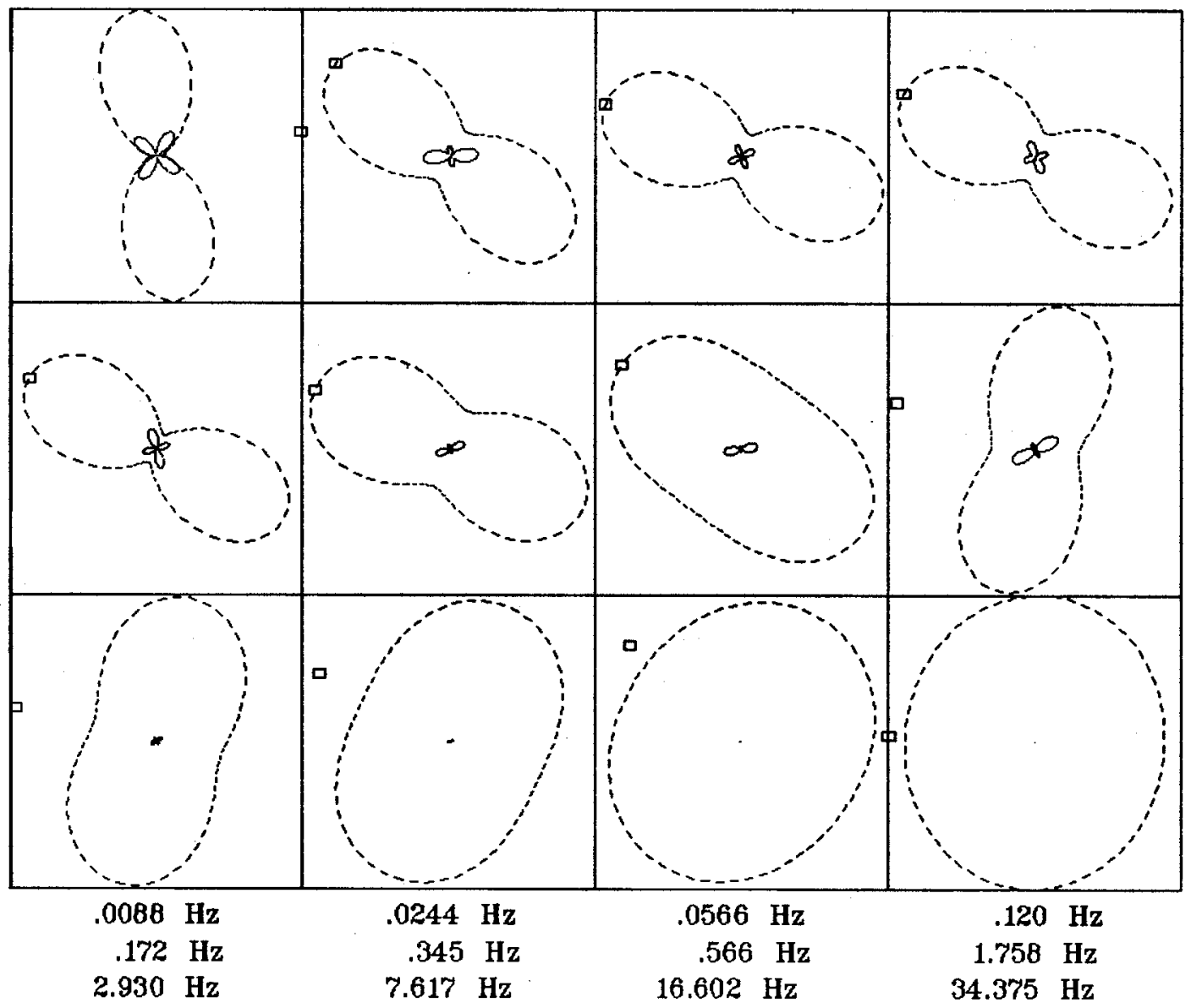

Client:

Remote: none

Acquired: 13:5 Aug 04, 2000 Survey Co:USGS

\section{Rotation:}

Filename: hr106c.avg

Channels: Ch1 Ch2 Ch3 Ch4 Ch5 Ch3 Ch4 Plotted: 15:23 Jan 24, 2001

< EMI - ElectroMagnetic Instruments > 


\section{Station 106}

\section{TIPPER MAGNITUDE}

Crescent Valley, NV 100K

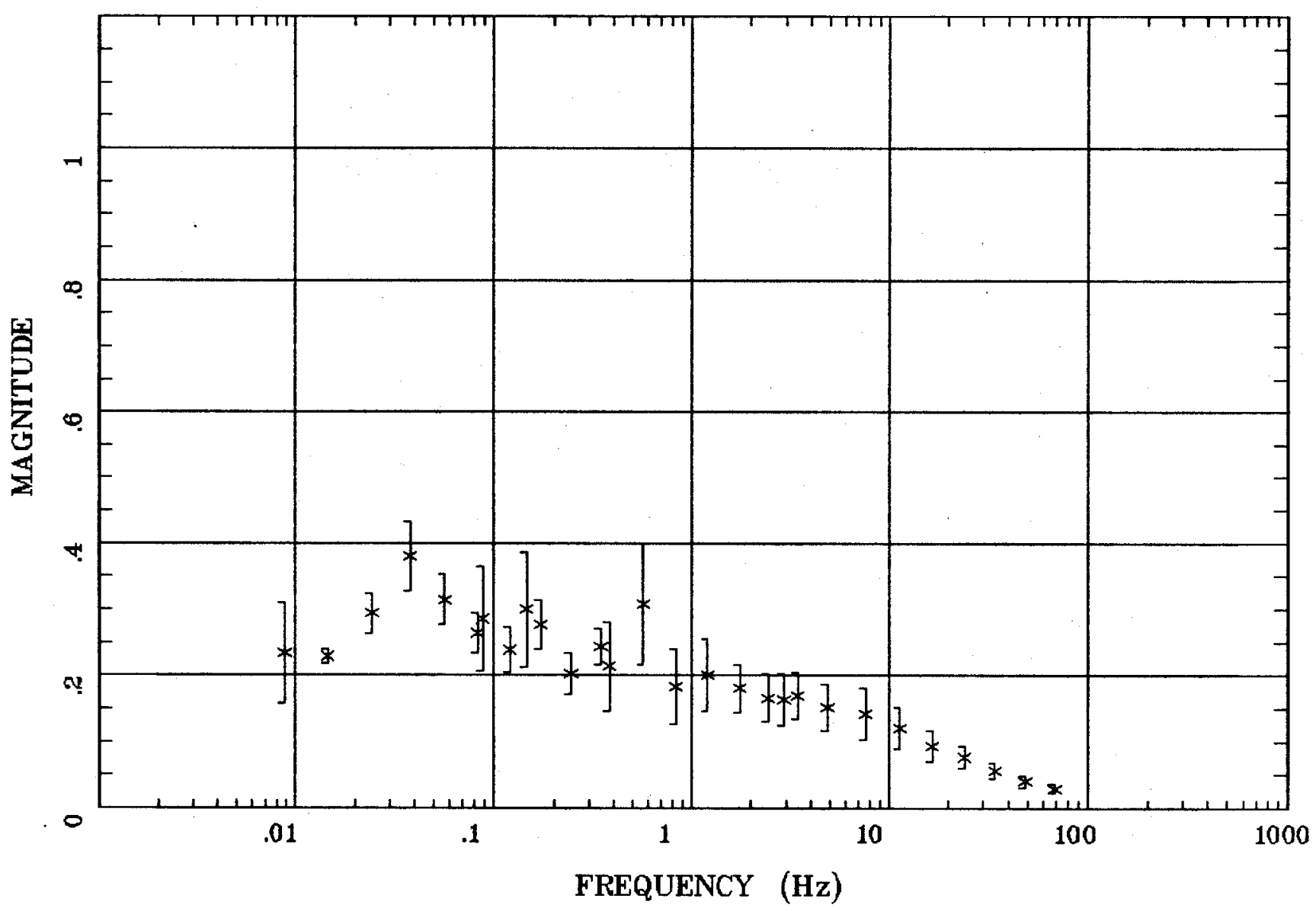

Client:

Rotation:

Filename: hr106c.avg

Remote: none

Acquired: 13:5 Aug 04, 2000

Channels: Ch1 Ch2 Ch3 Ch4 Ch5 Ch3 Ch4

Plotted: 15:23 Jan 24, 2001

Survey Co:USGS

< EMI - ElectroMagnetic Instruments > 
TIPPER STRIKE

Crescent Valley, NV 100K

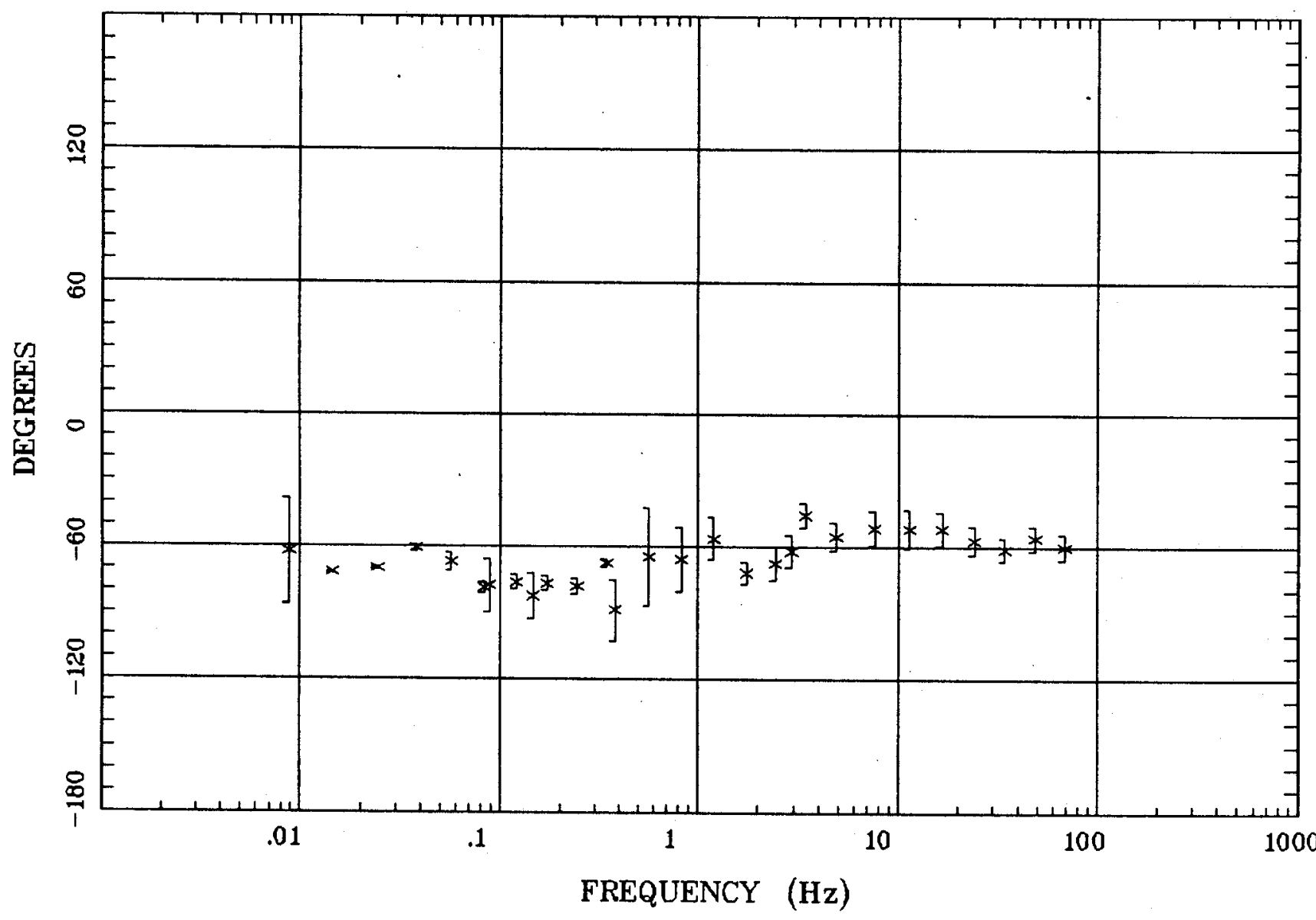

Client:

Remote: none

Acquired: 13:5 Aug 04, 2000

Survey Co:USGS
Rotation:

Filename: hr106c.avg

Channels: Ch1 Ch2 Ch3 Ch4 Ch5 Ch3 Ch4

Plotted: 15:23 Jan 24, 2001

< EMI - ElectroMagnetic Instruments 


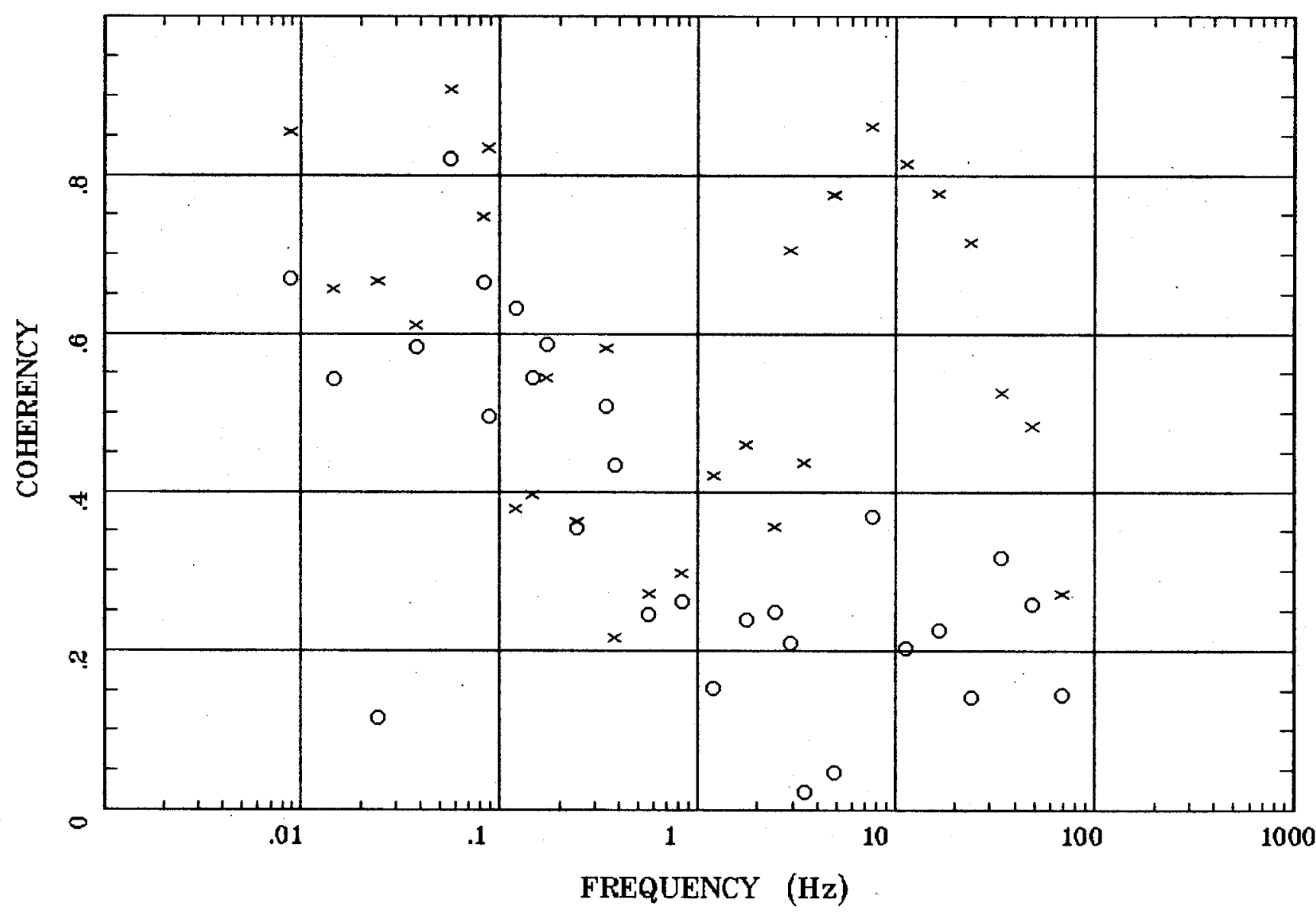

Client:

Remote: none

Acquired: 13:5 Aug 04, 2000 Survey Co:USGS
Rotation:

Filename: hr106c.avg

Channels: Ch1 Ch2 Ch3 Ch4 Ch5 ch3 Ch4

Plotted: 15:23 Jan 24, 2001

< EMI - ElectroMagnetic Instruments 


\section{Station 15}

APPARENT RESISTIVITY

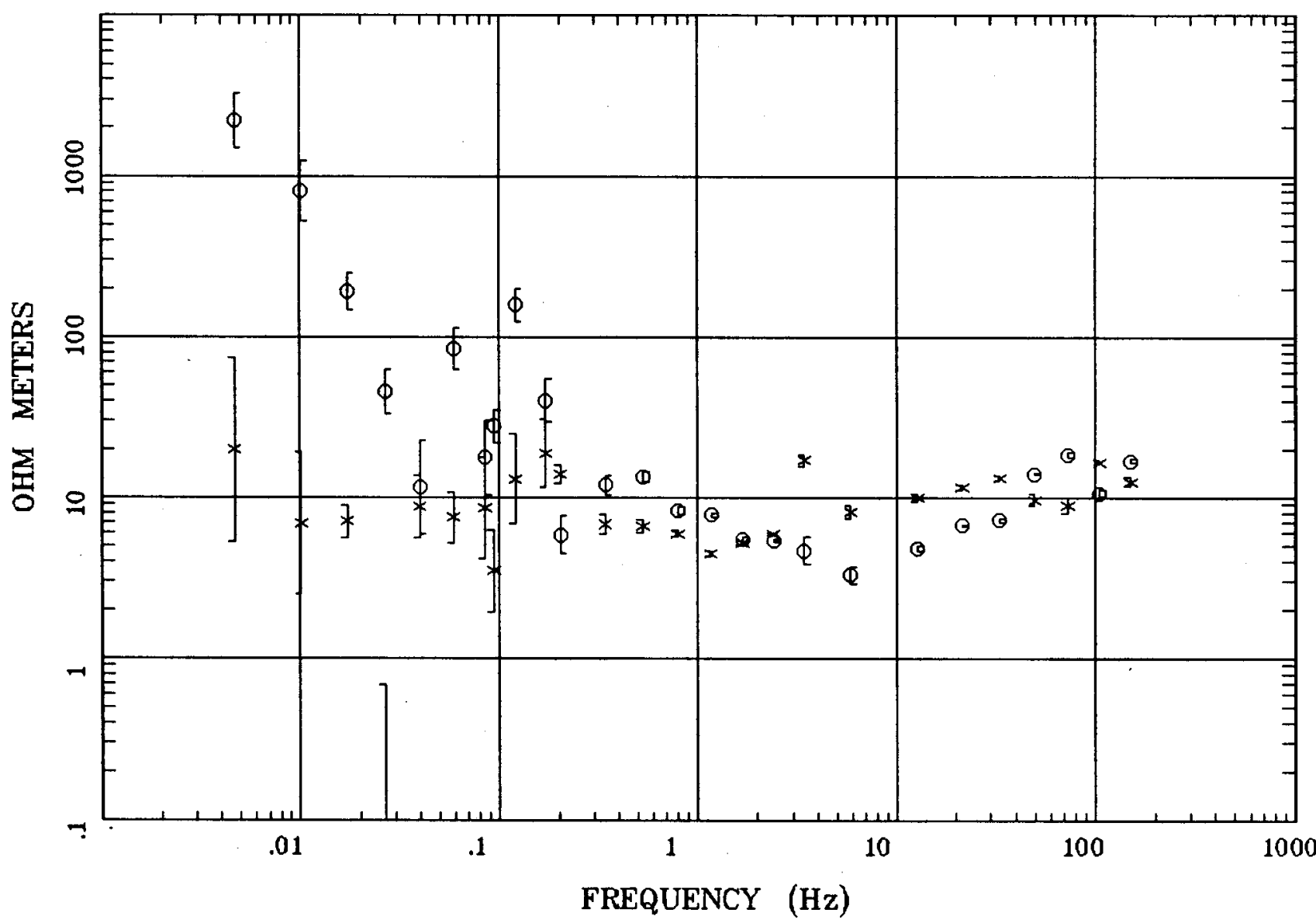

Client:

Remote:

Acquired:

Survey Co:
Rotation:

Filename: CT15F

Channels: Ch1 Ch2 Ch3 Ch4 Ch5 Ch6 Ch7

Plotted: 15:49 Dec 11, 2000

< EMI - ElectroMagnetic Instruments 


\section{Station 15}

\section{IMPEDANCE PHASE}

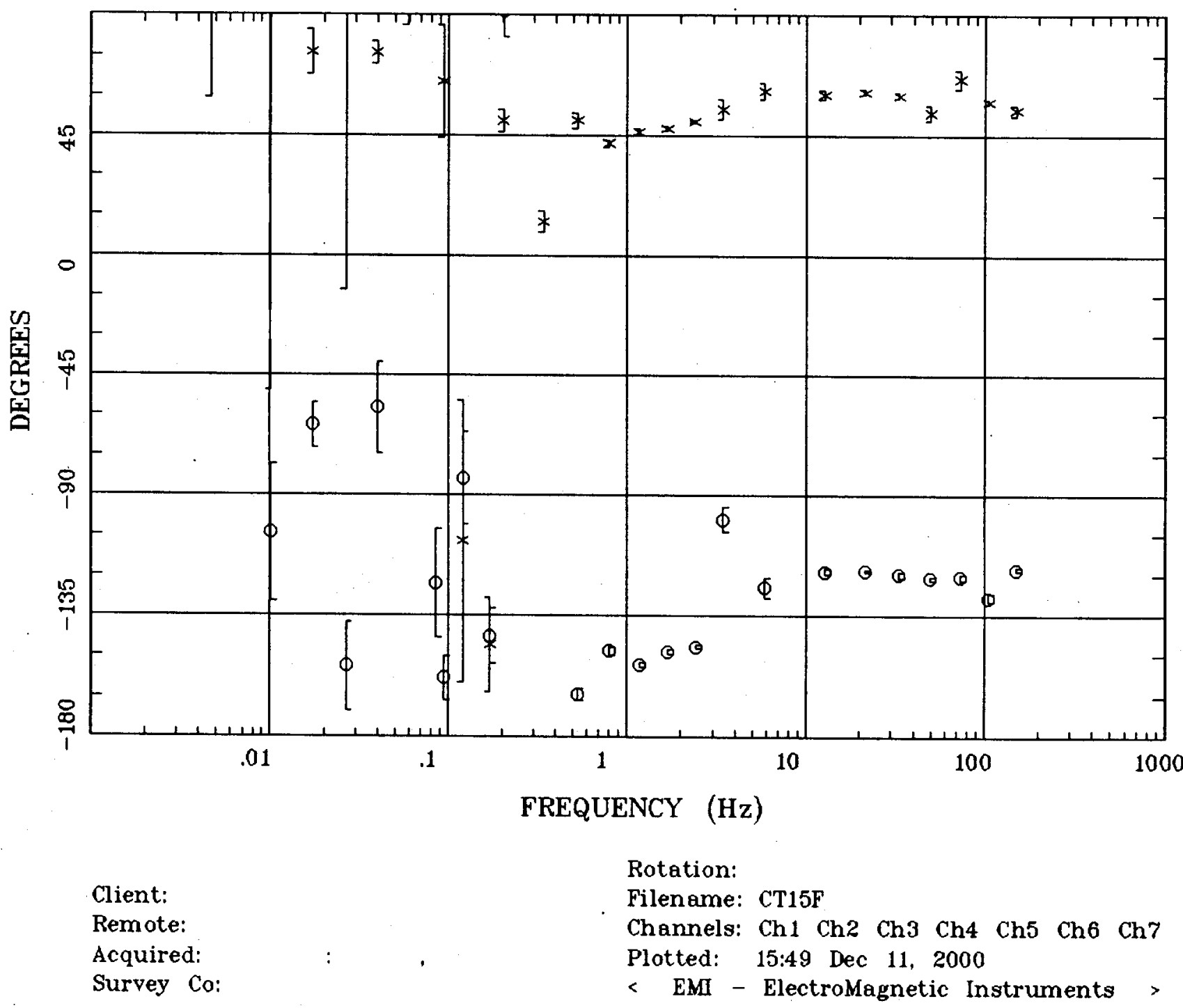




\section{ROTATION ANGLE}

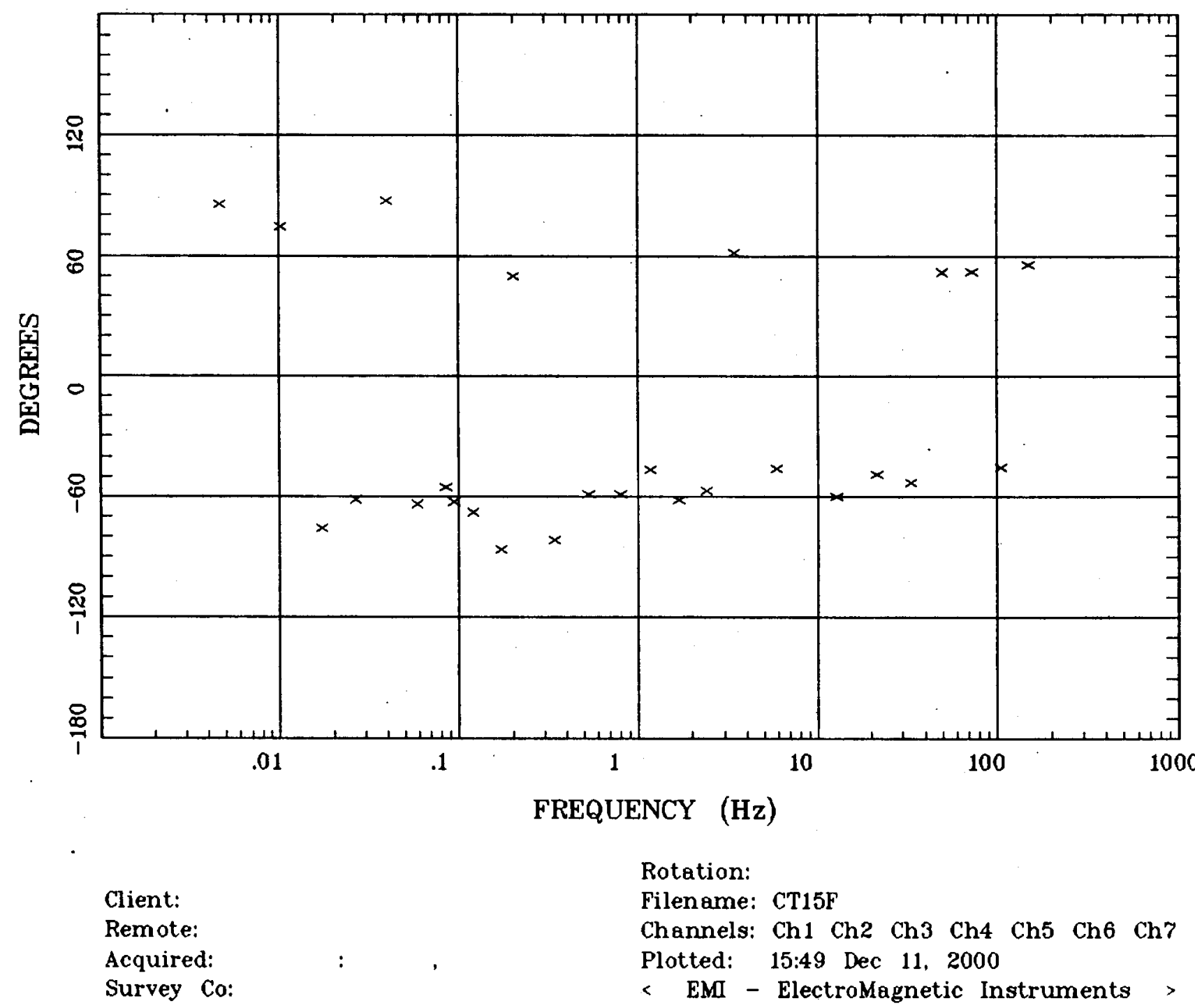




\section{Station 15}

\section{IMPEDANCE SKEW}

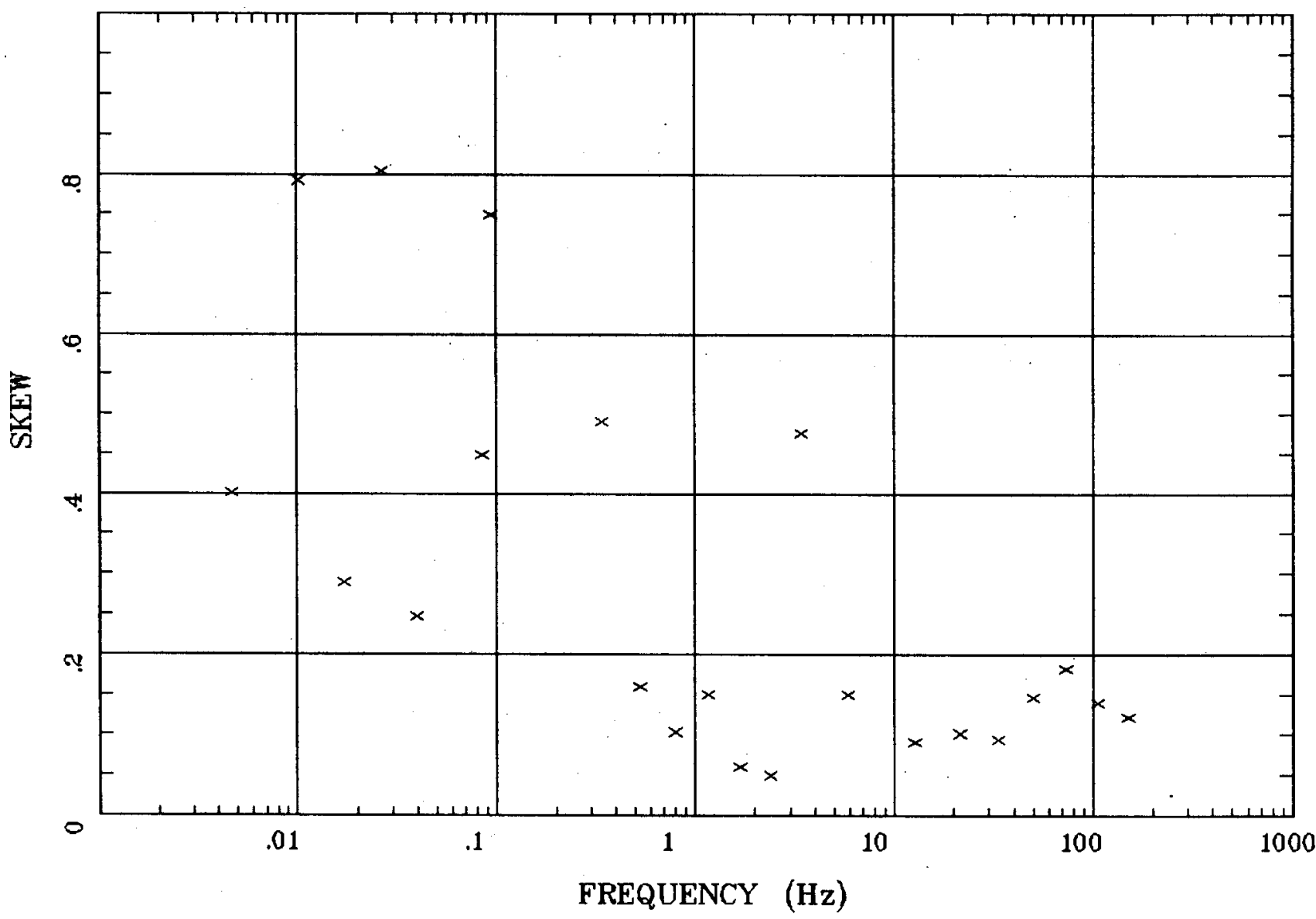

Client:

Rotation:

Remote:

Acquired:

Survey Co:
Filename: CT15F

Channels: Ch1 Ch2 Ch3 Ch4 Ch5 Ch6 Ch7

Plotted: 15:49 Dec 11, 2000

< EMI - ElectroMagnetic Instruments 


\section{E MULT Coh.}

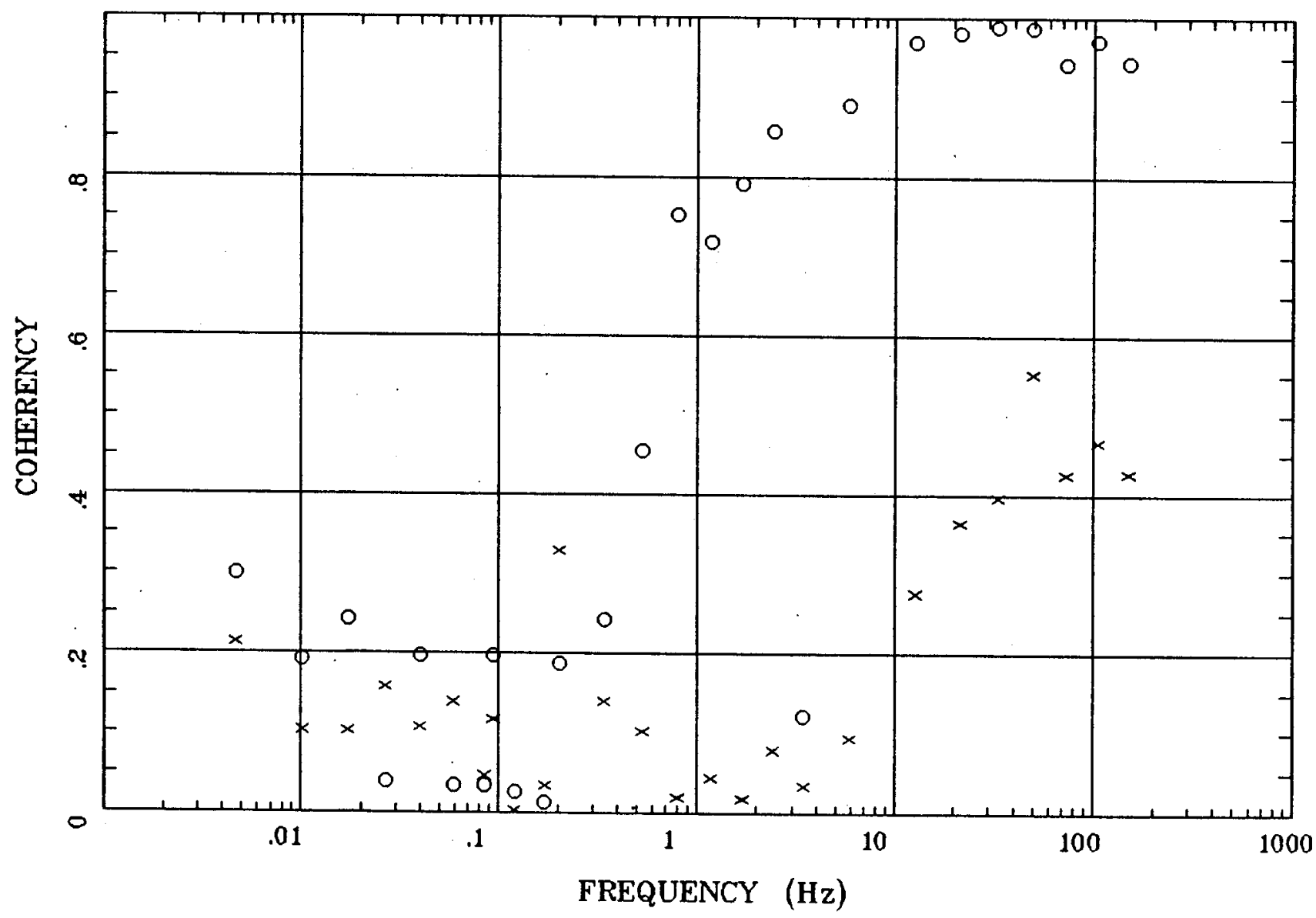

Client:

Remote:

Acquired:

Survey Co:
Rotation:

Filename: CT15F

Channels: Ch1 Ch2 Ch3 Ch4 Ch5 Ch6 Ch7

Plotted: 15:49 Dec 11. 2000

$<$ EMI - ElectroMagnetic Instruments > 


\section{POLAR PLOTS}

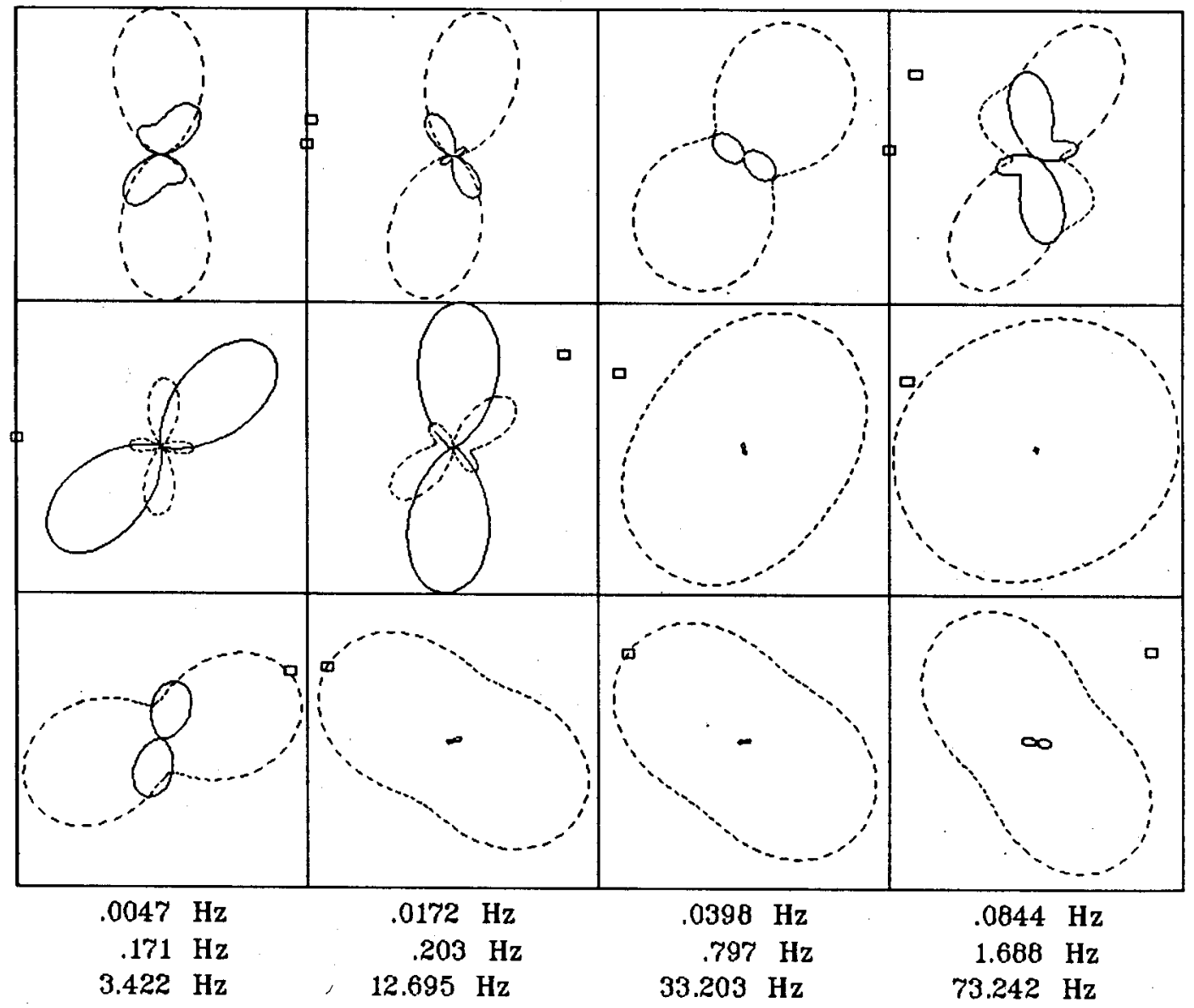

Client:

Remote:

Acquired:

Survey Co:
Rotation:

Filename: CT15F

Channels: Ch1 Ch2 Ch3 Ch4 Ch5 Ch6 Ch7

Plotted: 15:49 Dec 11, 2000

$<$ EMI - ElectroMagnetic Instruments 


\section{APPARENT RESISTIVITY}

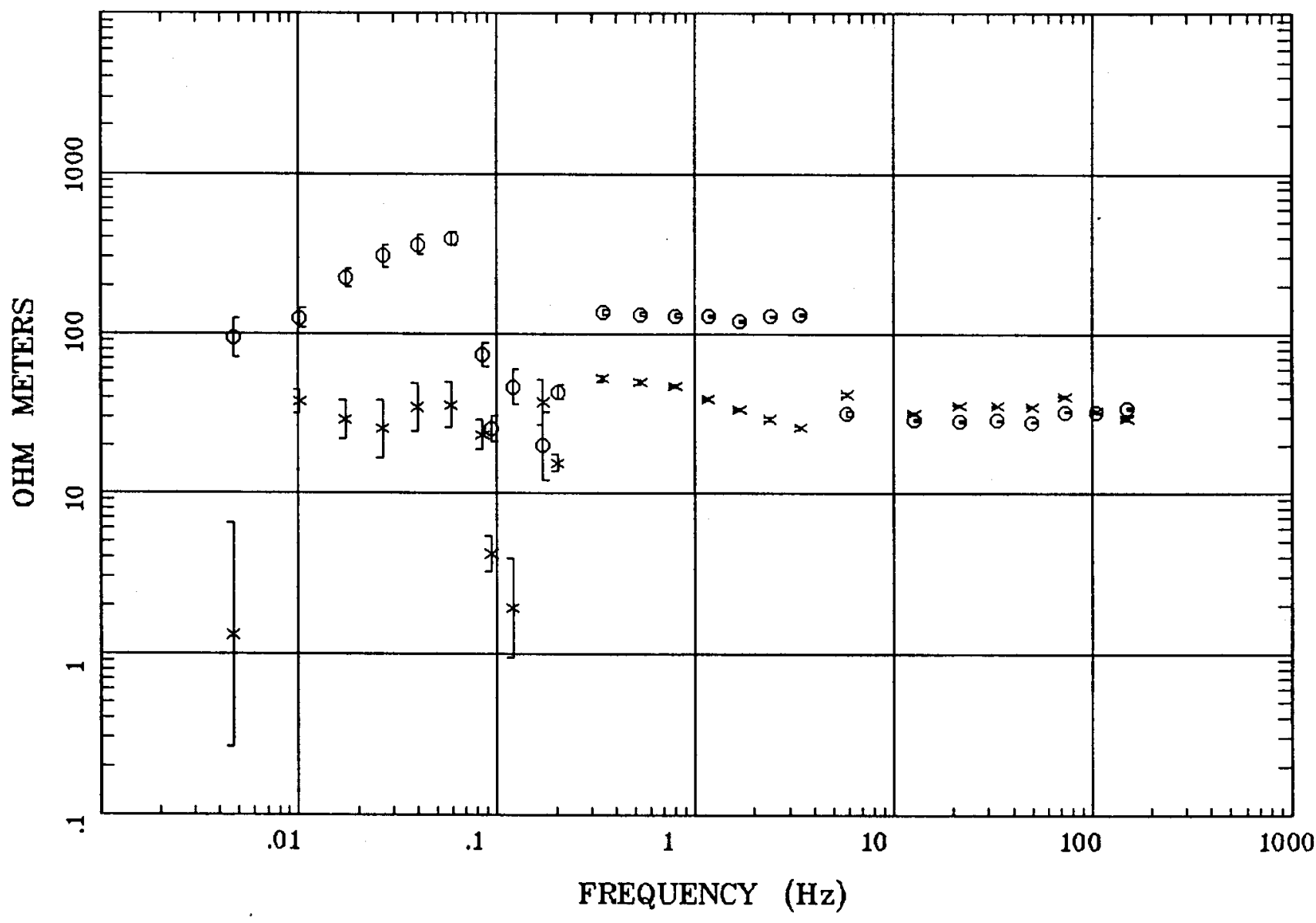

Client:

\section{Remote:}

Acquired: Survey Co:
Rotation:

Filename: NNR01

Channels: Ch1 Ch2 Ch3 Ch4 Ch5 Ch6 Ch7 Plotted: 15:29 Dec 11, 2000

< EMI - ElectroMagnetic Instruments 


\section{IMPEDANCE PHASE}

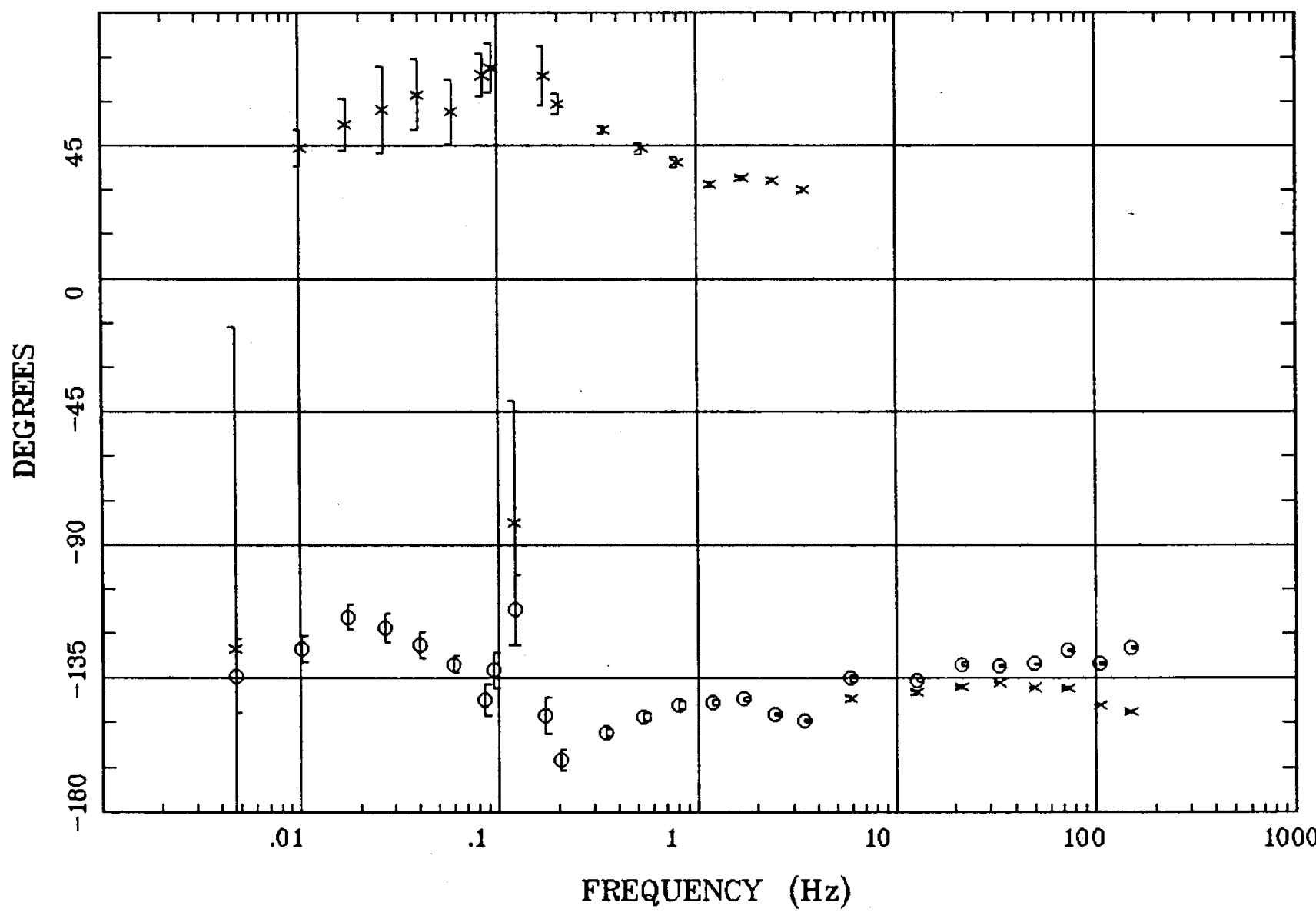

Client:

Remote:

Acquired:

Survey Co:
Rotation:

Filename: NNR01

Channels: Ch1 Ch2 Ch3 Ch4 Ch5 Ch6 Ch7

Plotted: 15:29 Dec 11, 2000

< EMI - ElectroMagnetic Instruments 


\section{ROTATION ANGLE}

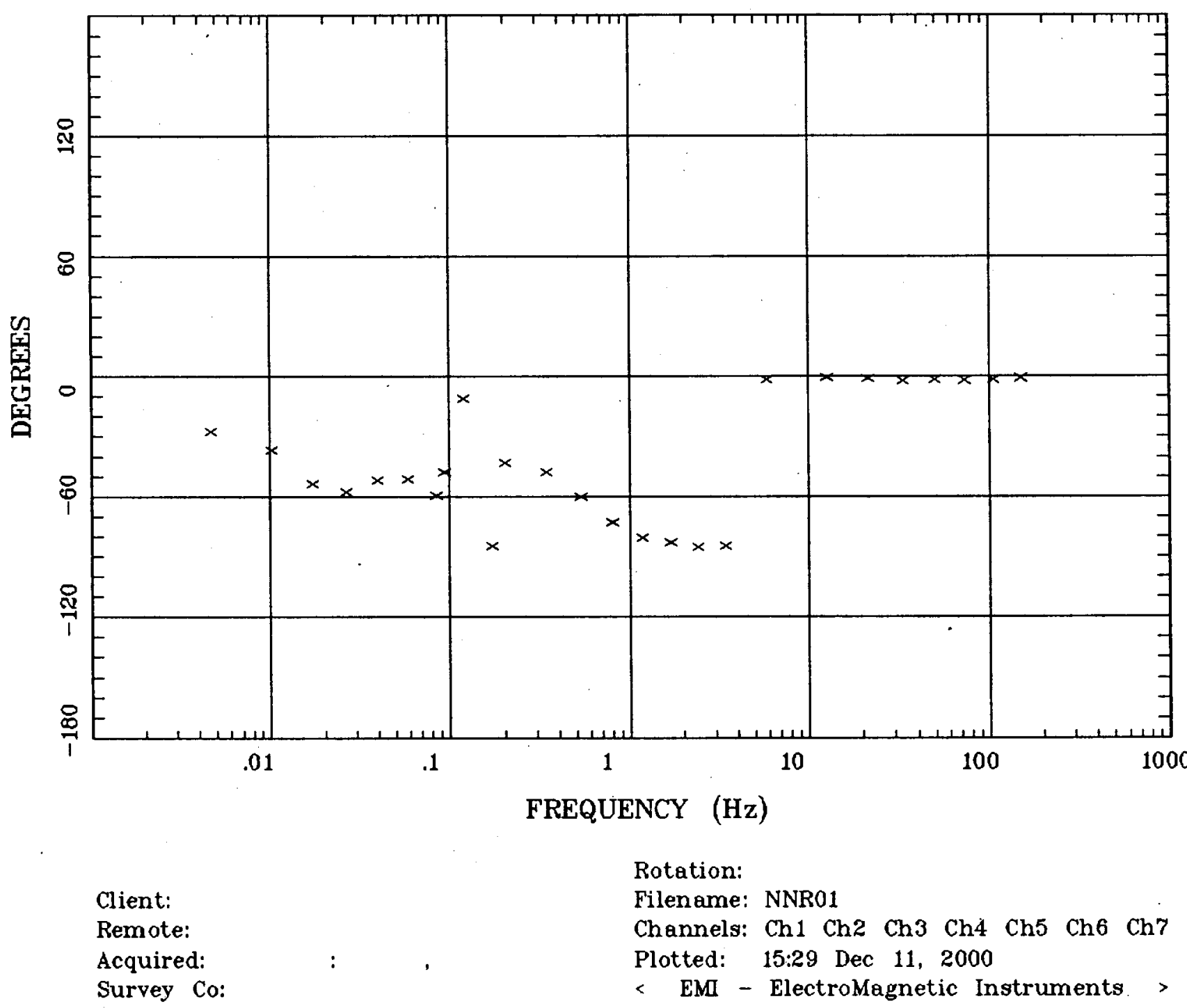


Station 1

IMPEDANCE SKEW

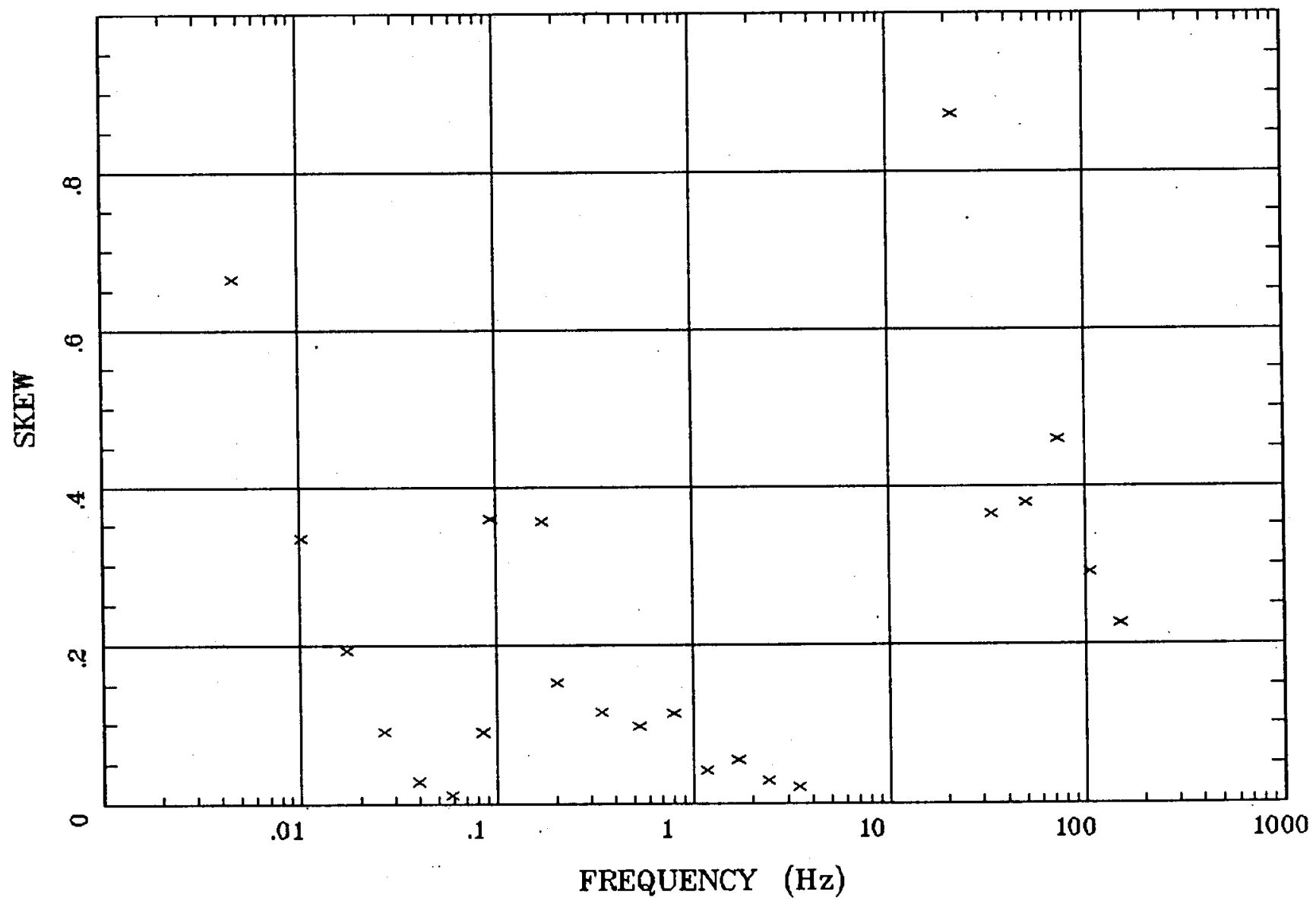

Client:

Remote:

Acquired: Survey Co:
Rotation:

Filename: NNR01

Channels: Ch1 Ch2 Ch3 Ch4 Ch5 Ch6 Ch7 Plotted: 15:29 Dec 11, 2000

< EMI - ElectroMagnetic Instruments 


\section{Station 1}

E MULT Coh.

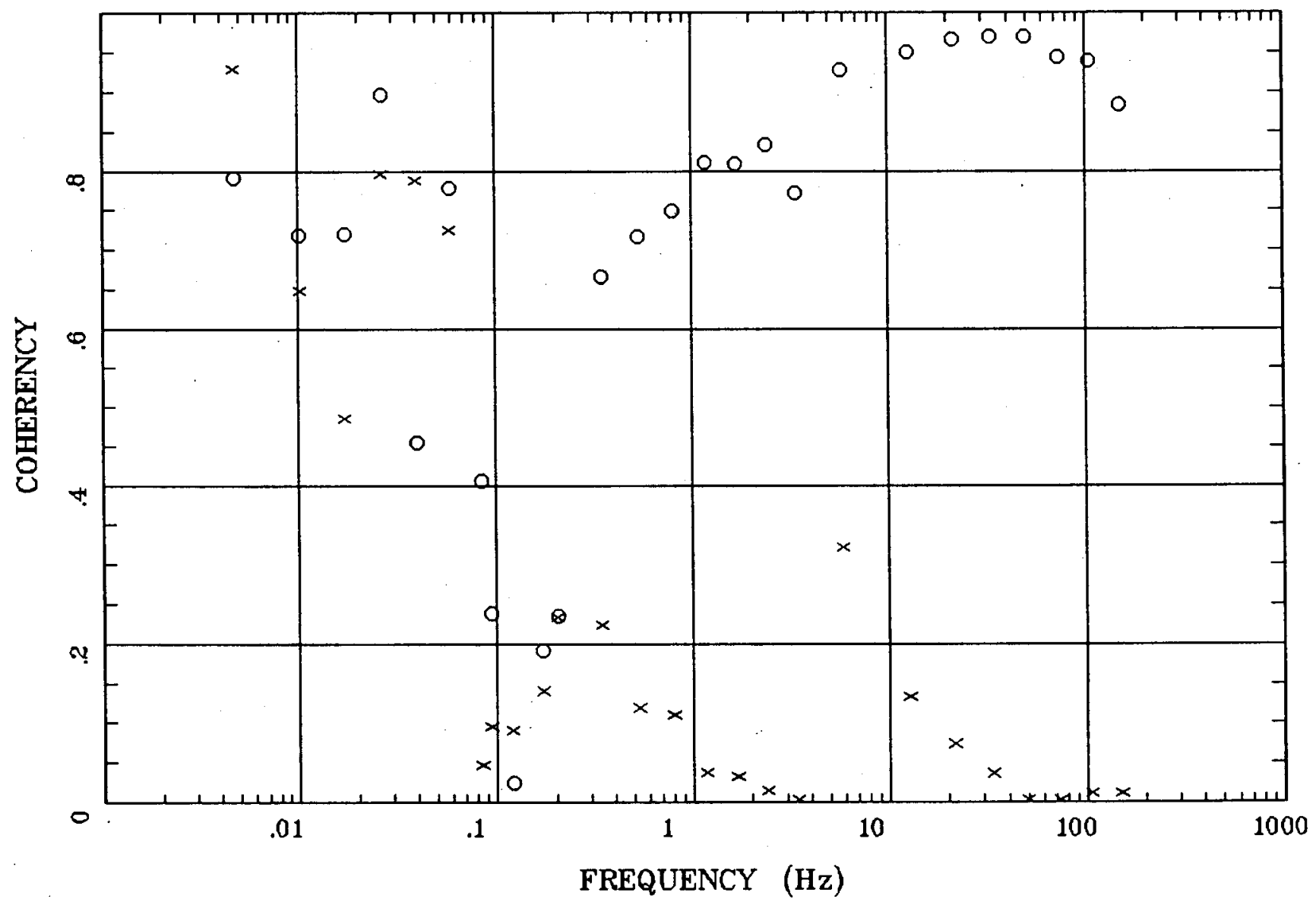

Client:

Remote:

Acquired:

Survey Co
Rotation:

Filename: NNR01

Channels: Ch1 Ch2 Ch3 Ch4 Ch5 Ch6 Ch7

Plotted: 15:29 Dec 11, 2000

< EMI - ElectroMagnetic Instruments 


\section{POLAR PLOTS}

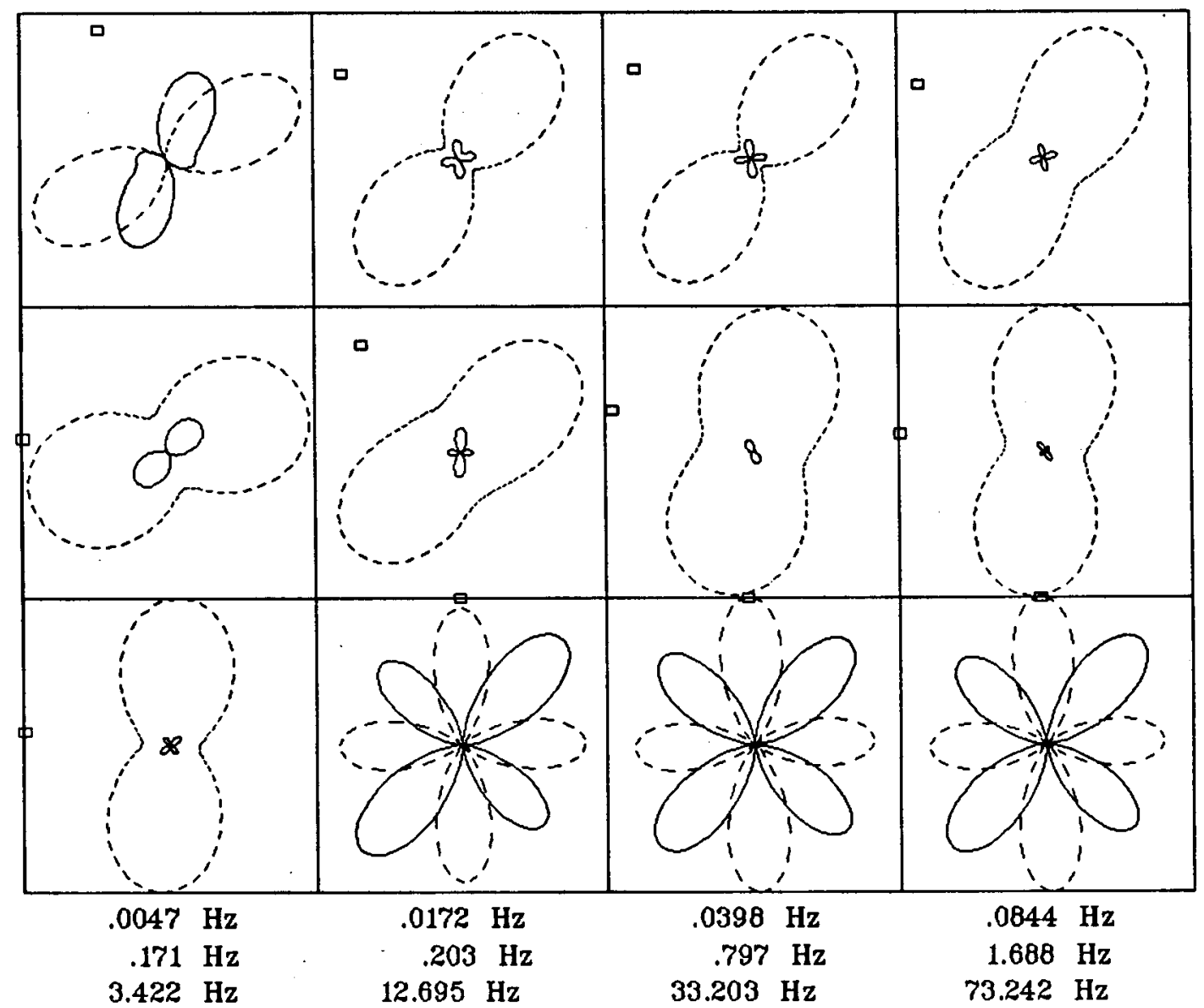

\section{Client:}

Remote:

Acquired:

Survey Co:
Rotation:

Filename: NNRO1

Channels: Ch1 Ch2 Ch3 Ch4 Ch5 Ch6 Ch7

Plotted: 15:29 Dec 11, 2000

< EMI - ElectroMagnetic Instruments . > 
APPARENT RESISTIVITY

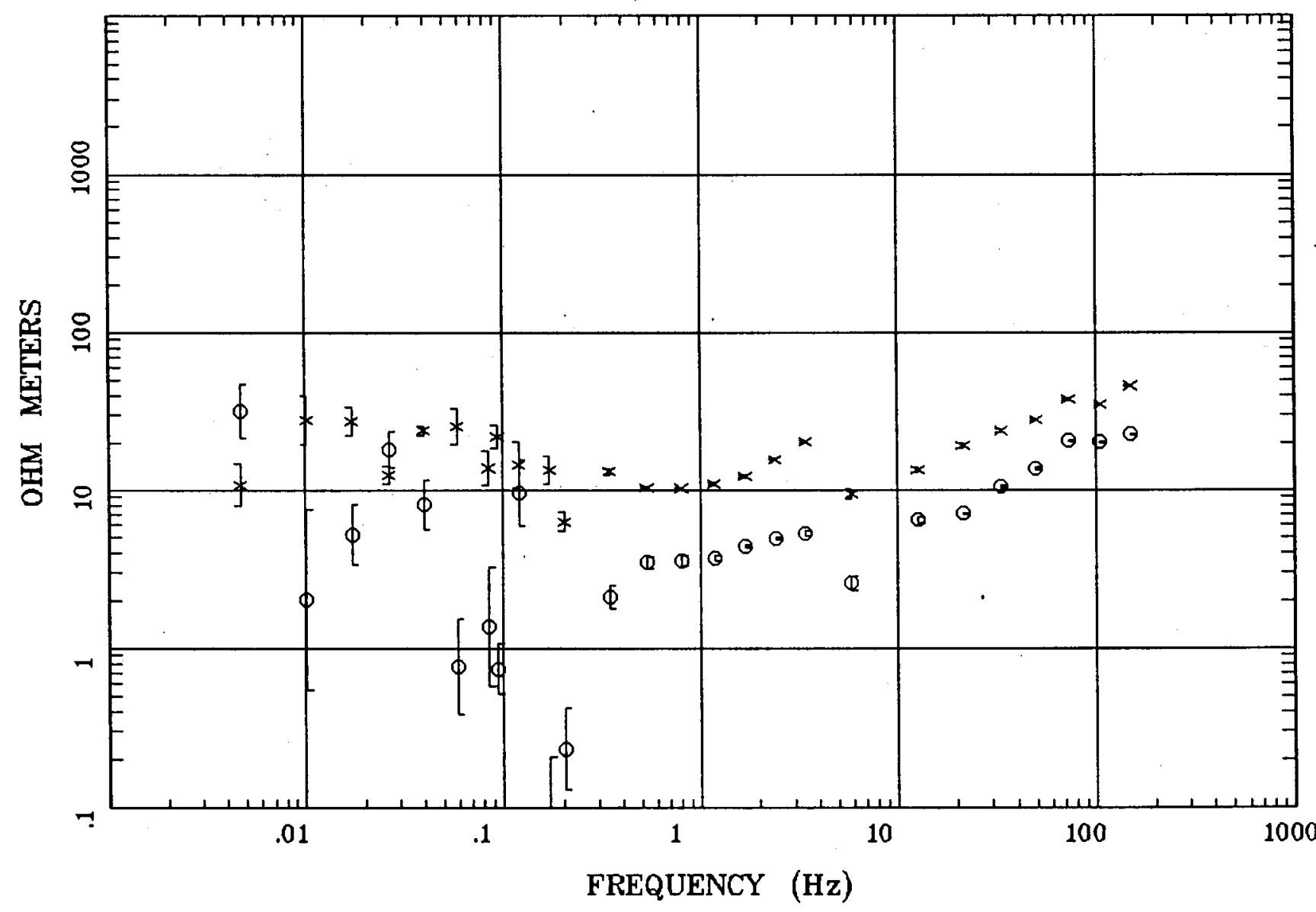

Client:

Remote:

Acquired: Survey Co:
Rotation:

Filename: NNR02

Channels: Ch1 Ch2 Ch3 Ch4 Ch5 Ch6 Ch7 Plotted: 15:34 Dec 11, 2000

< EMI - ElectroMagnetic Instruments 
Station 2

\section{IMPEDANCE PHASE}

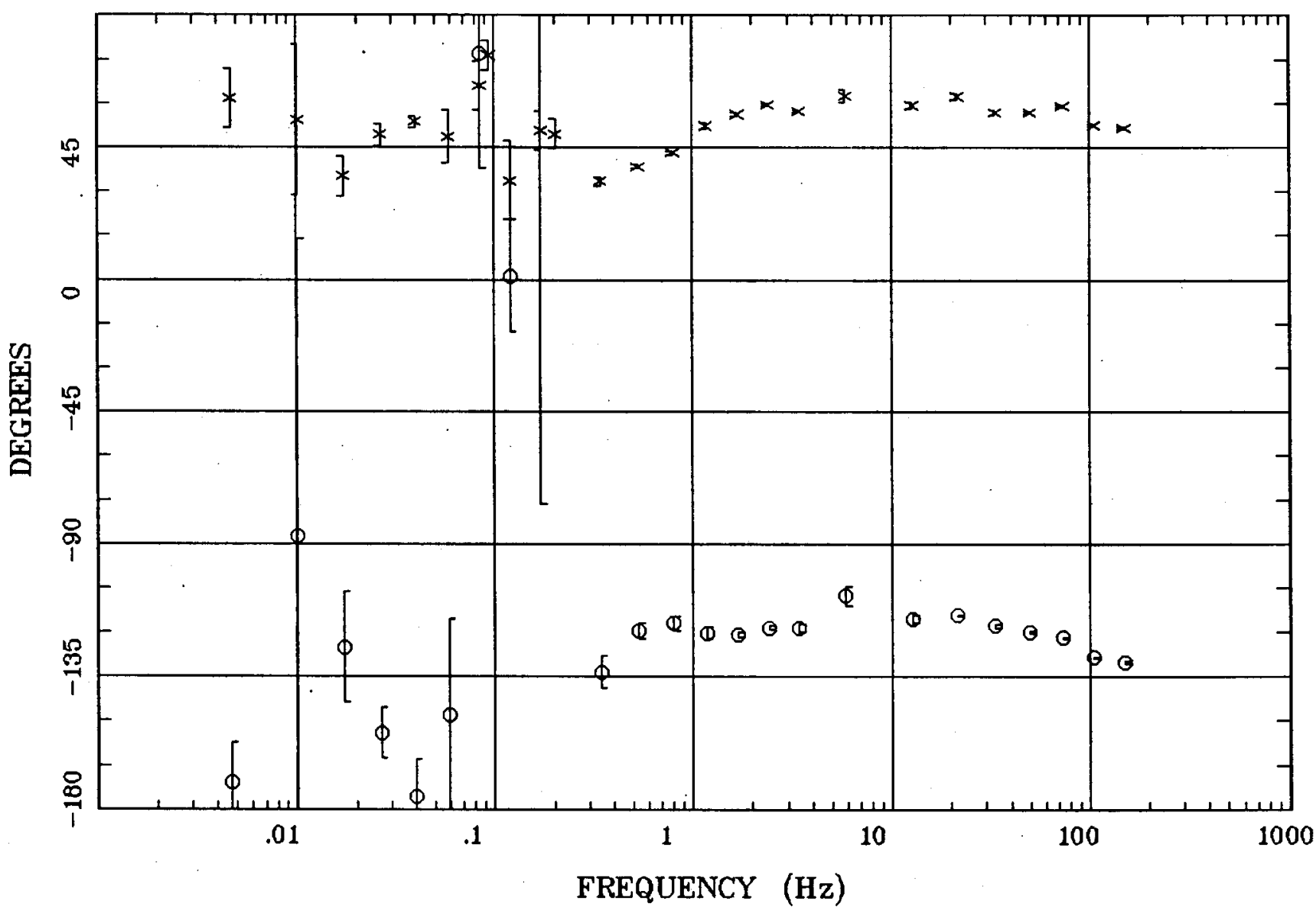

Client:

Remote: Acquired: Survey Co:
Rotation:

Filename: NNR02

Channels: Ch1 Ch2 Ch3 Ch4 Ch5 Ch6 Ch7

Plotted: 15:34 Dec 11, 2000

$<$ EMI - ElectroMagnetic Instruments 


\section{ROTATION ANGLE}

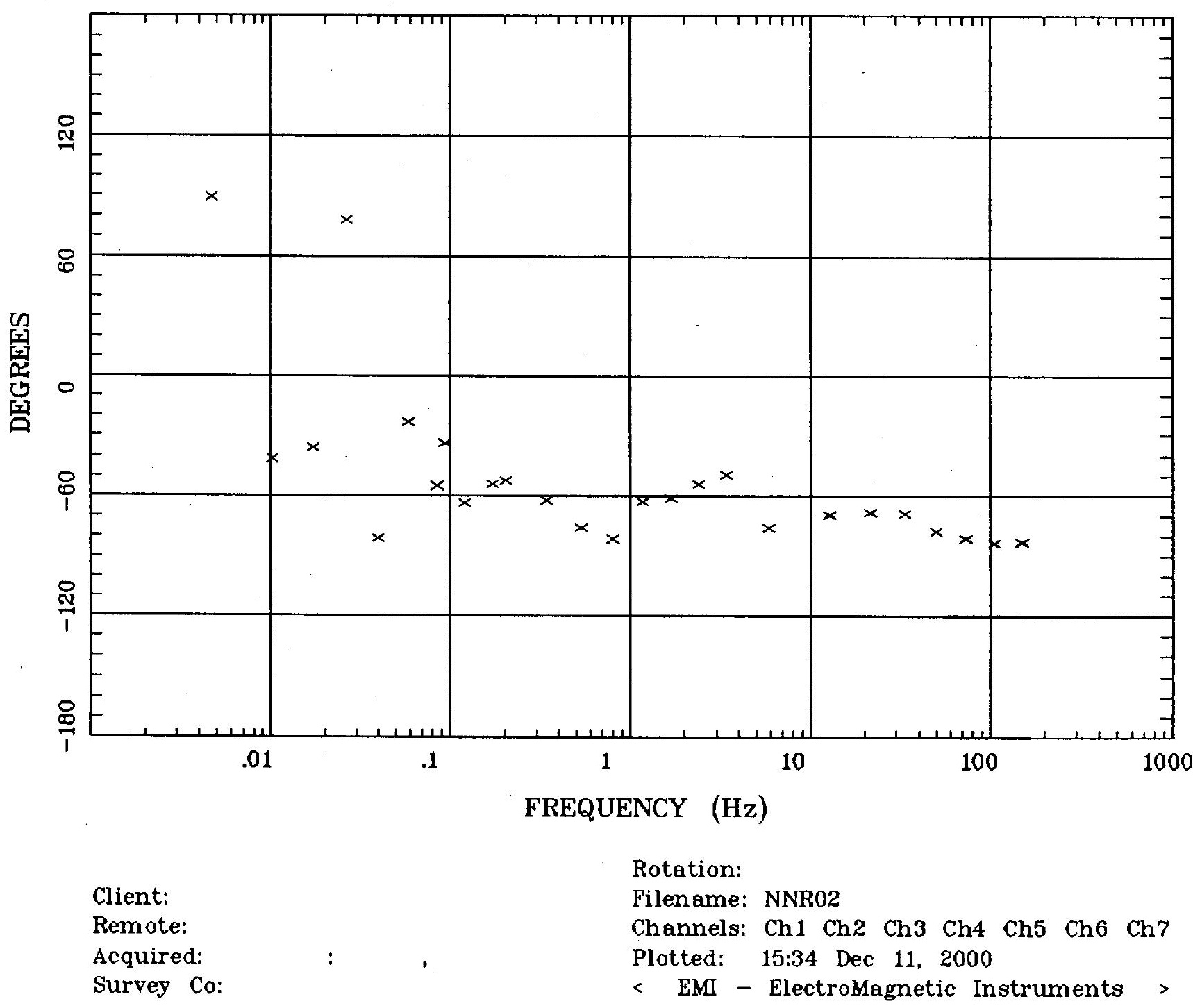




\section{Station 2}

\section{IMPEDANCE SKEW}

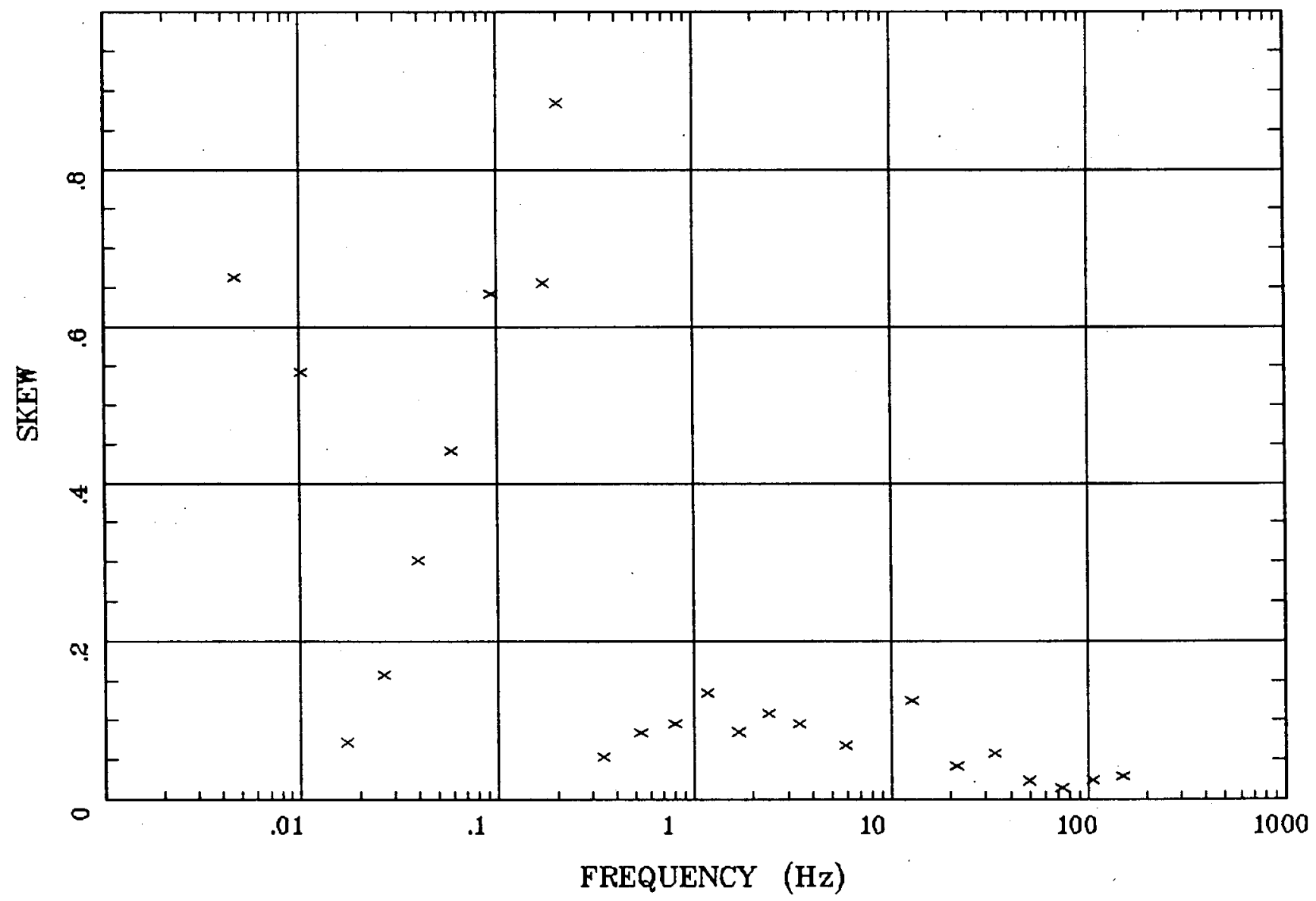

Client:

Remote:

Acquired:

Survey Co:
Rotation:

Filename: NNR02

Channels: Ch1 Ch2 Ch3 Ch4 Ch5 Ch6 Ch7

Plotted: 15:34 Dec 11, 2000

< EMI - ElectroMagnetic Instruments 
E MULT Coh.

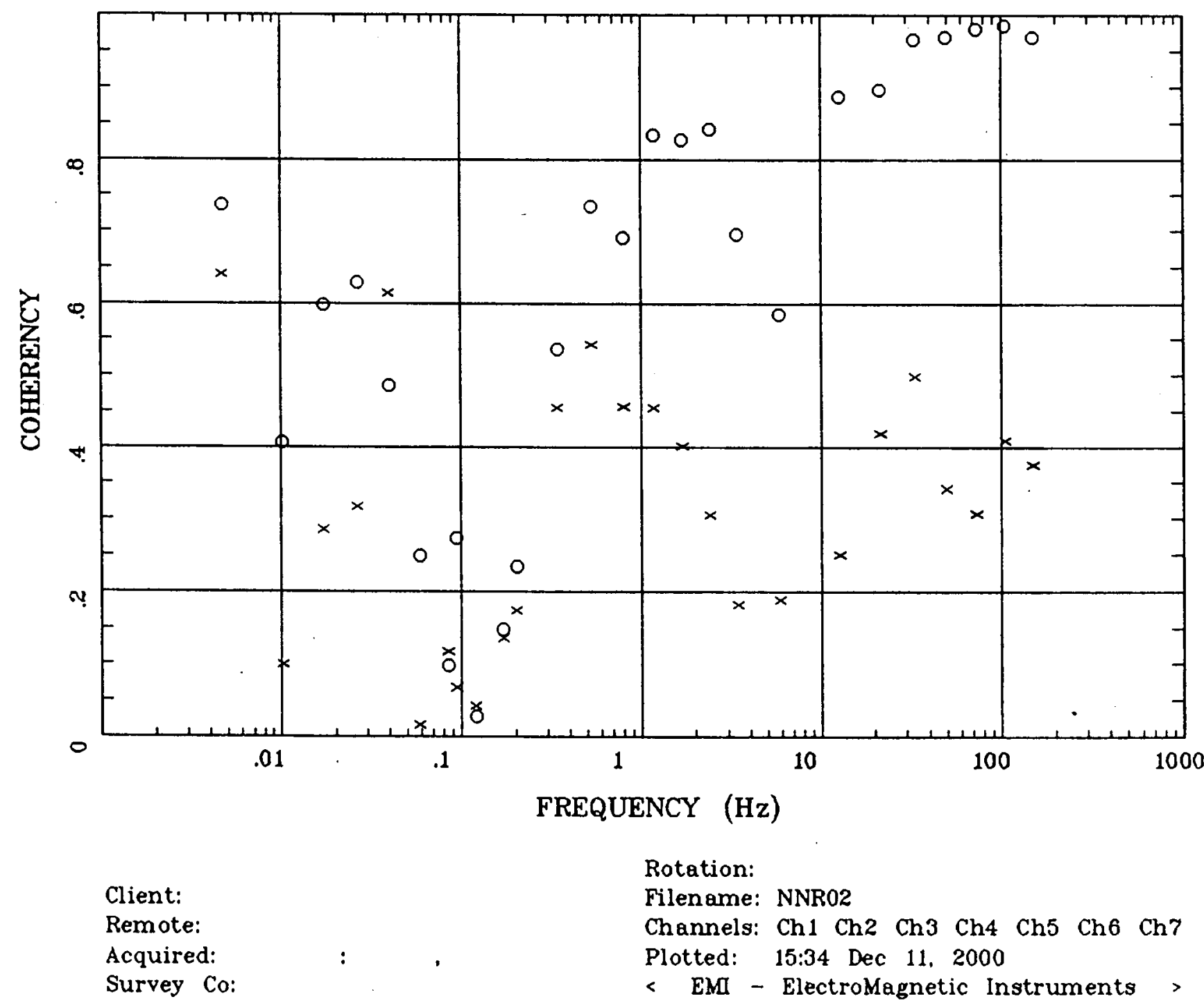


Station 2

POLAR PLOTS

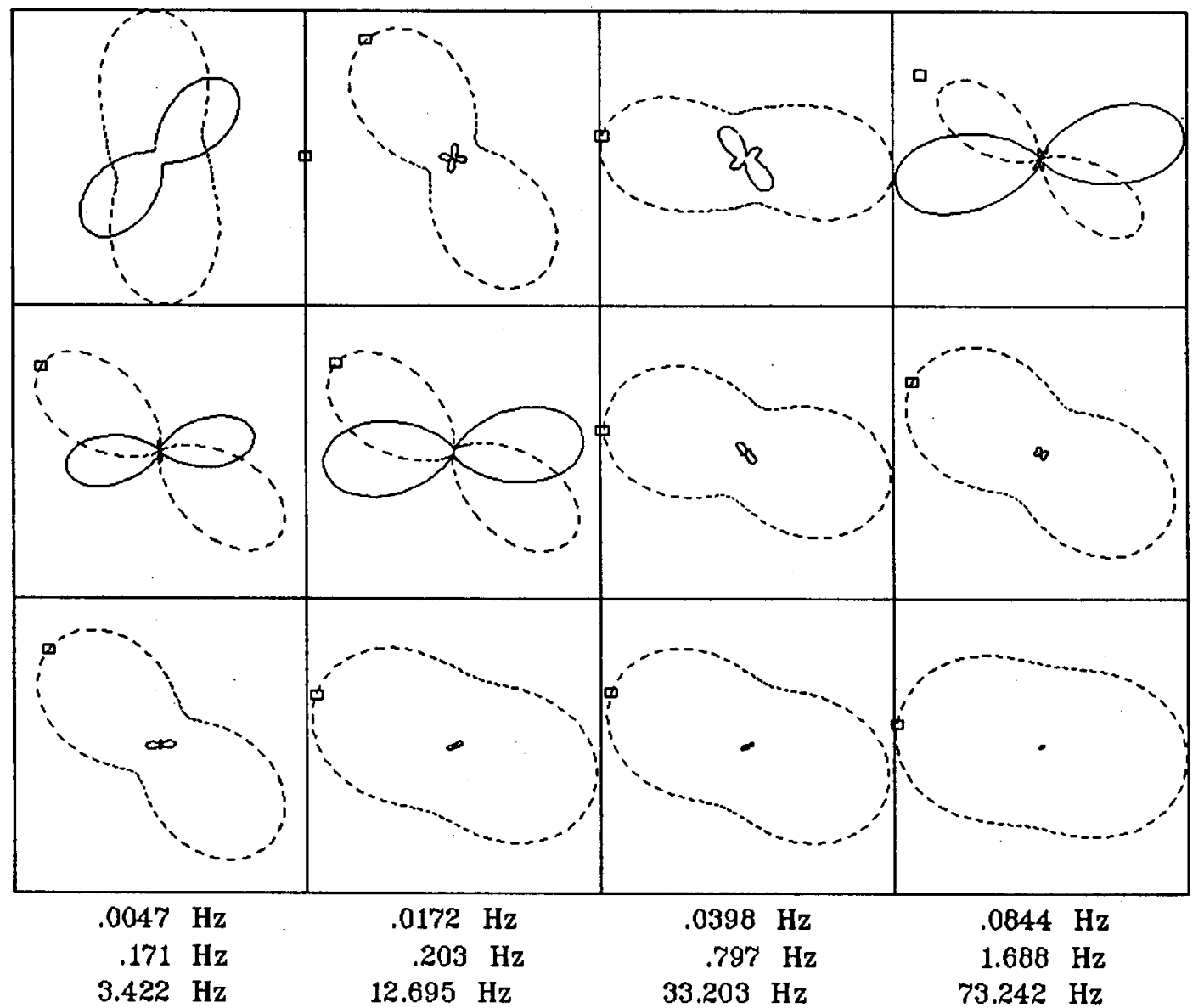

Client:

Remote:

Acquired:

Survey Co:

\section{Rotation:}

Filename: NNR02

Channels: Ch1 Ch2 Ch3 ch4 Ch5 Ch6 Ch7 Plotted: 15:34 Dec 11, 2000

< EMI - ElectroMagnetic Instruments 


\section{Station 105}

APPARENT RESISTIVITY

Crescent Valley, NV 100K

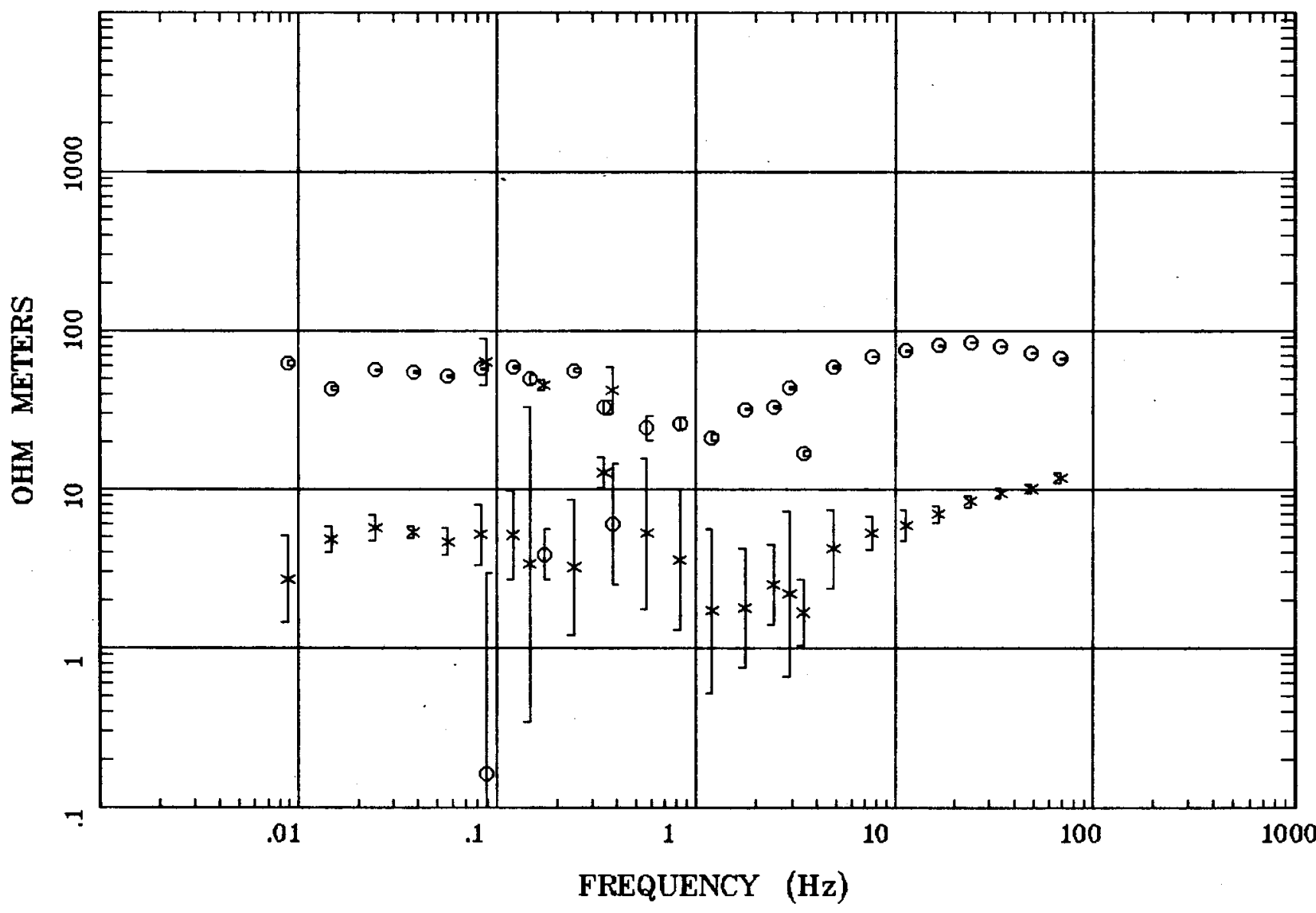

Client:

Remote: none

Acquired: 10:1 Aug 04, 2000

Survey Co:USGS
Rotation:

Filename: hr105c.avg

Channels: Ch1 Ch2 Ch3 Ch4 Ch5 Ch3 Ch4

Plotted: 15:24 Jan 24, 2001

$<$ EMI - ElectroMagnetic Instruments > 


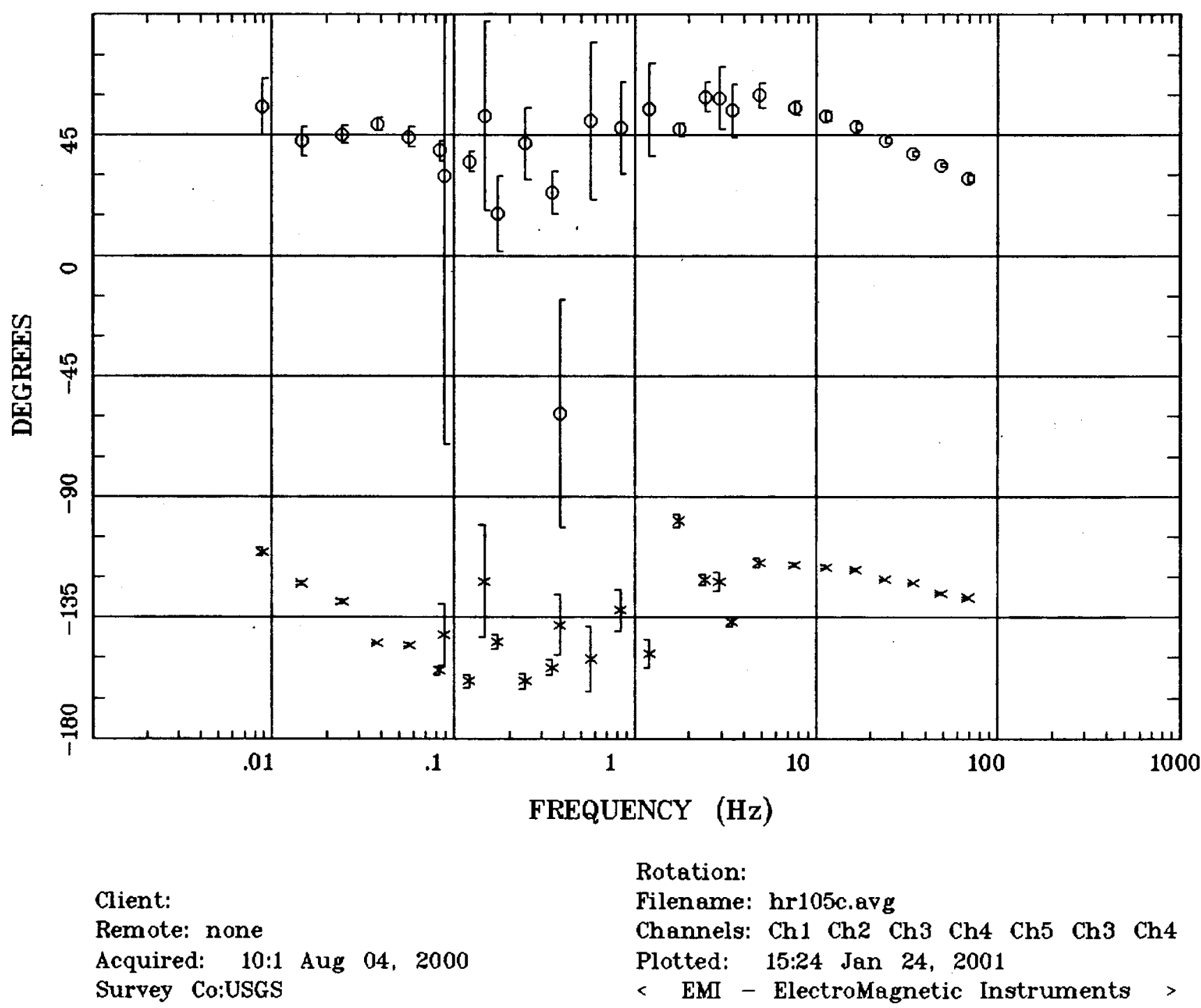




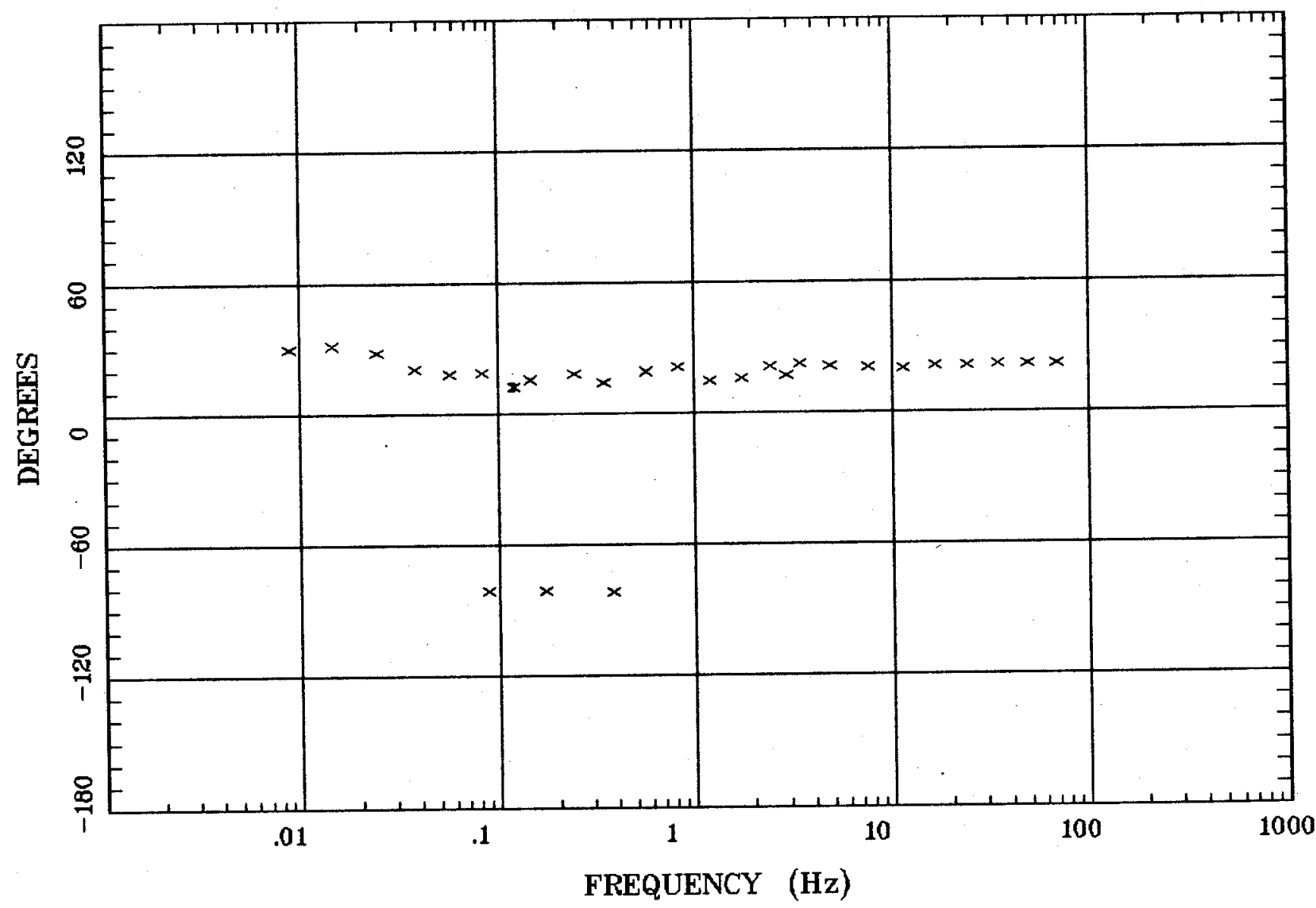

Client:

Remote: none

Acquired: 10:1 Aug 04, 2000 Survey Co:USGS
Rotation:

Filename: hr105c.avg

Channels: Ch1 Ch2 Ch3 ch4 Ch5 Ch3 Ch4 Plotted: 15:24 Jan 24, 2001

< EMI - ElectroMagnetic Instruments 


\section{Station 105}

IMPEDANCE SKEW

Crescent Valley, NV 100K

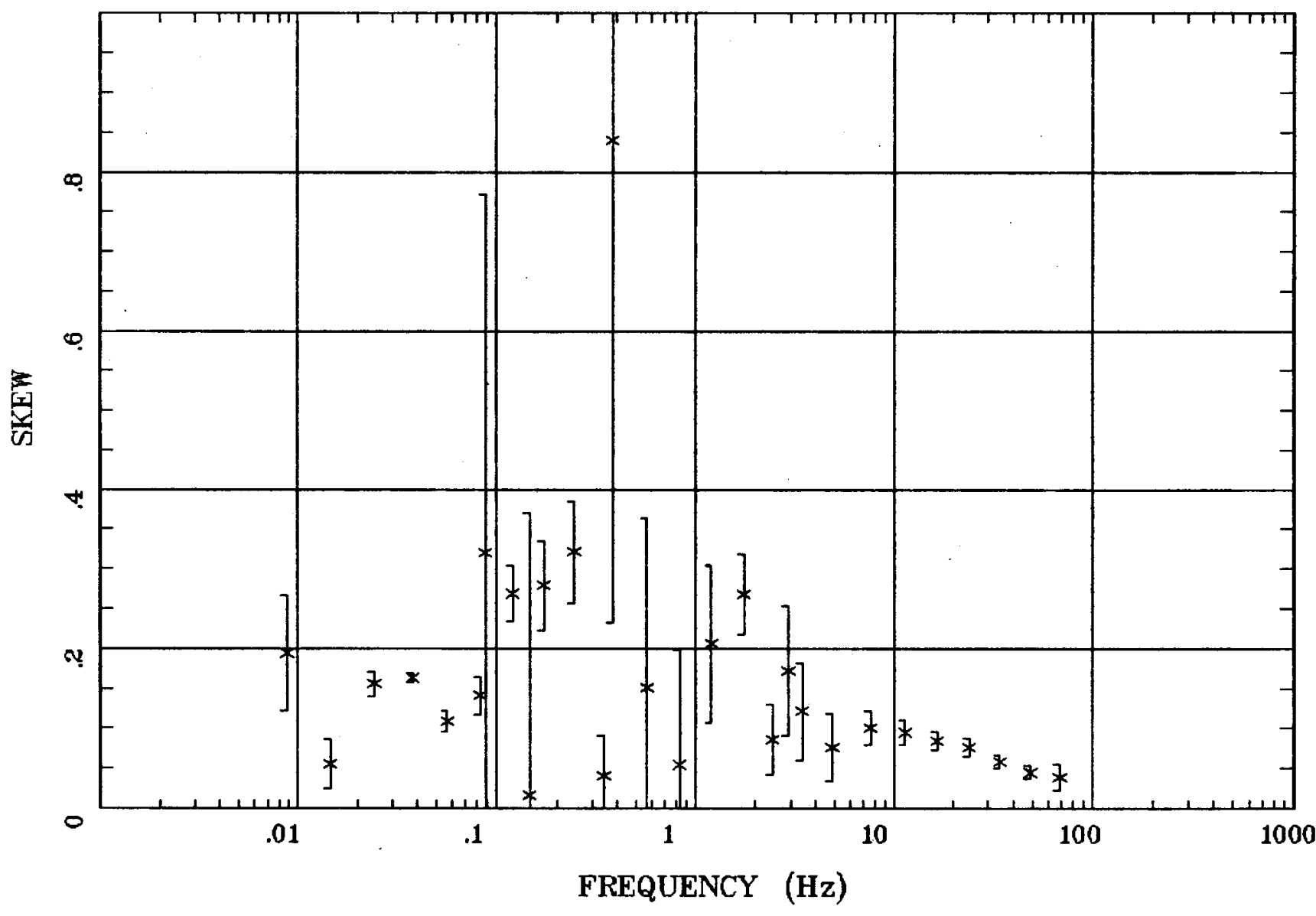

Client:

Remote: none

Acquired: 10:1 Aug 04, 2000 Survey Co:USGS
Rotation:

Filename: hr105c.avg

Channels: Ch1 Ch2 Ch3 Ch4 Ch5 Ch3 Ch4

Plotted: 15:24 Jan 24, 2001

$<$ EMI - ElectroMagnetic Instruments > 
Station 105

E MULT Coh.

Crescent Valley, NV 100K

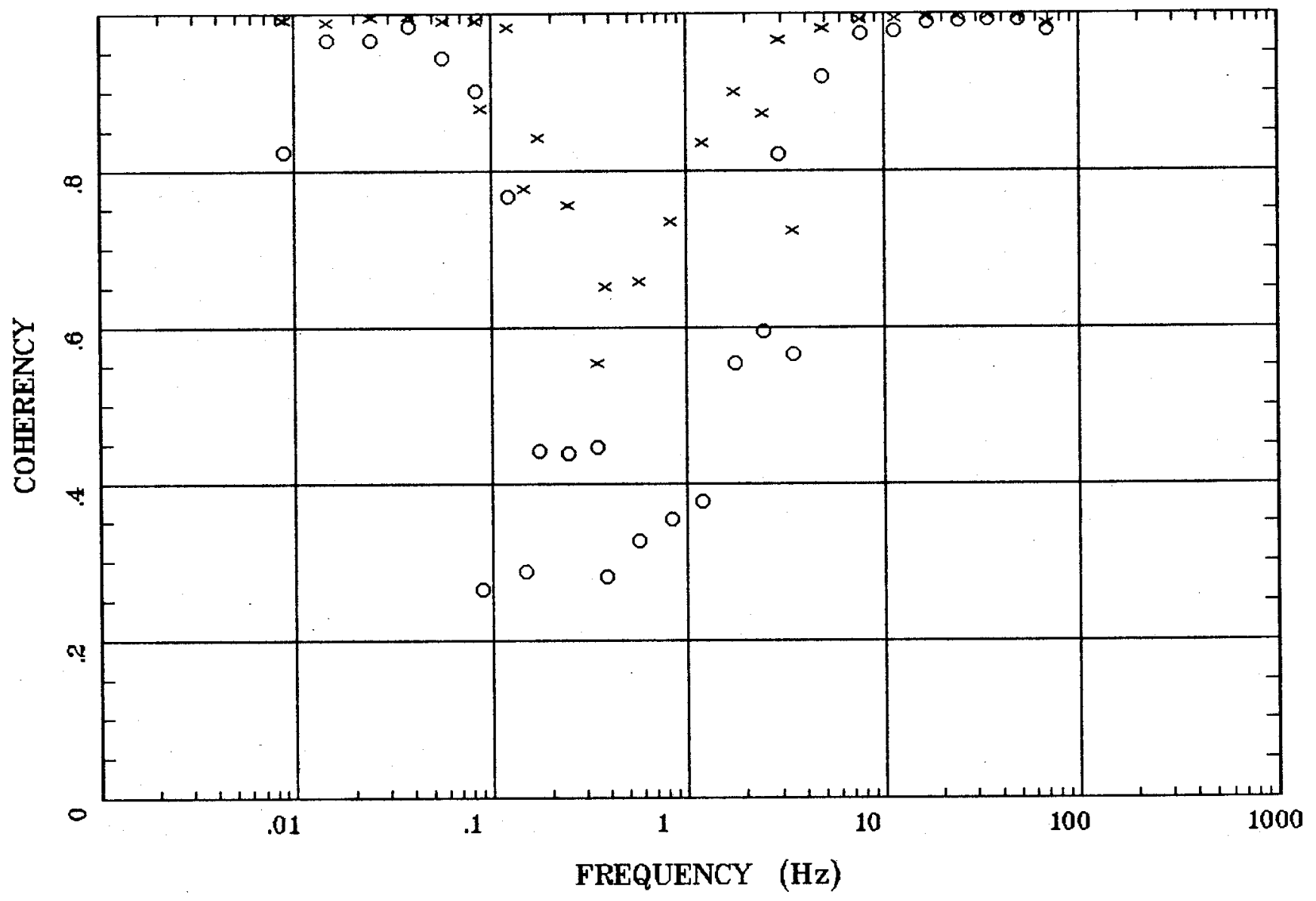

Client:

Remote: none

Acquired: 10:1 Aug 04, 2000 Survey Co:USGS
Rotation:

Filename: hr105c.avg

Channels: Ch1 Ch2 Ch3 Ch4 Ch5 Ch3 Ch4

Plotted: 15:24 Jan 24, 2001

< EMI - ElectroMagnetic Instruments 


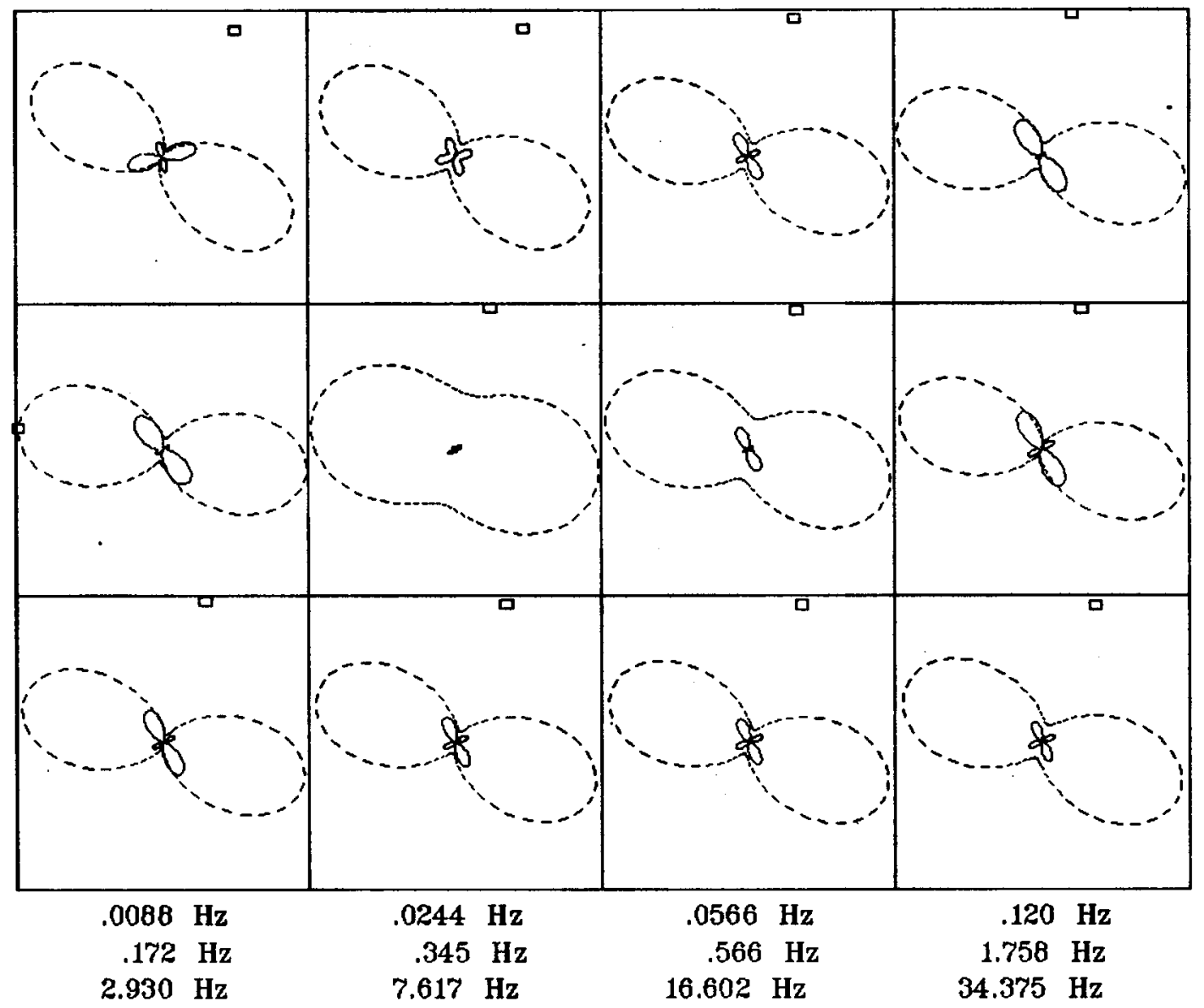

Client:

Remote: none

Acquired: 10:1 Aug 04, 2000 Survey Co:USGS

\section{Rotation:}

Filename: hr105c.avg

Channels: Ch1 Ch2 Ch3 Ch4 Ch5 Ch3 Ch4 Platted: 15:24 Jan 24, 2001

< EMI - ElectroMagnetic Instruments > 


\section{Station 105}

\section{TIPPER MAGNITUDE}

Crescent Valley, NV 100K

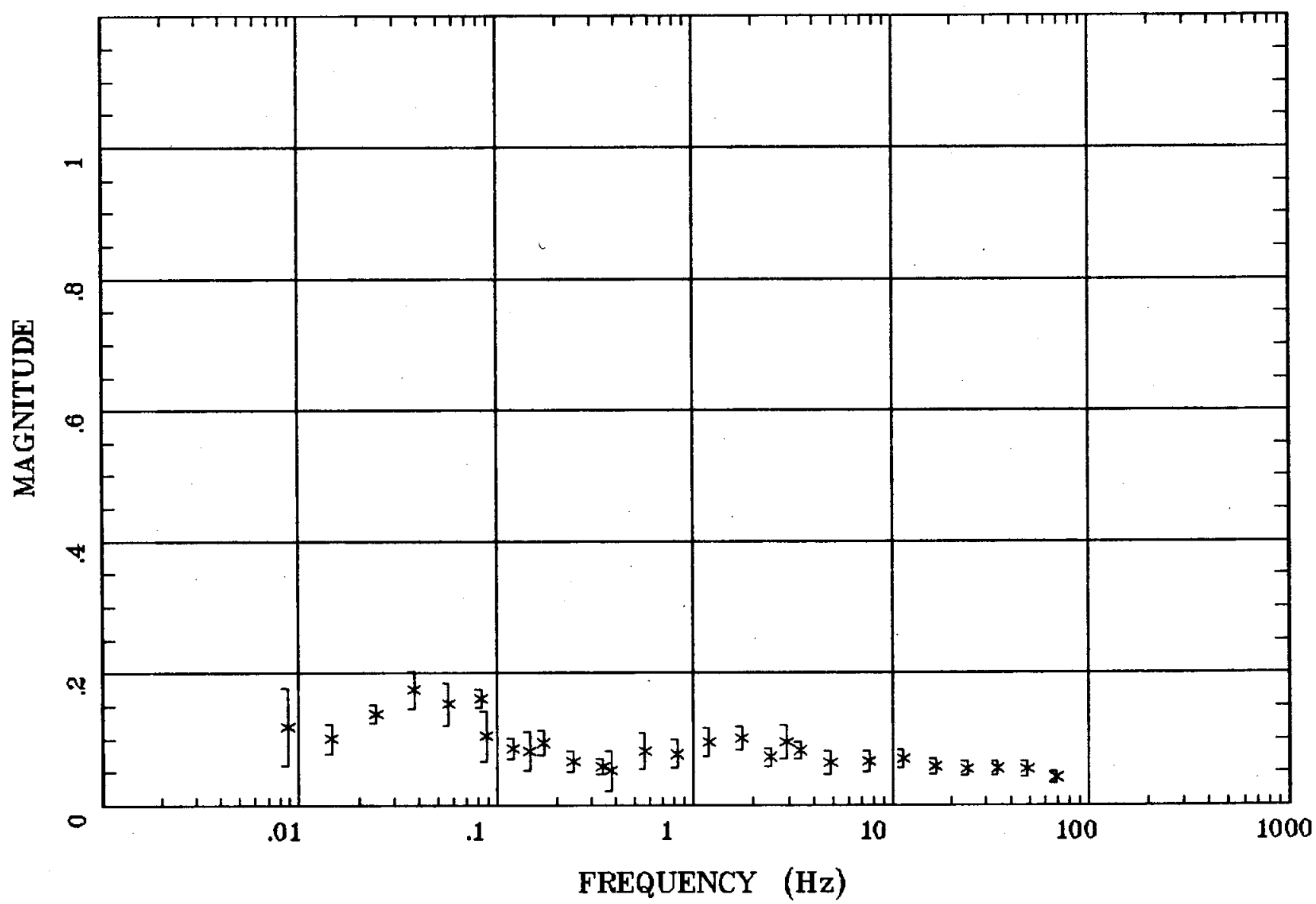

Client:

Remote: none

Acquired: 10:1 Aug 04, 2000

Survey Co:USGS
Rotation:

Filename: hr105c.avg

Channels: Ch1 Ch2 Ch3 Ch4 Ch5 Ch3 Ch4

Plotted: 15:24 Jan 24, 2001

< EMI - ElectroMagnetic Instruments > 
Station 105

TIPPER STRIKE

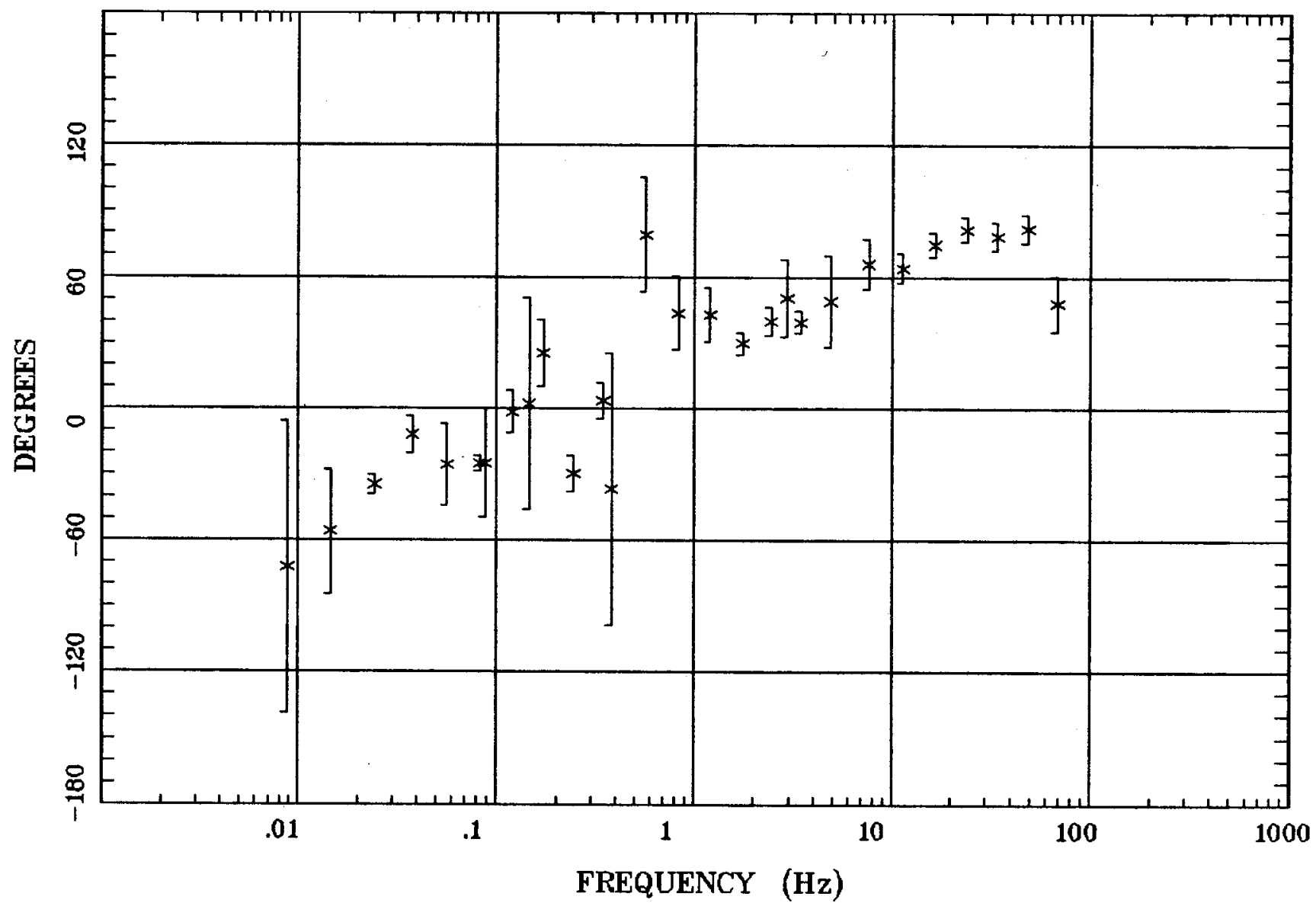

Client:

Remote: none

Acquired: $10: 1$ Aug 04, 2000 Survey Co:USGS
Crescent Valley, NV 100K

Rotation:

Filename: hr105c.avg

Channels: Ch1 Ch2 Ch3 Ch4 Ch5 Ch3 ch4

Plotted: 15:24 Jan 24, 2001

$<$ EMI - ElectroMagnetic Instruments 
Station 105

HzHx.x Coh HzHy.o Crescent Valley, NV 100K

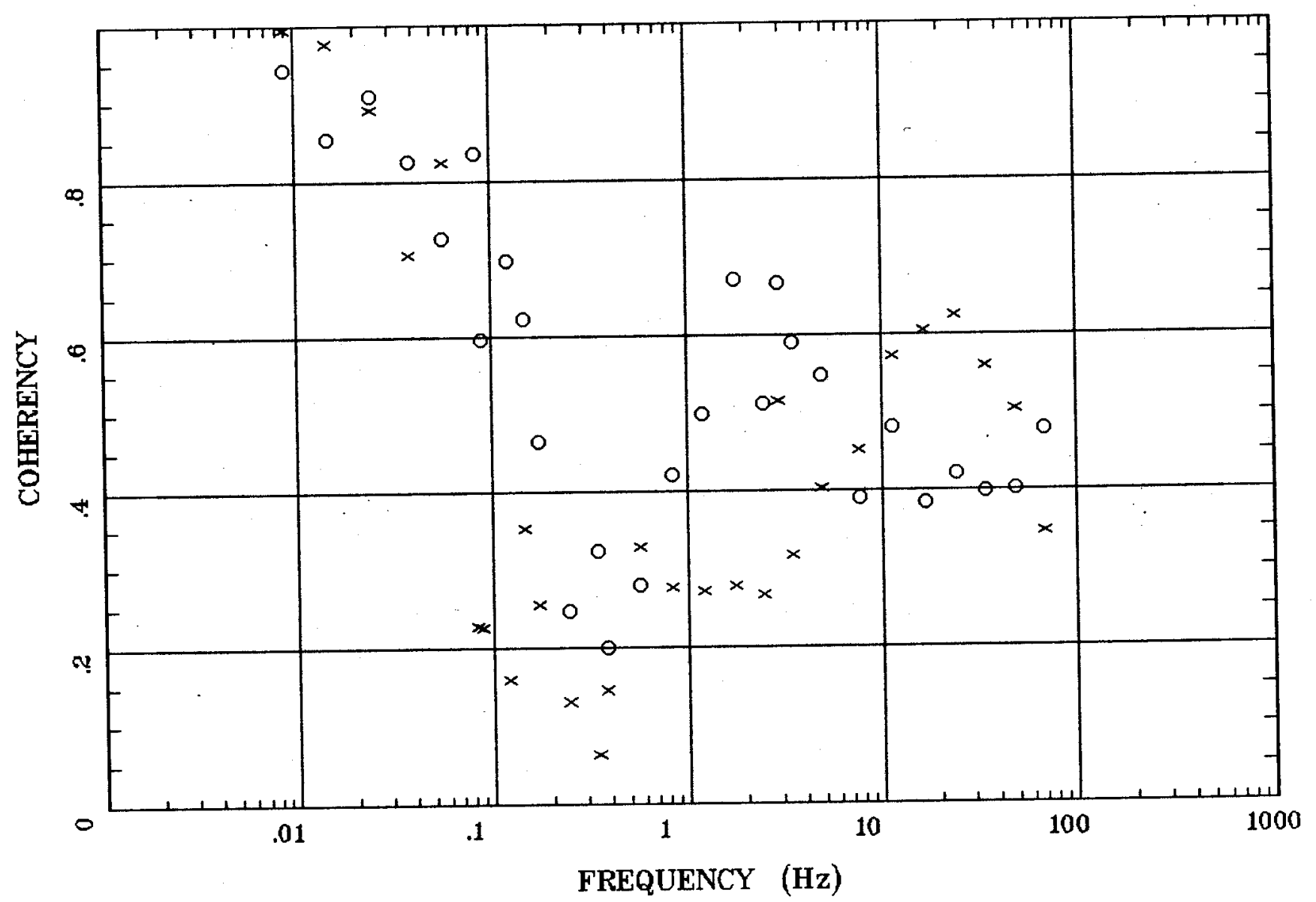

Client:

Remote: none

Acquired: 10:1 Aug 04, 2000

Survey Co:USGS

Rotation:

Filename: hr105c.avg

Channels: Ch1 Ch2 Ch3 Ch4 Ch5 Ch3 Ch4

Platted: 15:24 Jan 24, 2001

$<$ EMI - ElectroMagnetic Instruments > 
Station 5

APPARENT RESISTIVITY

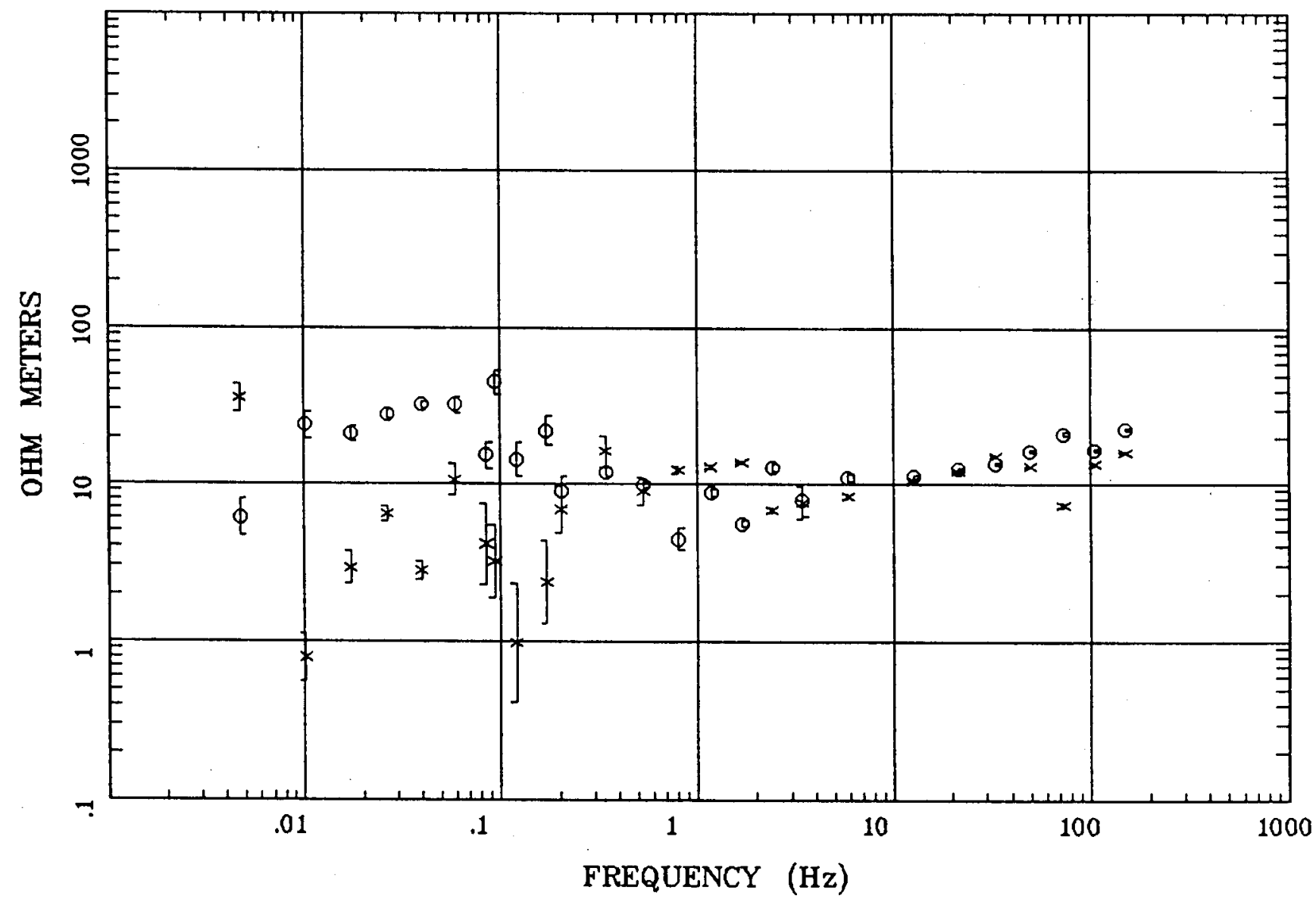

Client:

Remote:

Acquired:

Survey Co:
Rotation:

Filename: NNR05

Channels: Ch1 Ch2 Ch3 Ch4 Ch5 Ch6 Ch7

Plotted: 15:38 Dec 11, 2000

< EMI - ElectroMagnetic Instruments > 


\section{IMPEDANCE PHASE}

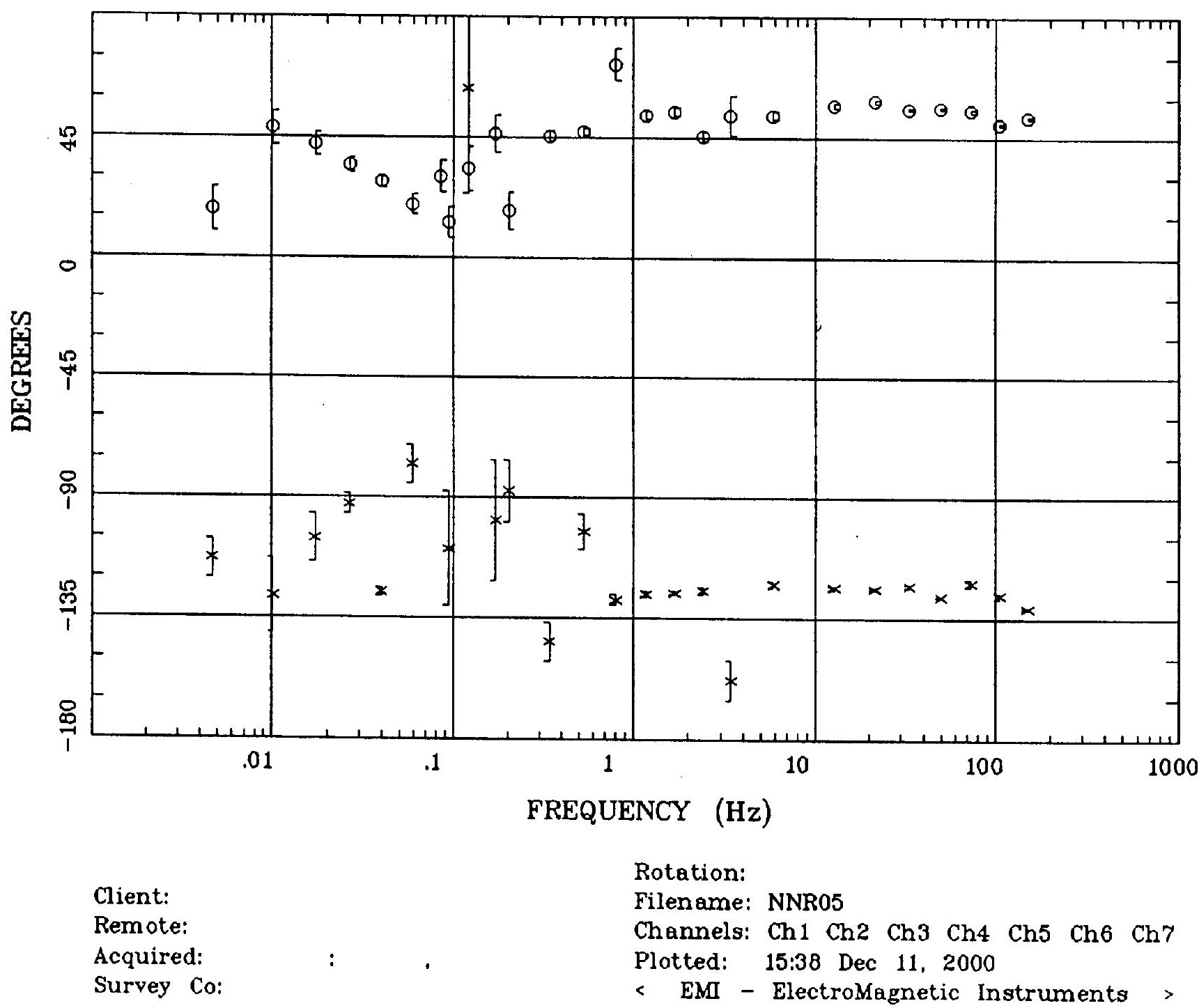




\section{ROTATION ANGLE}

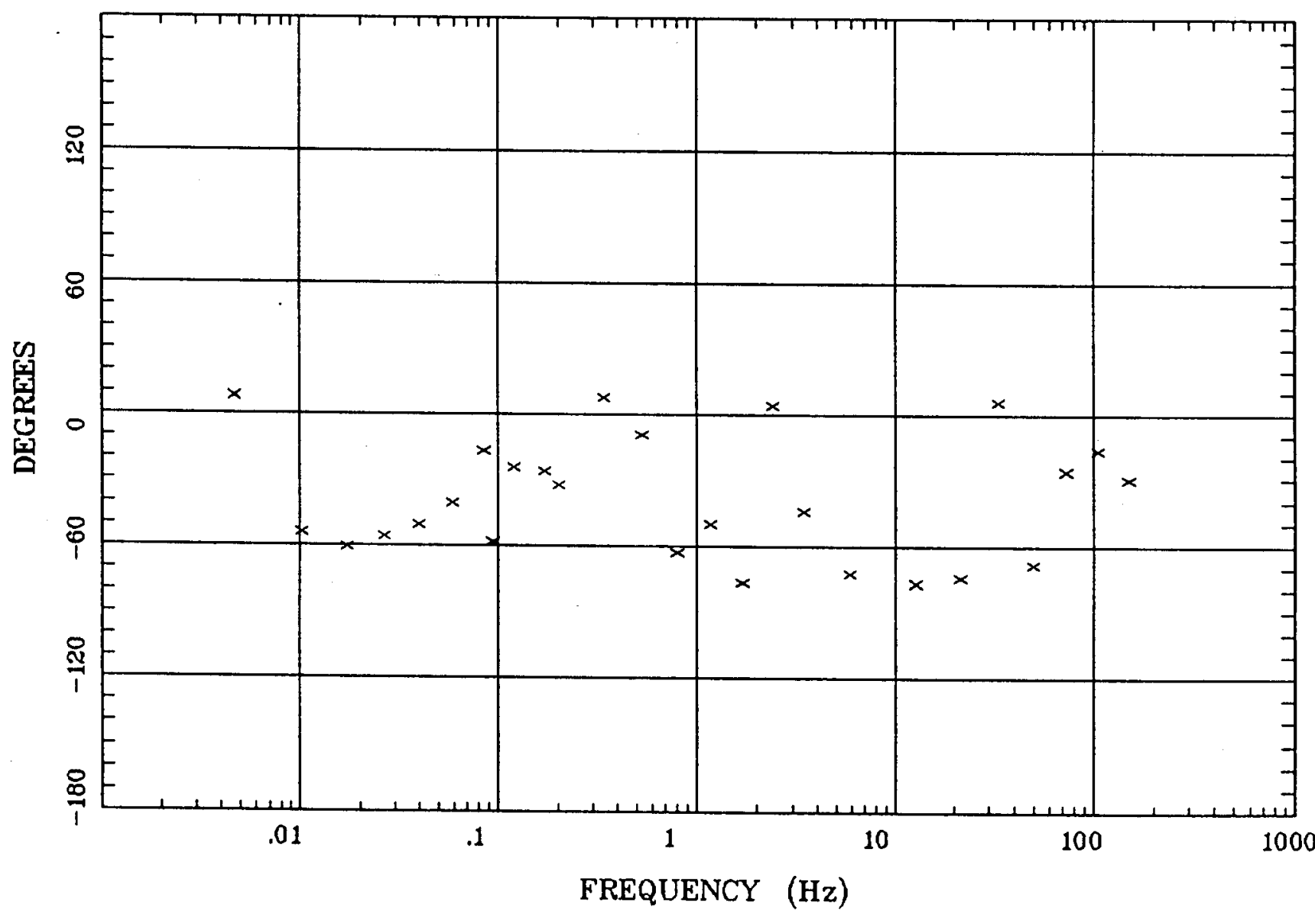

Client:

Remote:

Acquired:

Survey Co:
Rotation:

Filename: NNR05

Channels: Ch1 Ch2 Ch3 Ch4 Ch5 Ch6 Ch7

Plotted: 15:38 Dec 11, 2000

< EMI - ElectroMagnetic Instruments 


\section{Station 5}

\section{IMPEDANCE SKEW}

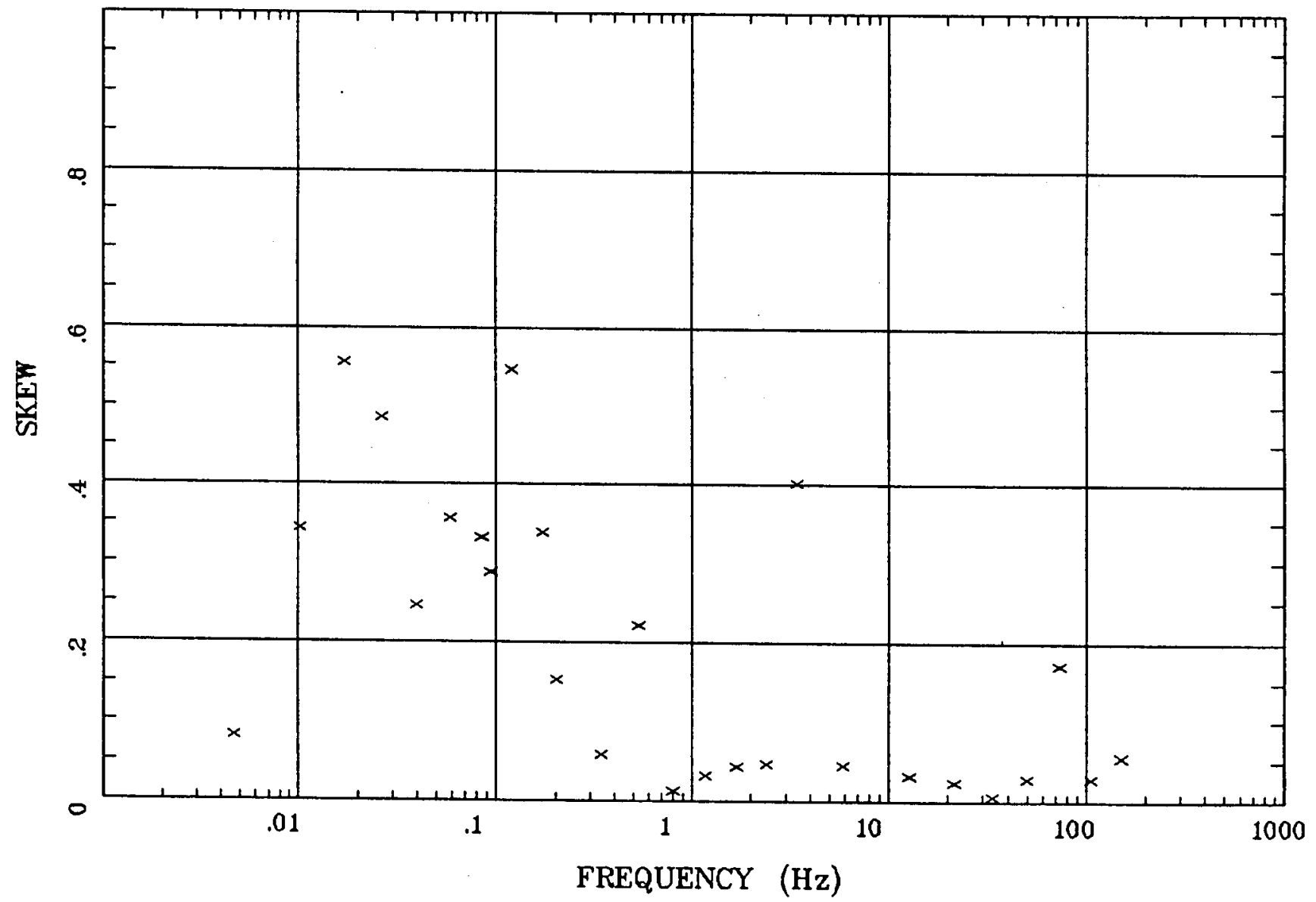

Client:

Remote:

Acquired:

Survey Co:
Rotation:

Filename: NNR05

Channels: Ch1 Ch2 Ch3 Ch4 Ch5 Ch6 Ch7

Plotted: 15:38 Dec 11, 2000

< EMI - ElectroMagnetic Instruments > 


\section{Station 5}

E MULT Coh.

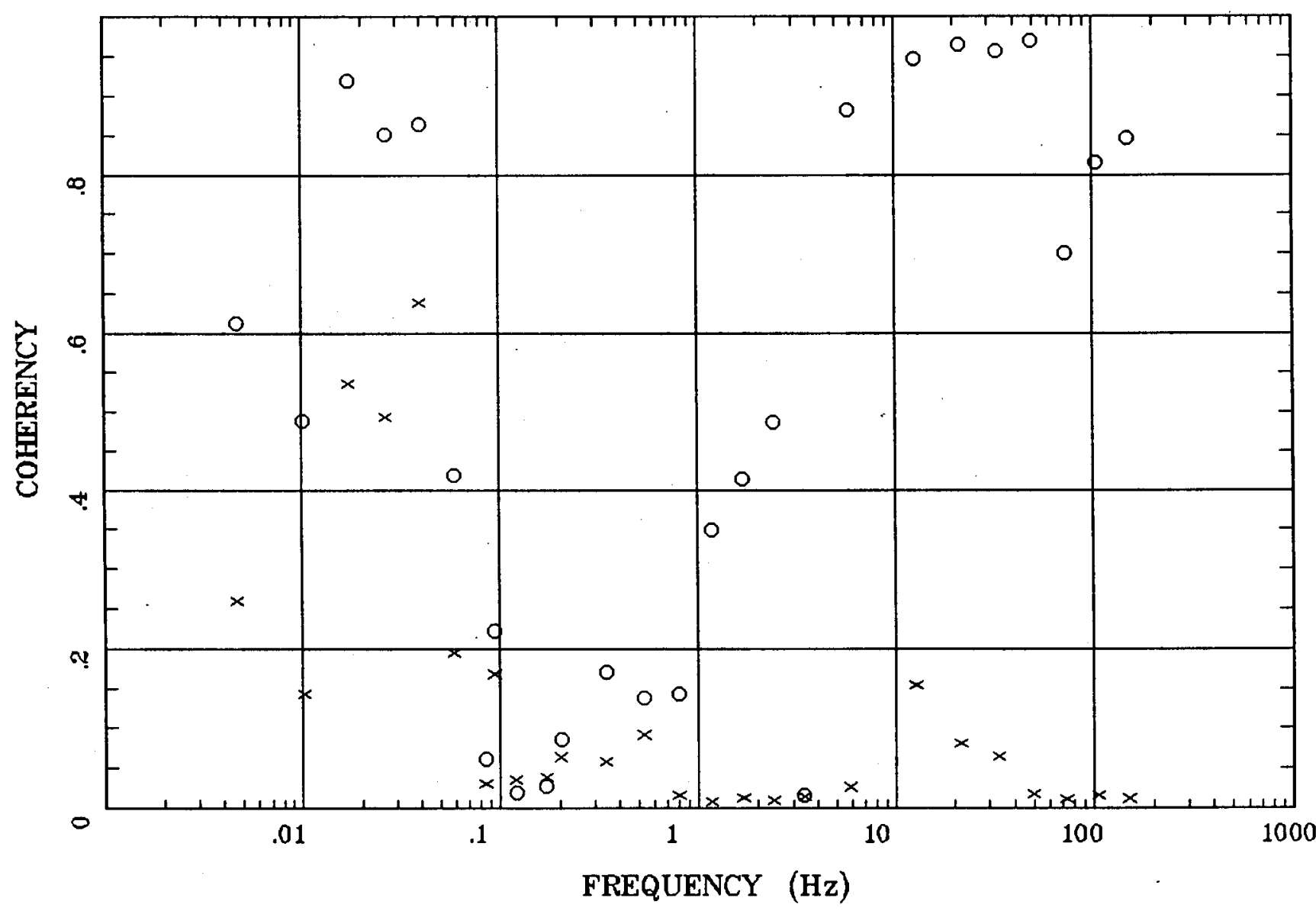

Client:

Remote:

Acquired:

Survey Co:
Rotation:

Filename: NNR05

Channels: Ch1 Ch2 Ch3 Ch4 Ch5 Ch6 Ch7

Plotted: 15:38 Dec 11, 2000

$<$ EMI - ElectroMagnetic Instruments 


\section{Station 5}

\section{POLAR PLOTS}

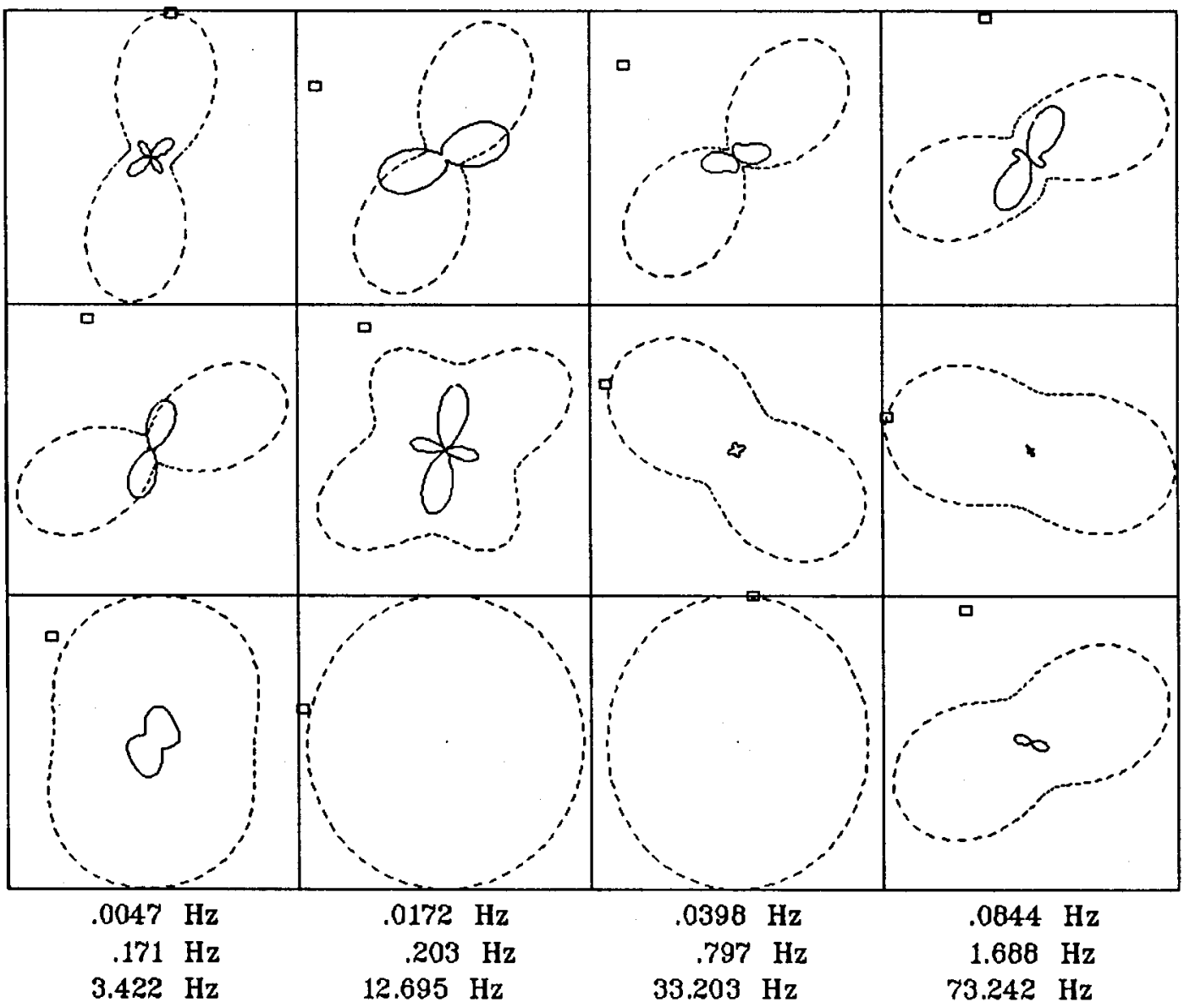

Client:

Ratation

Remote:

Filename: NNR05

Channels: Ch1 Ch2 Ch3 Ch4 Ch5 Ch6 Ch7

Plotted: 15:38 Dec 11, 2000 


\section{APPARENT RESISTIVITY}

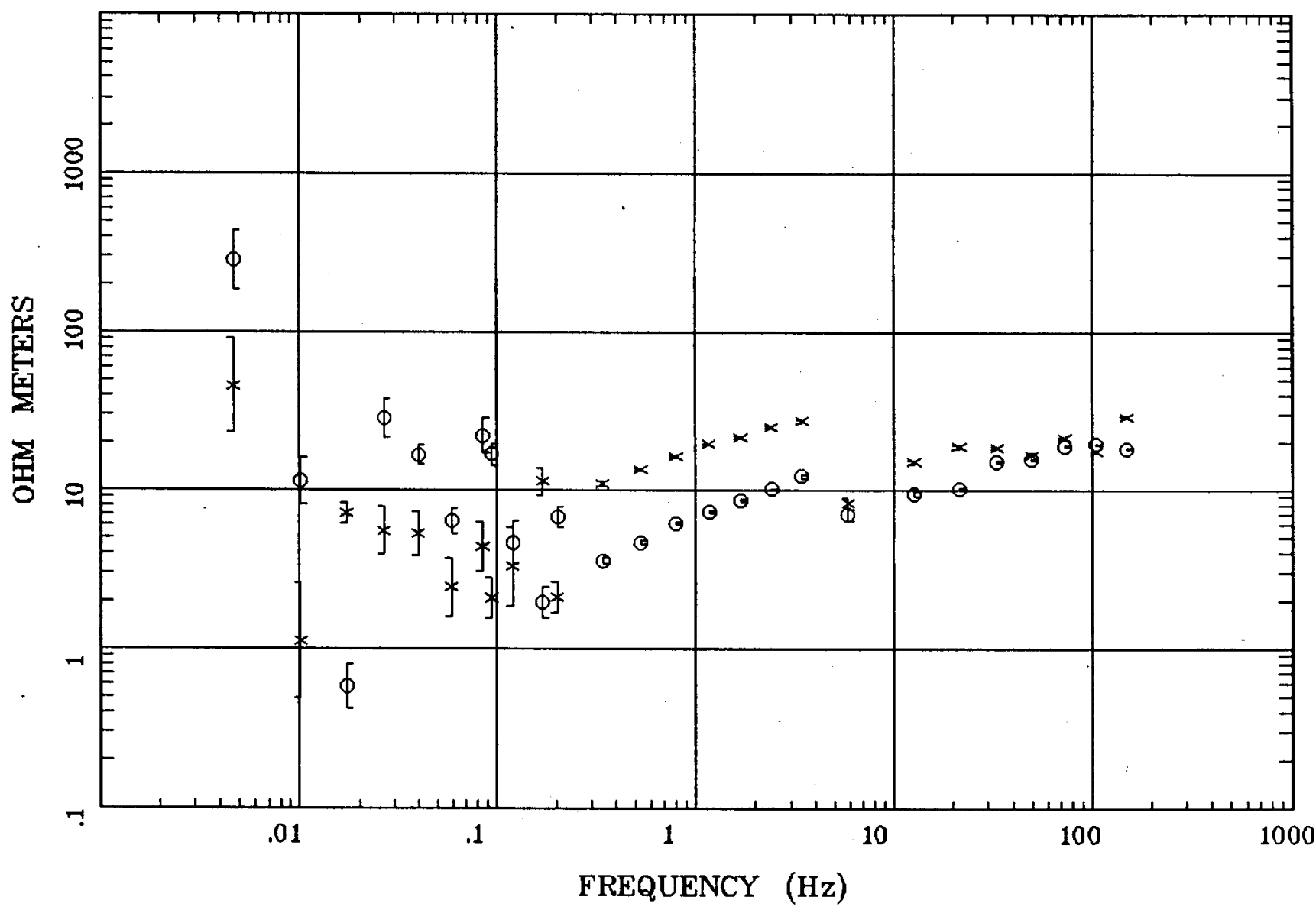

Client:

\section{Remote:}

Acquired:

Survey Co:
Rotation:

Filename: NNR03

Channels: Ch1 Ch2 Ch3 Ch4 Ch5 Ch6 Ch7

Plotted: 15:35 Dec 11, 2000

< EMI - ElectroMagnetic Instruments > 


\section{Station 3}

\section{IMPEDANCE PHASE}

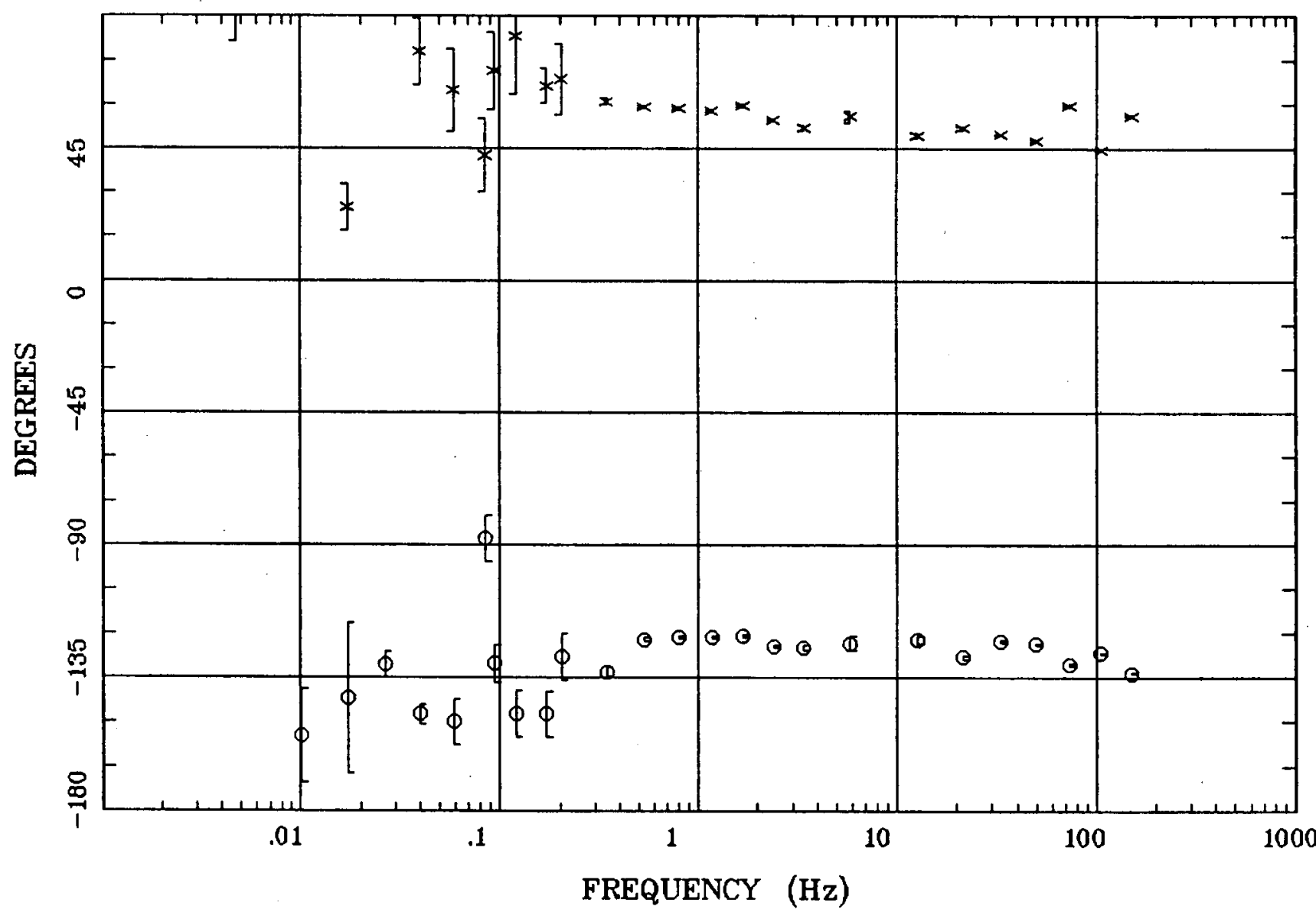

Client:

Remote:

Acquired:

Survey Co:

Rotation:

Filename: NNR03

Channels: Ch1 Ch2 Ch3 Ch4 Ch5 Ch6 Ch7

Plotted: 15:35 Dec 11, 2000

< EMI - ElectroMagnetic Instruments 


\section{Station 3}

\section{ROTATION ANGLE}

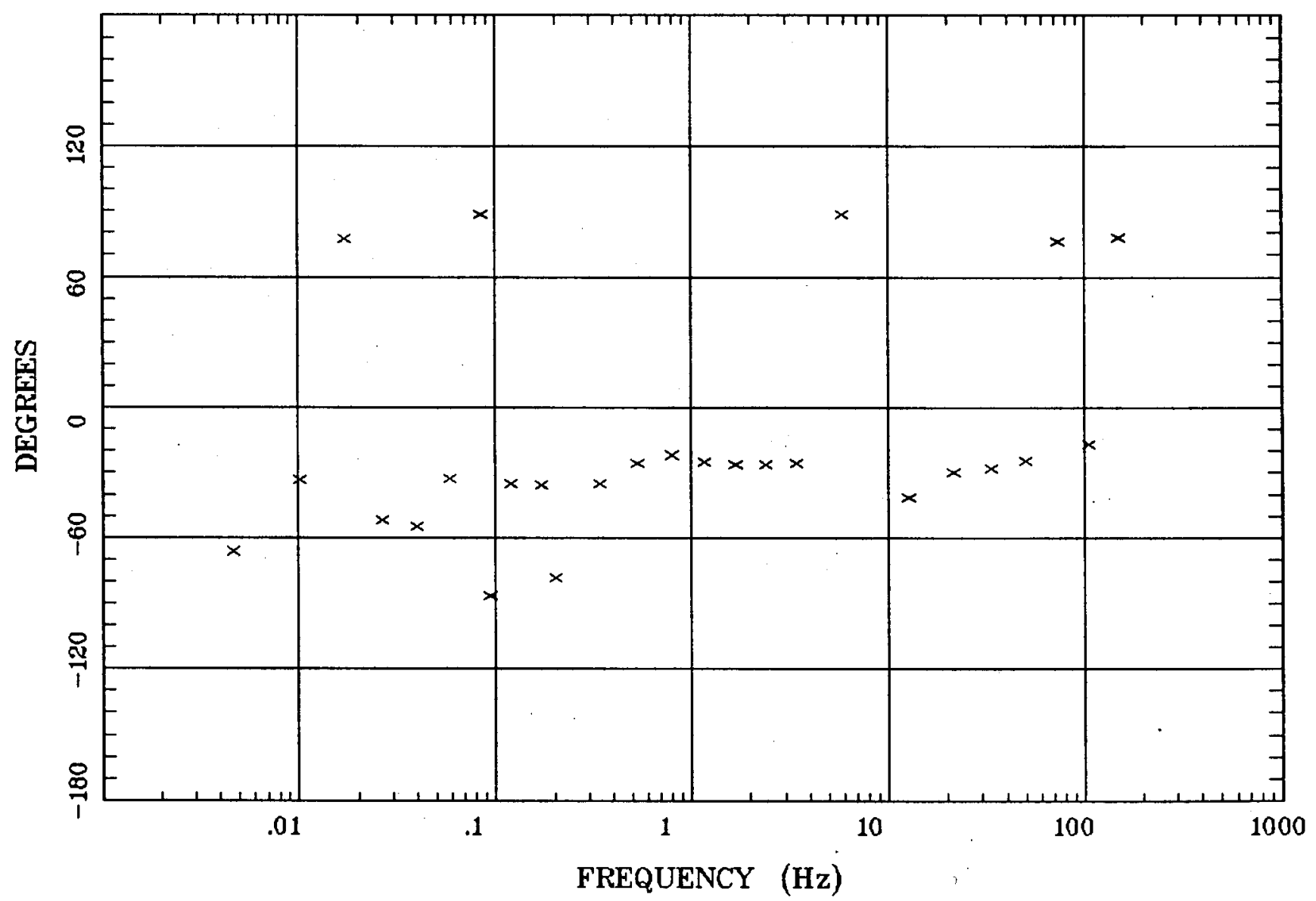

Client:

Remote:

Acquired:

Survey Co:
Rotation:

Filename: NNR03

Channels: Ch1 Ch2 Ch3 Ch4 Ch5 Ch6 Ch7

Plotted: 15:36 Dec 11, 2000

$<$ EMI - ElectroMagnetic Instruments 


\section{IMPEDANCE SKEW}

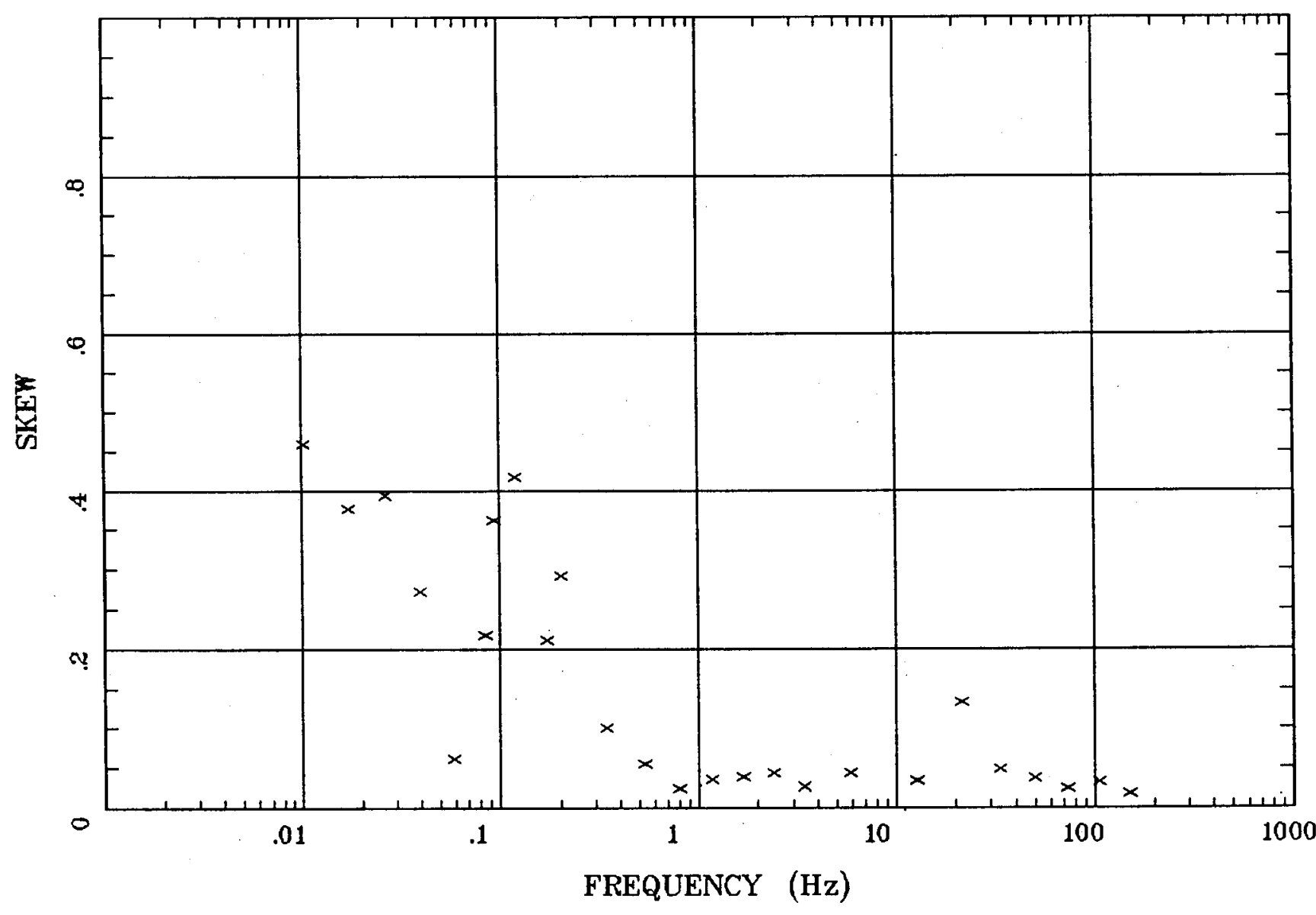

Client:

Remote:

Acquired: Survey Co:

Rotation:

Filename: NNR03

Channels: Ch1 Ch2 Ch3 Ch4 Ch5 Ch6 Ch7 Plotted: 15:36 Dec 11, 2000

< EMI - ElectroMagnetic Instruments 


\section{E MULT Coh.}

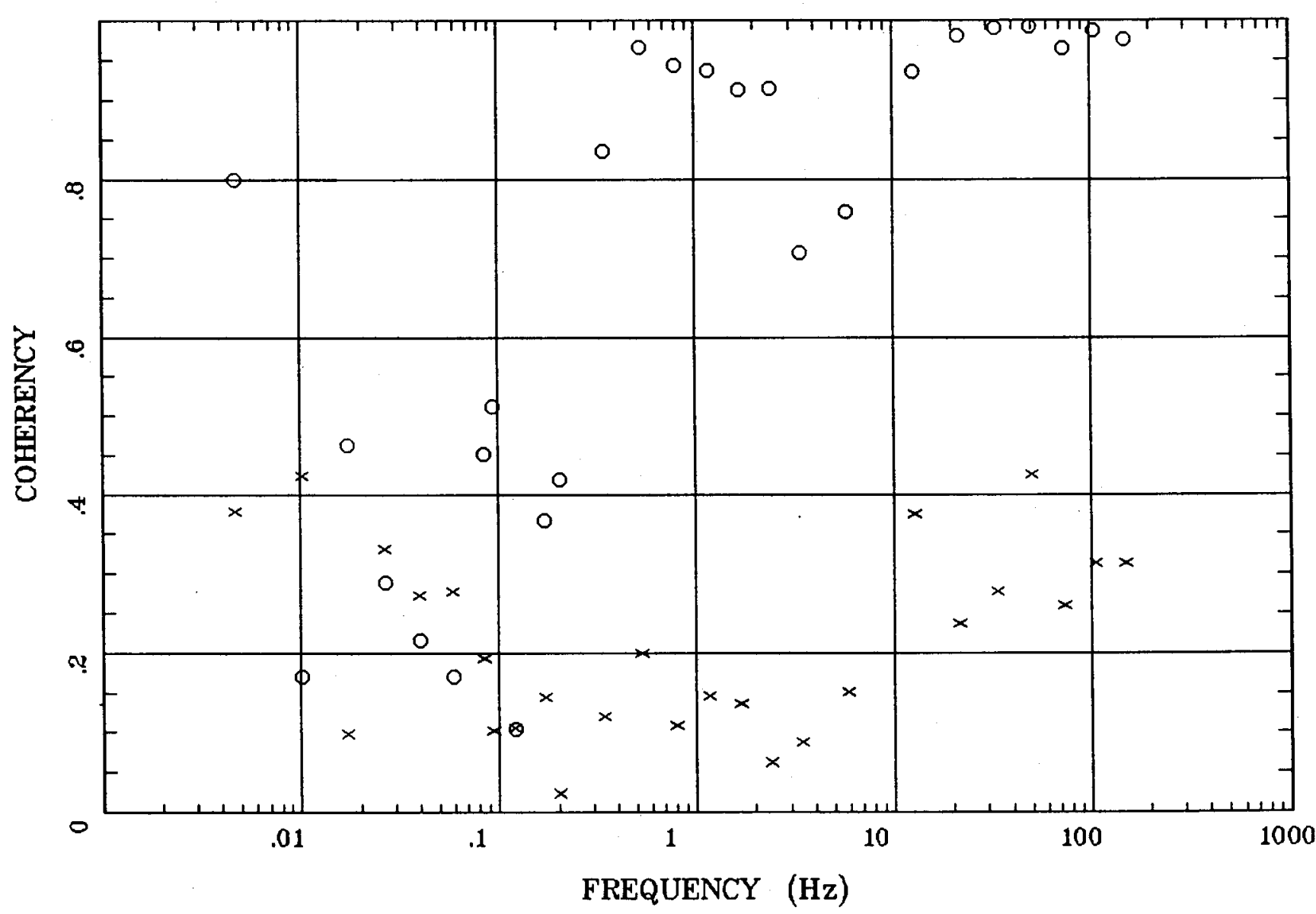

Client:

Remote:

Acquired:

Survey Co:
Rotation:

Filename: NNR03

Channels: Ch1 Ch2 Ch3 Ch4 Ch5 Ch6 Ch7

Plotted: 15:36 Dec 11, 2000

< EMI - ElectroMagnetic Instruments > 


\section{POLAR PLOTS}

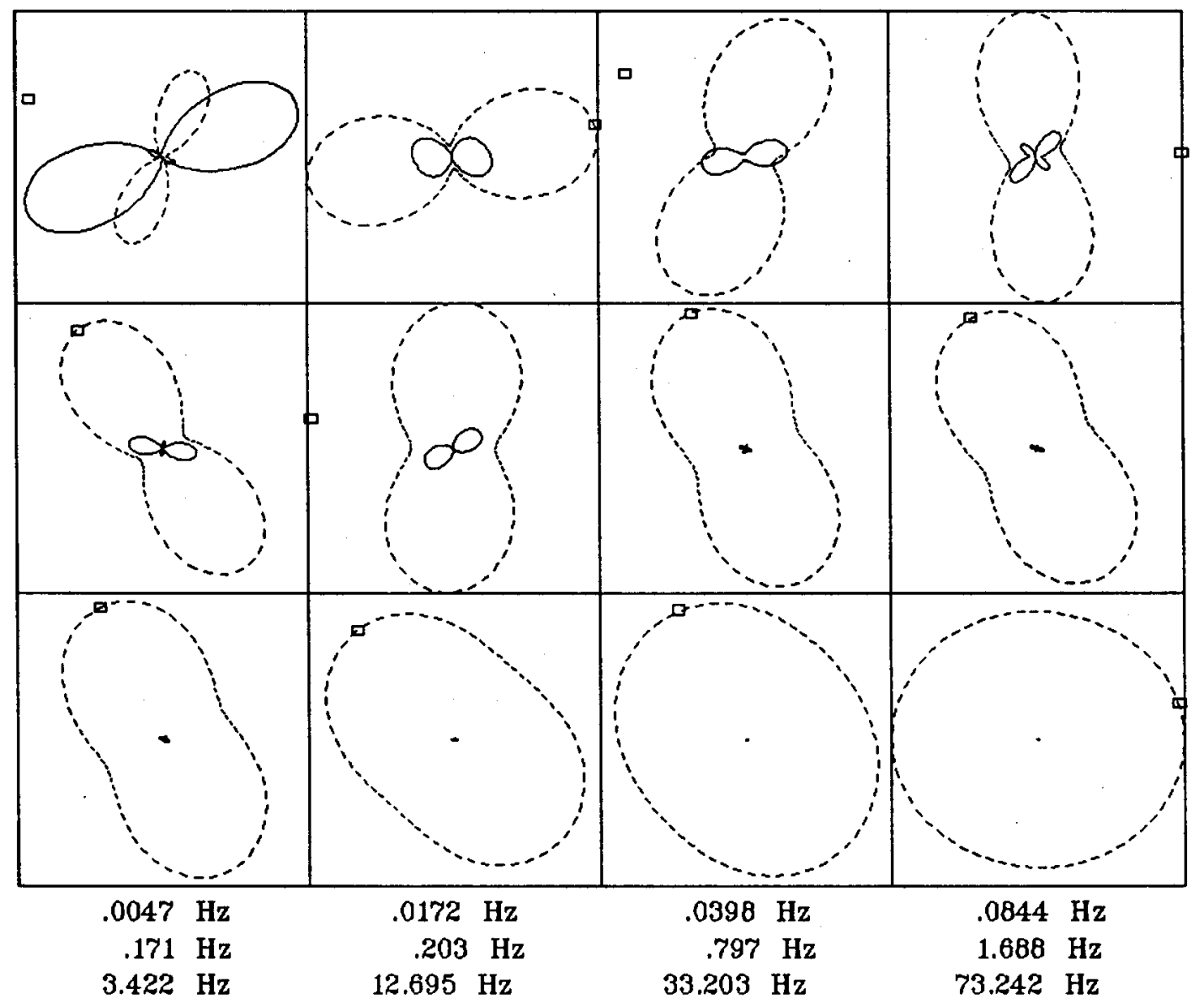

Client:

Remote:

Acquired:

Survey Co:
Rotation:

Filename: NNR03

Channels: Ch1 Ch2 Ch3 Ch4 Ch5 Ch6 Ch7 Plotted: 15:36 Dec 11, 2000

< EMI - ElectroMagnetic Instruments 


\section{Station 4}

\section{APPARENT RESISTIVITY}

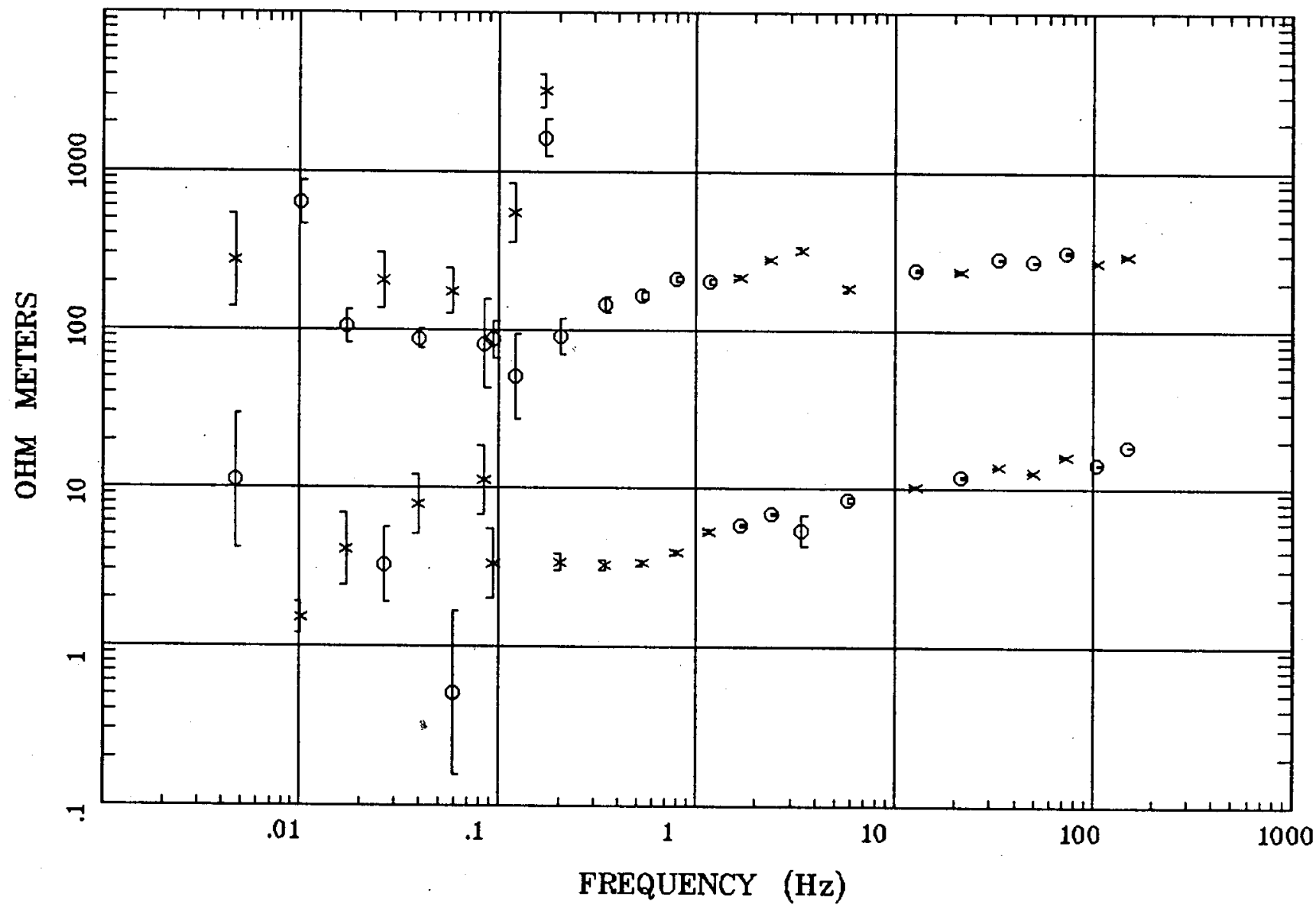

Client:

Remote:

Acquired:

Survey Co:
Rotation:

Filename: NNR04

Channels: Ch1 Ch2 Ch3 Ch4 Ch5 Ch6 Ch7

Plotted: 15:37 Dec 11, 2000

< EMI - ElectroMagnetic Instruments > 


\section{Station 4}

\section{IMPEDANCE PHASE}

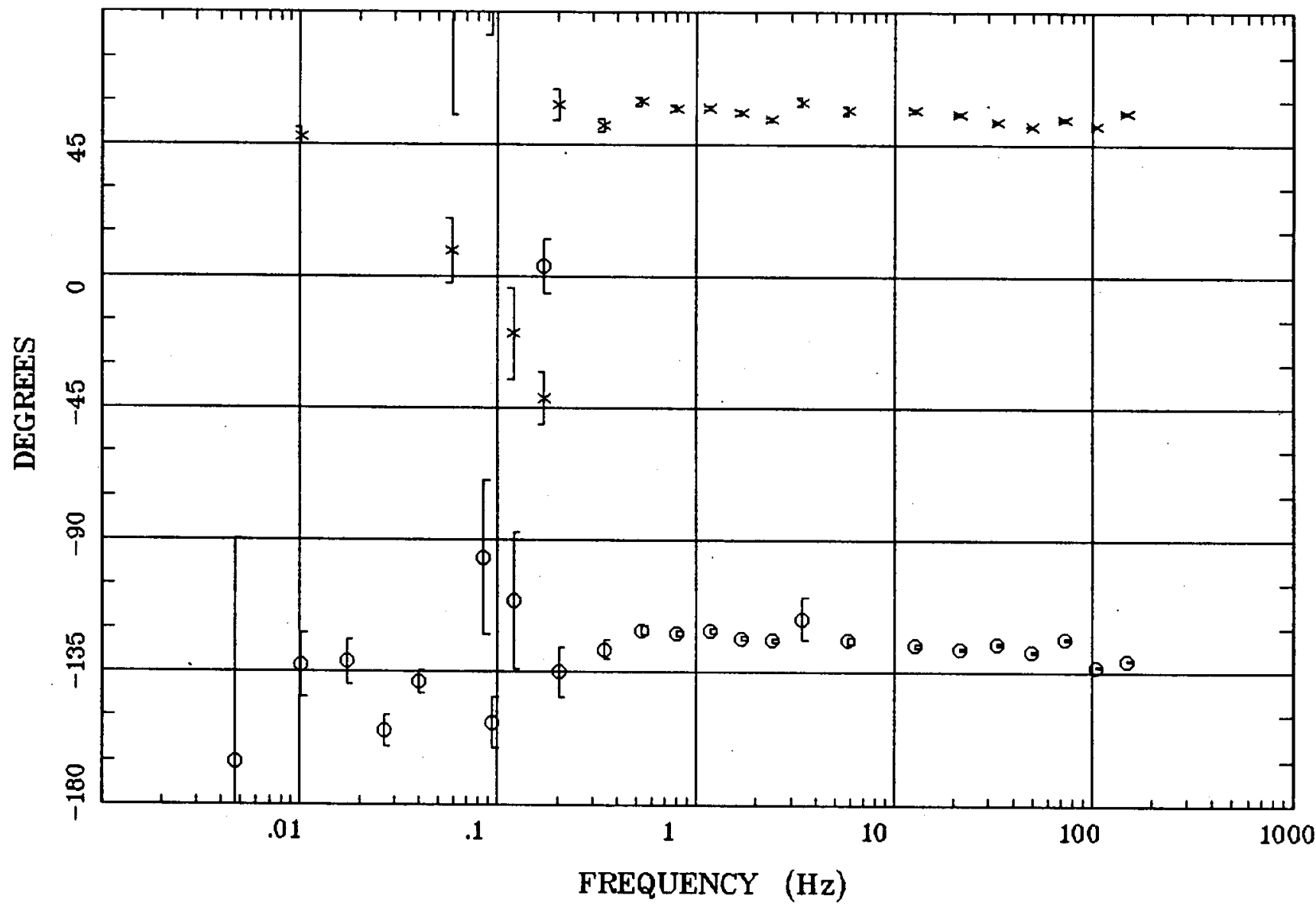

Client:

Remote:

Acquired:

Survey Co:
Rotation:

Filename: NNR04

Channels: Ch1 Ch2 Ch3 Ch4 Ch5 Ch6 Ch7

Plotted: 15:37 Dec 11, 2000

< EMI - ElectroMagnetic Instruments 
Station 4

ROTATION ANGLE

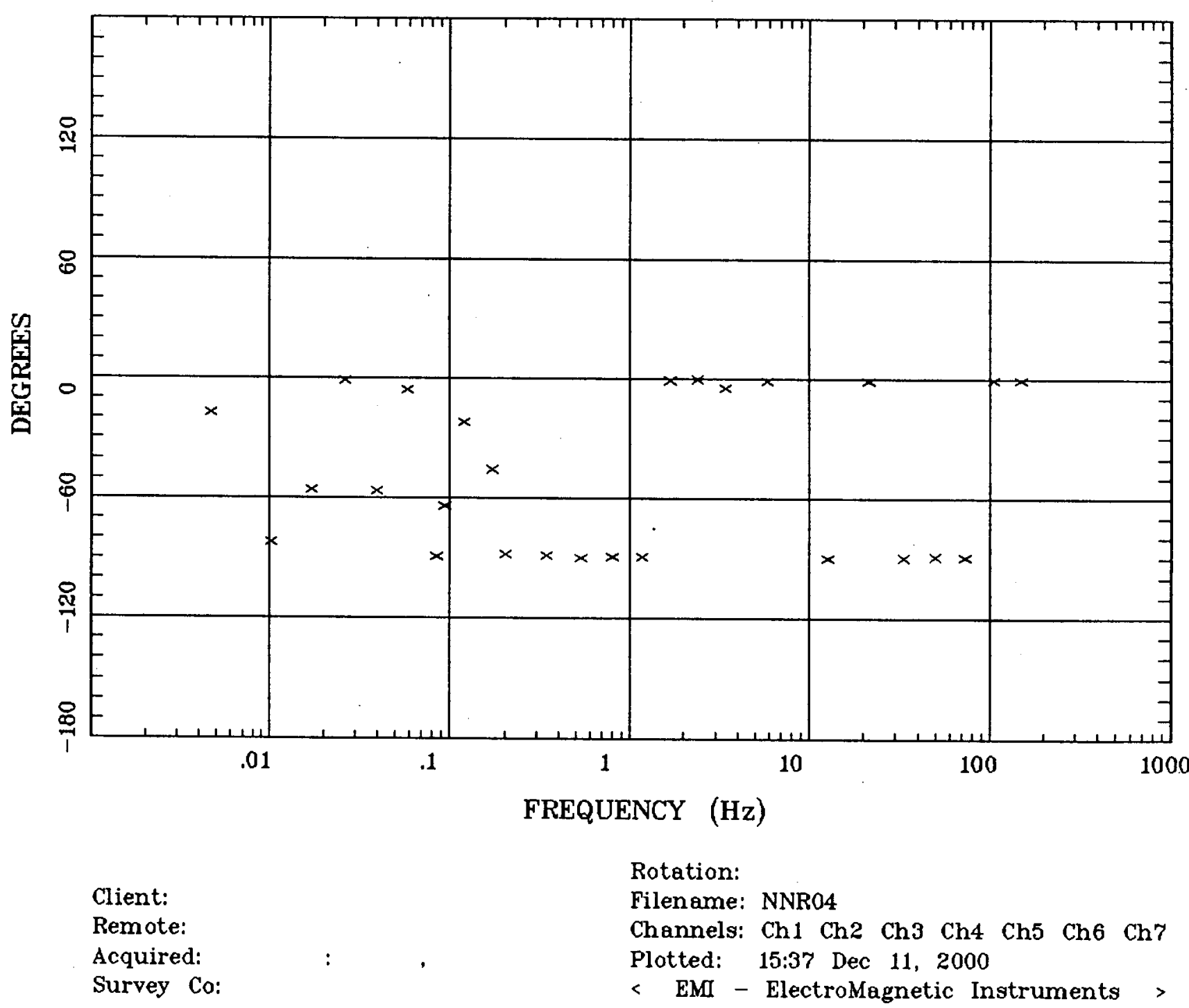


IMPEDANCE SKEW

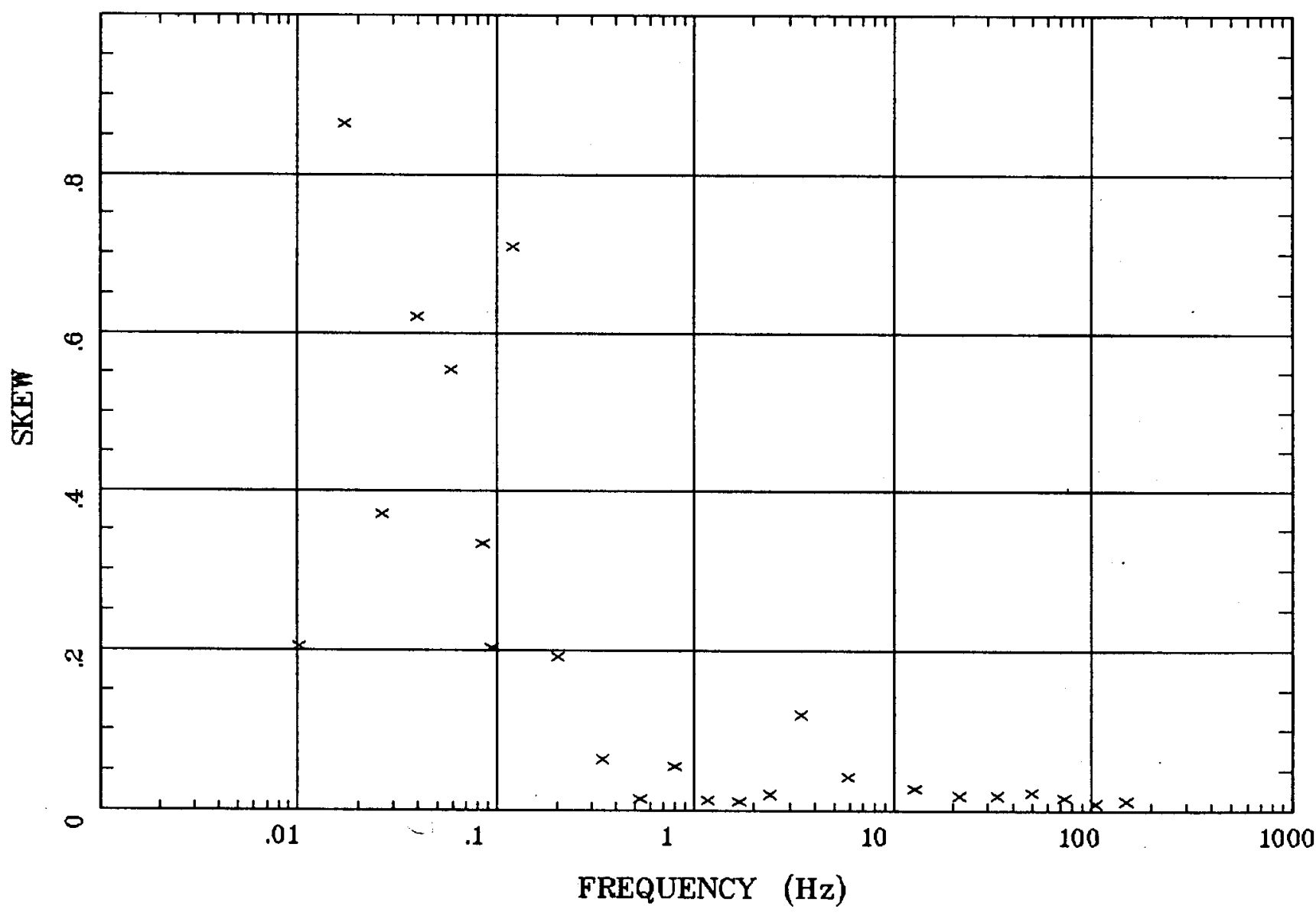

Client:

Remote:

Acquired:

Survey Co:
Rotation:

Filename: NNR04

Channels: Ch1 Ch2 Ch3 Ch4 Ch5 Ch6 Ch7

Plotted: 15:37 Dec 11, 2000

$<$ EMI - ElectroMagnetic Instruments 


\section{Station 4}

E MULT Coh.

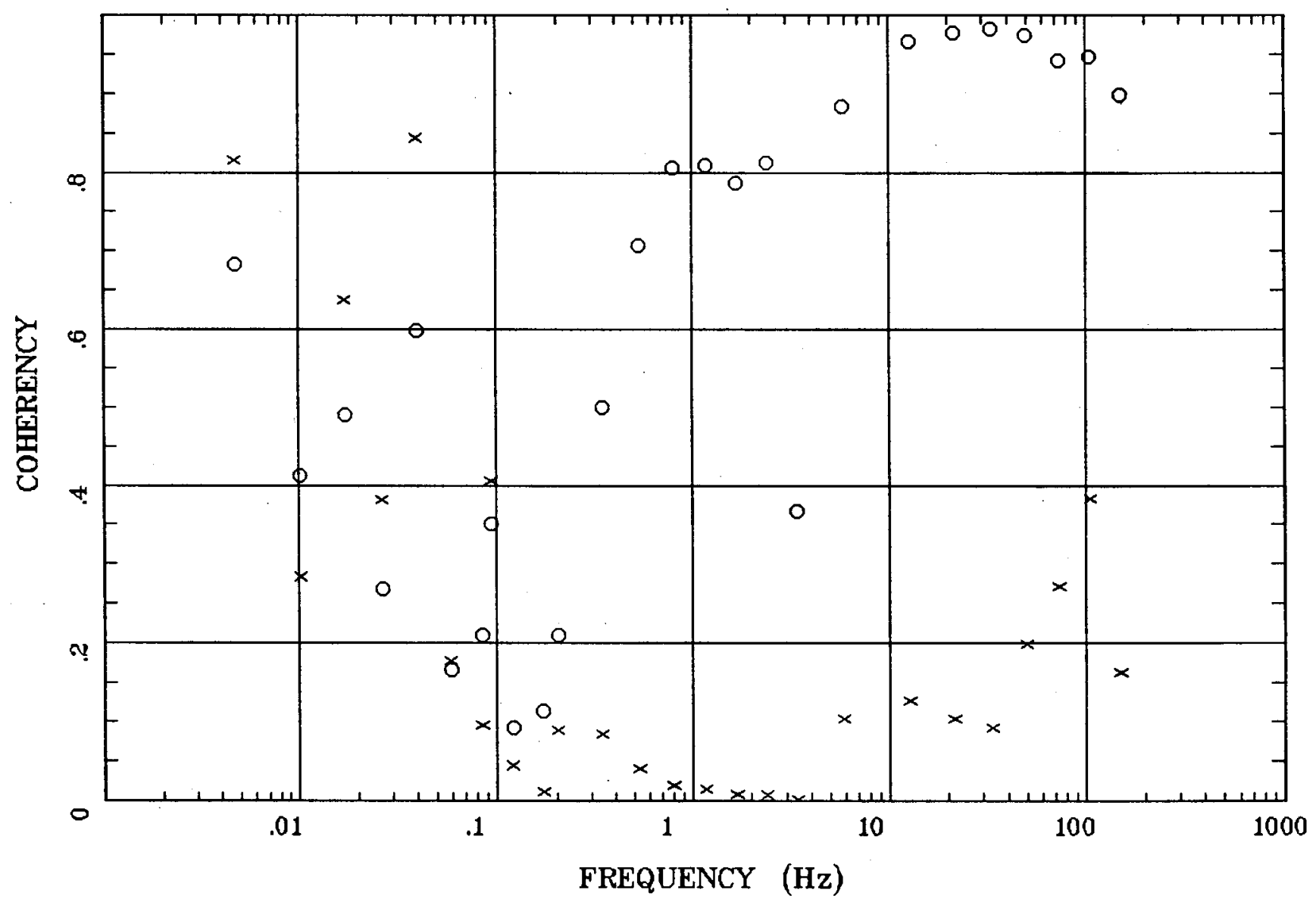

Client:

Remote:

Acquired:

Survey Co:
Rotation:

Filename: NNR04

Channels: Ch1 Ch2 Ch3 Ch4 Ch5 Ch6 Ch7

Plotted: 15:37 Dec 11, 2000

< EMI - ElectroMagnetic Instruments 


\section{POLAR PLOTS}

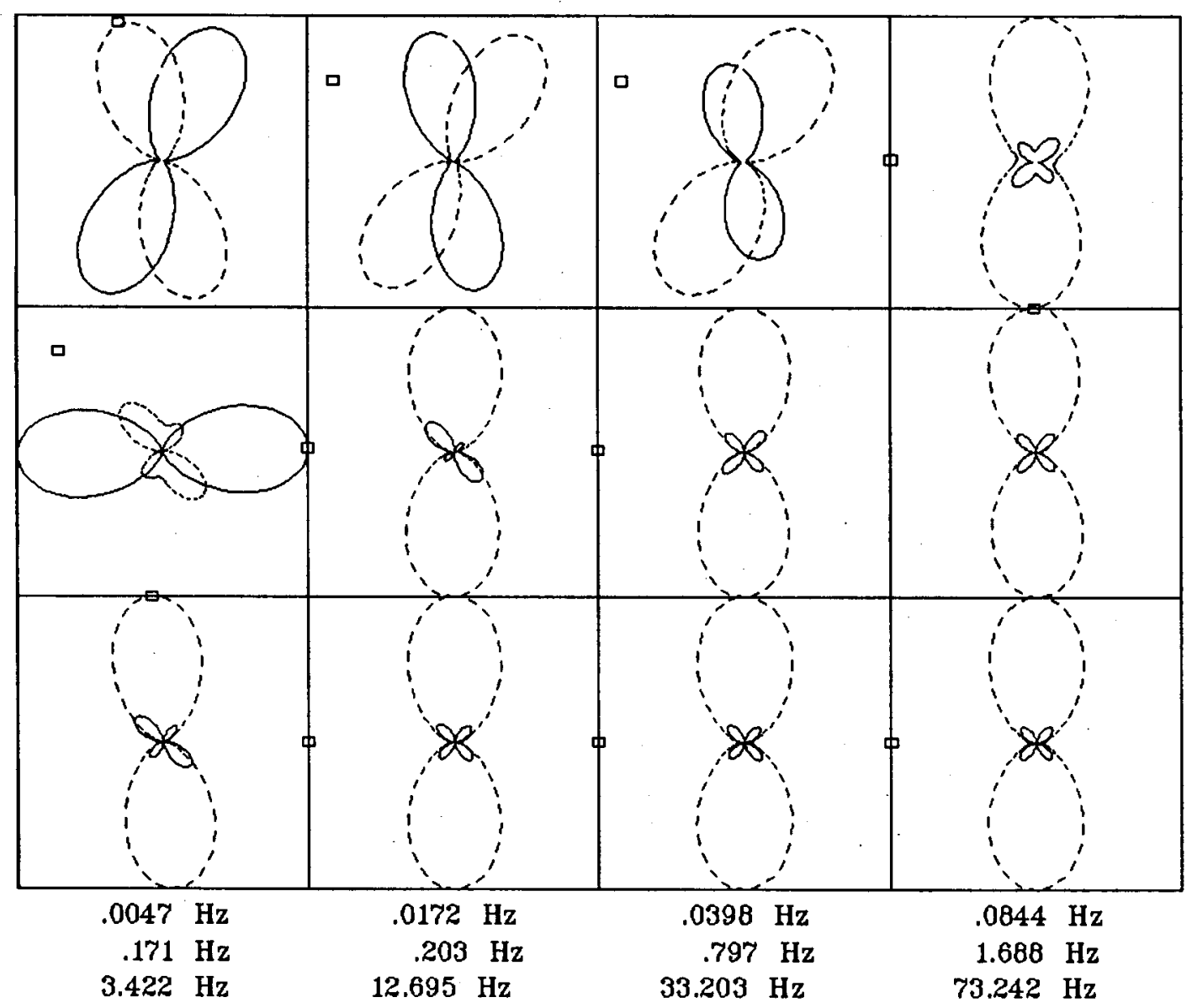

Client:

Remote:

Acquired:

Survey Co:
Rotation:

Filename: NNR04

Channels: Ch1 Ch2 Ch3 Ch4 Ch5 Ch6 Ch7 Plotted: 15:37 Dec 11, 2000

$<$ EMI - ElectroMagnetic Instruments 


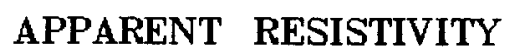

Crescent Valley, NV 100K

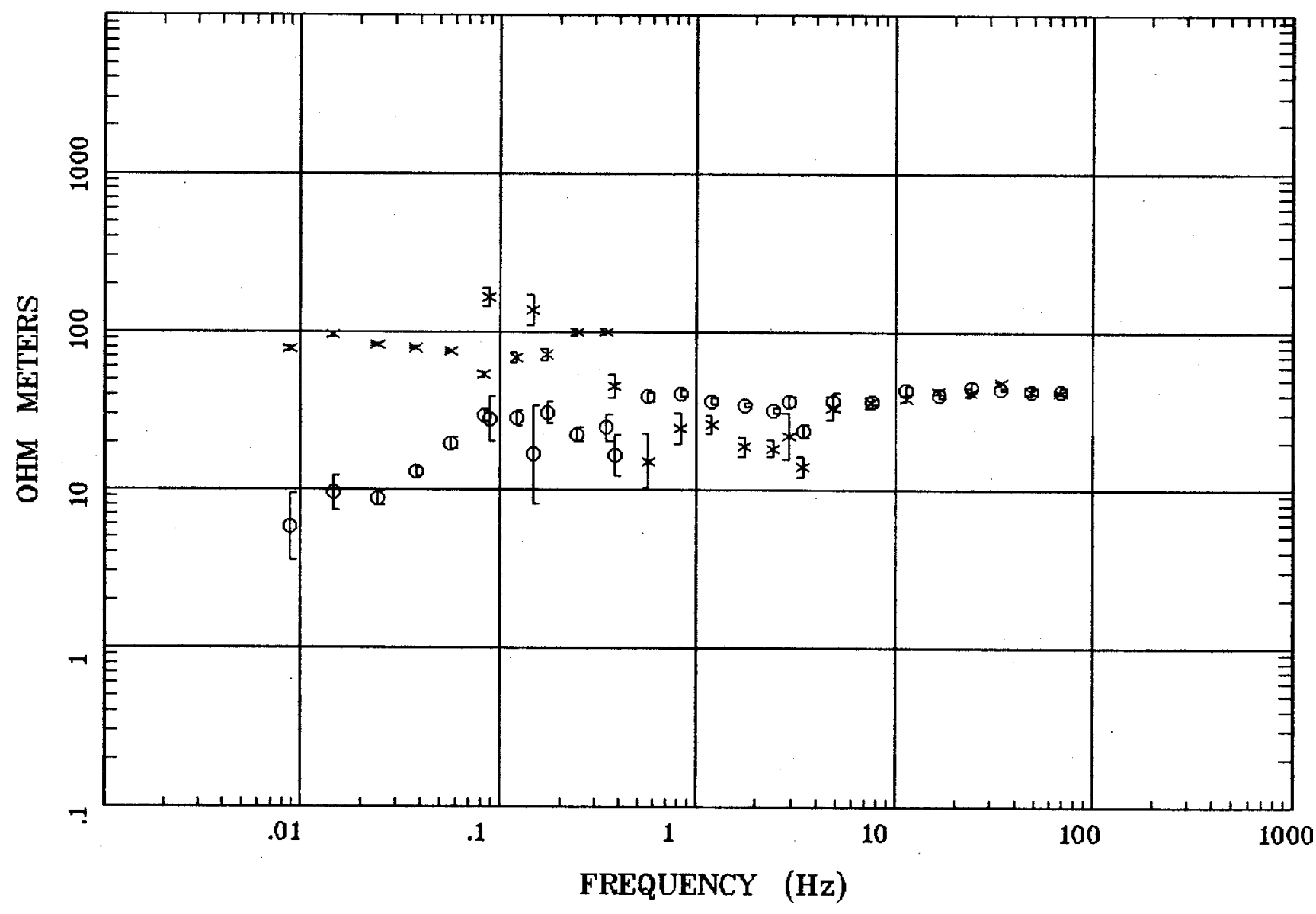

Client:

Remote: none

Acquired: 09:1 Jul 31, 2000

Survey Co:USGS
Rotation:

Filename: hr99a.avg

Channels: Ch1 Ch2 Ch3 Ch4 Ch5 Ch3 Ch4

Plotted: 15:24 Jan 24, 2001

$<$ EMI - ElectroMagnetic Instruments > 


\section{Station 99}

IMPEDANCE PHASE

Crescent Valley, NV 100K

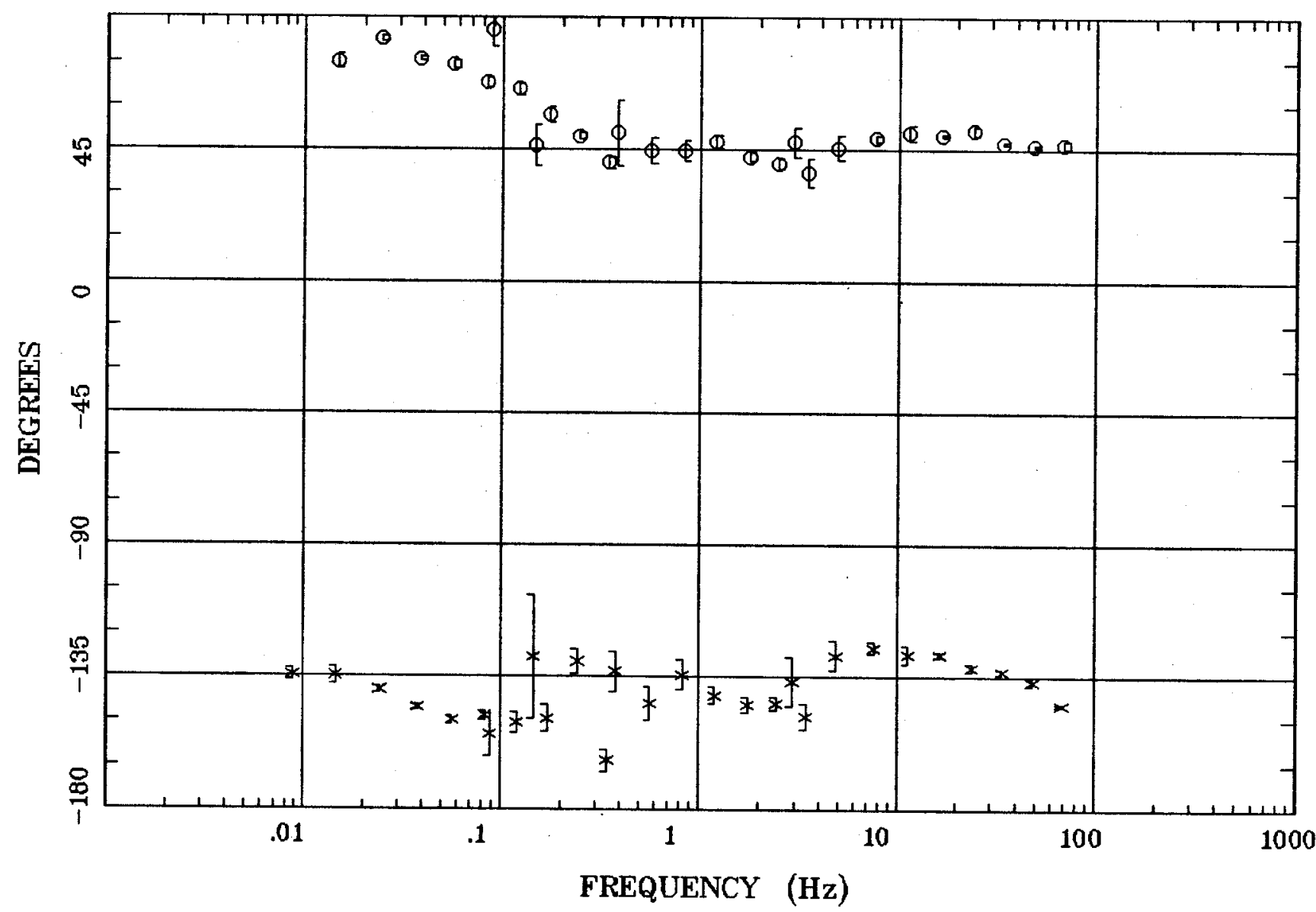

Client:

Remote: none

Acquired: 09:1 Jul 31, 2000 Survey Co:USGS
Rotation:

Filename: hr99a.avg

Channels: Ch1 Ch2 Ch3 Ch4 Ch5 Ch3 Ch4

Plotted: 15:24 Jan 24, 2001

< EMI - ElectroMagnetic Instruments 


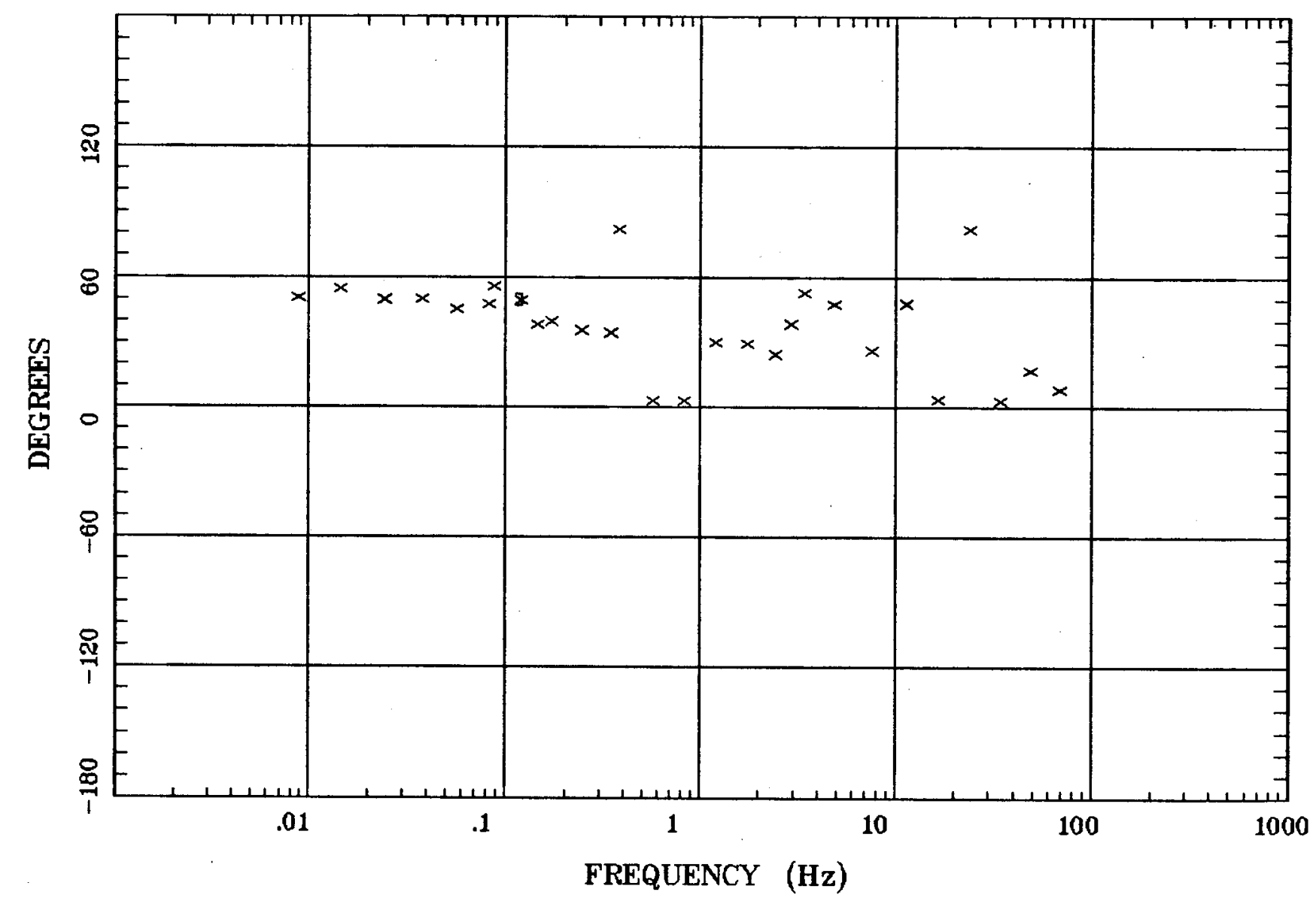

Client:

Remote: none

Acquired: 09:1 Jul 31, 2000

Survey Co:USGS
Rotation:

Filename: hr99a.avg

Channels: Ch1 Ch2 Ch3 Ch4 Ch5 Ch3 Ch4 Plotted: 15:24 Jan 24, 2001

< EMI - ElectroMagnetic Instruments 


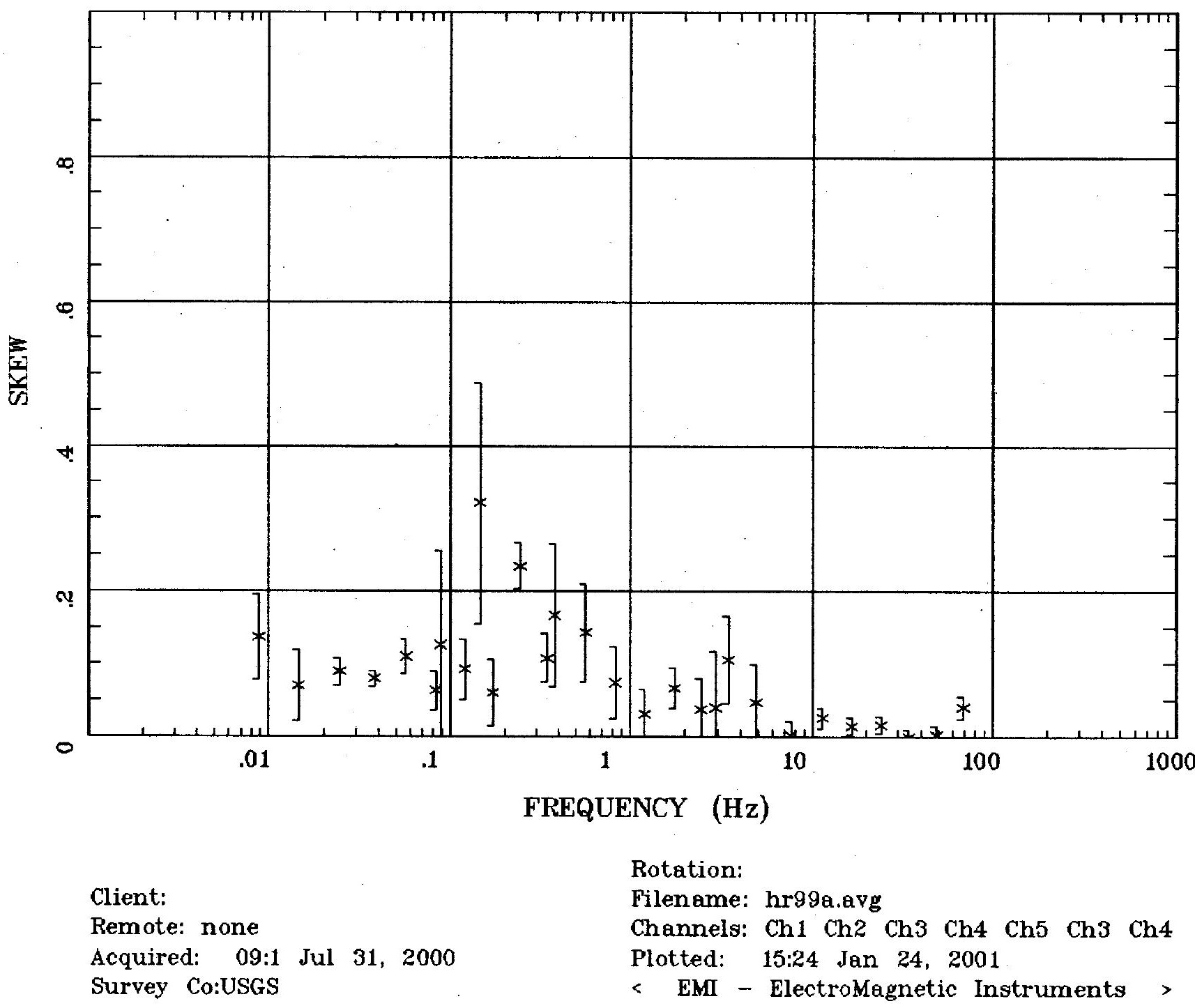


E MULT Coh.

Crescent Valley, NV 100K

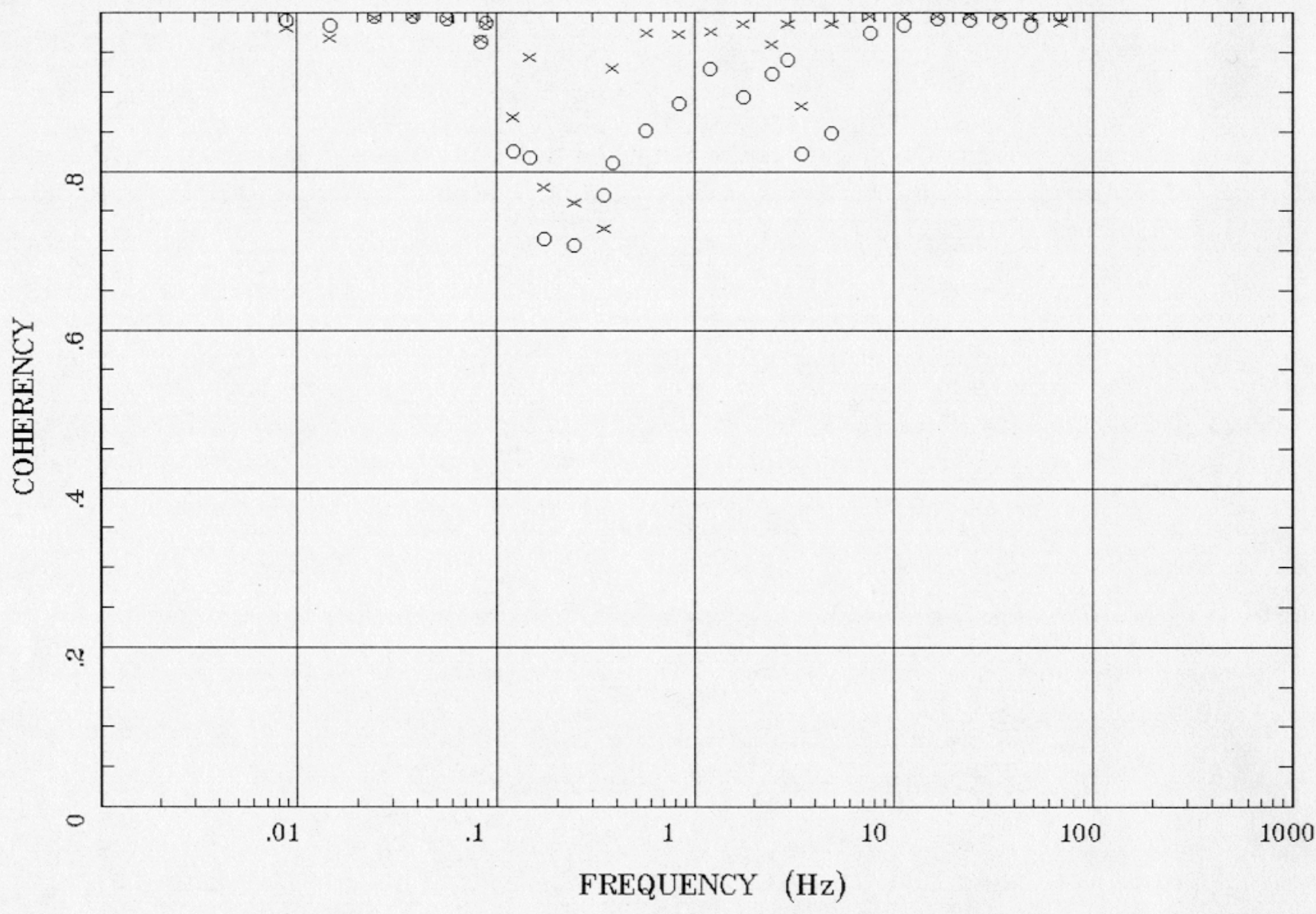

Client:

Remote: none

Acquired: 09:1 Jul 31, 2000

Survey Co:USGS
Rotation:

Filename: hr99a.avg

Channels: Ch1 Ch2 Ch3 Ch4 Ch5 Ch3 Ch4

Plotted: 15:24 Jan 24, 2001

< EMI - ElectroMagnetic Instruments 
Station 99

POLAR PLOTS

Crescent Valley, NV 100K

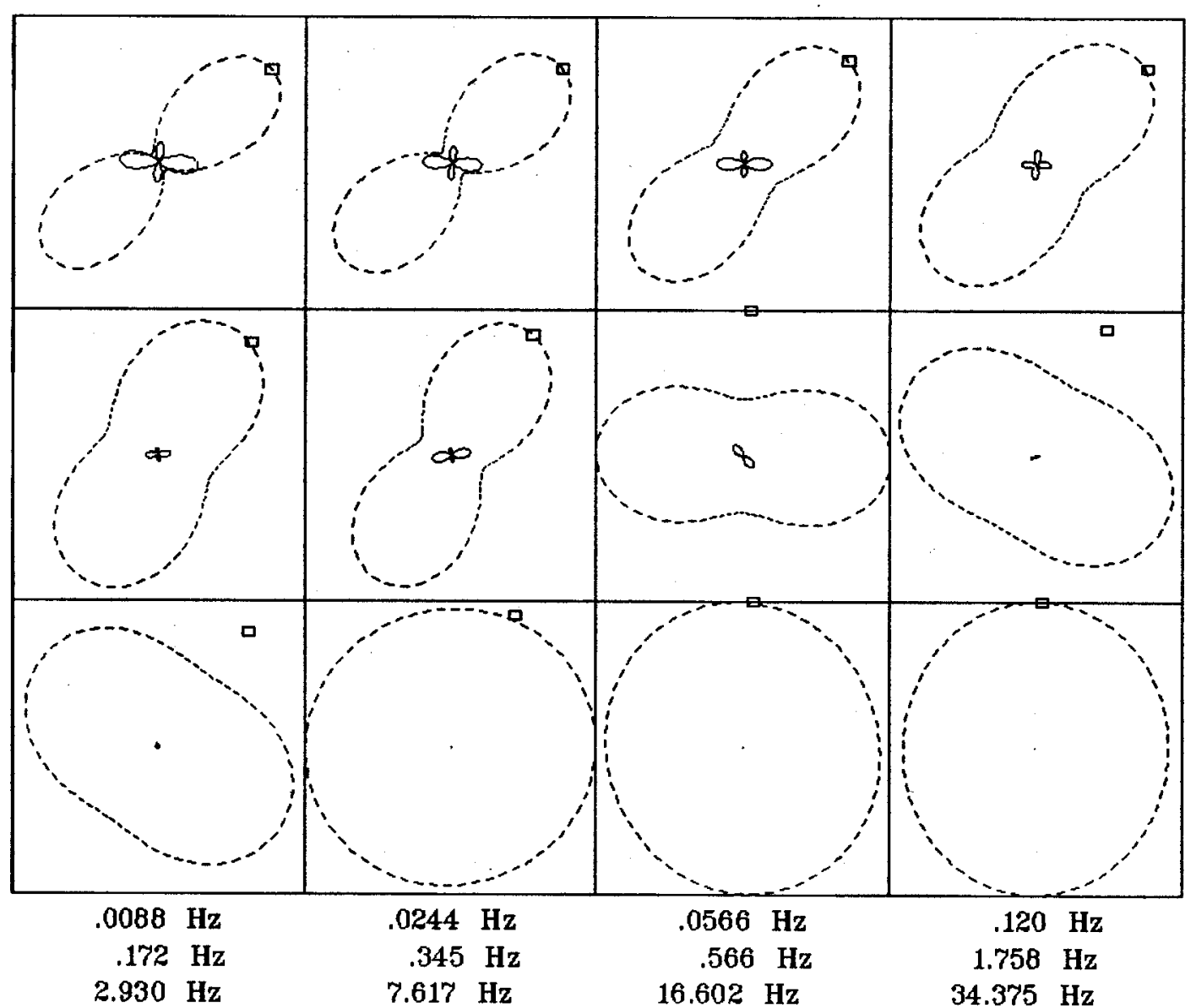

Client:

Rotation:

Remote: none

Filename: hr99a.avg

Acquired: 09:1 Jul 31, 2000

Channels: Ch1 Ch2 Ch3 ch4 Ch5 Ch3 Ch4

Plotted: 15:24 Jan 24, 2001

Survey Co:USGS

< EMI - ElectroMagnetic Instruments > 


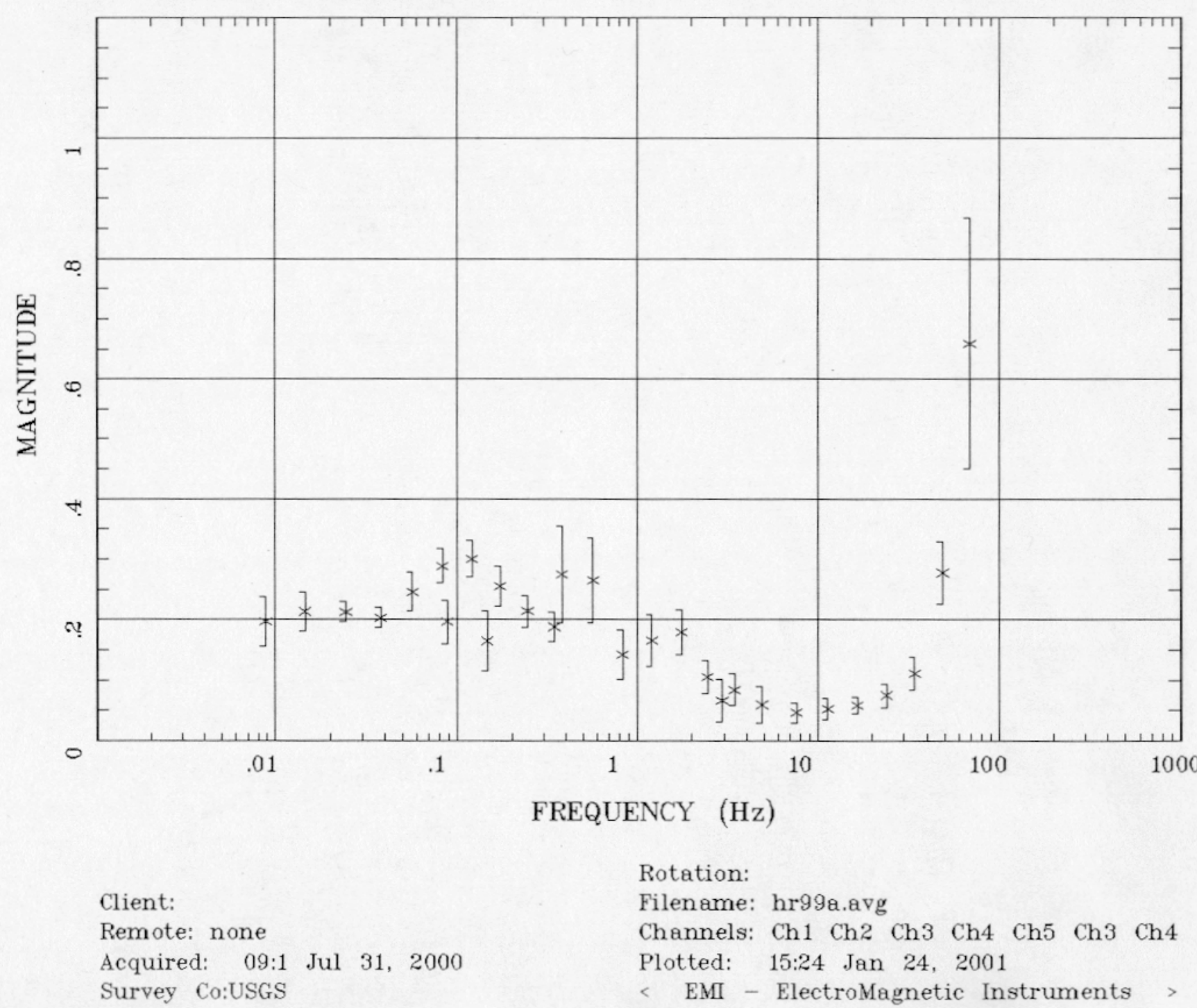


TIPPER STRIKE

Crescent Valley, NV 100K

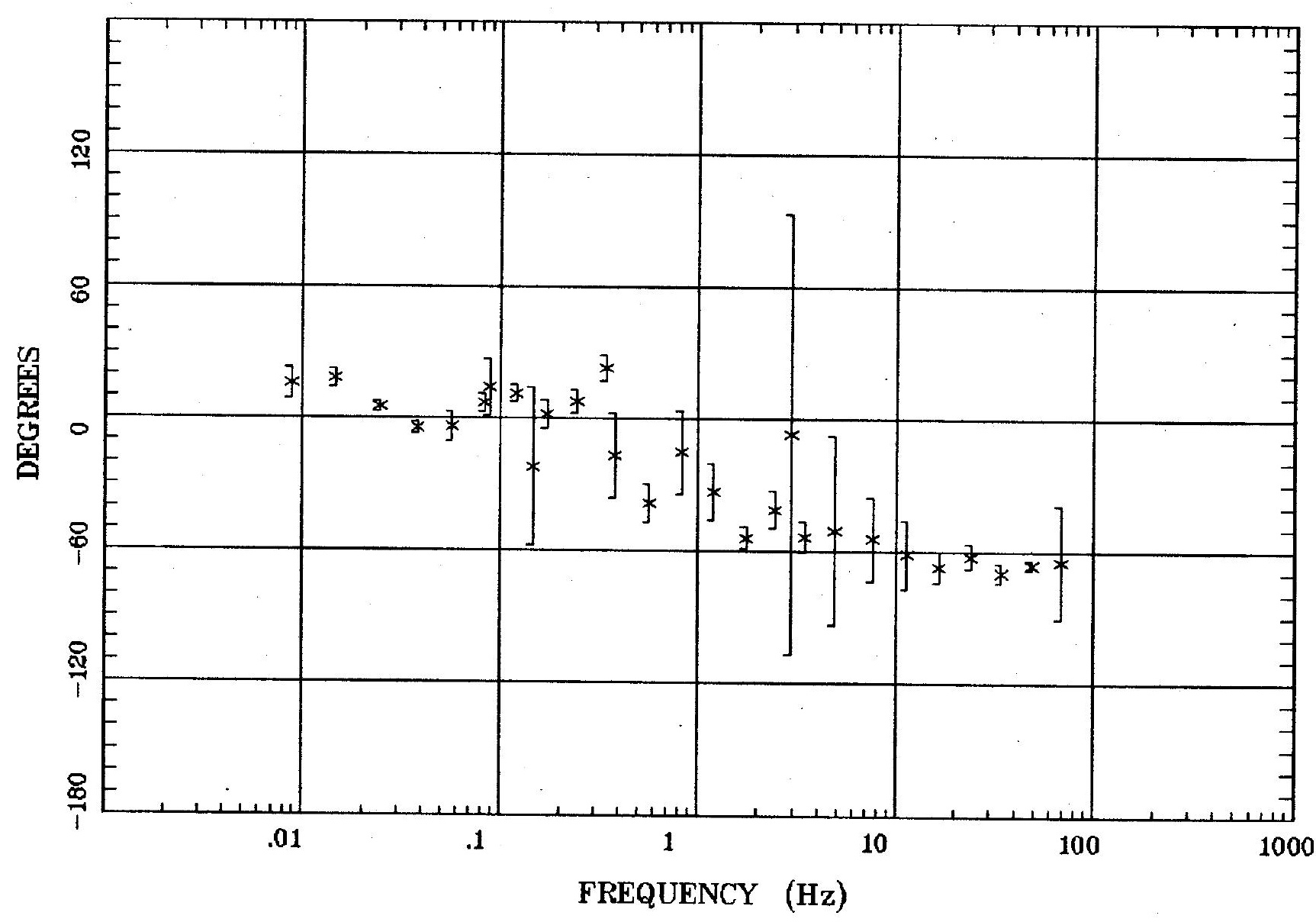

Client:

Remote: none

Acquired: 09:1 Jul 31, 2000

Survey Co:USGS
Rotation:

Filename: hr99a.avg

Channels: Ch1 Ch2 Ch3 Ch4 Ch5 Ch3 Ch4

Plotted: 15:24 Jan 24, 2001

< EMI - ElectroMagnetic Instruments > 


\section{HzHx.x Coh HzHy.o}

Crescent Valley, NV 100K

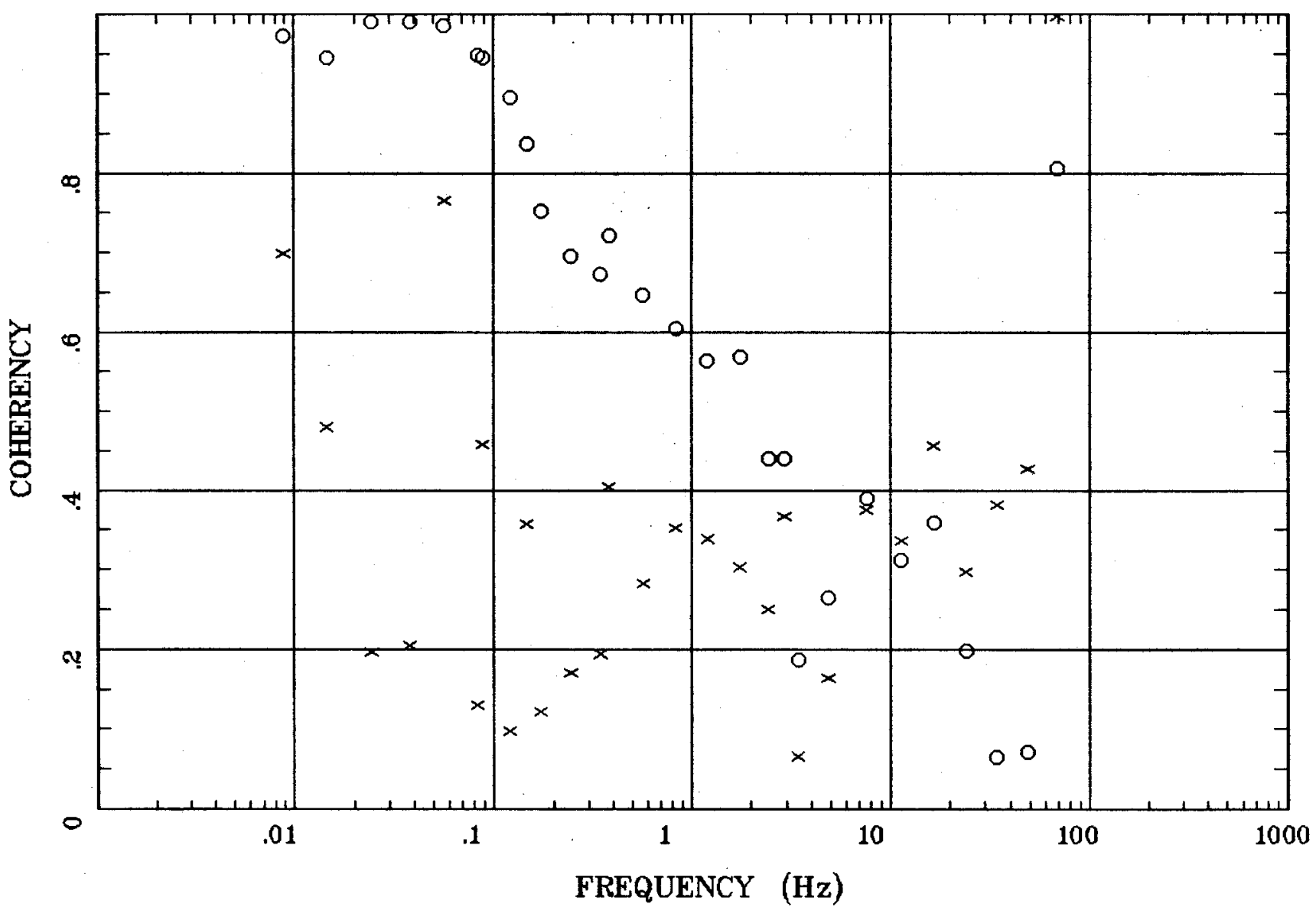

Client:

Remote: none

Acquired: 09:1 Jul 31, 2000

Survey Co:USGS
Rotation:

Filename: hr99a.avg

Channels: Ch1 Ch2 Ch3 Ch4 Ch5 Ch3 Ch4

Plotted: 15:25 Jan 24, 2001

< EMI - ElectroMagnetic Instruments 


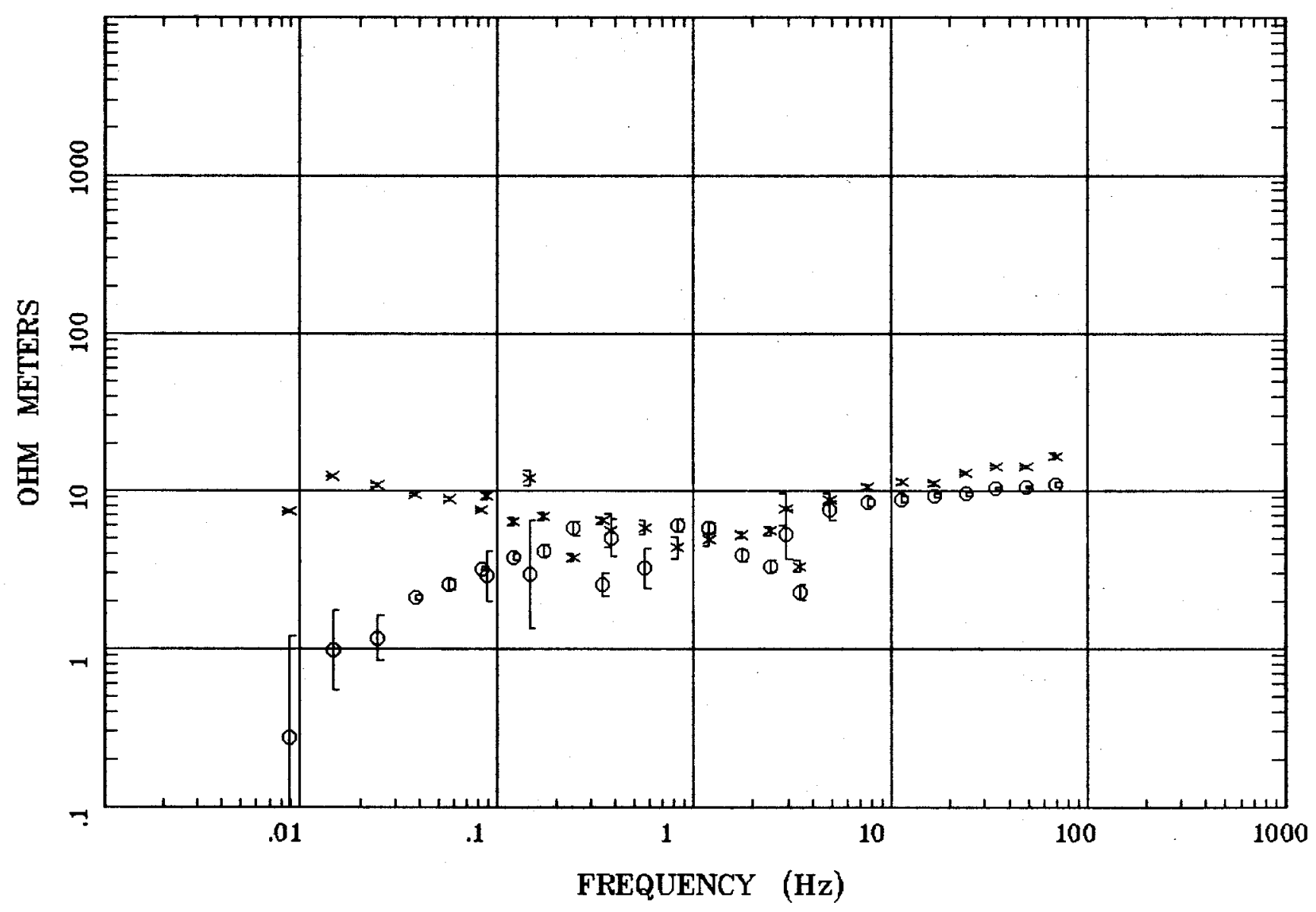

Client:

Remote: none

Acquired: 12:1 Jul 31, 2000 Survey Co:USGS
Rotation:

Filename: hr100a.avg

Channels: Ch1 Ch2 Ch3 Ch4 Ch5 Ch3 Ch4

Plotted: 15:25 Jan 24, 2001

< EMI - ElectroMagnetic Instruments > 
Station 100

IMPEDANCE PHASE

Crescent Valley, NV $100 \mathrm{~K}$

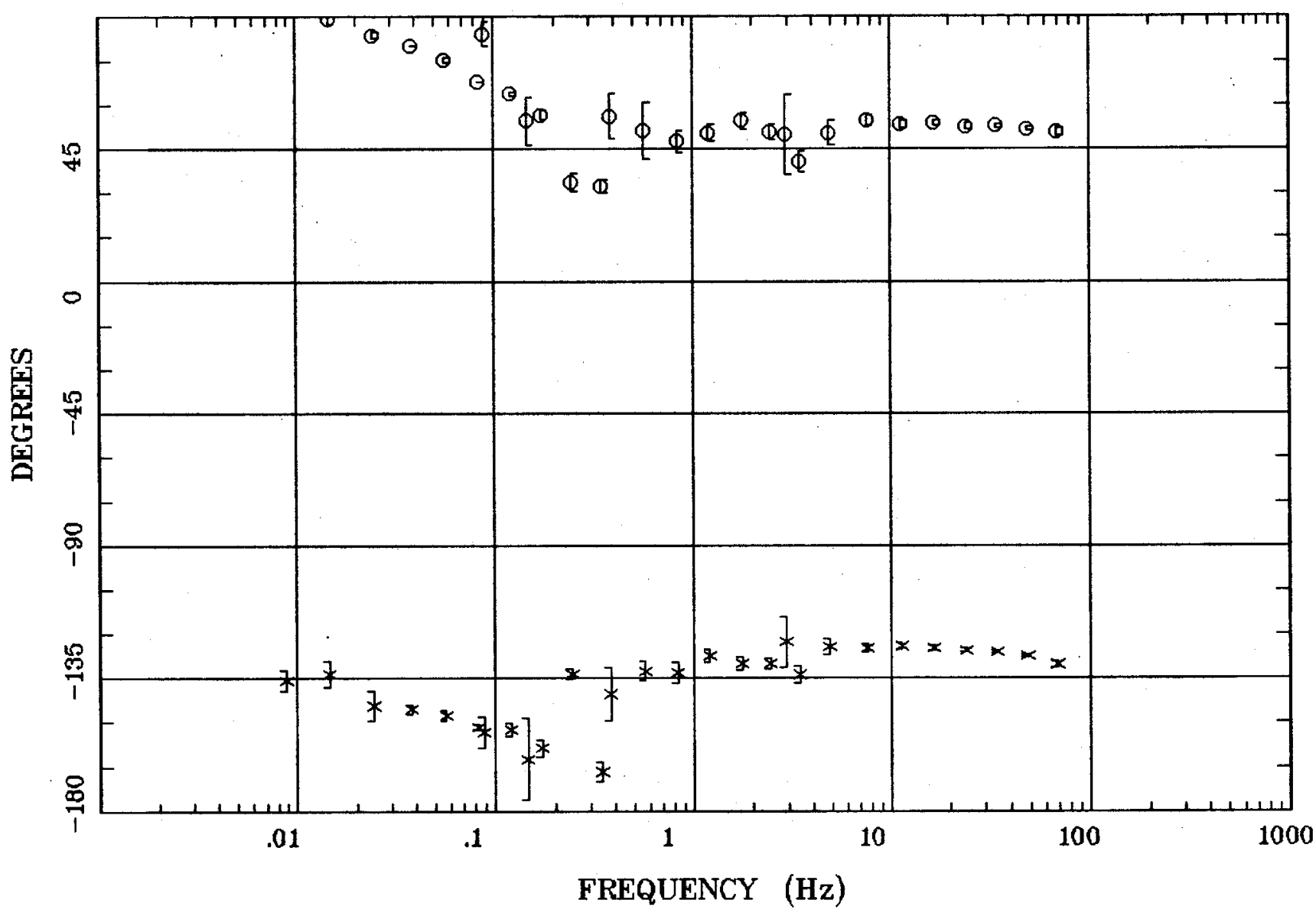

Rotation:

Client:

Filename: hr100a.avg

Remote: none

Acquired: 12:1 Jul 31, 2000

Channels: Ch1 Ch2 Ch3 Ch4 Ch5 Ch3 Ch4

Plotted: 15:25 Jan 24, 2001

Survey Co:USGS

< EMI - ElectroMagnetic Instruments > 


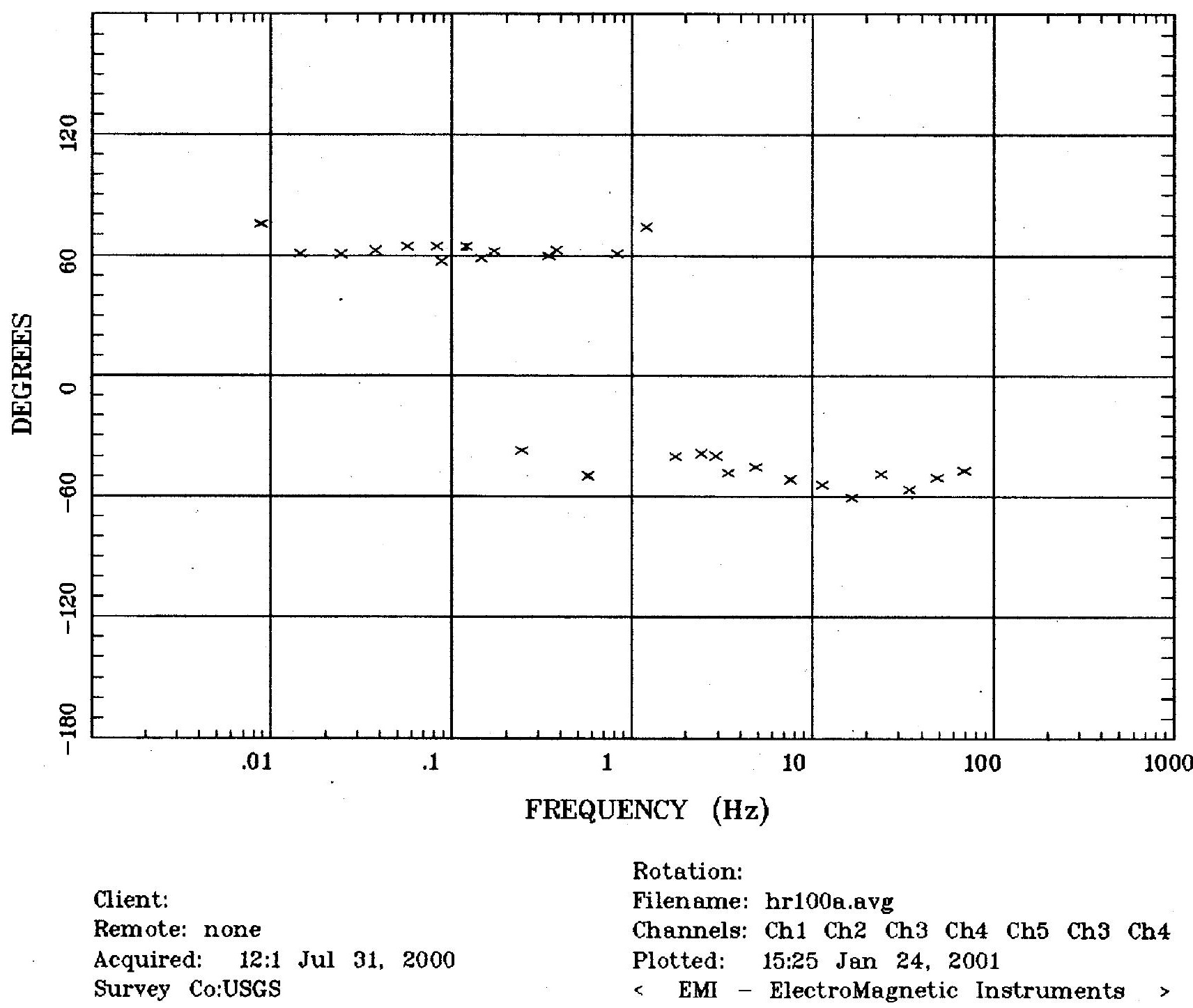




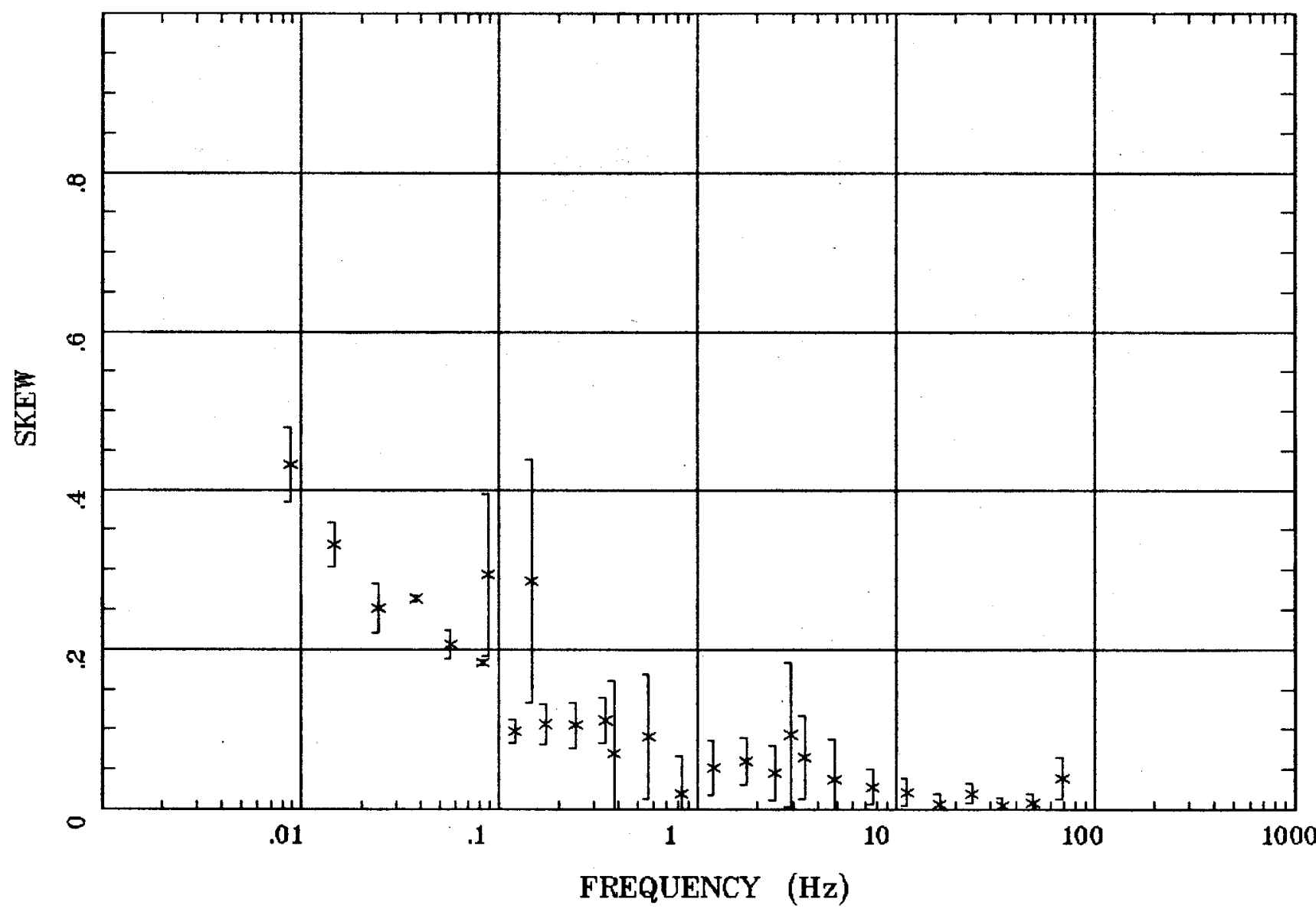

Client:

Remote: none

Acquired: 12:1 Jul 31, 2000 Survey Co:USGS
Rotation:

Filename: hr100a.avg

Channels: Ch1 Ch2 Ch3 Ch4 Ch5 Ch3 Ch4 Plotted: 15:25 Jan 24, 2001

$<$ EMI - ElectroMagnetic Instruments 
E MULT Coh.

Crescent Valley, NV 100K

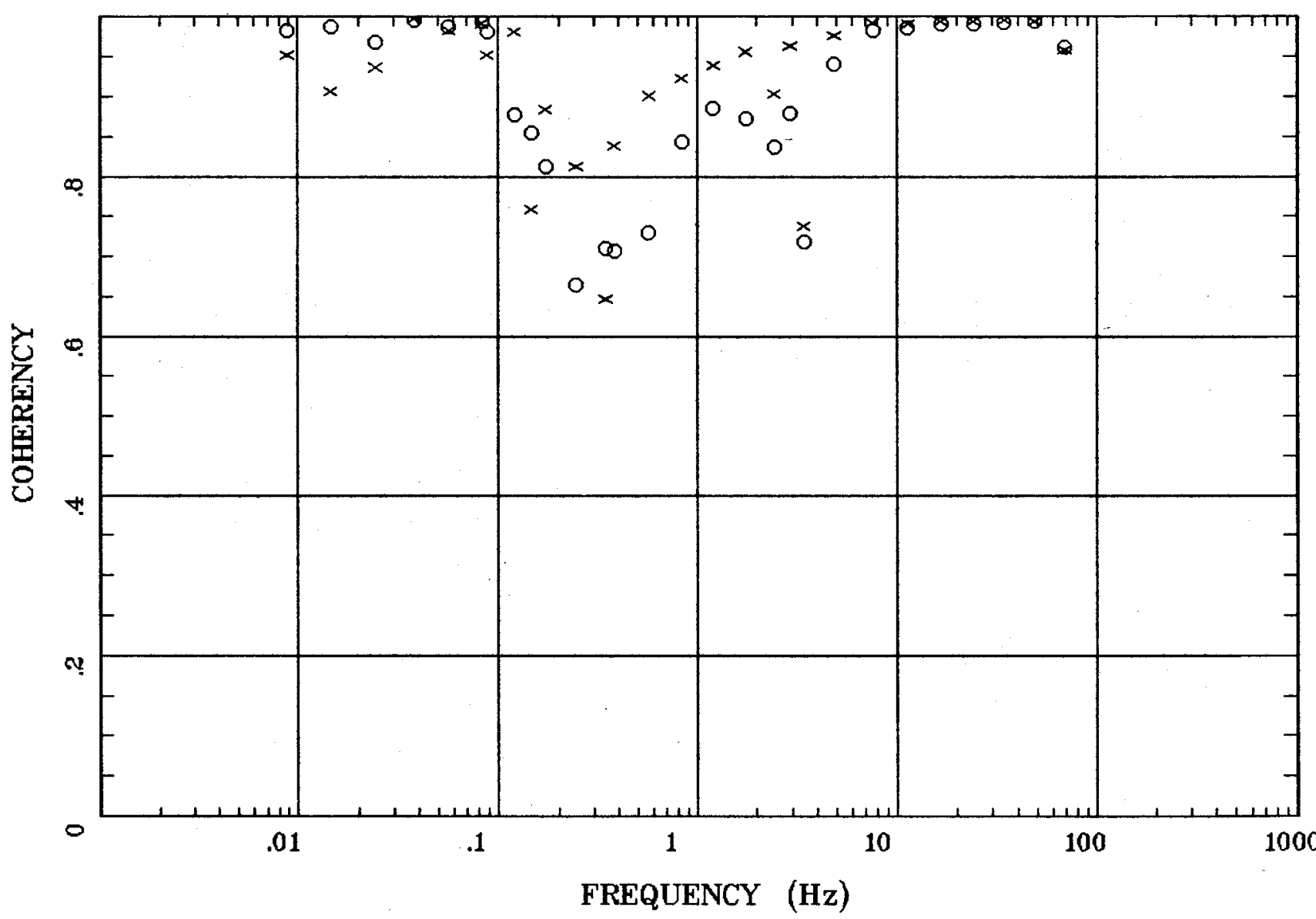

Client:

Remote: none

Acquired: 12:1 Jul 31, 2000

Survey Co:USGS
Rotation:

Filename: hr100a.avg

Channels: Ch1 Ch2 Ch3 Ch4 Ch5 Ch3 Ch4

Plotted: 15:25 Jan 24, 2001

$<$ EMI - ElectroMagnetic Instruments 


\section{POLAR PLOTS}

Crescent Valley, NV 100K

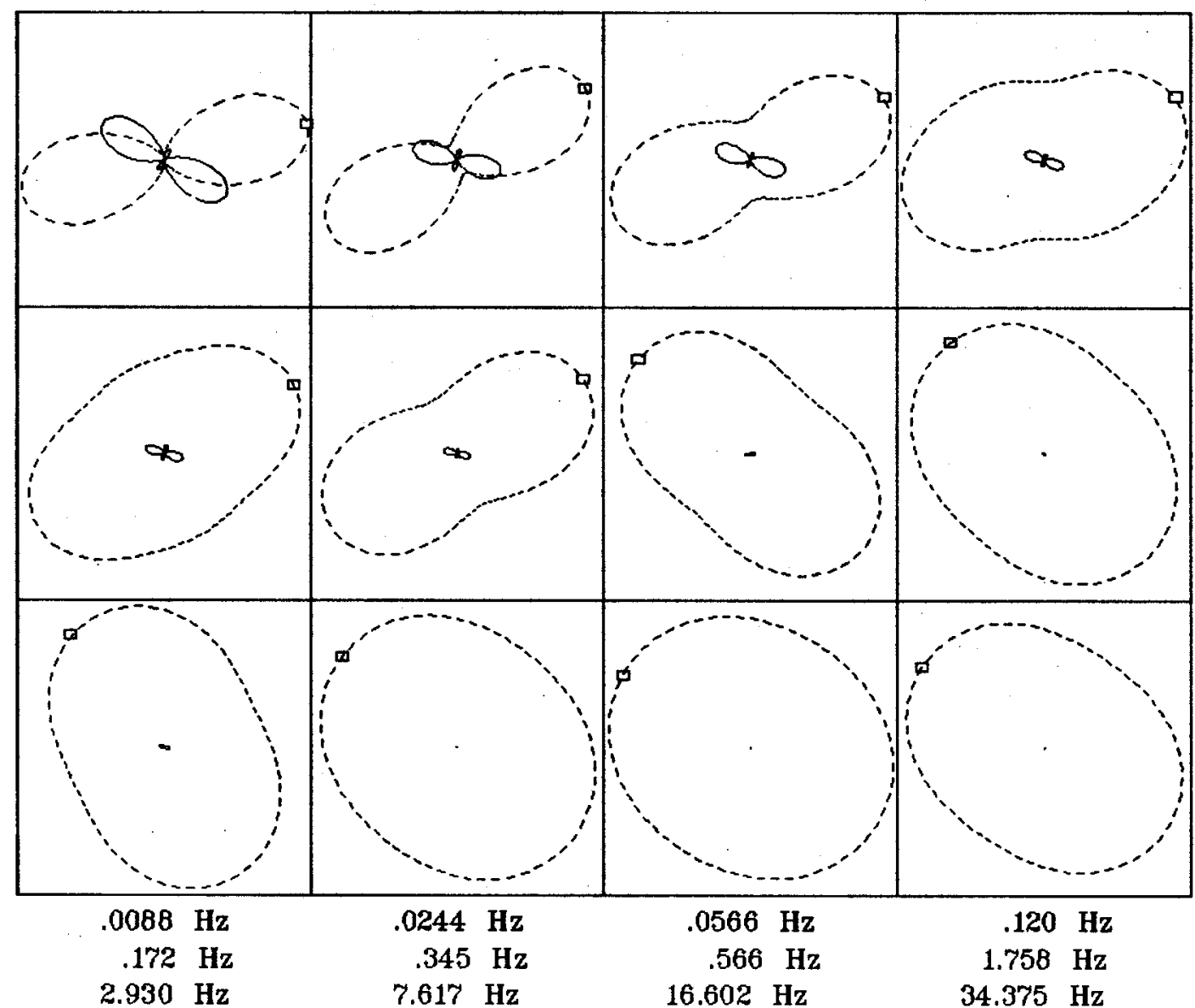

Client:

Remote: none

Acquired: 12:1 Jul 31, 2000

Survey Co:USGS

\section{Rotation:}

Filename: hr100a.avg

Channels: Ch1 Ch2 Ch3 Ch4 Ch5 Ch3 Ch4 Plotted: 15:25 Jan 24, 2001

< EMI - ElectroMagnetic Instruments > 
TIPPER MAGNITUDE

Crescent Valley, NV 100K

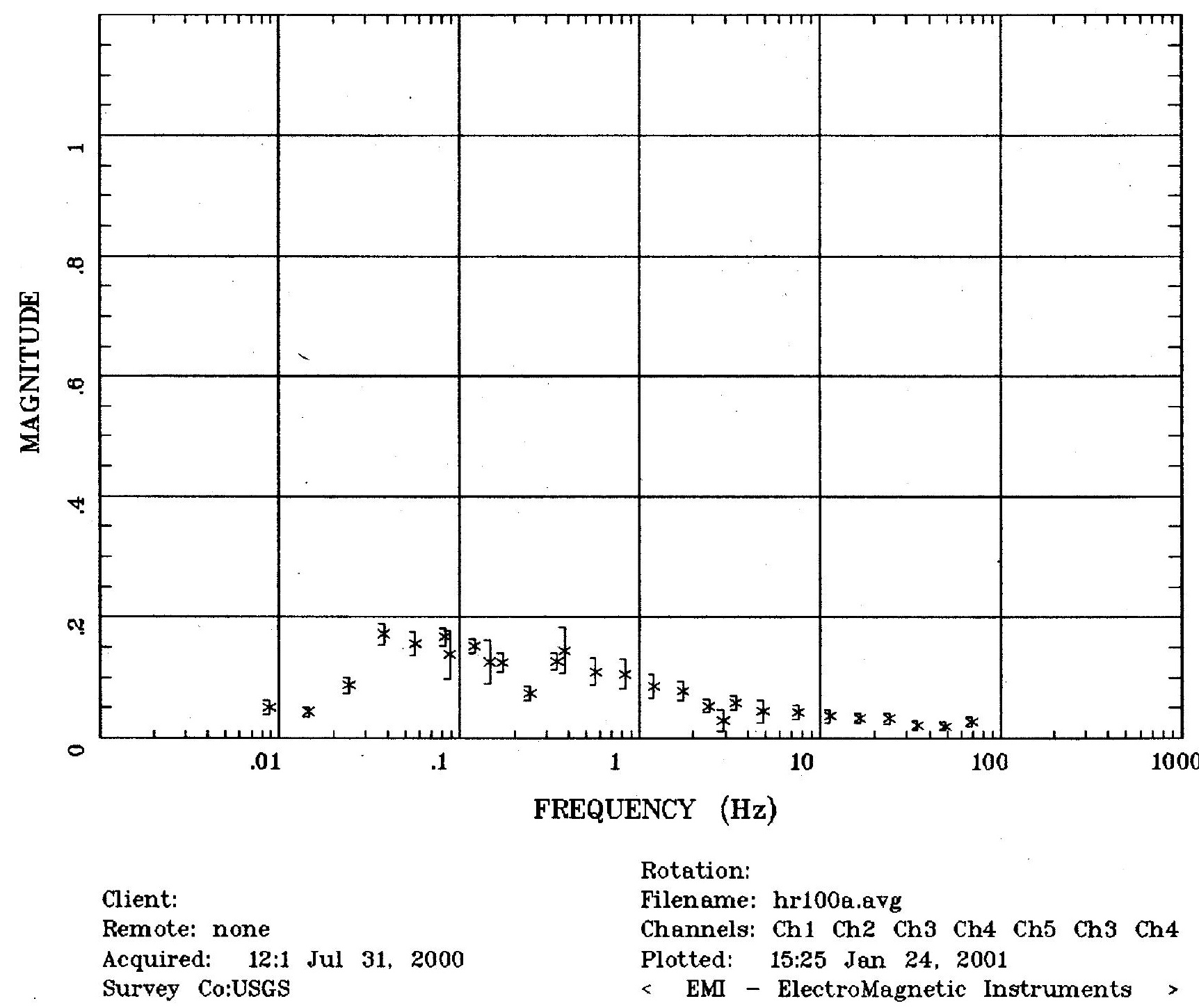


TIPPER STRIKE

Crescent Valley, NV 100K

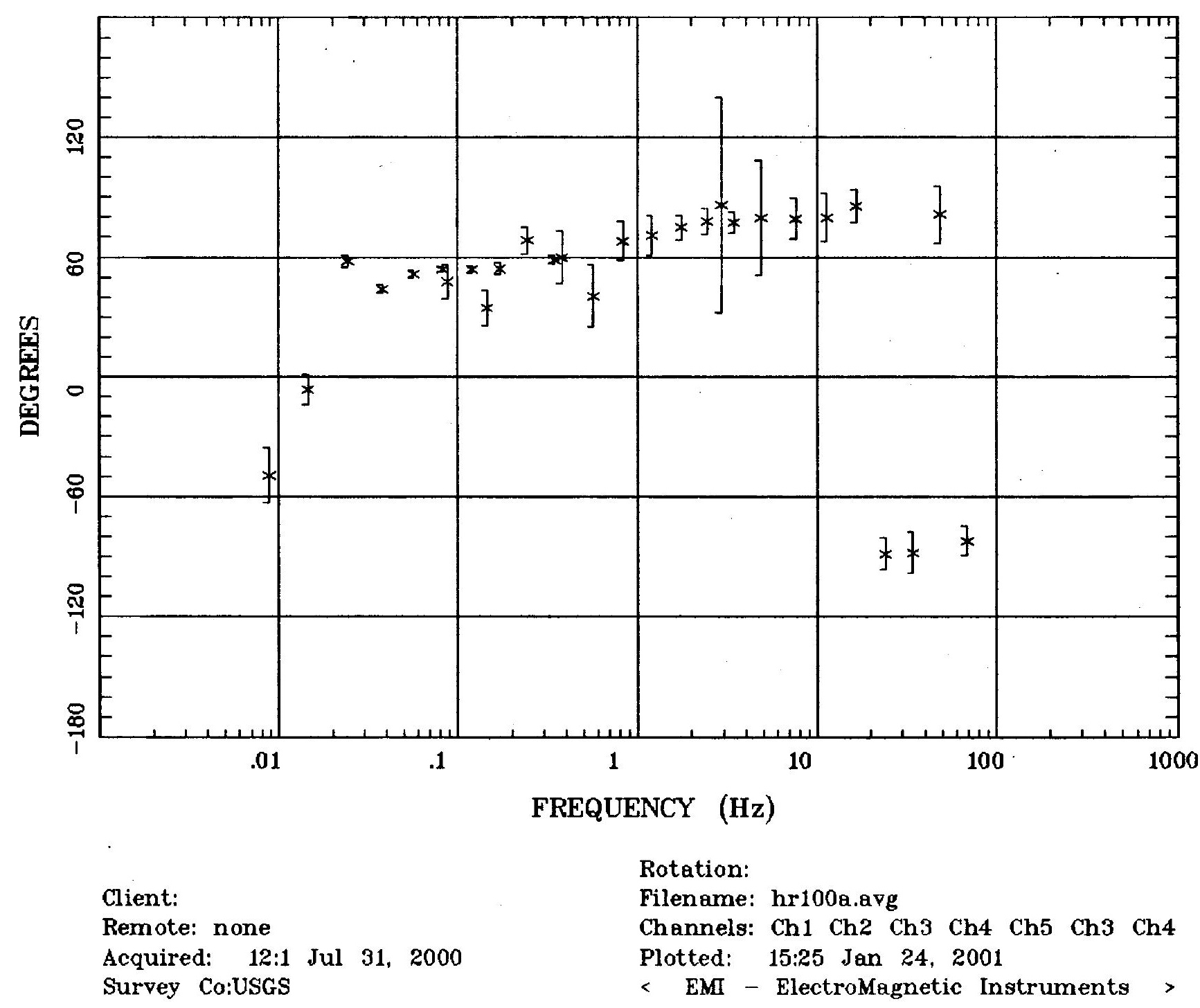


HzHx.x Coh HzHy.o

Crescent Valley, NV 100K

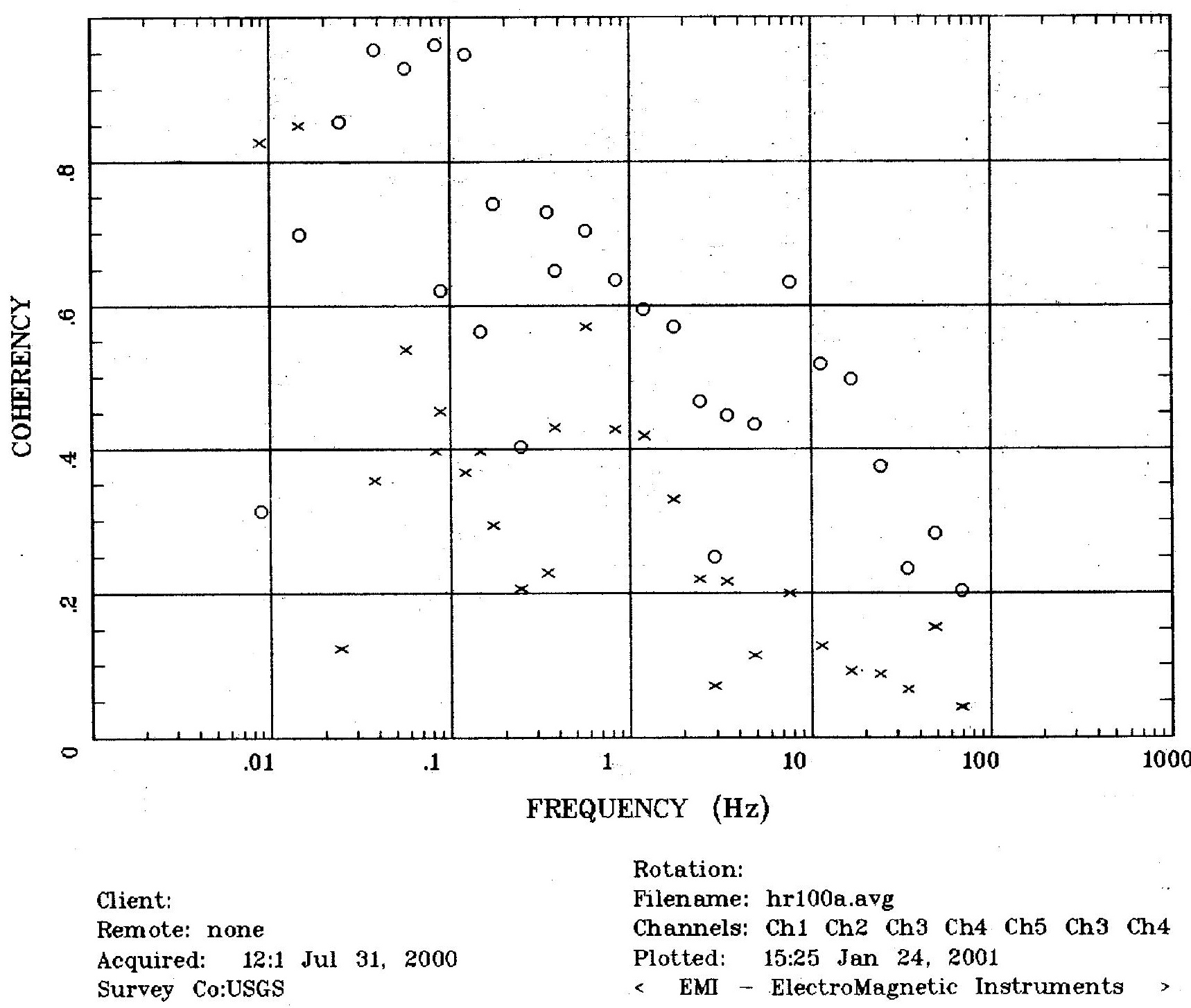




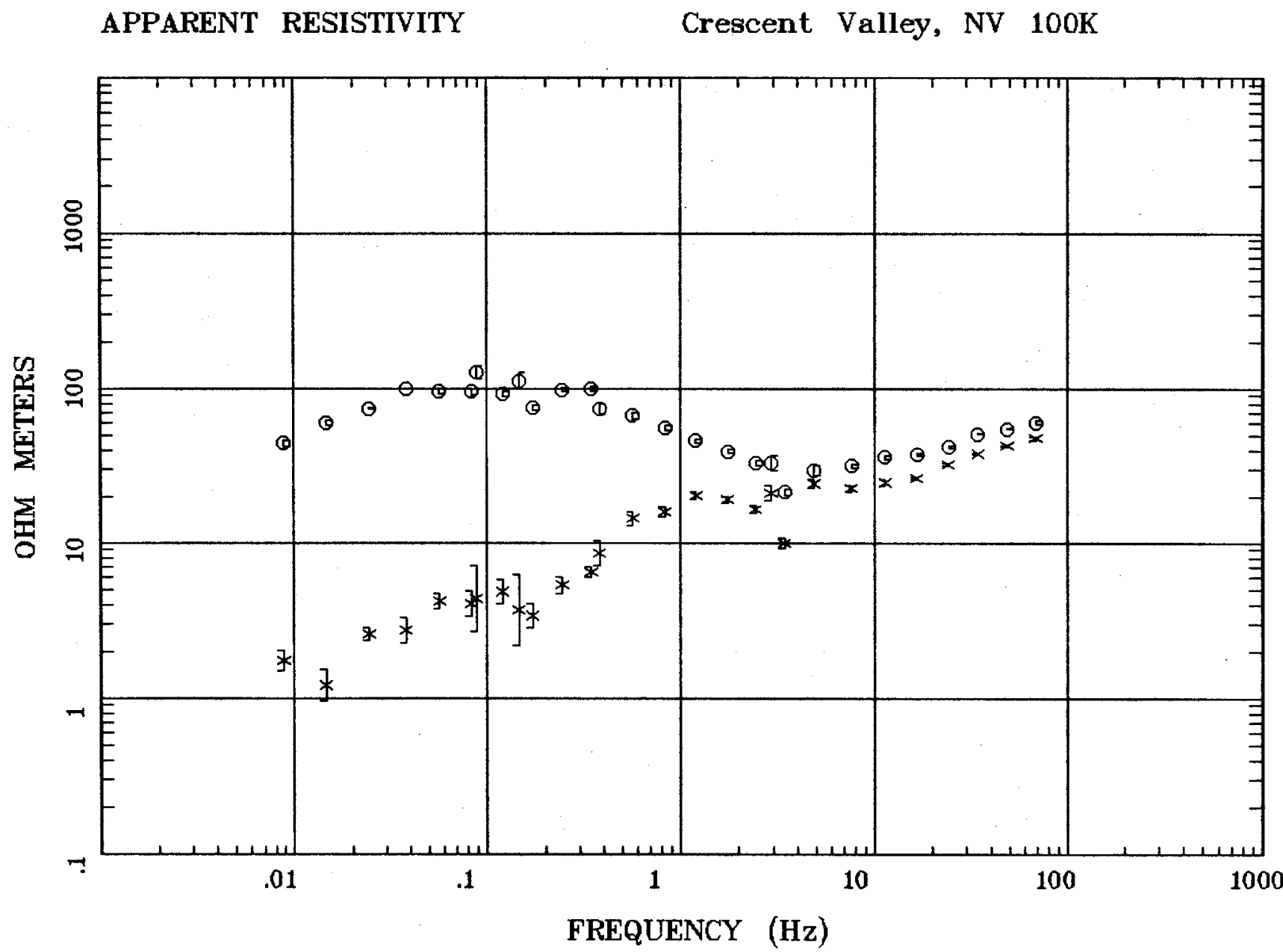

Client:

Remote: none

Acquired: 09:4 Aug 02, 2000

Survey Co:USGS
Rotation:

Filename: hr101all.avg

Channels: Ch1 Ch2 Ch3 Ch4 Ch5 Ch3 Ch4

Platted: 15:26 Jan 24, 2001

< EMI - ElectroMagnetic Instruments 
IMPEDANCE PHASE

Crescent Valley, NV 100K

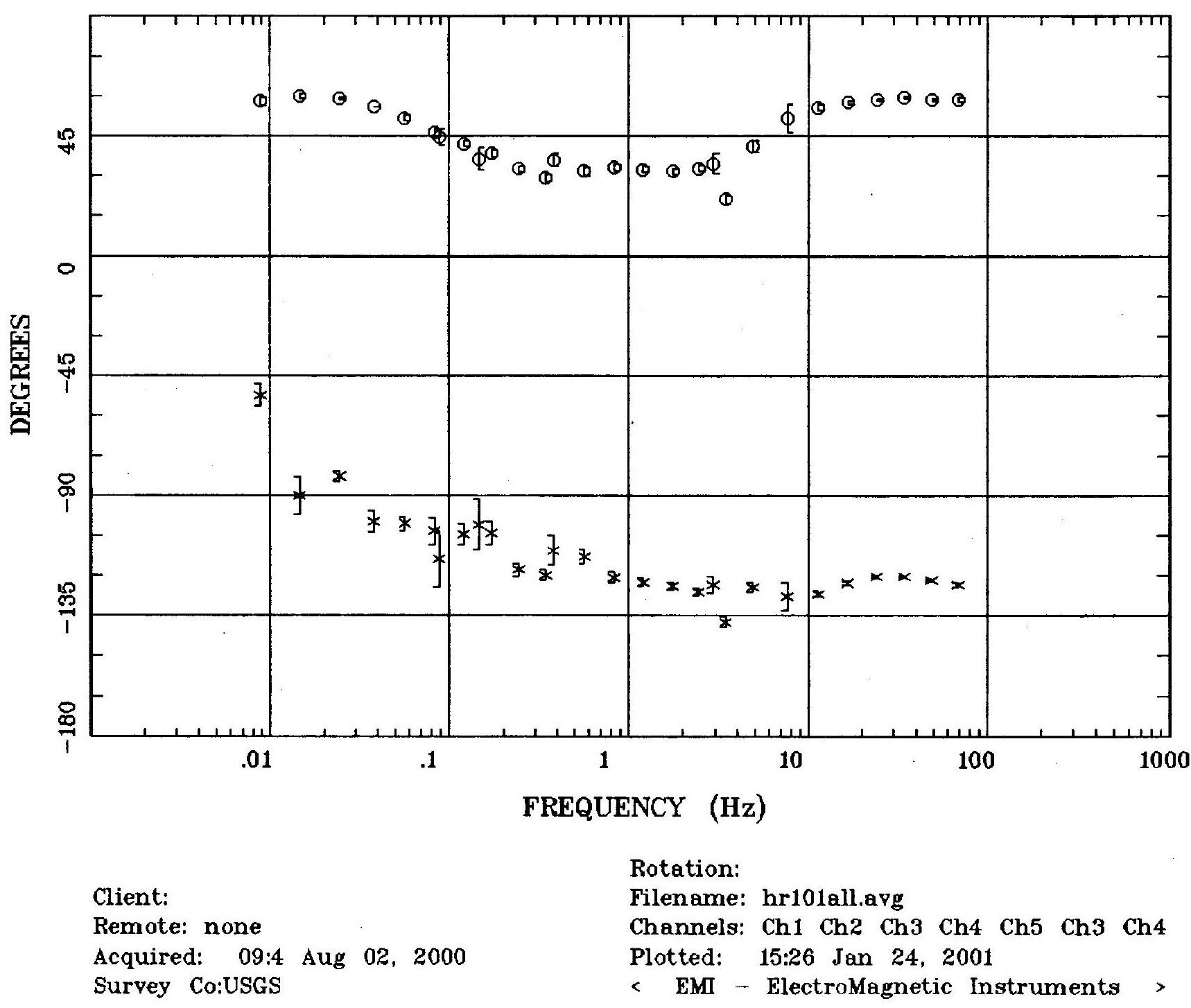




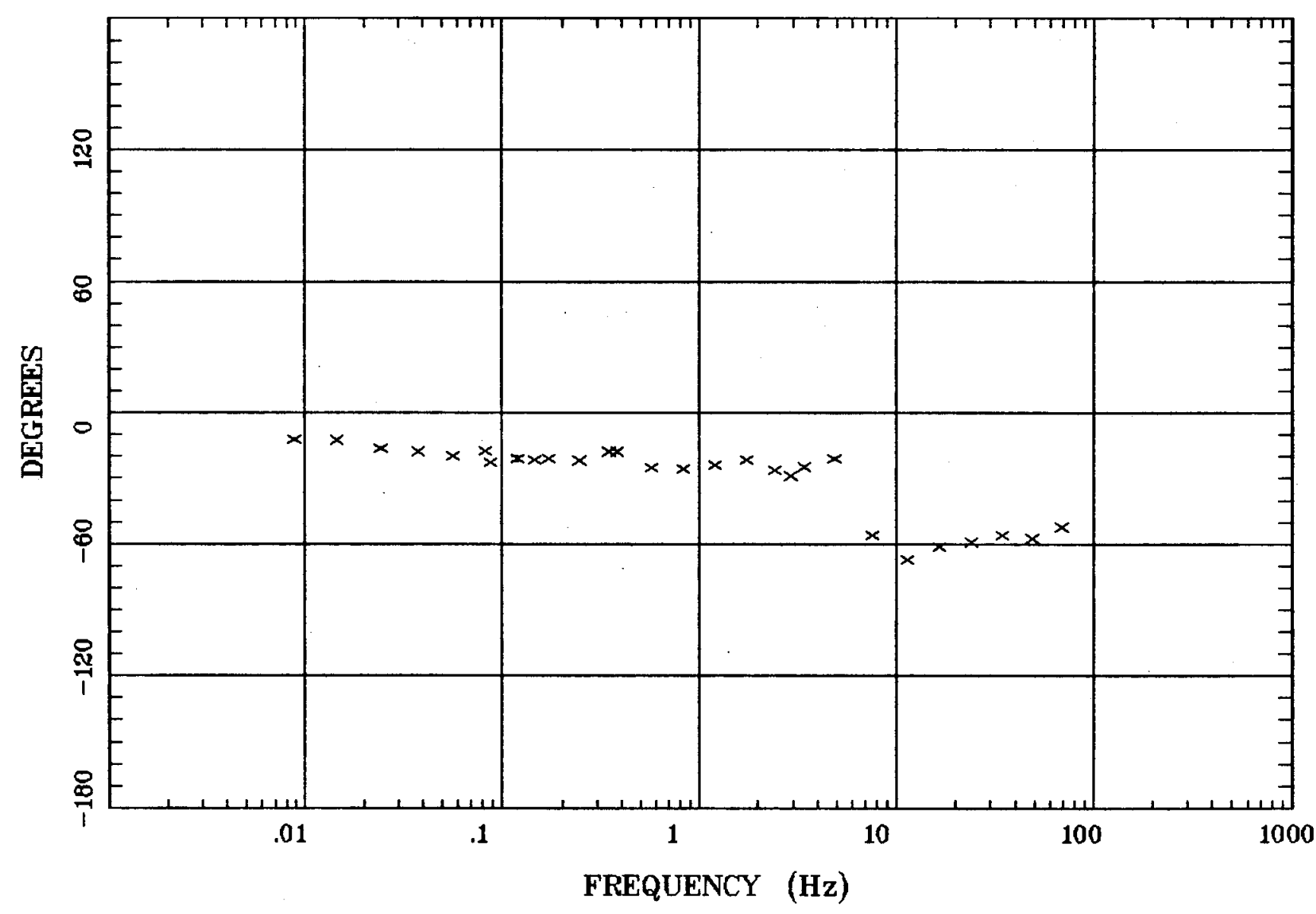

Client:

Remote: none

Acquired: 09:4 Aug 02, 2000

Survey Co:USGS
Rotation:

Filename: hr101all.avg

Channels: Ch1 Ch2 Ch3 Ch4 Ch5 Ch3 Ch4

Plotted: 15:26 Jan 24, 2001

< EMI - ElectroMagnetic Instruments 


\section{Station 101}

IMPEDANCE SKEW

Crescent Valley, NV 100K

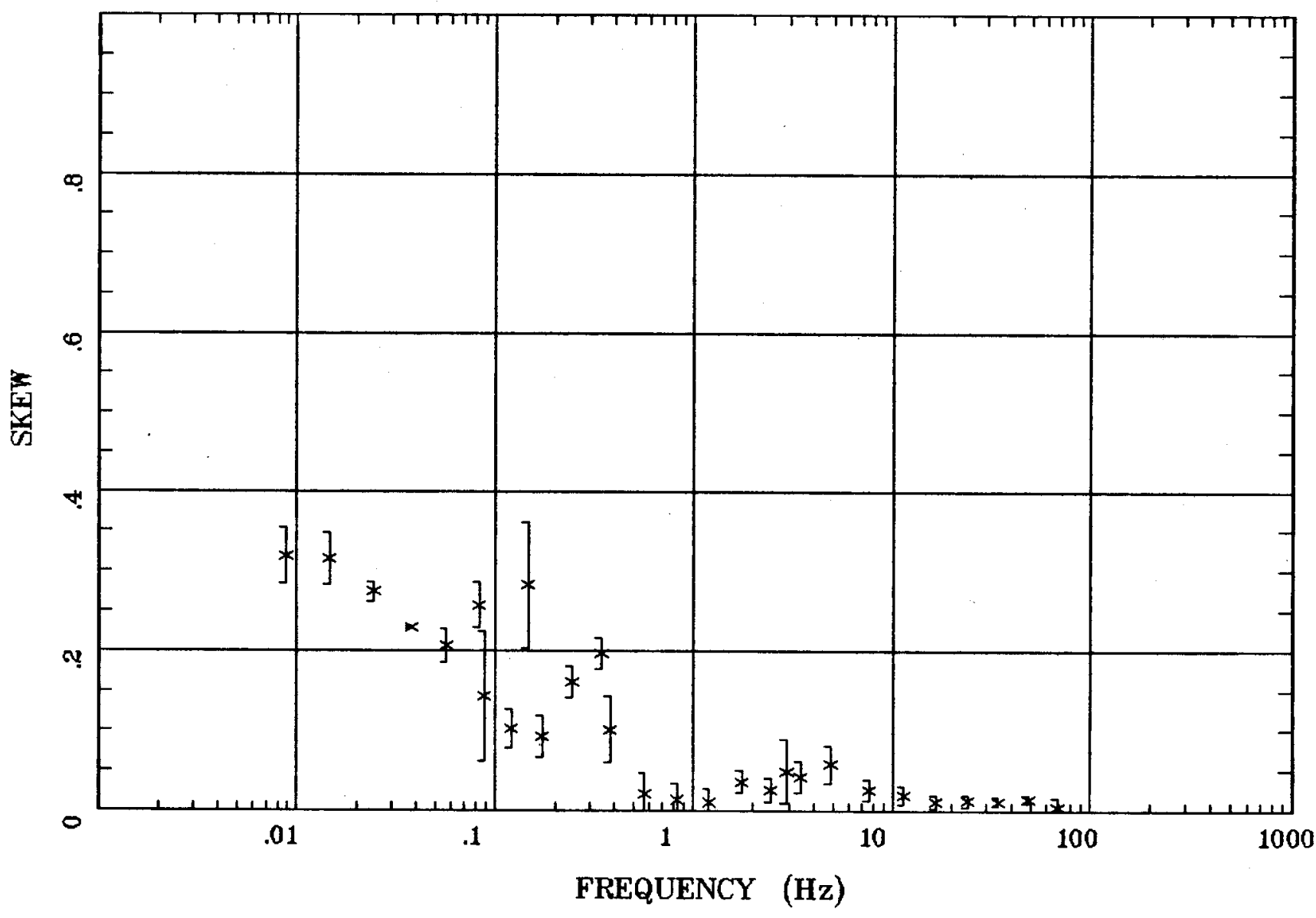

Client:

Remote: none

Acquired: 09:4 Aug 02, 2000

Survey Ca:USGS
Rotation:

Filename: hr101all.avg

Channels: Ch1 Ch2 Ch3 Ch4 Ch5 Ch3 Ch4

Plotted: 15:26 Jan 24, 2001

$<$ EMI - ElectroMagnetic Instruments 
E MULT Coh.

Crescent Valley, NV 100K

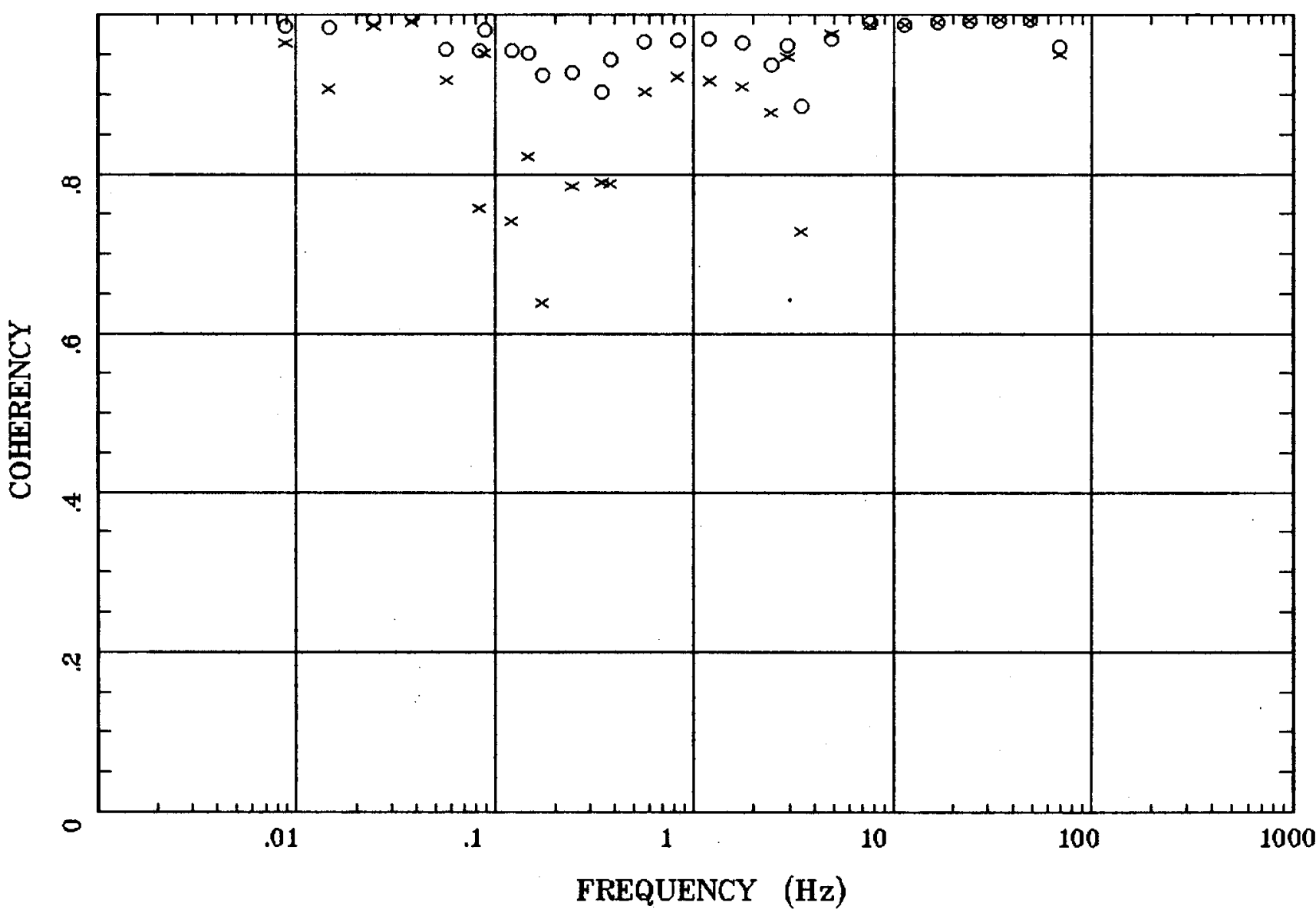

Client:

Remote: none

Acquired: 09:4 Aug 02, 2000

Survey Co:USGS
Rotation:

Filename: hr101all.avg

Channels: Ch1 Ch2 Ch3 Ch4 Ch5 Ch3 Ch4

Plotted: 15:26 Jan 24, 2001

< EMI - ElectroMagnetic Instruments 


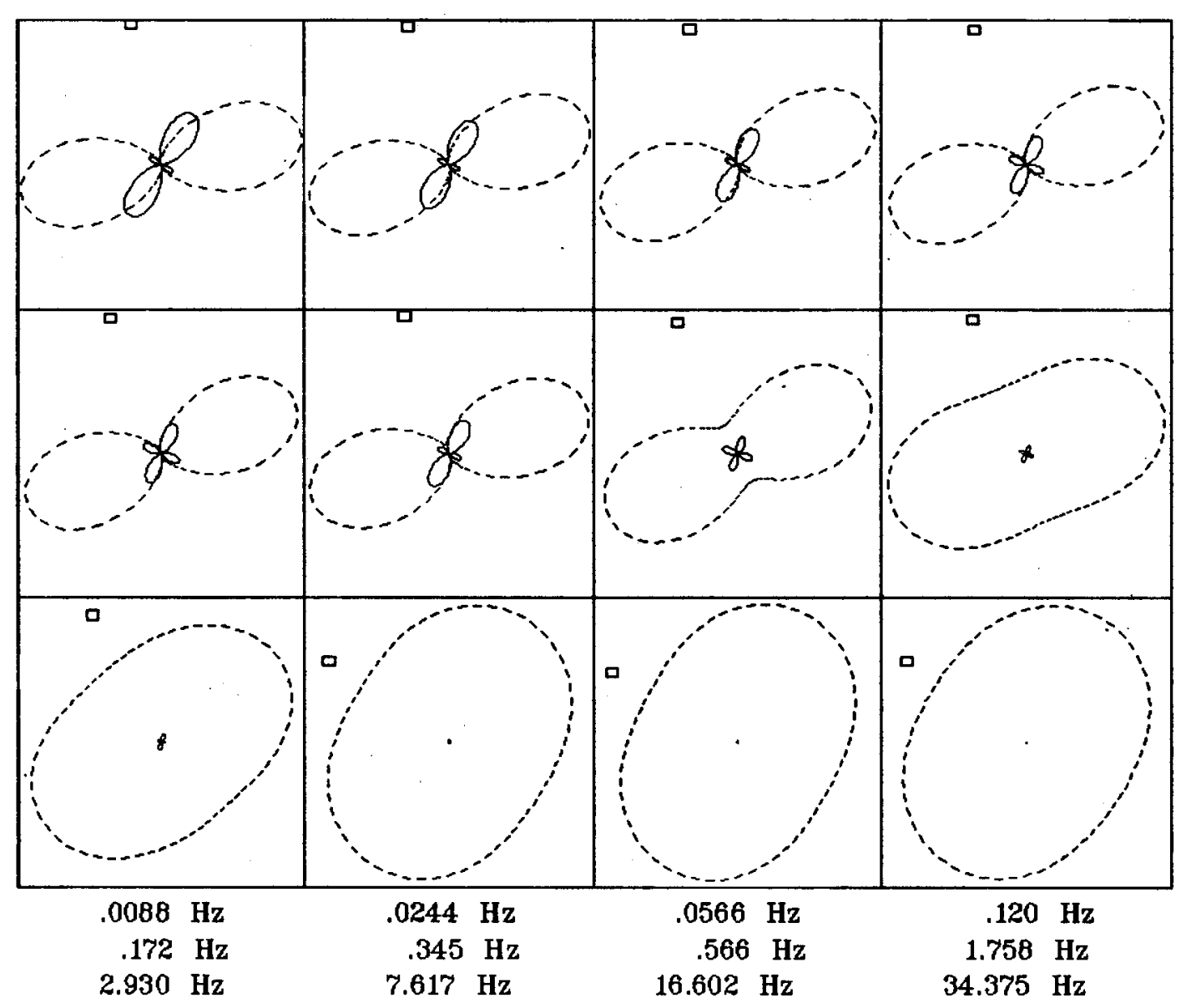

Client:

Remote: none

Acquired: 09:4 Aug 02, 2000 Survey Co:USGS
Rotation:

Filename: hr101all.avg

Channels: Ch1 Ch2 Ch3 Ch4 Ch5 Ch3 Ch4

Plotted: 15:26 Jan 24, 2001

< EMI - ElectroMagnetic Instruments > 
TIPPER MAGNITUDE

Crescent Valley, NV 100K

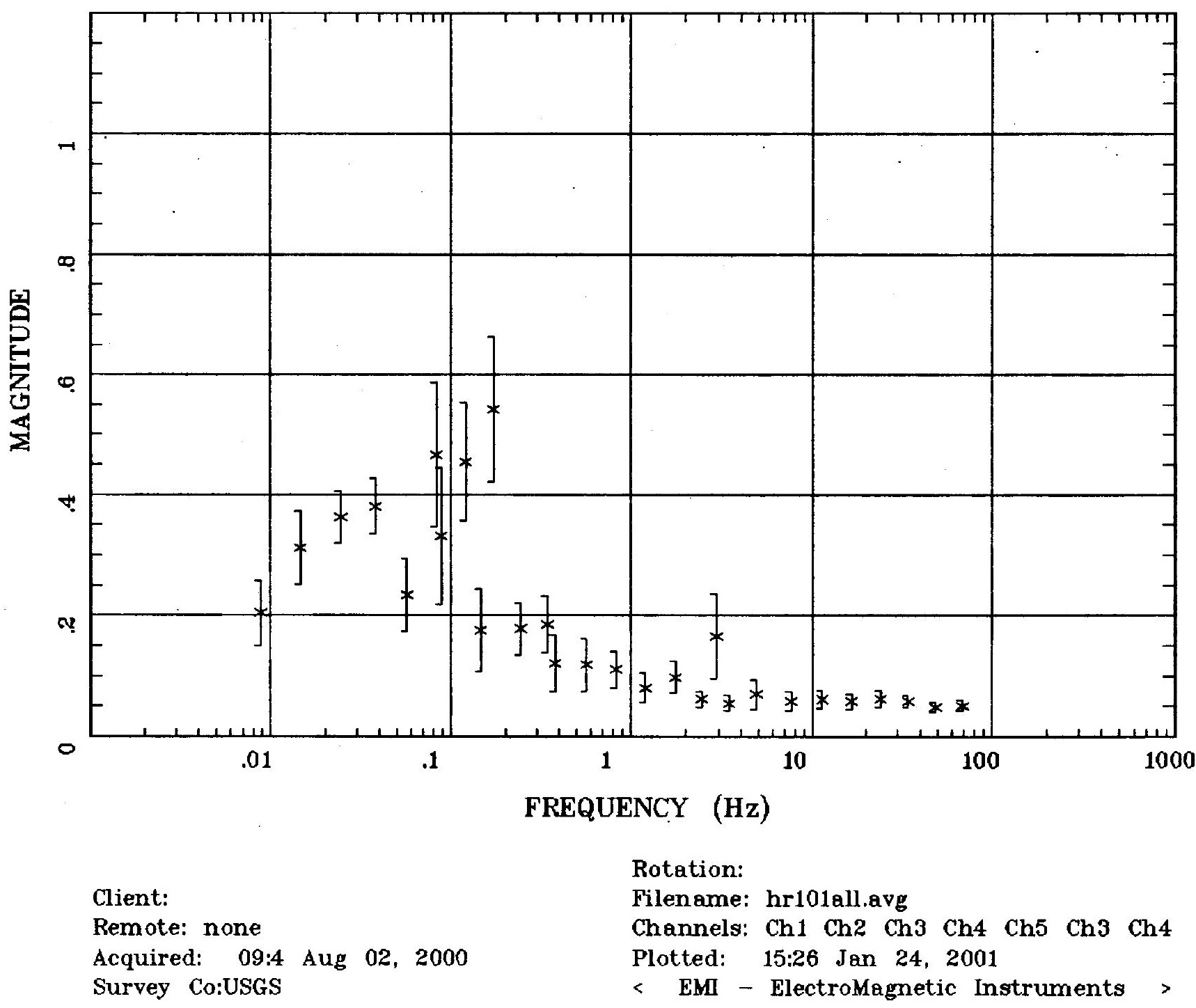


TIPPER STRIKE

Crescent Valley, NV 100K

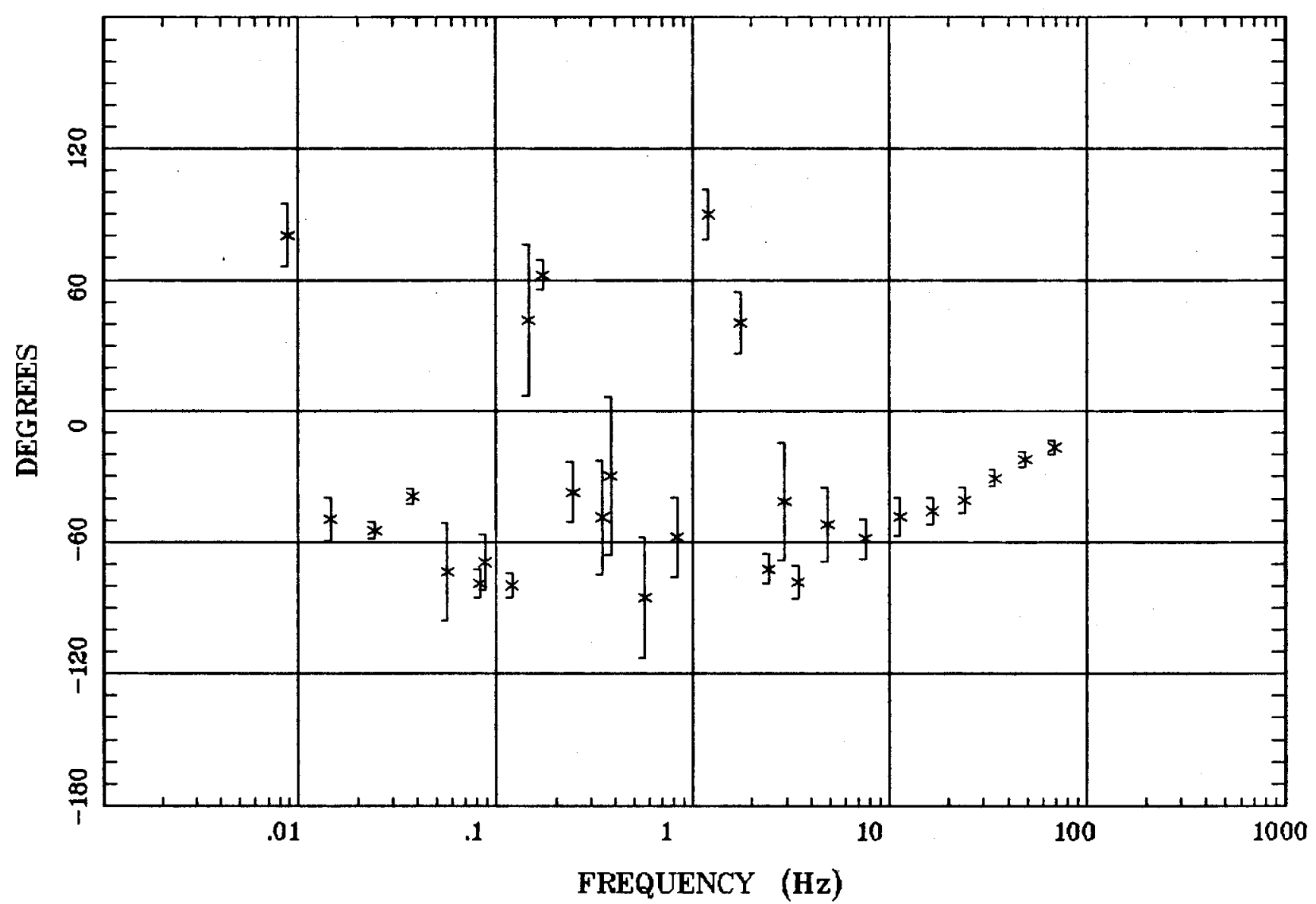

Client:

Remate: none

Acquired: 09:4 Aug 02, 2000

Survey Co:USGS
Rotation:

Filename: hr101all.avg

Channels: Ch1 Ch2 Ch3 Ch4 Ch5 Ch3 Ch4

Plotted: 15:26 Jan 24, 2001

$<$ EMI - ElectroMagnetic Instruments > 
HzHx.x Coh HzHy.o

Crescent Valley, NV 100K

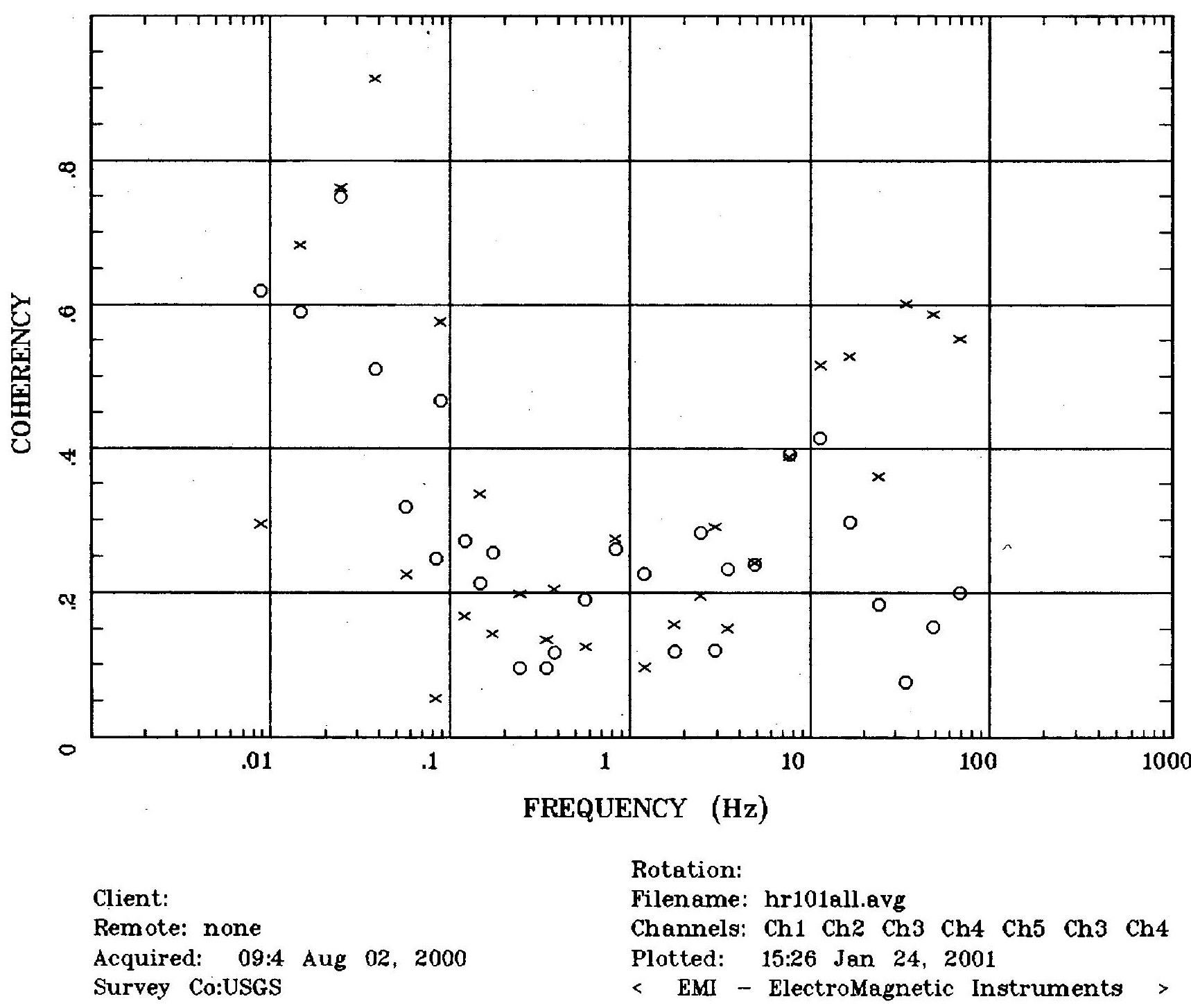




\section{APPARENT RESISTIVITY}

Crescent Valley, NV 100K

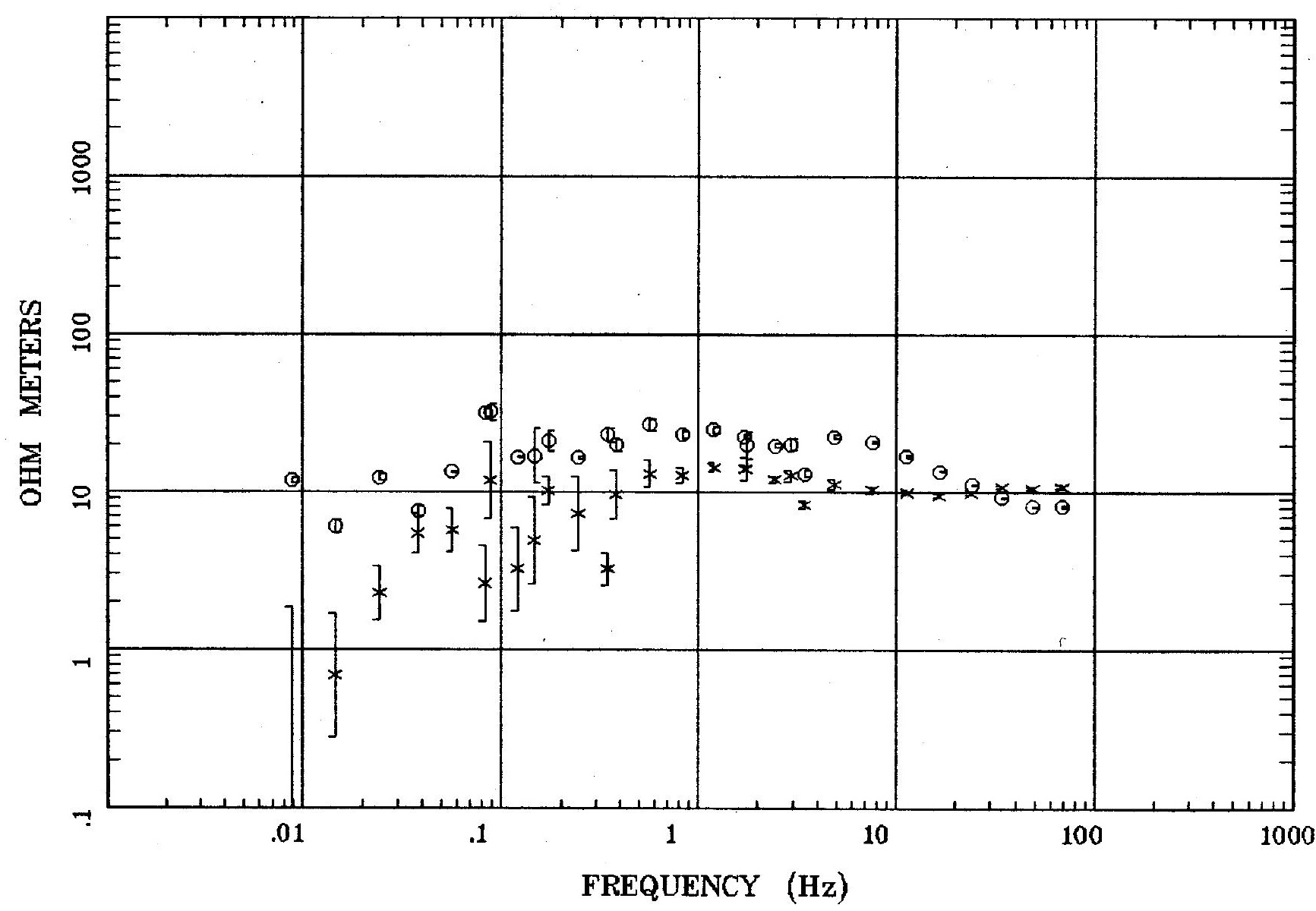

Client:

Remote: none

Acquired: 13:0 Aug 02, 2000 Survey Co:USGS
Rotation:

Filename: hr102all.avg

Channels: Ch1 Ch2 Ch3 Ch4 Ch5 Ch3 Ch4 Plotted: 15:27 Jan 24, 2001

< EMI - ElectroMagnetic Instruments 


\section{Station 102}

IMPEDANCE PHASE

Crescent Valley, NV 100K

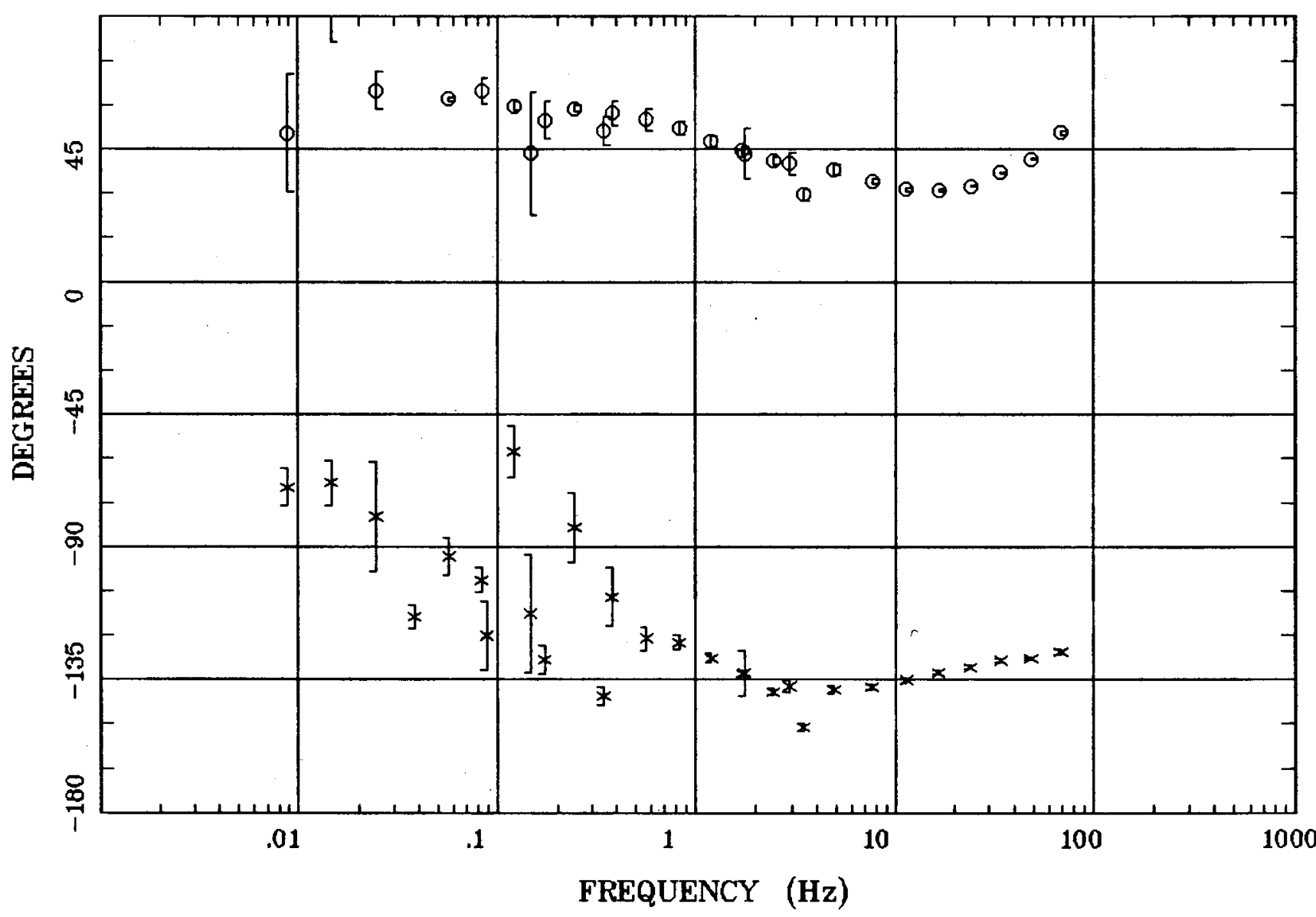

Client:

Remote: none

Acquired: 13:0 Aug 02, 2000 Survey Co:USGS
Rotation:

Filename: hr102all.avg

Channels: Ch1 Ch2 Ch3 Ch4 Ch5 Ch3 Ch4

Plotted: 15:27 Jan 24, 2001

<EMI - ElectroMagnetic Instruments > 


\section{Station 102}

ROTATION ANGLE

Crescent Valley, NV 100K

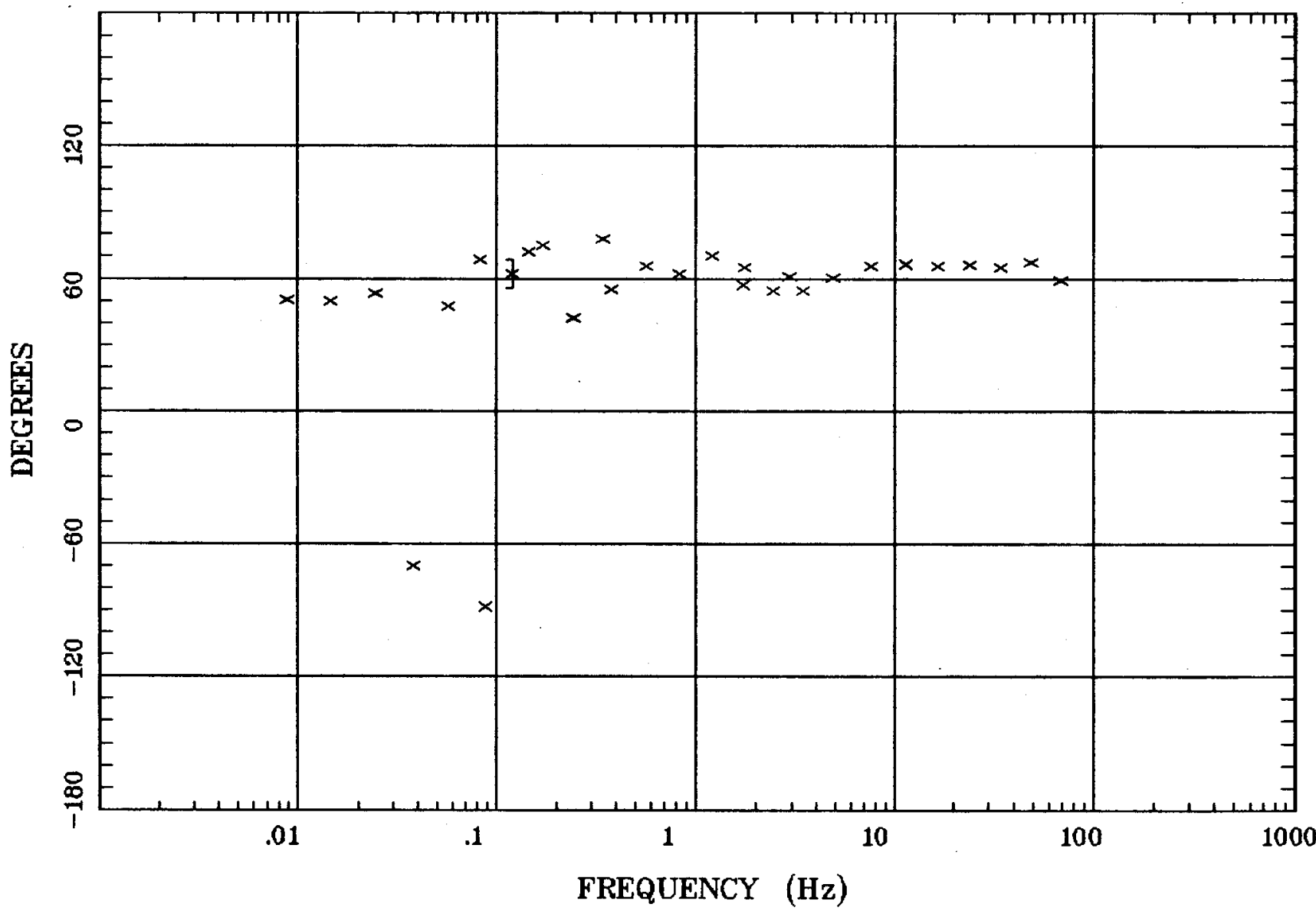

Client:

Remote: none

Acquired: 13:0 Aug 02, 2000 Survey Co:USGS
Rotation:

Filename: hr102all.avg

Channels: Ch1 Ch2 Ch3 Ch4 Ch5 Ch3 Ch4

Plotted: 15:27 Jan 24, 2001

$<$ EMI - ElectroMagnetic Instruments 
Station 102

IMPEDANCE SKEW

Crescent Valley, NV 100K

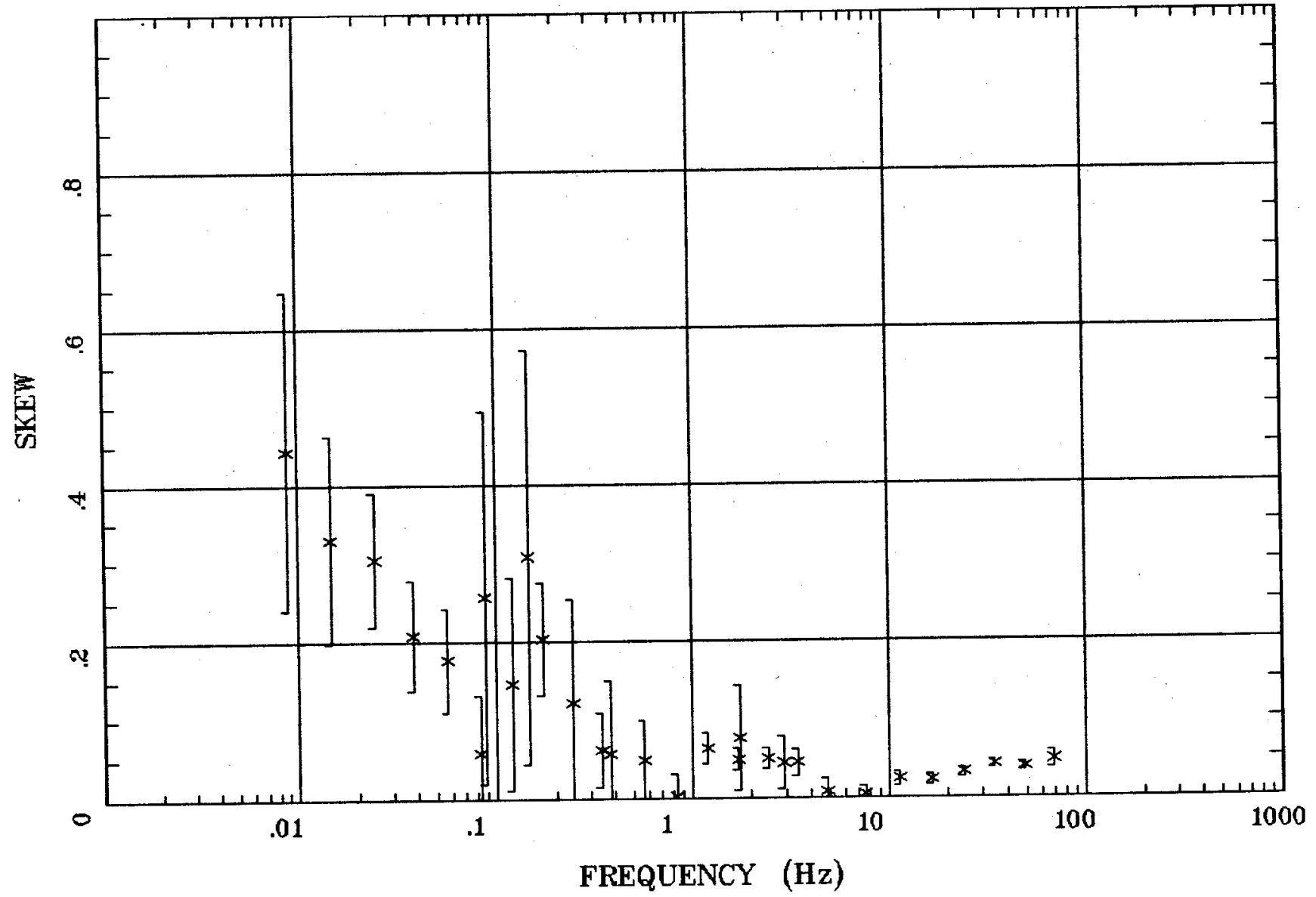

Client:

Rotation:

Remote: none

Filename: hr102all.avg

Acquired: 13:0 Aug 02, 2000

Channels: Ch1 Ch2 Ch3 Ch4 Ch5 Ch3 Ch4 Acquired: $13: 0$
Survey Co:USGS

Plotted: 15:27 Jan 24, 2001

$<$ EMI - ElectroMagnetic Instruments 


\section{Station 102}

\section{E MULT Coh.}

Crescent Valley, NV $100 \mathrm{~K}$

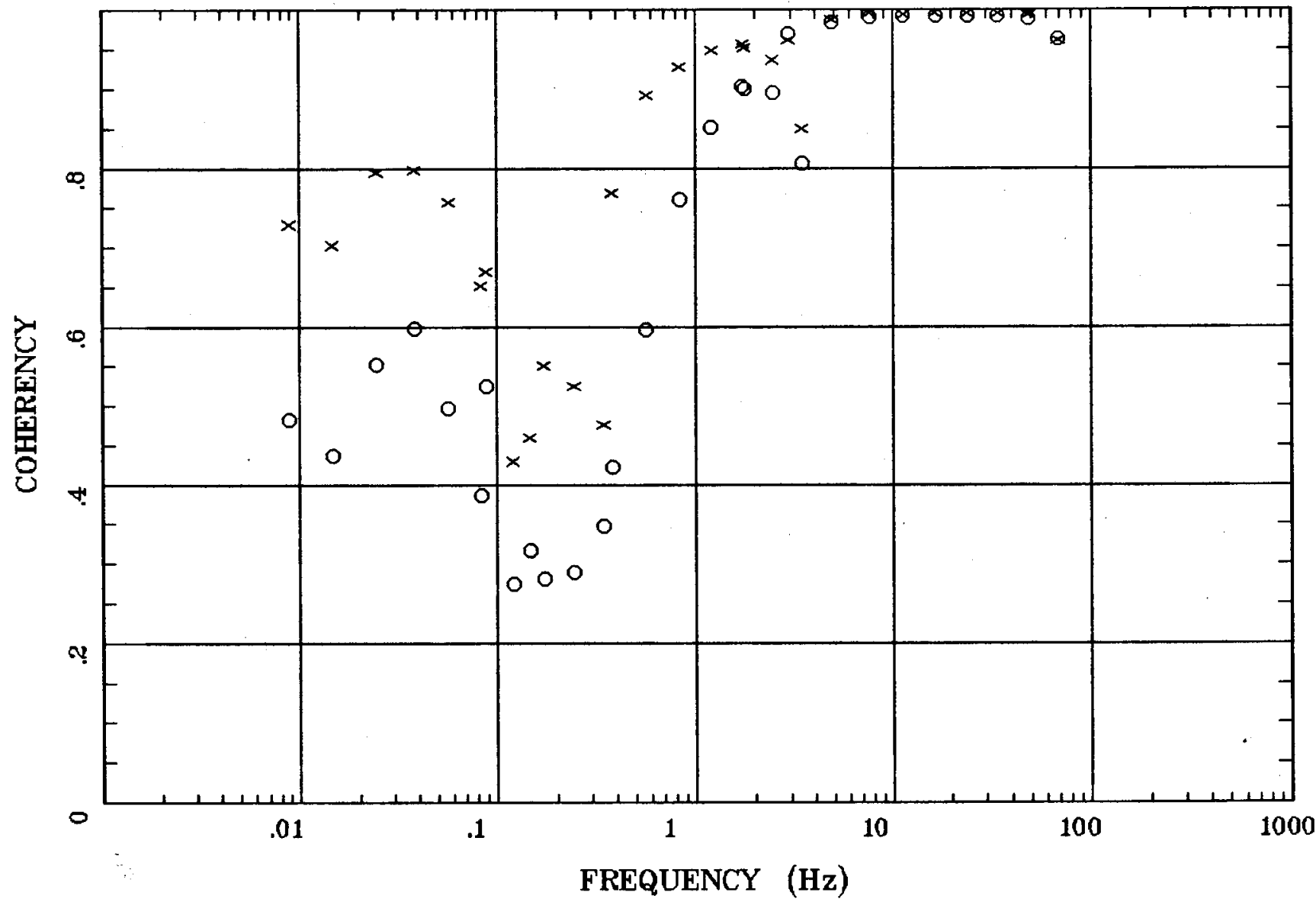

Client:

Remote: none

Acquired: 13:0 Aug 02, 2000

Survey Co:USGS

Rotation:

Filename: hr102all.avg

Channels: Ch1 Ch2 Ch3 Ch4 Ch5 Ch3 Ch4

Plotted: 15:27 Jan 24, 2001

< EMI - ElectroMagnetic Instruments > 
POLAR PLOTS

Crescent Valley, NV 100K

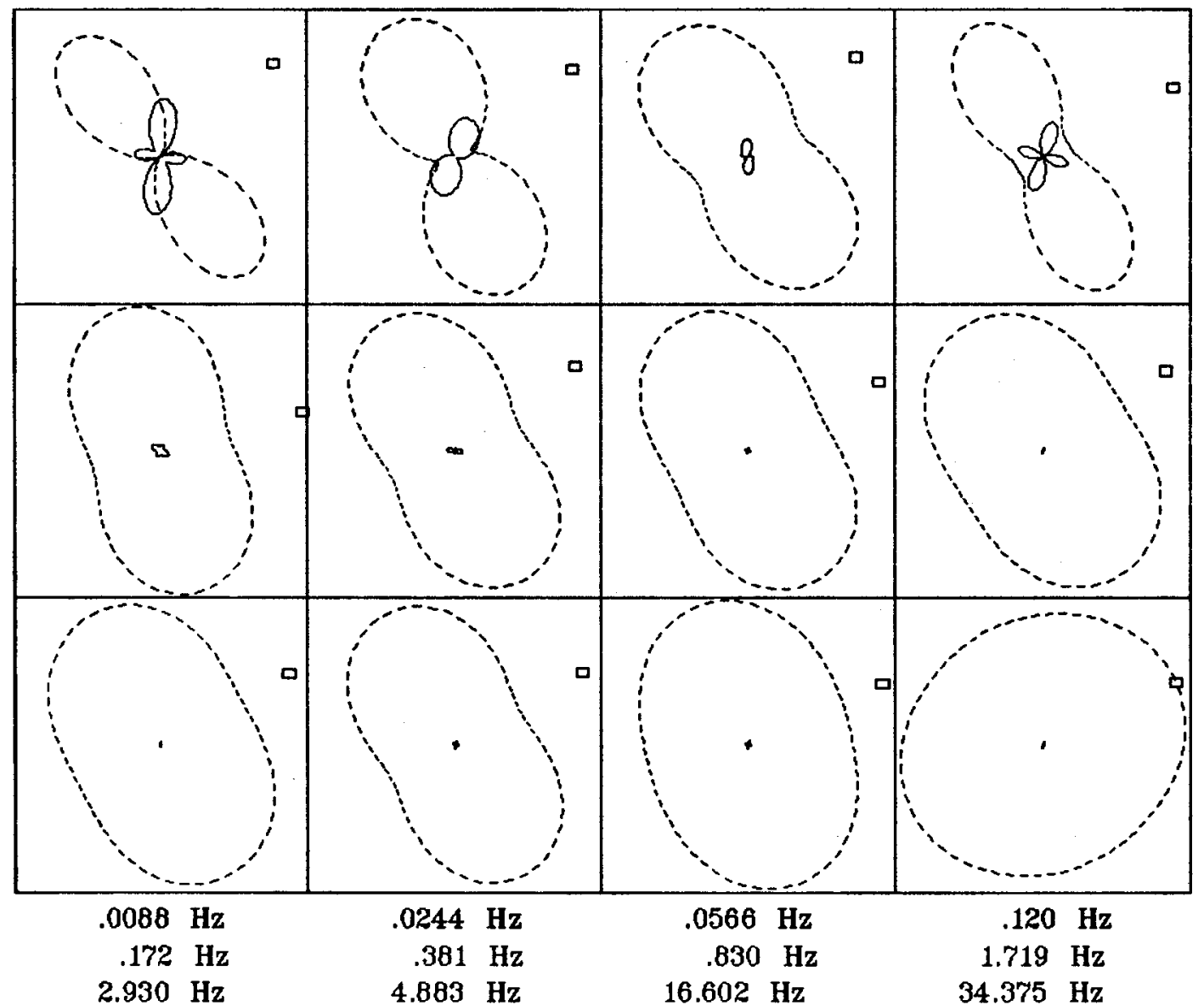

\section{Client:}

Remote: none

Acquired: 13:0 Aug 02, 2000

Survey Co:USGS
Rotation:

Filename: hr102all.avg

Channels: Ch1 Ch2 Ch3 Ch4 Ch5 Ch3 Ch4 Plotted: 15:27 Jan 24, 2001

< EMI - ElectroMagnetic Instruments > 
TIPPER MAGNITUDE

Crescent Valley, NV 100K

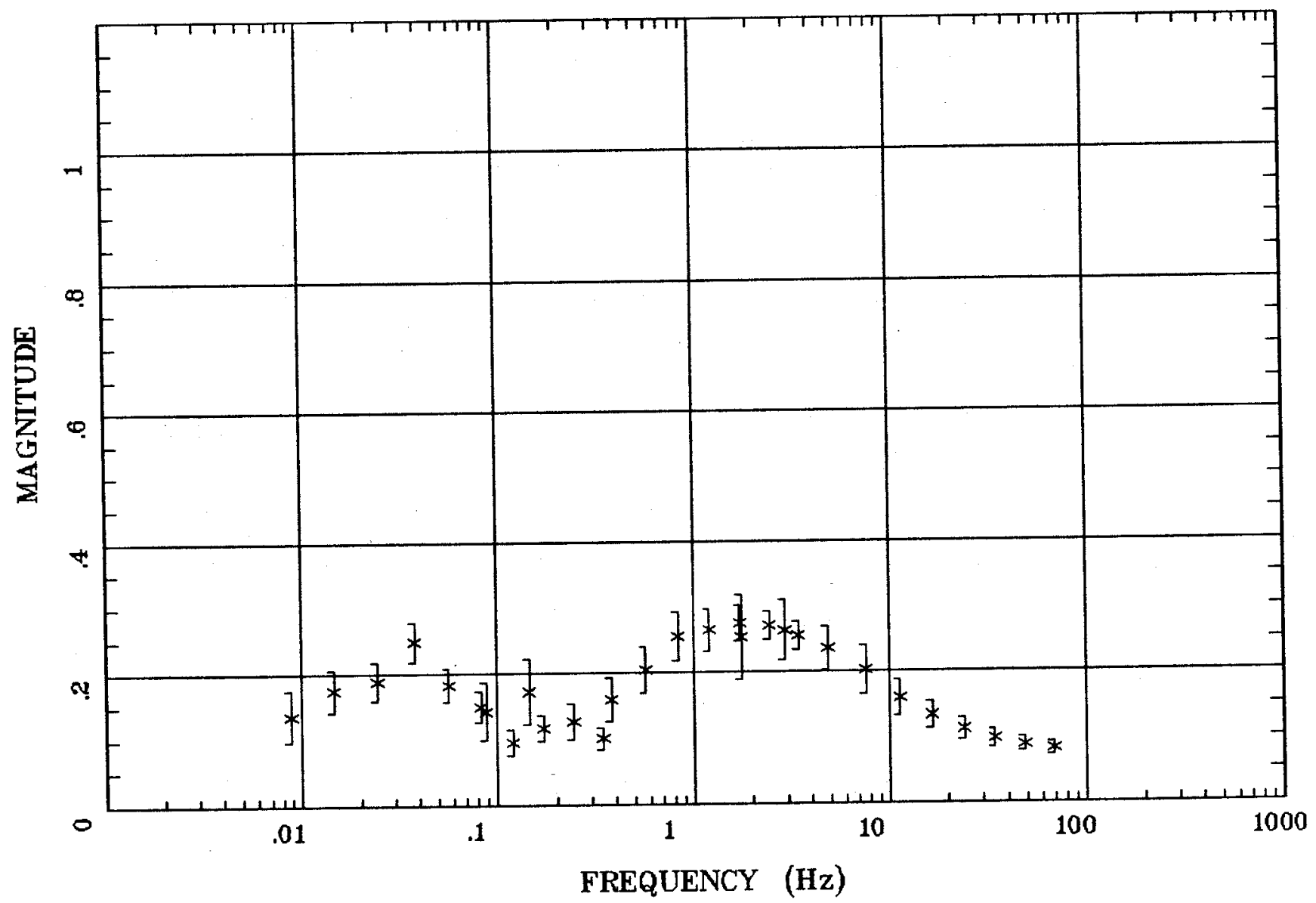

Client:

Remote: none

Acquired: 13:0 Aug 02, 2000

Survey Co:USGS

Rotation:

Filename: hr102all.avg

Channels: Ch1 Ch2 Ch3 Ch4 Ch5 Ch3 Ch4

Plotted: 15:27 Jan 24, 2001

< EMI - ElectroMagnetic Instruments 


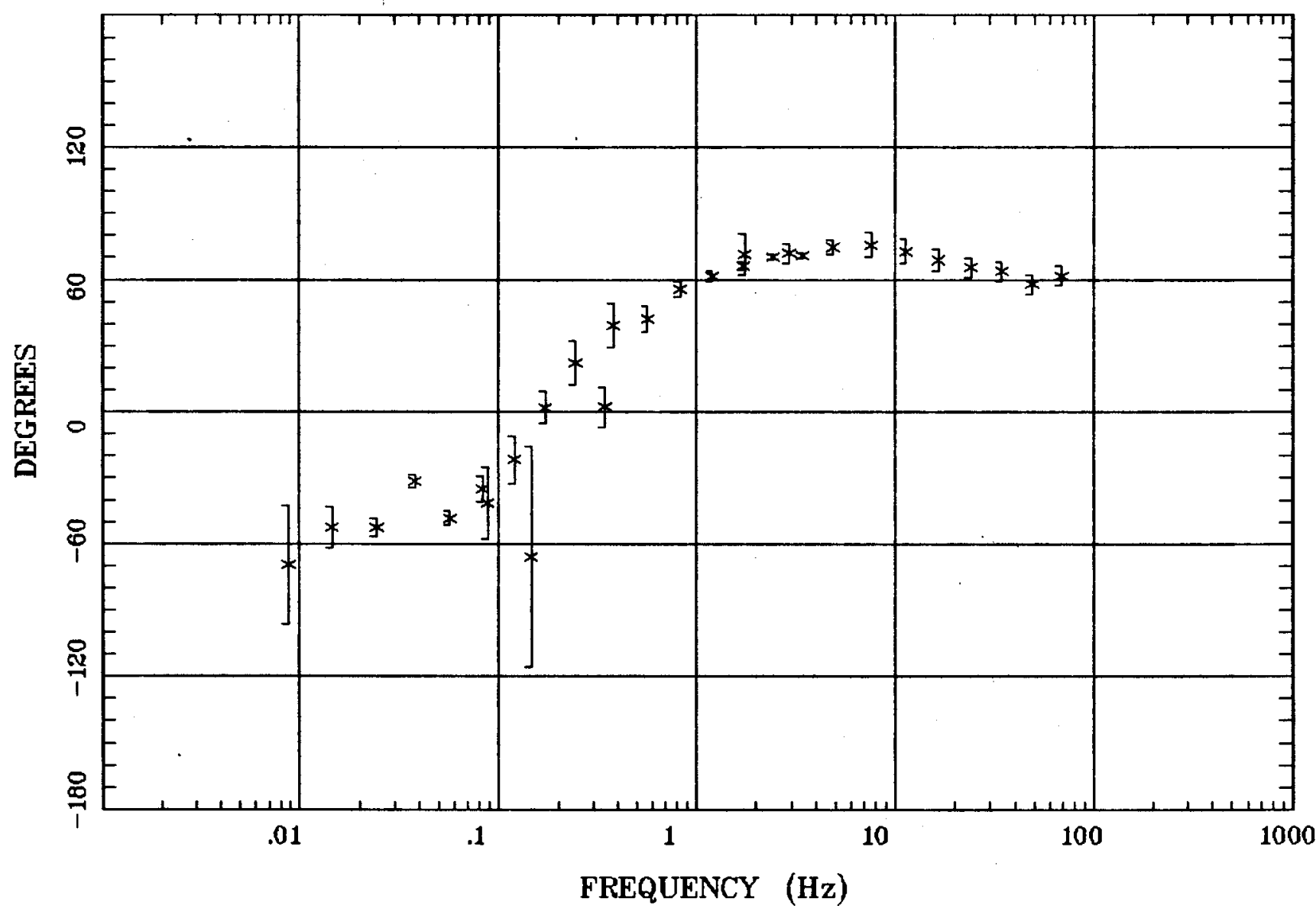

Client:

Remote: none

Acquired: 13:0 Aug 02, 2000 Survey Co:USGS
Rotation:

Filename: hr102all.avg

Channels: Ch1 Ch2 Ch3 Ch4 Ch5 Ch3 Ch4

Plotted: 15:27 Jan 24, 2001

< EMI - ElectroMagnetic Instruments > 
HzHx.x Coh HzHy.o

Crescent Valley, NV 100K

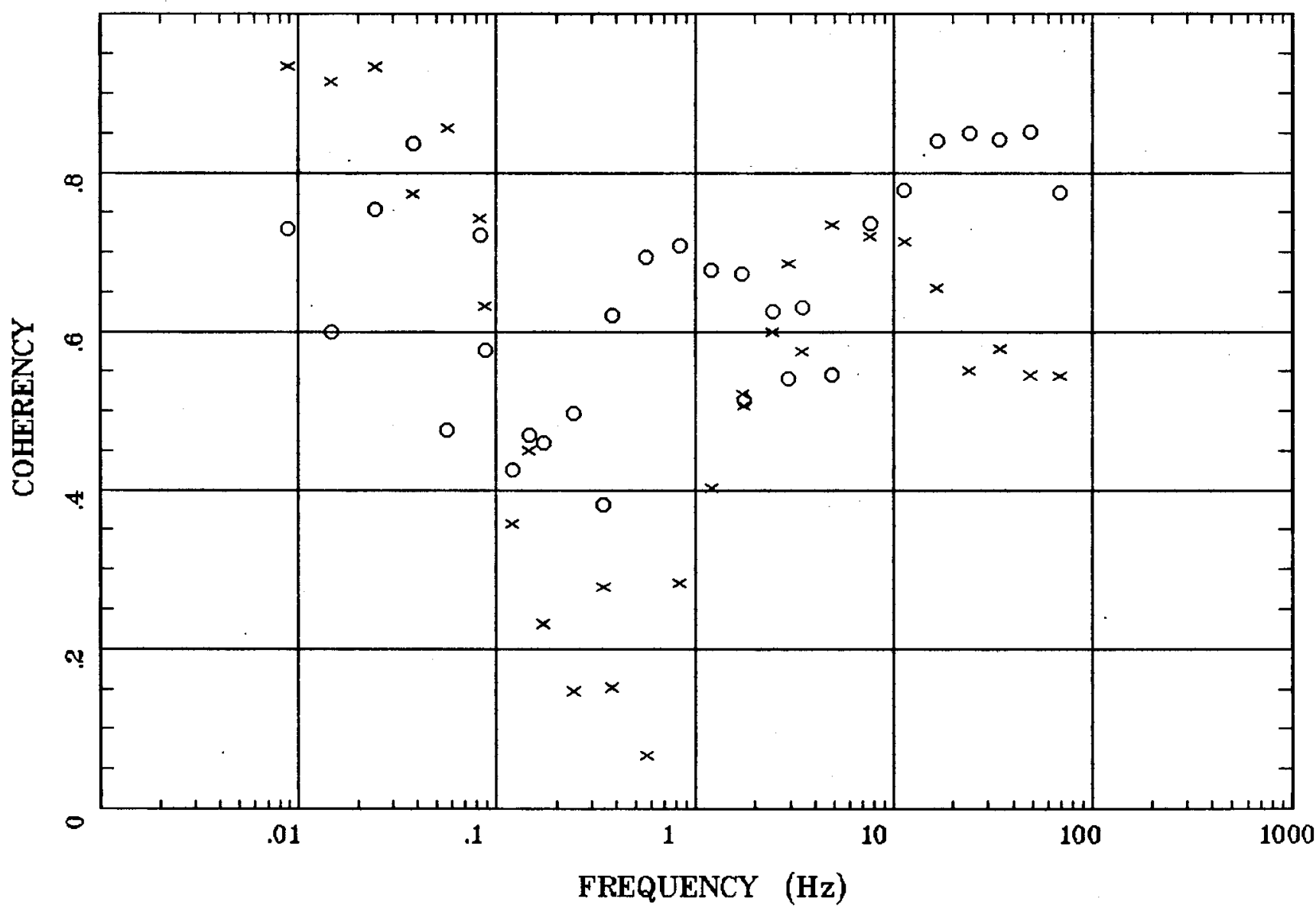

Client:

Remote: none

Acquired: 13:0 Aug 02, 2000

Survey Co:USGS
Rotation:

Filename: hr102all.avg

Channels: Ch1 Ch2 Ch3 Ch4 Ch5 Ch3 Ch4

Plotted: 15:27 Jan 24, 2001

< EMI - ElectroMagnetic Instruments > 
APPARENT RESISTIVITY Crescent Valley, NV 100K

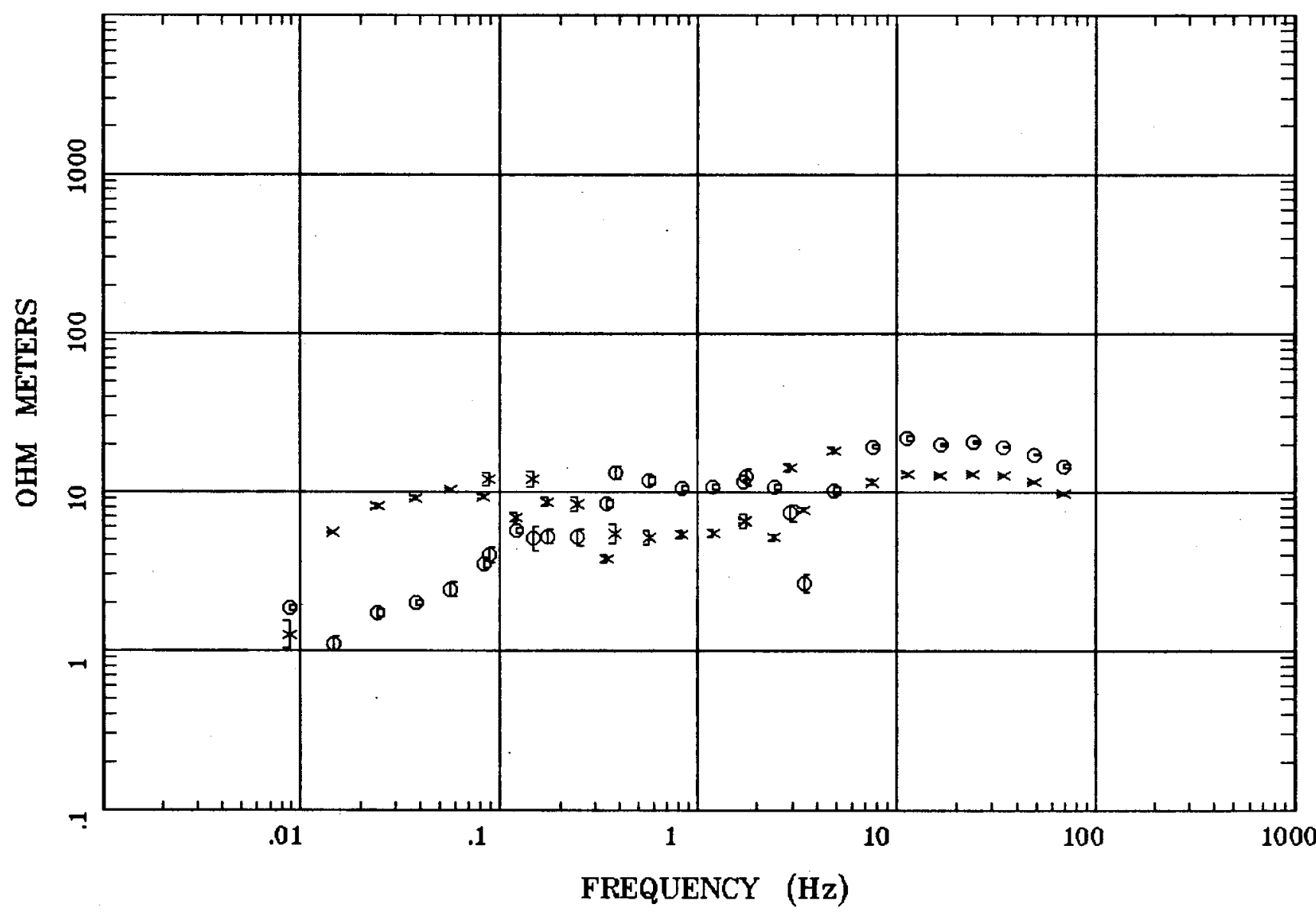

Client:

Remote: none

Acquired: 10:2 Aug 03, 2000

Survey Co:USGS
Rotation:

Filename: hr103all.avg

Channels: Ch1 Ch2 Ch3 Ch4 Ch5 Ch3 Ch4

Plotted: 15:28 Jan 24, 2001

$<$ EMI - ElectroMagnetic Instruments 


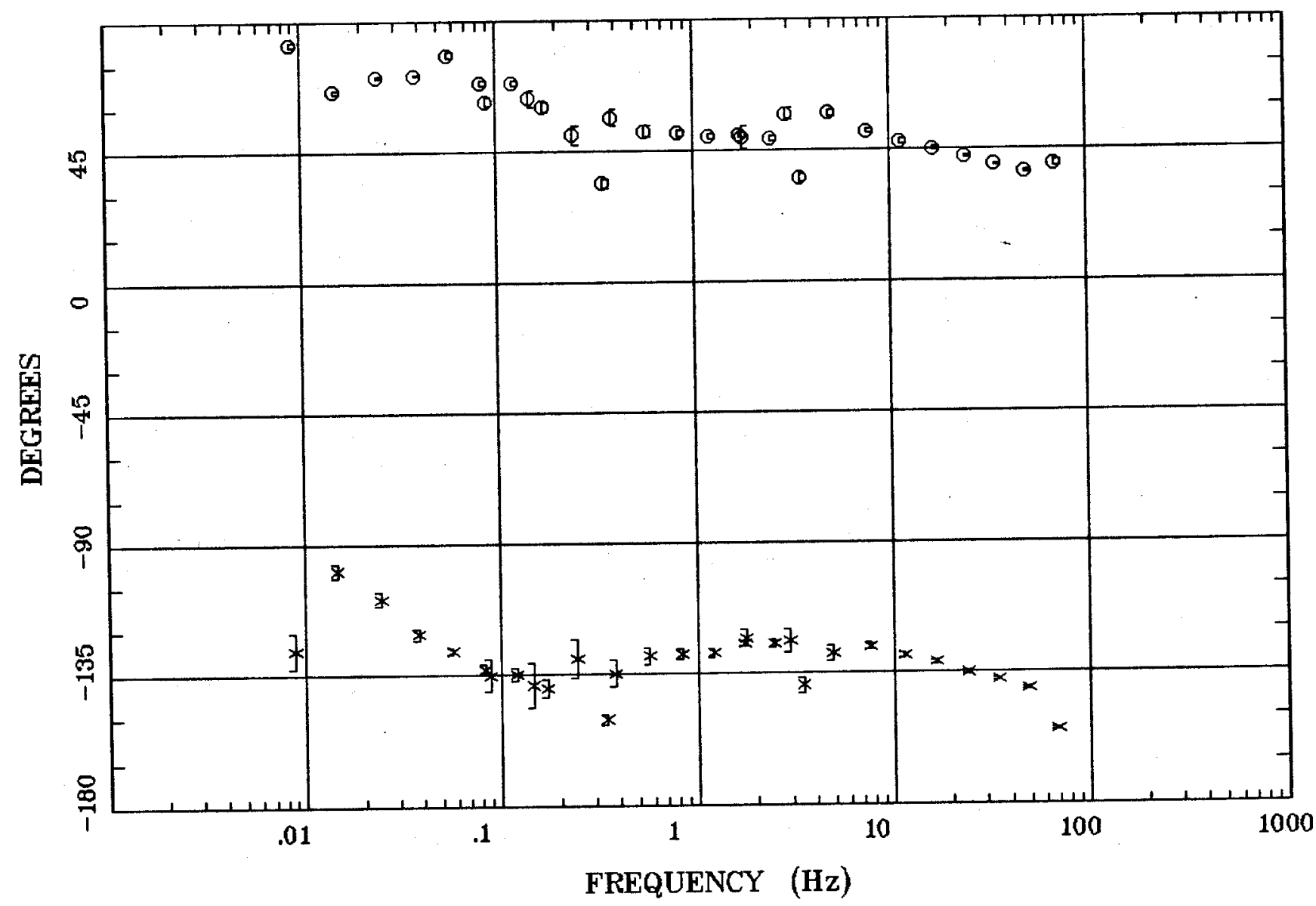

Client:

Remote: none

Acquired: 10:2 Aug 03, 2000

Survey Ca:USGS
Rotation:

Filename: hr103all.avg

Channels: Ch1 Ch2 Ch3 Ch4 Ch5 Ch3 Ch4 Plotted: 15:28 Jan 24, 2001

< EMI - ElectroMagnetic Instruments 
Station 103

ROTATION ANGLE

Crescent Valley, NV 100K

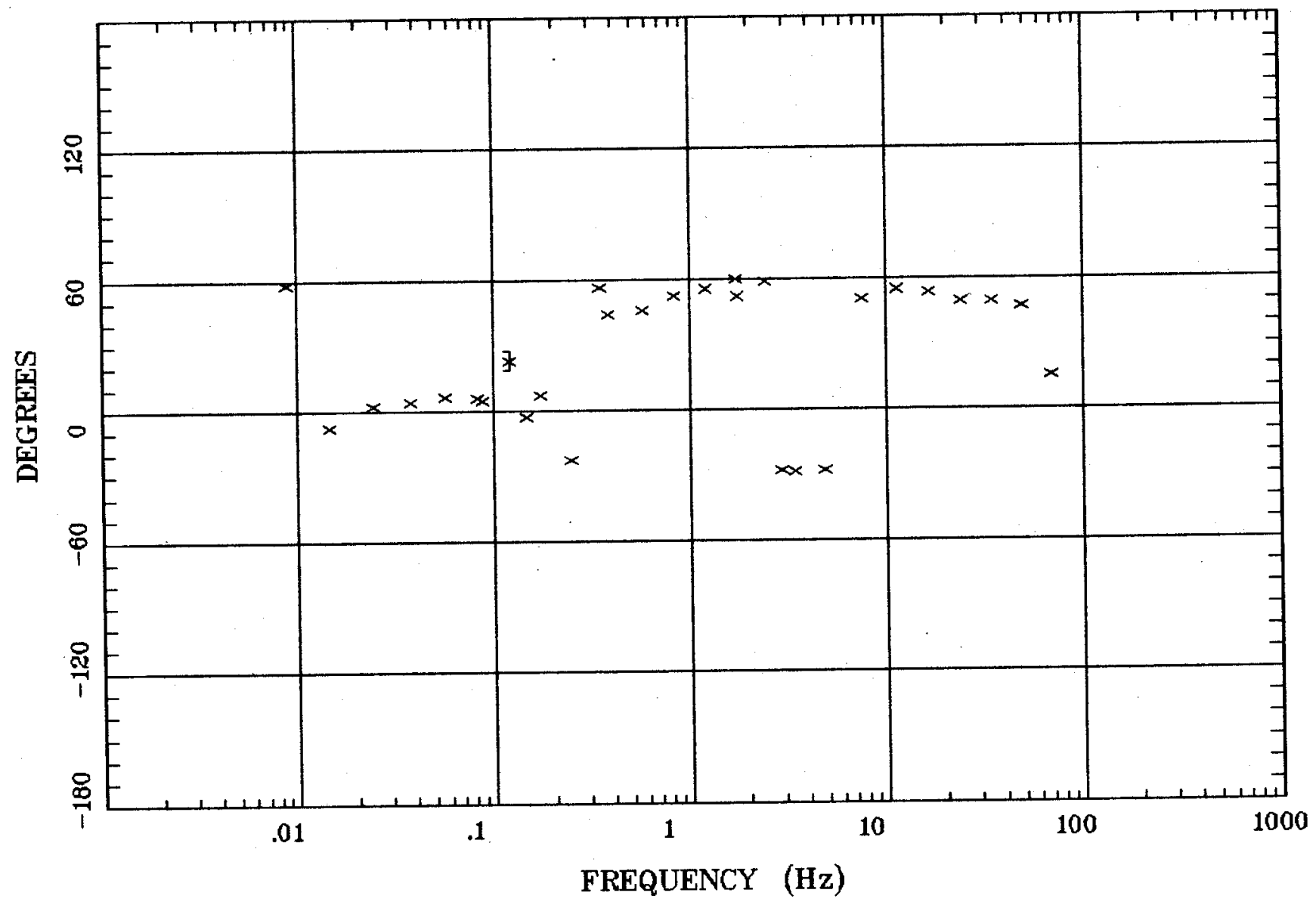

Client:

Remote: none

Acquired: 10:2 Aug 03, 2000

Survey Co:USGS

Rotation:

Filename: hr103all.avg

Channels: Ch1 Ch2 Ch3 Ch4 Ch5 Ch3 Ch4

Plotted: 15:28 Jan 24, 2001

< EMI - ElectroMagnetic Instruments > 


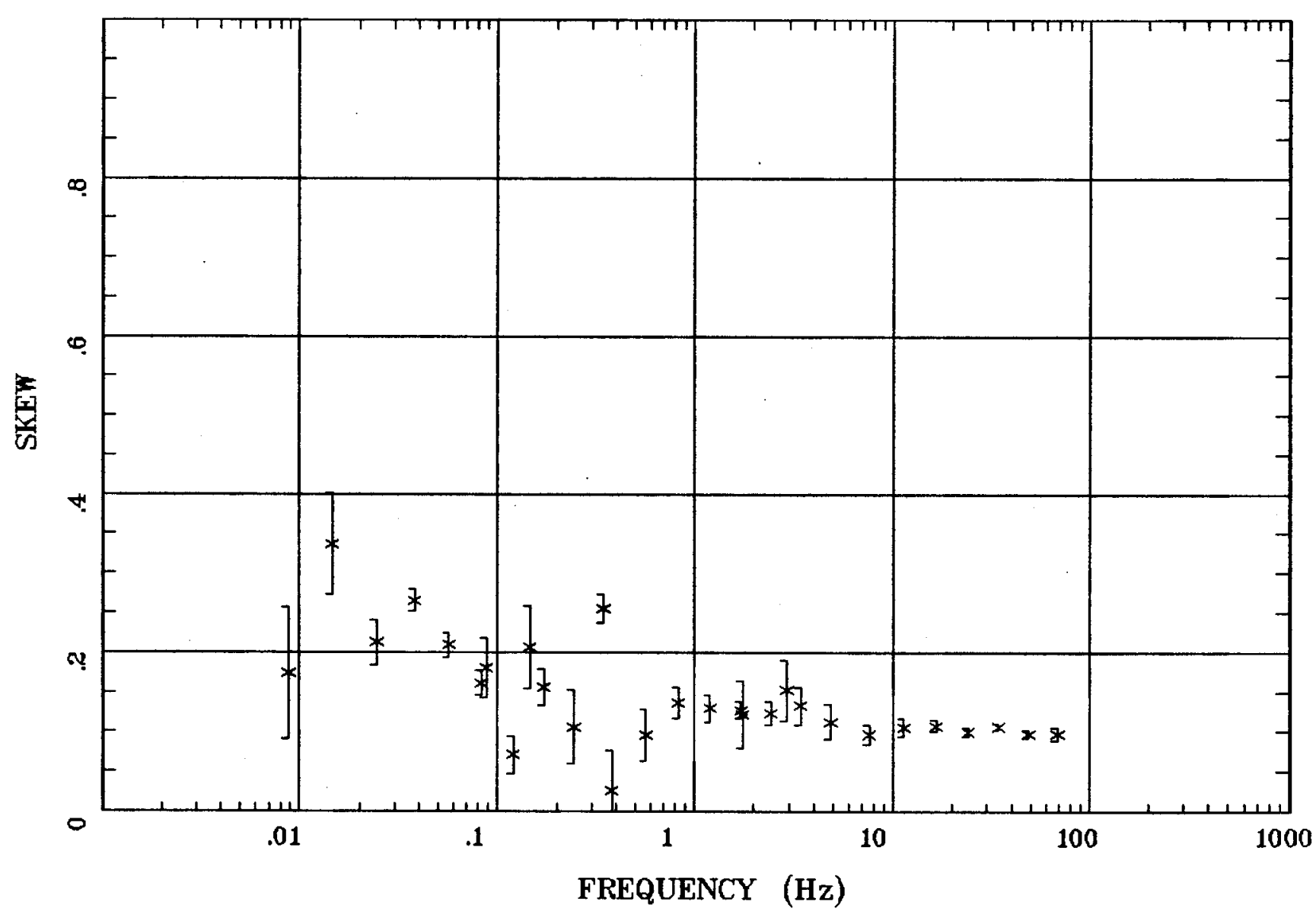

Client:

Remote: none

Acquired: 10:2 Aug 03, 2000

Survey Co:USGS
Rotation:

Filename: hr103all.avg

Channels: Ch1 Ch2 Ch3 Ch4 Ch5 Ch3 Ch4 Platted: 15:28 Jan 24, 2001

$<$ EMI - ElectroMagnetic Instruments 


\section{E MULT Coh.}

Crescent Valley, NV 100K

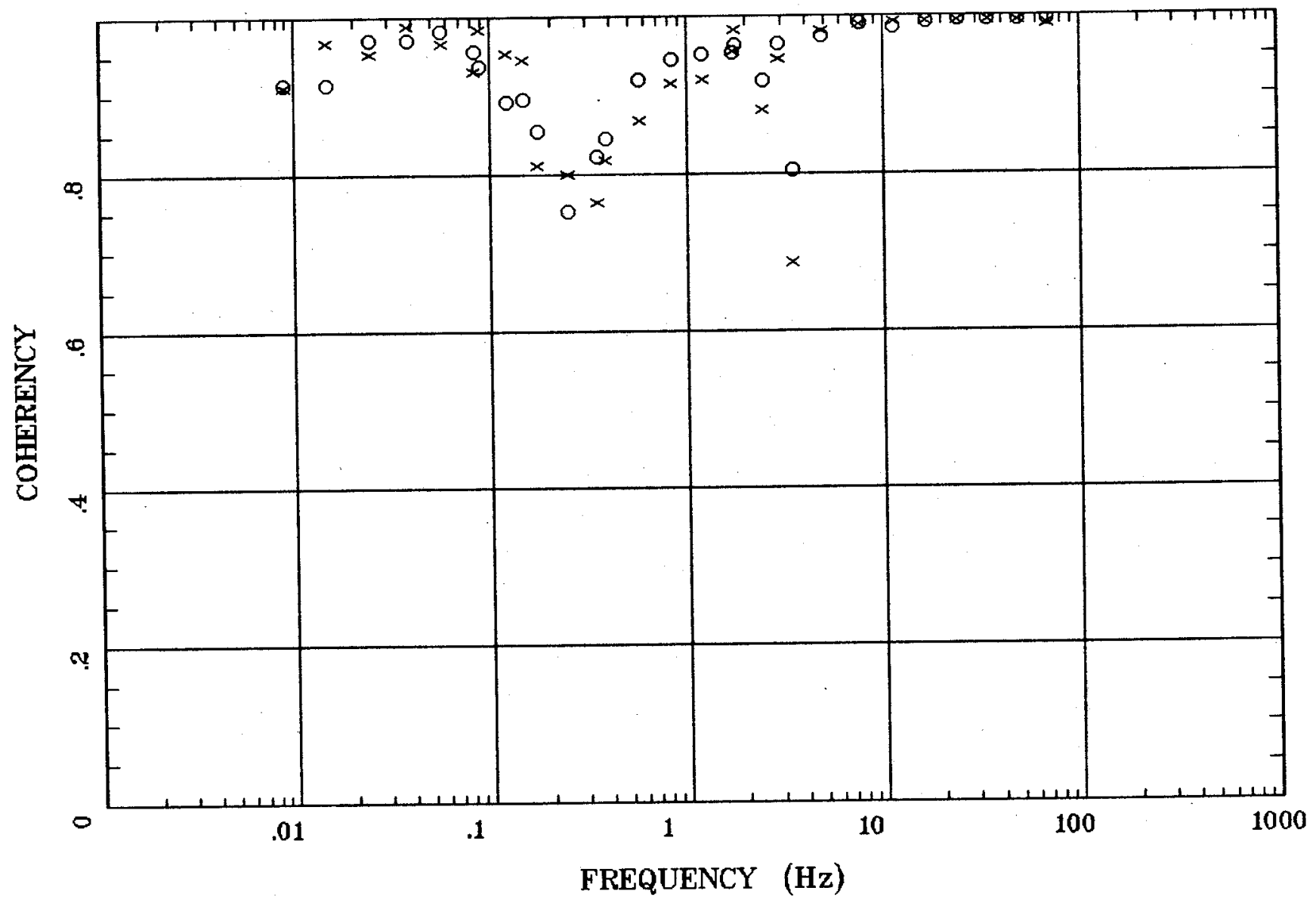

Client:

Remote: none

Acquired: 10:2 Aug 03, 2000 Survey Co:USGS
Rotation:

Filename: hr103all.avg

Channels: Ch1 Ch2 Ch3 Ch4 Ch5 Ch3 Ch4

Plotted: 15:28 Jan 24, 2001

< EMI - ElectroMagnetic Instruments > 


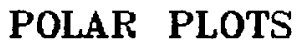

Crescent Valley, NV 100K

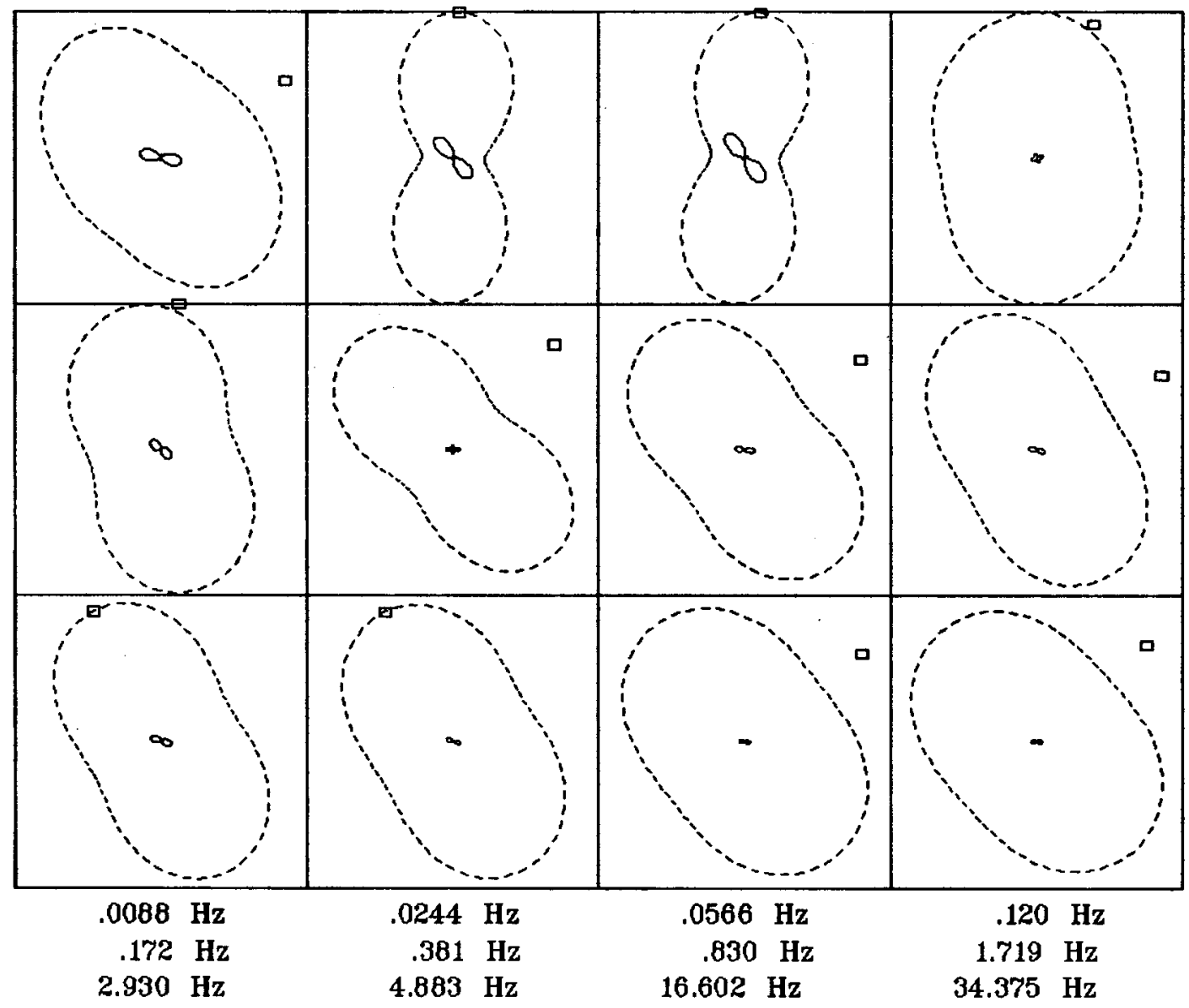

Client:

Remote: none

Acquired: 10:2 Aug 03, 2000

Survey Co:USGS
Rotation:

Filename: hr103all.avg

Channels: Ch1 Ch2 Ch3 Ch4 Ch5 Ch3 Ch4 Plotted: 15:28 Jan 24, 2001

$<$ EMI - ElectroMagnetic Instruments 


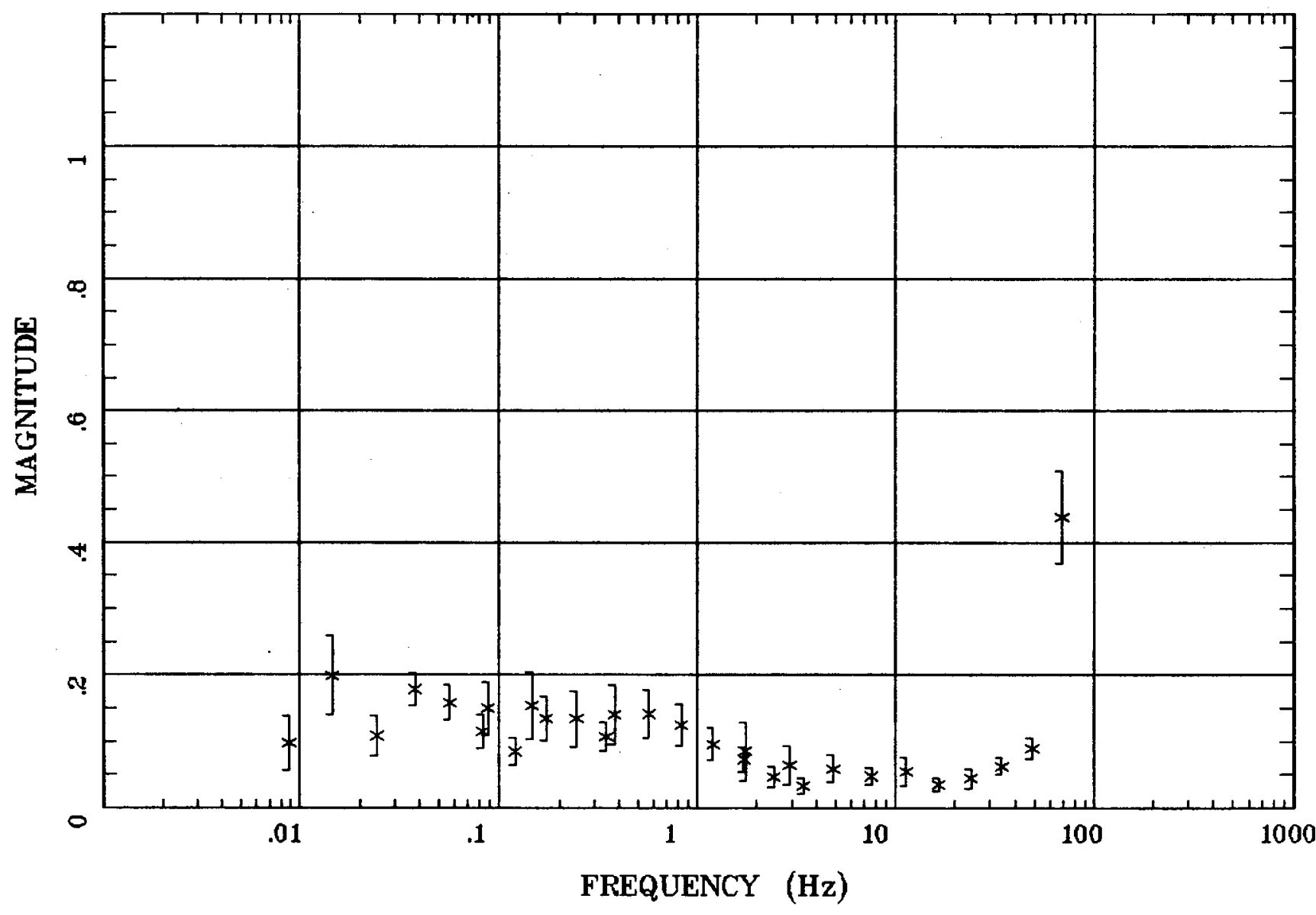

Client:

Remote: none

Acquired: 10:2 Aug 03, 2000 Survey Co:USGS
Rotation:

Filename: hr103all.avg

Channels: Ch1 Ch2 Ch3 Ch4 Ch5 Ch3 Ch4 Platted: 15:28 Jan 24, 2001

< EMI - ElectroMagnetic Instruments 


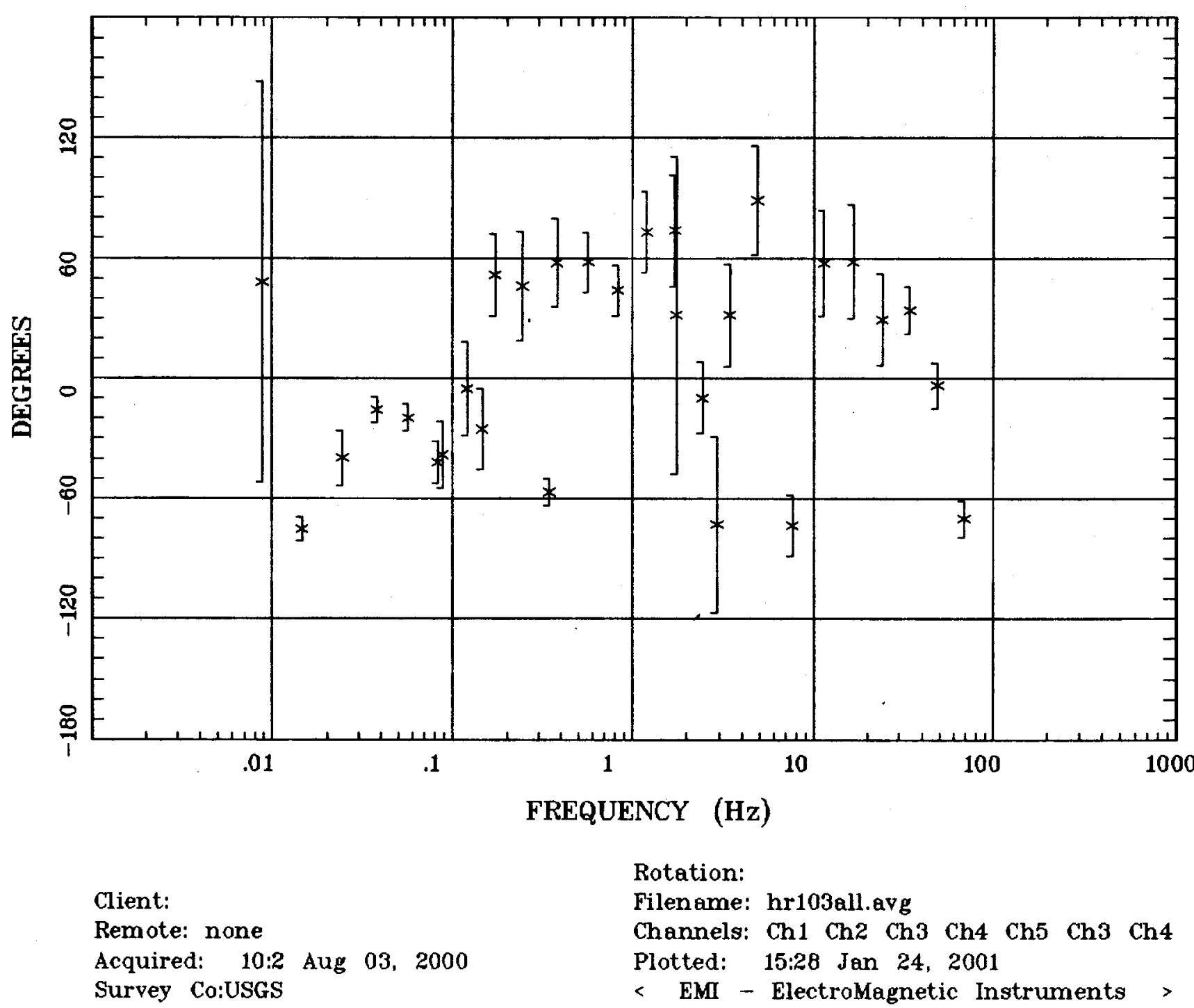


Station 103

HzHx.x Coh HzHy.o Crescent Valley, NV 100K

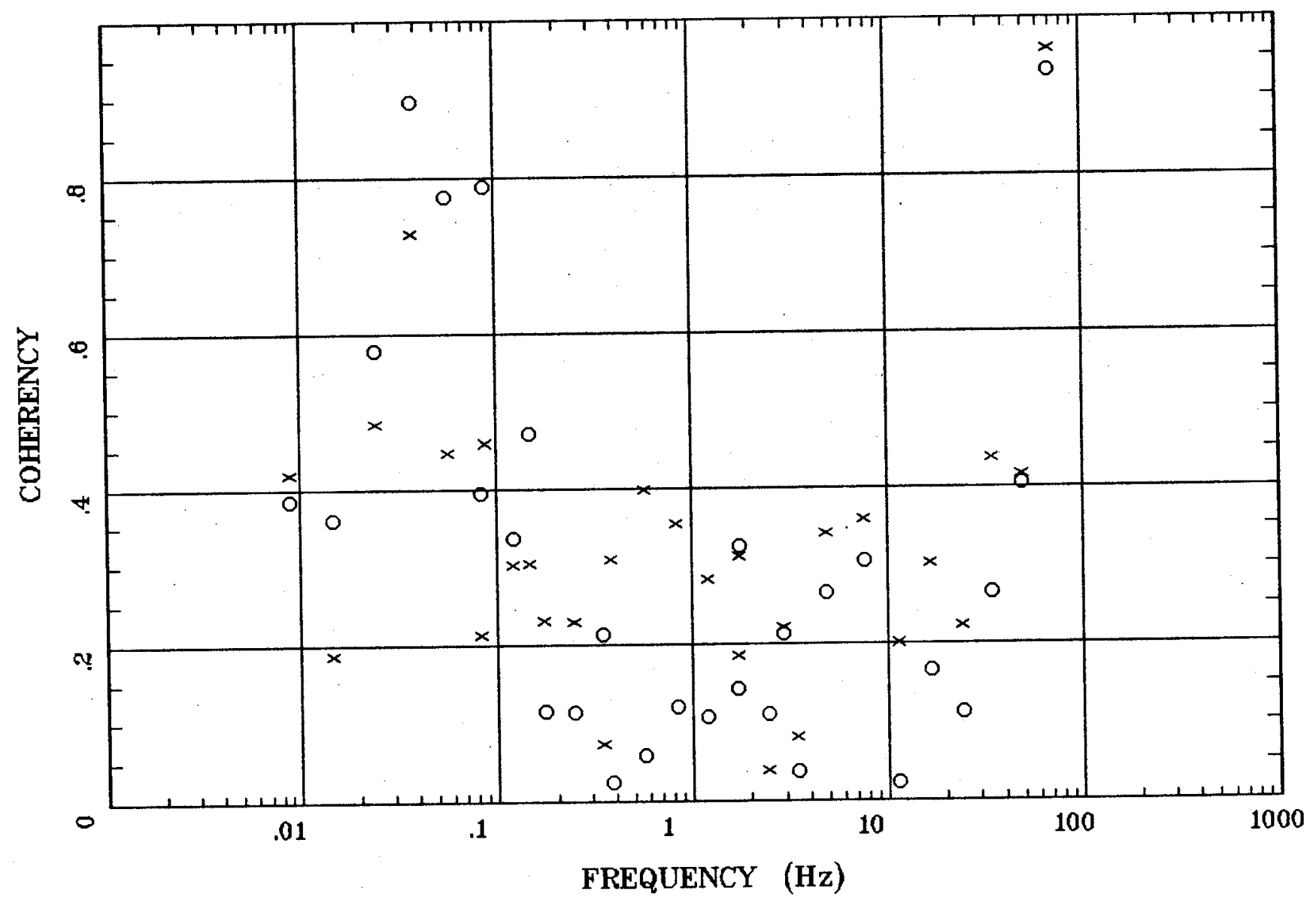

Rotation:

Client:

Filename: hr103all.avg

Remote: none

Acquired: 10:2 Aug 03, 2000

Channels: Ch1 Ch2 Ch3 Ch4 Ch5 Ch3 Ch4

Platted: 15:28 Jan 24, 2001

Survey Co:USGS

< EMI - ElectroMagnetic Instruments 


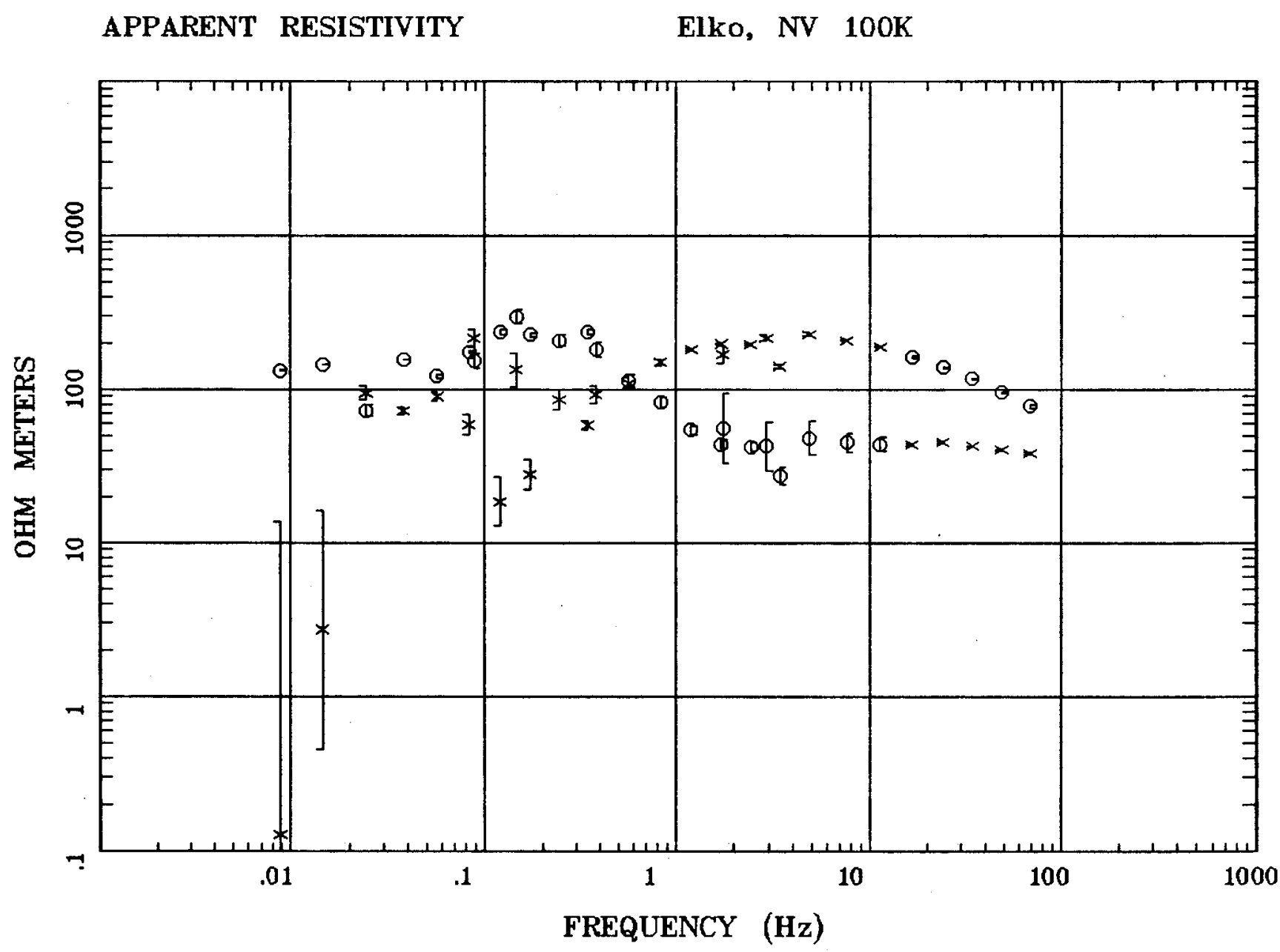

Client:

Remote: none Acquired: 14:4 Aug 03, 2000 Survey Co:USGS
Rotation:

Filename: hr104all.avg

Channels: Ch1 Ch2 Ch3 Ch4 Ch5 Ch3 Ch4 Plotted: 15:28 Jan 24, 2001

$<$ EMI - ElectroMagnetic Instruments 
Station 104

IMPEDANCE PHASE

Elko, NV 100K

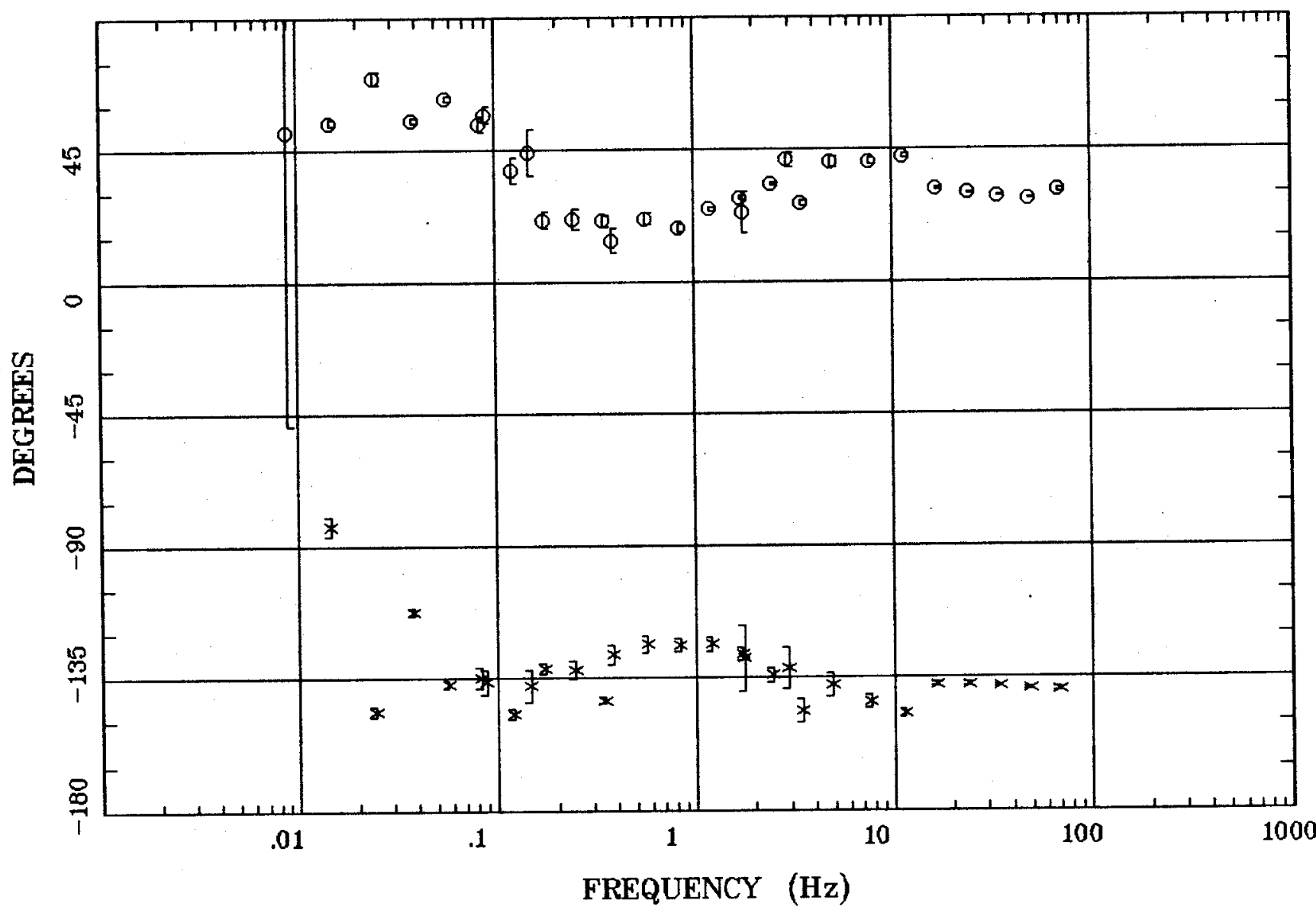

\section{Client:}

Remote: none

Acquired: 14:4 Aug 03, 2000

Survey Co:USGS
Rotation:

Filen ame: hr104allavg

Channels: Ch1 Ch2 Ch3 Ch4 Ch5 Ch3 Ch4

Plotted: 15:28 Jan 24, 2001

< EMI - ElectroMagnetic Instruments > 


\section{Station 104}

ROTATION ANGLE

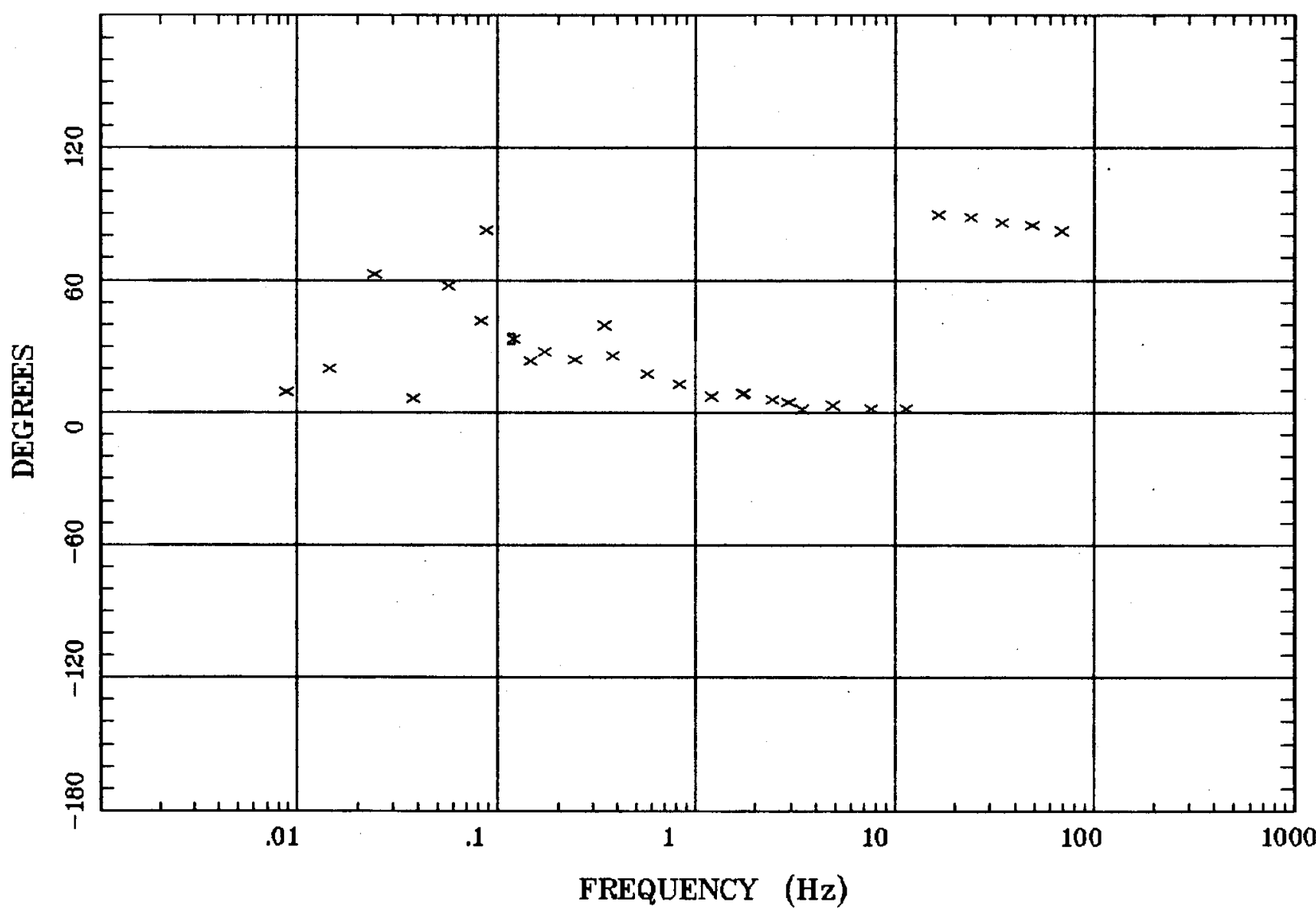

Client:

Remote: none

Acquired: 14:4 Aug 03, 2000 Survey Co:USGS
Rotation:

Filename: hr104all.avg

Channels: Ch1 Ch2 Ch3 Ch4 Ch5 Ch3 Ch4

Platted: 15:28 Jan 24, 2001
< EMI - ElectroMagnetic Instruments 
IMPEDANCE SKEW Elko, NV 100K

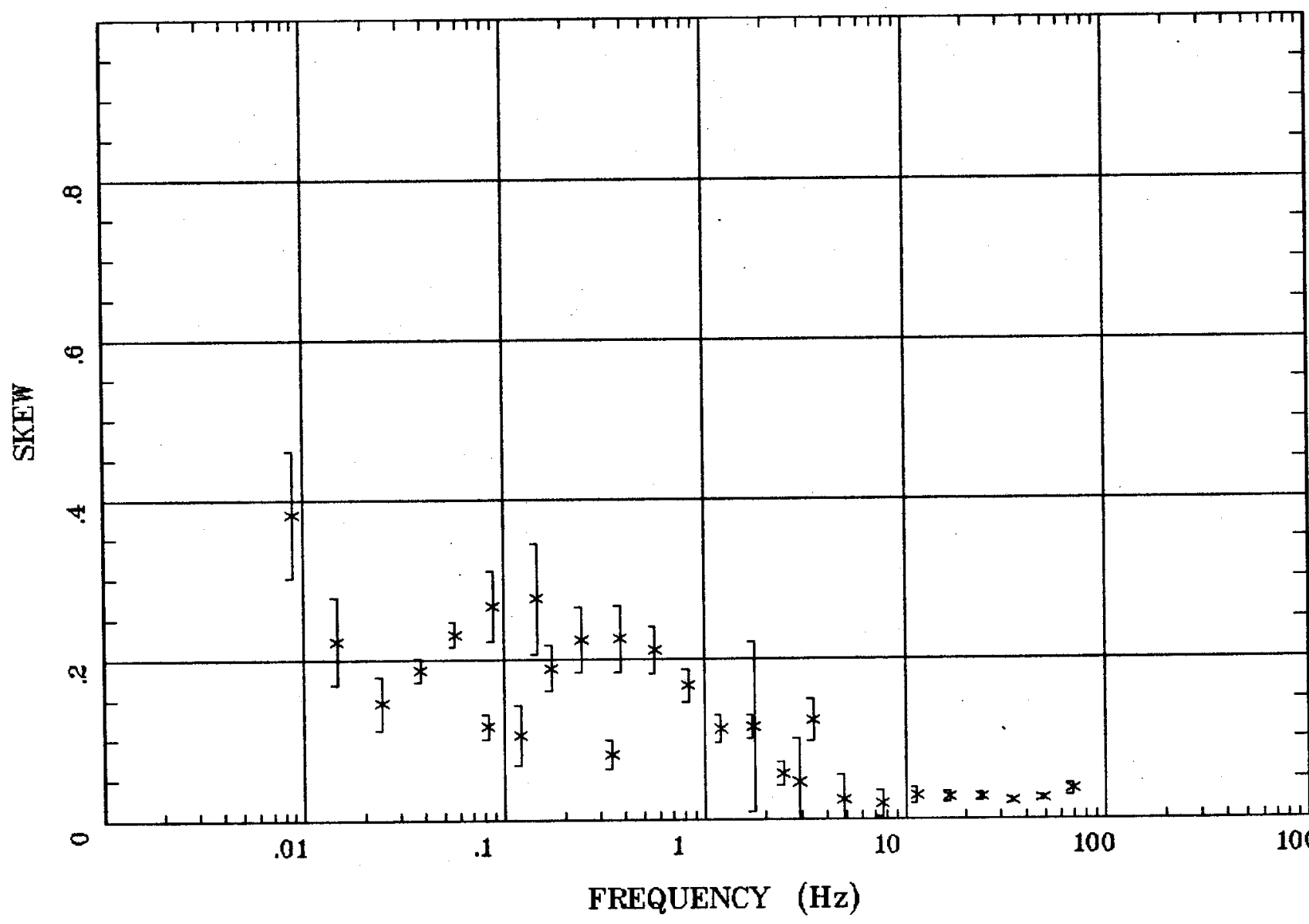

Client:

Remote: none

Acquired: 14:4 Aug 03, 2000

Survey Co:USGS
Rotation:

Filename: hr104all.avg

Channels: Ch1 Ch2 Ch3 Ch4 Ch5 Ch3 Ch4

Plotted: 15:28 Jan 24, 2001

$<$ EMI - ElectroMagnetic Instruments 
E MULT Coh.

Elko, NV 100K

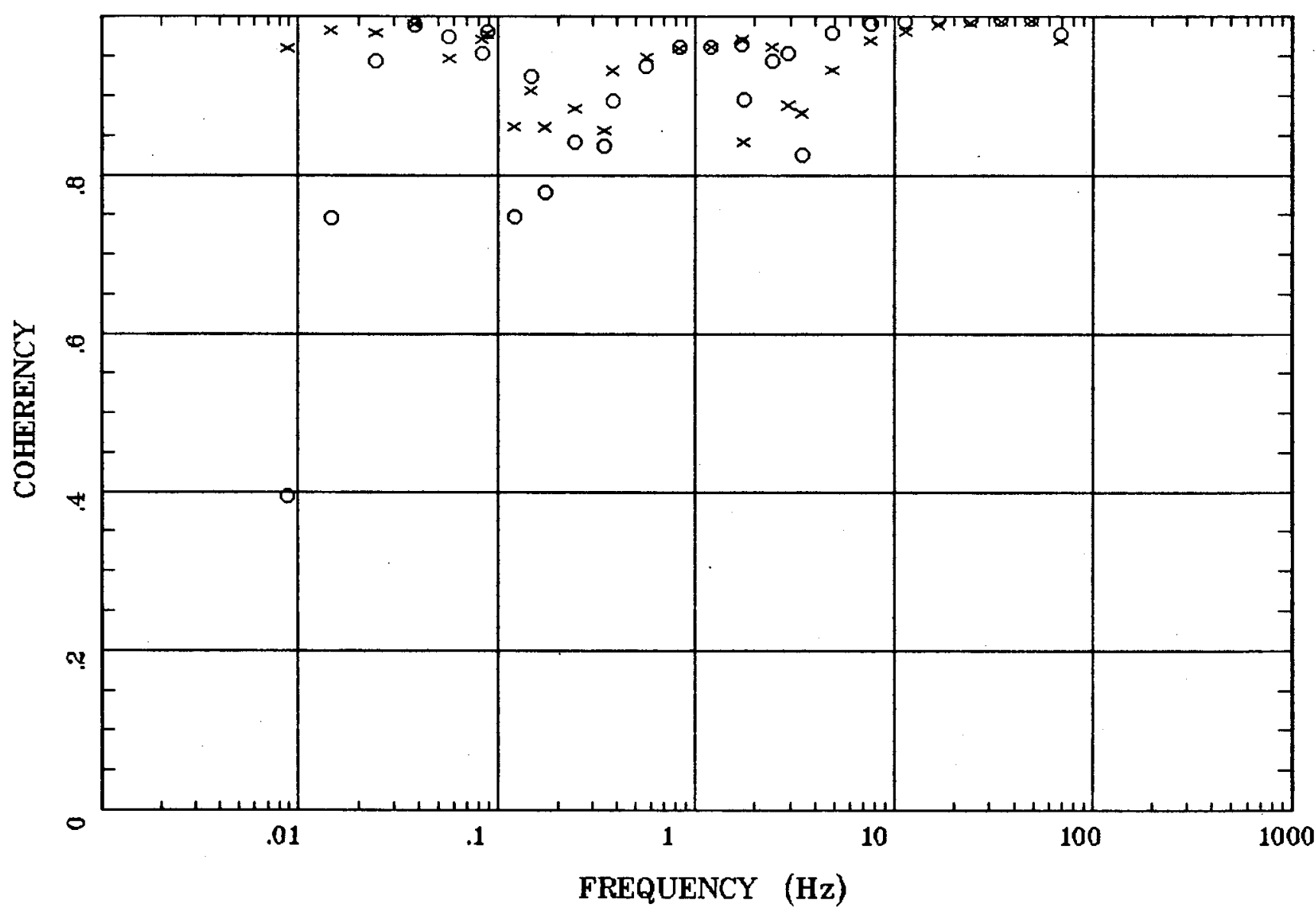

Client:

Remote: none

Acquired: 14:4 Aug 03, 2000 Survey Co:USGS
Rotation:

Filename: hr104all.avg

Channels: Ch1 Ch2 Ch3 Ch4 Ch5 Ch3 Ch4

Plotted: 15:28 Jan 24, 2001

< EMI - ElectroMagnetic Instruments > 


\section{POLAR PLOTS}

\section{Elko, NV 100K}

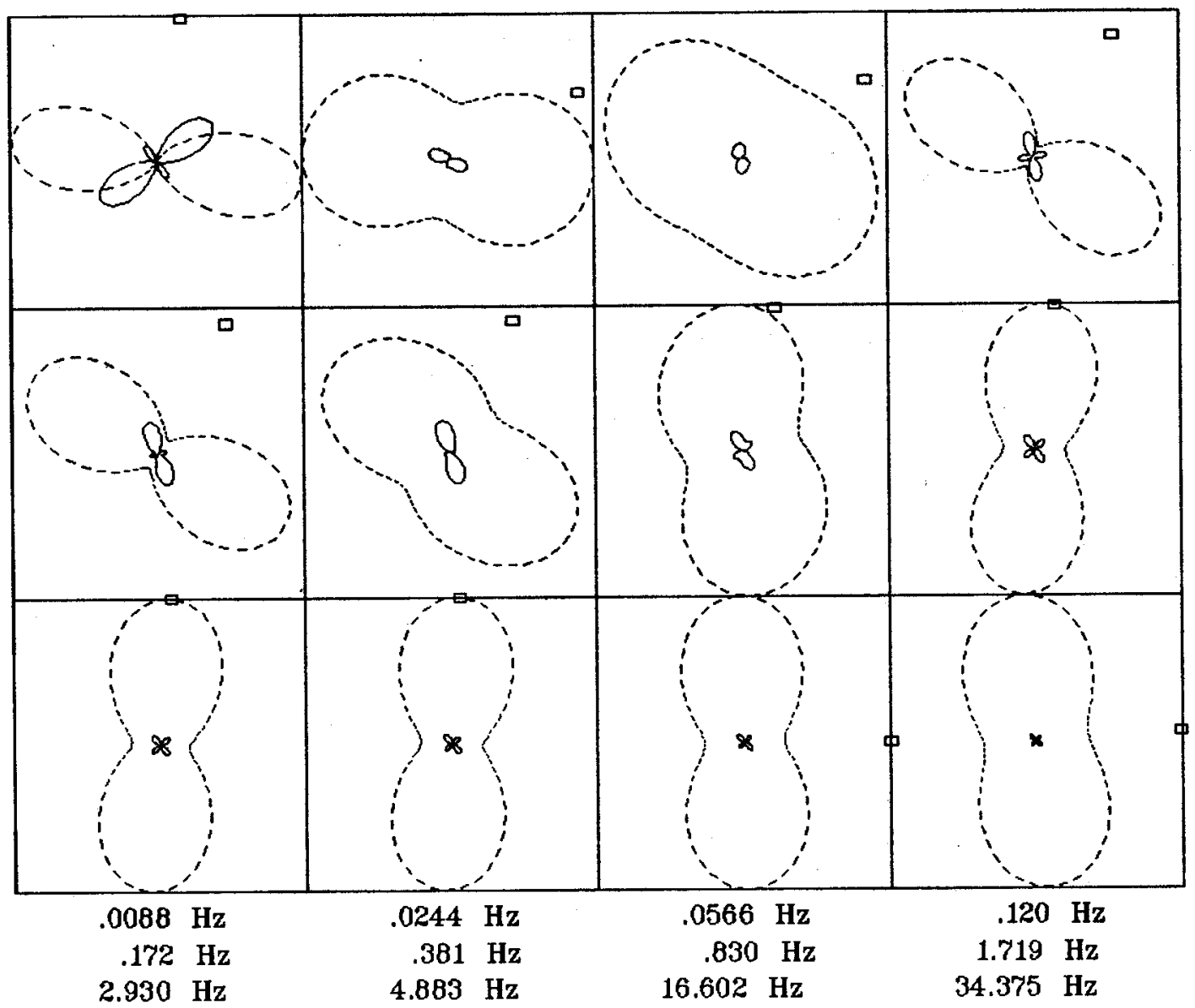

Rotation:

Client:

Remote: none

Acquired: 14:4 Aug 03, 2000 Survey Co:USGS
Filename: hr104all.avg

Channels: Ch1 Ch2 Ch3 Ch4 Ch5 Ch3 Ch4

Plotted: 15:28 Jan 24, 2001

$<$ EMI - ElectroMagnetic Instruments > 
TIPPER MAGNITUDE

Elko, NV 100K

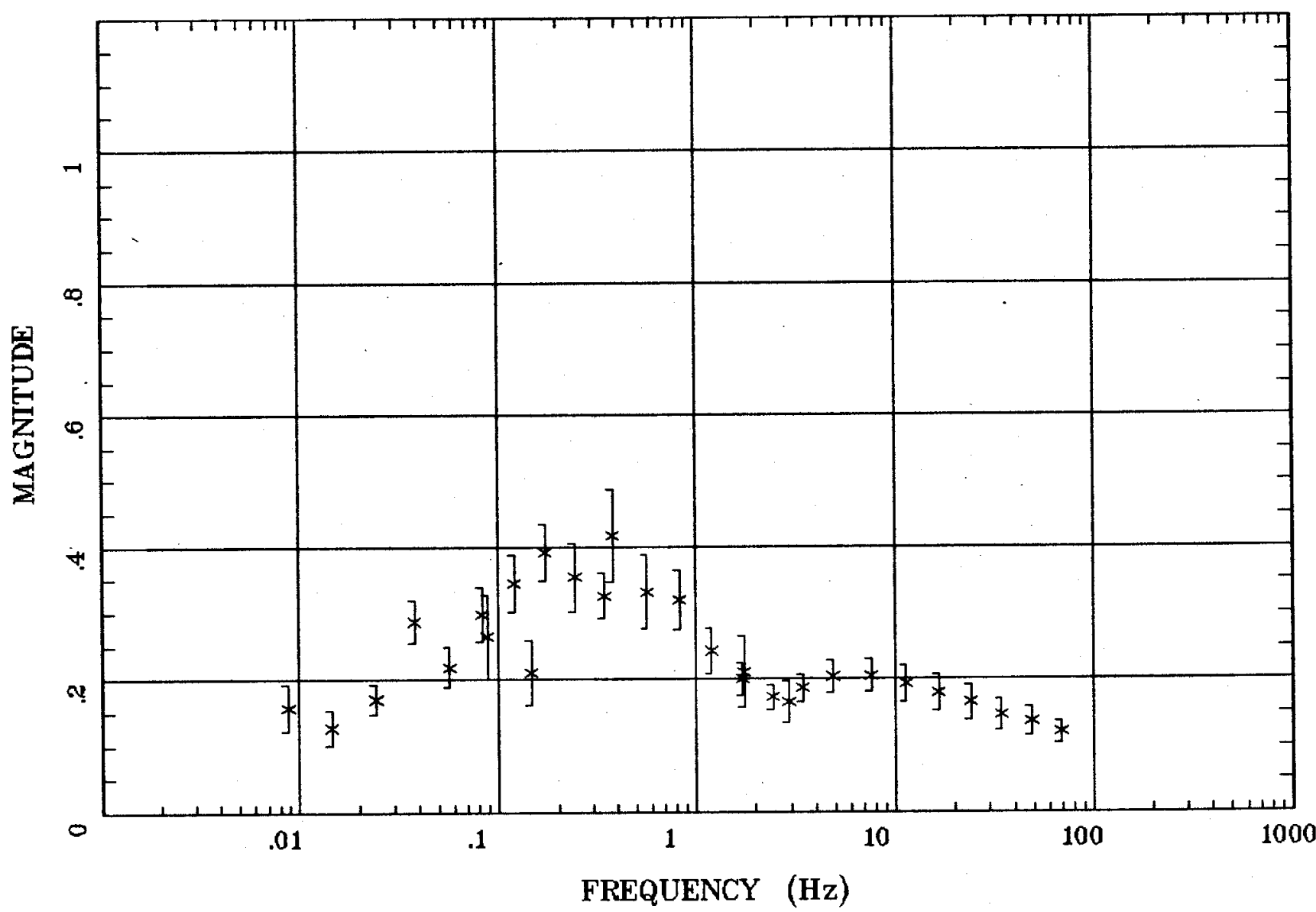

Client:

Remote: none

Acquired: 14:4 Aug 03, 2000

Survey Co:USGS
Rotation:

Filename: hr104all.avg

Channels: Ch1 Ch2 Ch3 Ch4 Ch5 Ch3 Ch4

Plotted: 15:28 Jan 24, 2001

< EMI - ElectroMagnetic Instruments > 


\section{Station 104}

TIPPER STRIKE

Elko, NV 100K

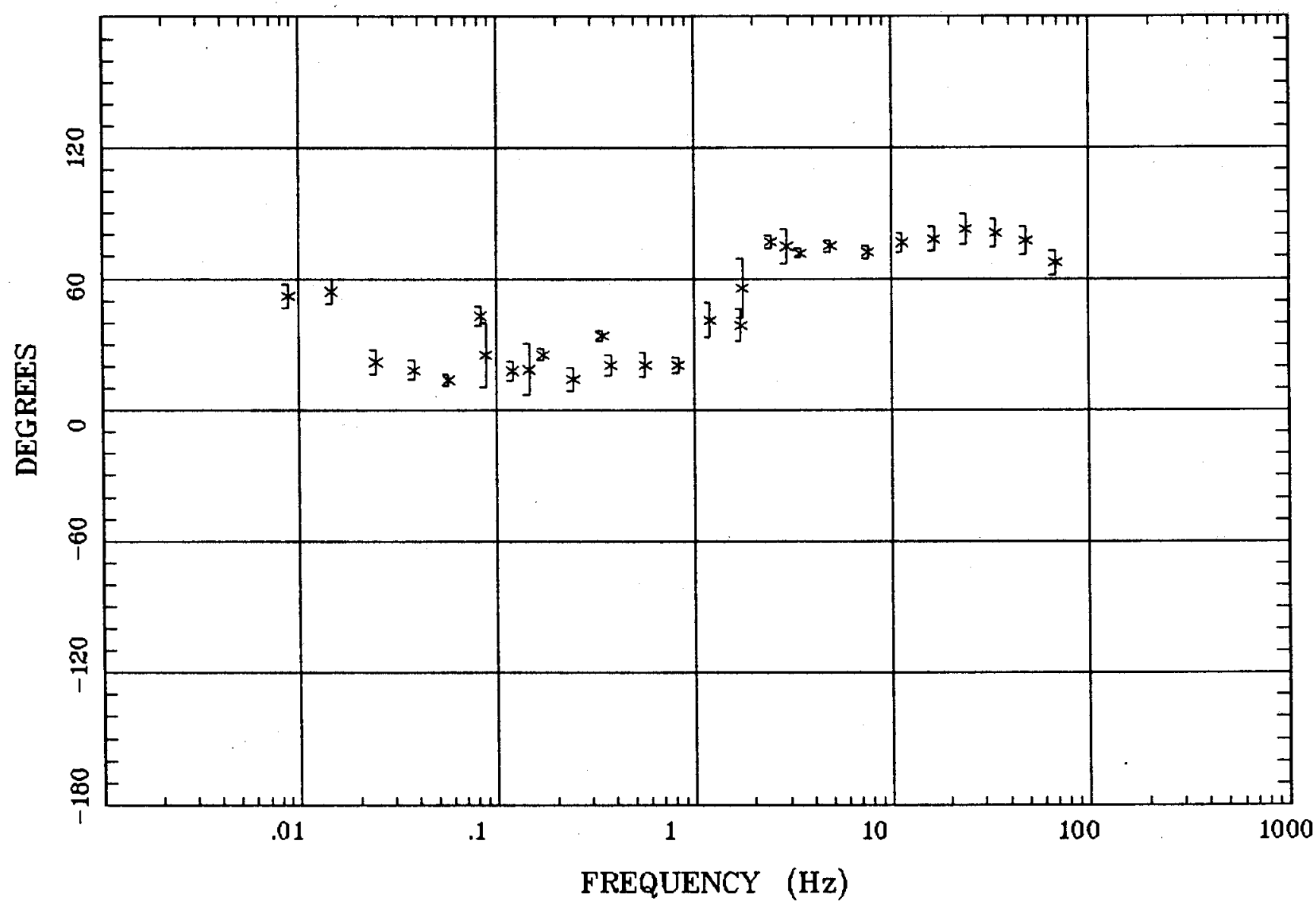

Client:

Remote: none

Acquired: 14:4 Aug 03, 2000

Survey Co:USGS
Rotation:

Filename: hr104all.avg

Channels: Ch1 Ch2 Ch3 Ch4 Ch5 Ch3 Ch4

Plotted: 15:28 Jan 24, 2001

< EMI - ElectroMagnetic Instruments > 


\section{HzHx.x Coh HzHy.o}

Elko, NV 100K

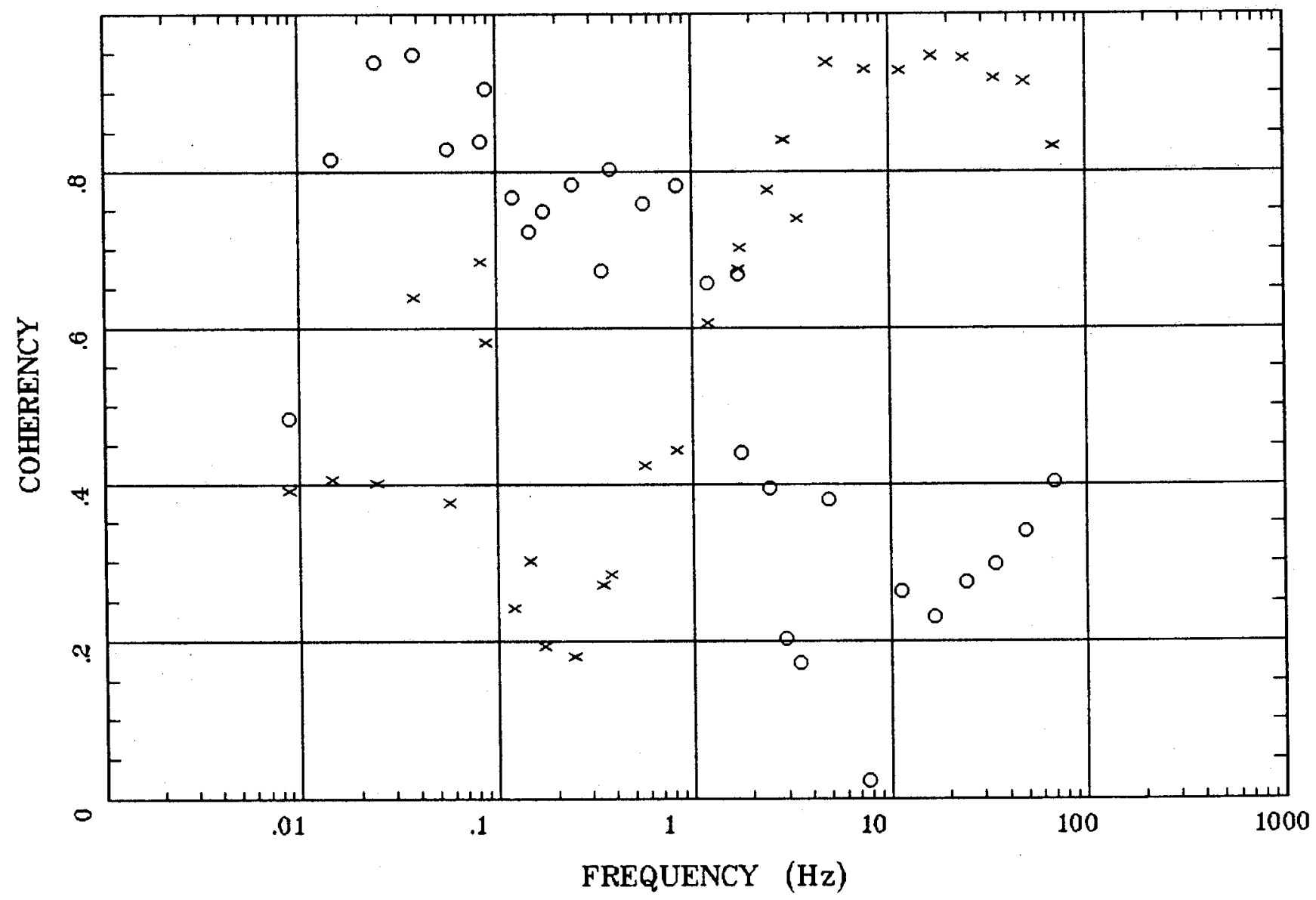

Client:

Remote: none

Acquired: 14:4 Aug 03, 2000 Survey Co:USGS
Rotation:

Filename: hr104all.avg

Channels: Ch1 Ch2 Ch3 Ch4 Ch5 Ch3 Ch4 Plotted: 15:28 Jan 24, 2001

$<$ EMI - ElectroMagnetic Instruments 


\section{APPARENT RESISTIVITY}

Indian Creek, Shoshone Mts.

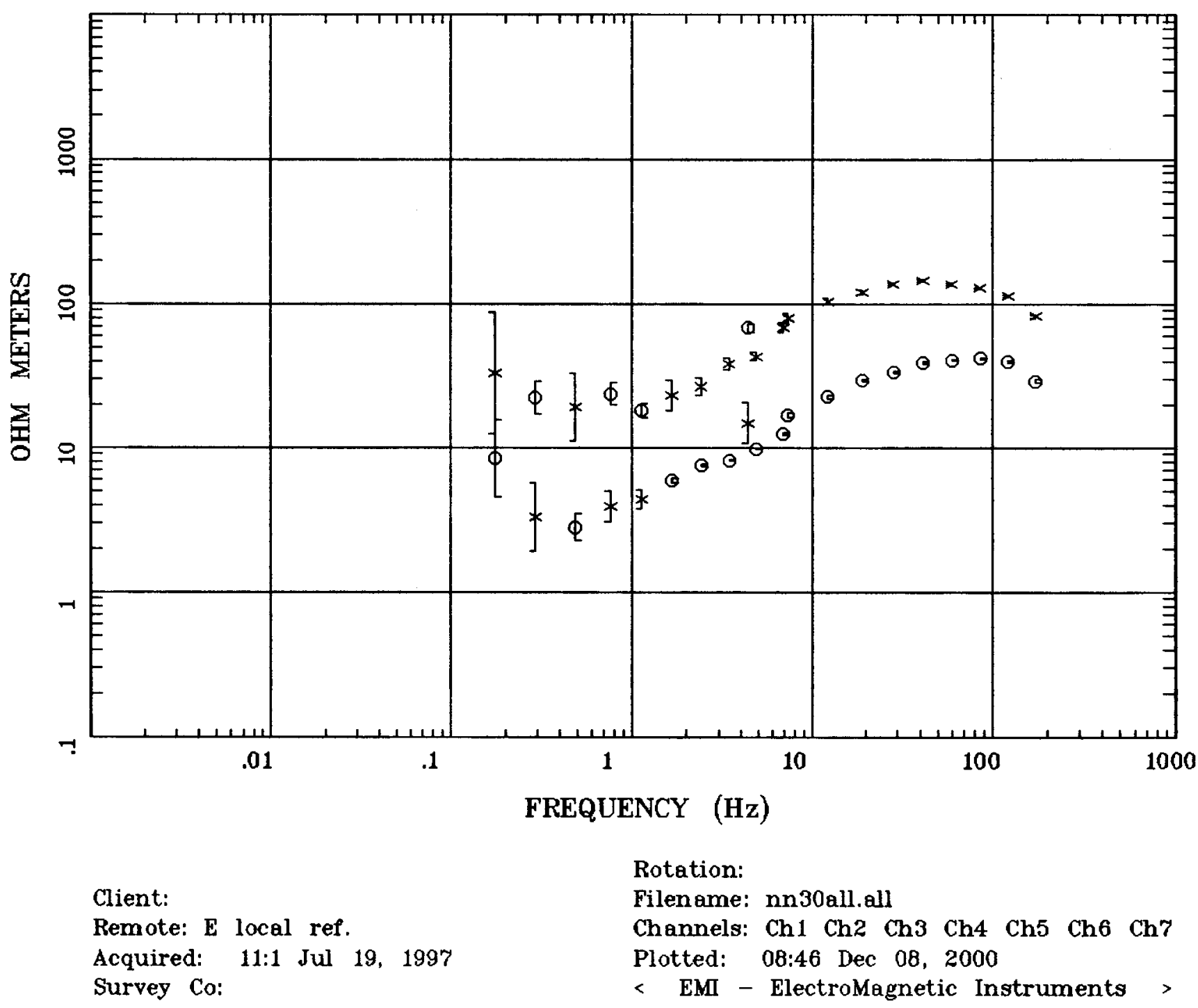




\section{Station 30}

Indian Creek, Shoshone Mts.

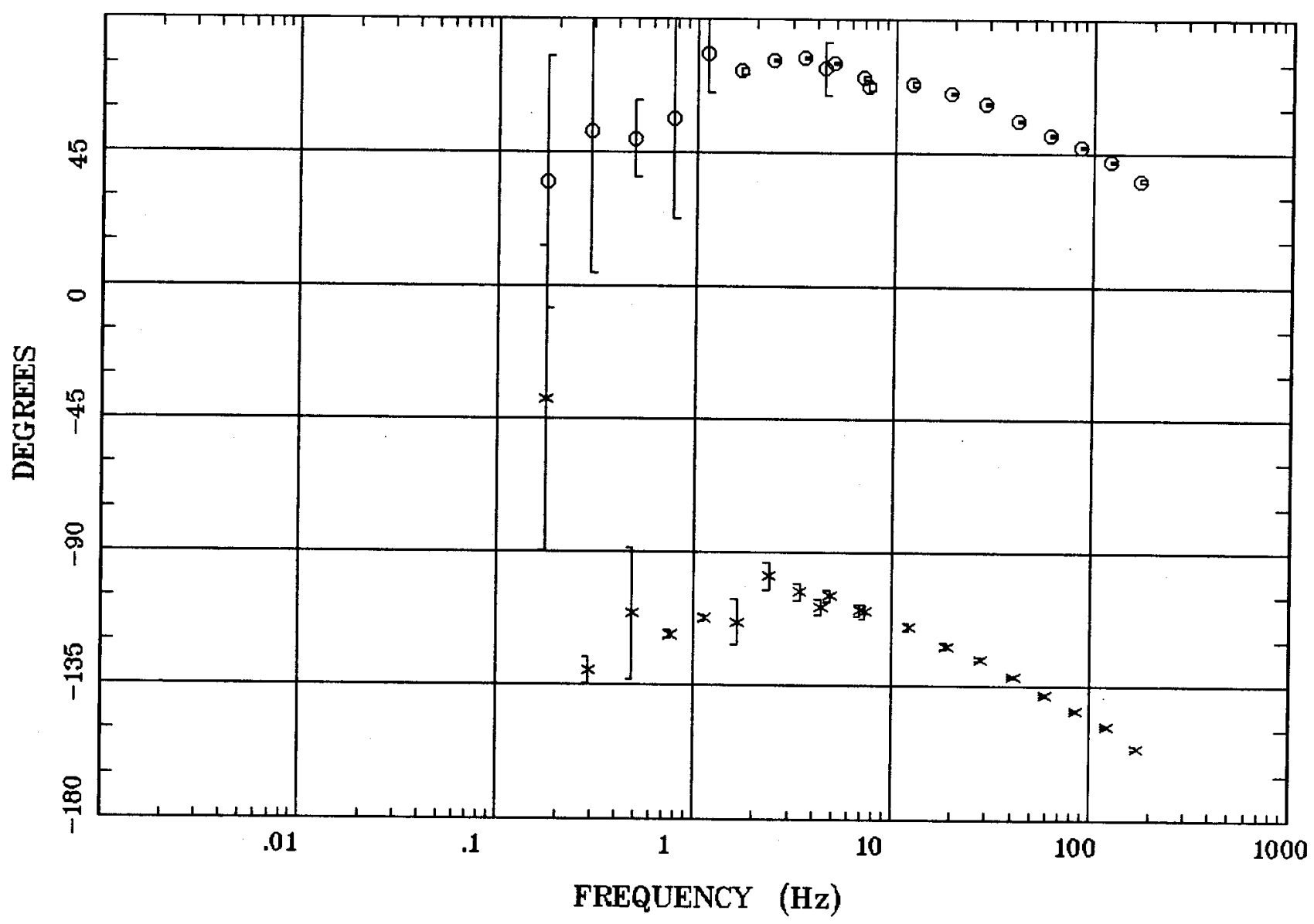

Client:

Remote: E local ref.

Acquired: 11:1 Jul 19, 1997

Survey Co:
Rotation:

Filename: nn30all.all

Channels: Ch1 Ch2 Ch3 Ch4 Ch5 Ch6 Ch7

Plotted: 10:50 Jan 25, 2001

$<$ EMI - ElectroMagnetic Instruments 
Station 30

ROTATION ANGLE

Indian Creek, Shoshone Mts.

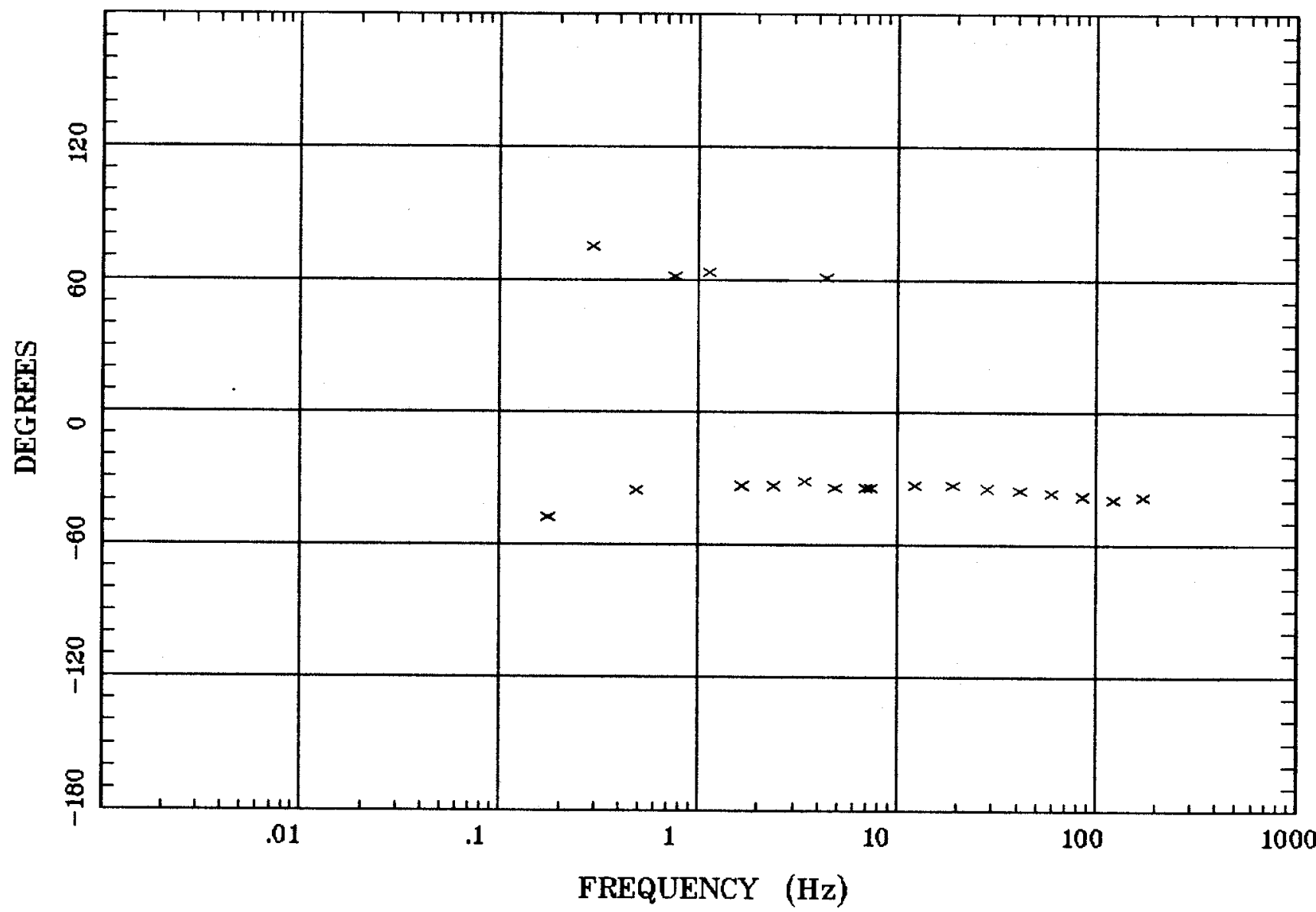

Client:

Remote: E local ref.

Acquired: 11:1 Jul 19, 1997

Survey Co:

Rotation:

Filename: nn30all.all

Channels: Ch1 Ch2 Ch3 Ch4 Ch5 Ch6 Ch7

Plotted: 08:46 Dec 08, 2000

< EMI - ElectroMagnetic Instruments 


\section{Station 30}

IMPEDANCE SKEW

Indian Creek, Shoshone Mts.

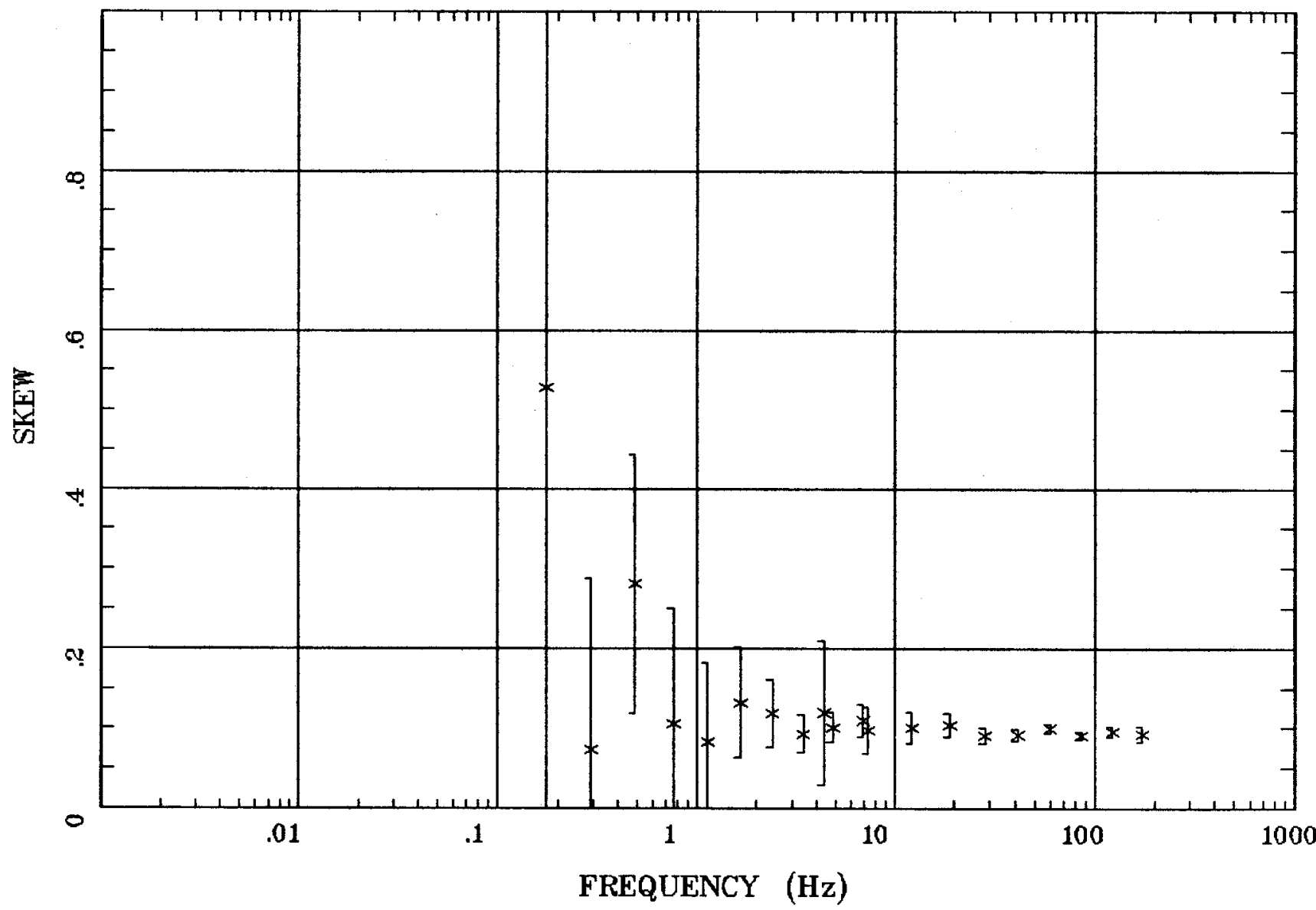

Client:

Remote: E local ref.

Acquired: 11:1 Jul 19, 1997

Survey Co:

Rotation:

Filename: nn30all.all

Channels: Ch1 Ch2 Ch3 Ch4 Ch5 Ch6 Ch7

Plotted: 08:46 Dec 08, 2000

$<$ EMI - ElectroMagnetic Instruments 
Station 30

E MULT Coh.

Indian Creek, Shoshone Mts.

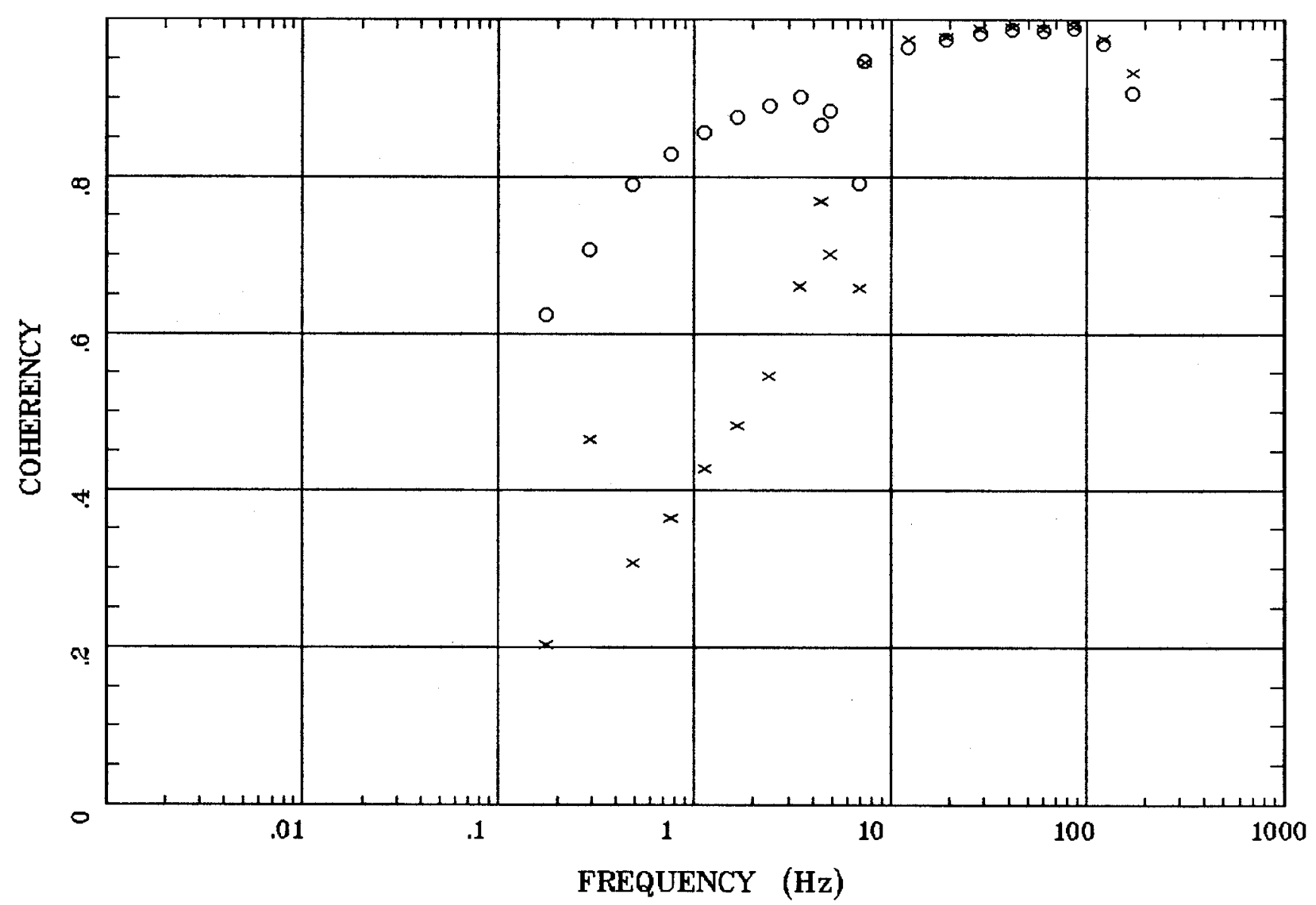

Client:

Remote: E local ref.

Acquired: 11:1 Jul 19, 1997

Survey Co:
Rotation:

Filename: nn30all.all

Channels: Ch1 Ch2 Ch3 Ch4 Ch5 Ch6 Ch7

Plotted: 08:46 Dec 08, 2000

< EMI - ElectroMagnetic Instruments > 
Indian Creek, Shoshone Mts.

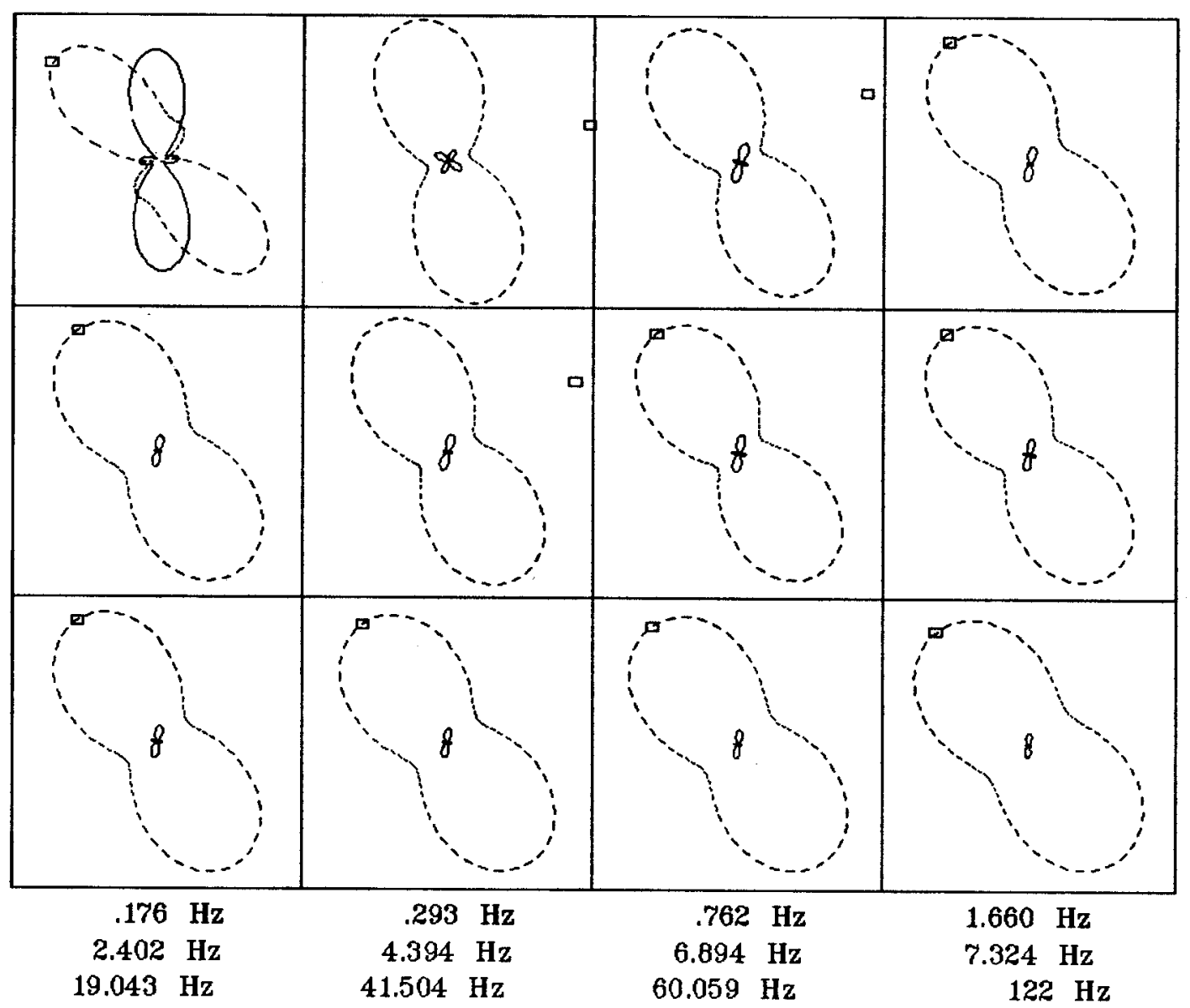

Client:

Remote: E local ref.

Acquired: 11:1 Jul 19, 1997

Survey Co:
Rotation:

Filename: nn30all.all

Channels: Ch1 Ch2 Ch3 Ch4 Ch5 Ch6 Ch7

Plotted: 08:46 Dec 08, 2000

< EMI - ElectroMagnetic Instruments > 


\section{Station 30}

TIPPER MAGNITUDE

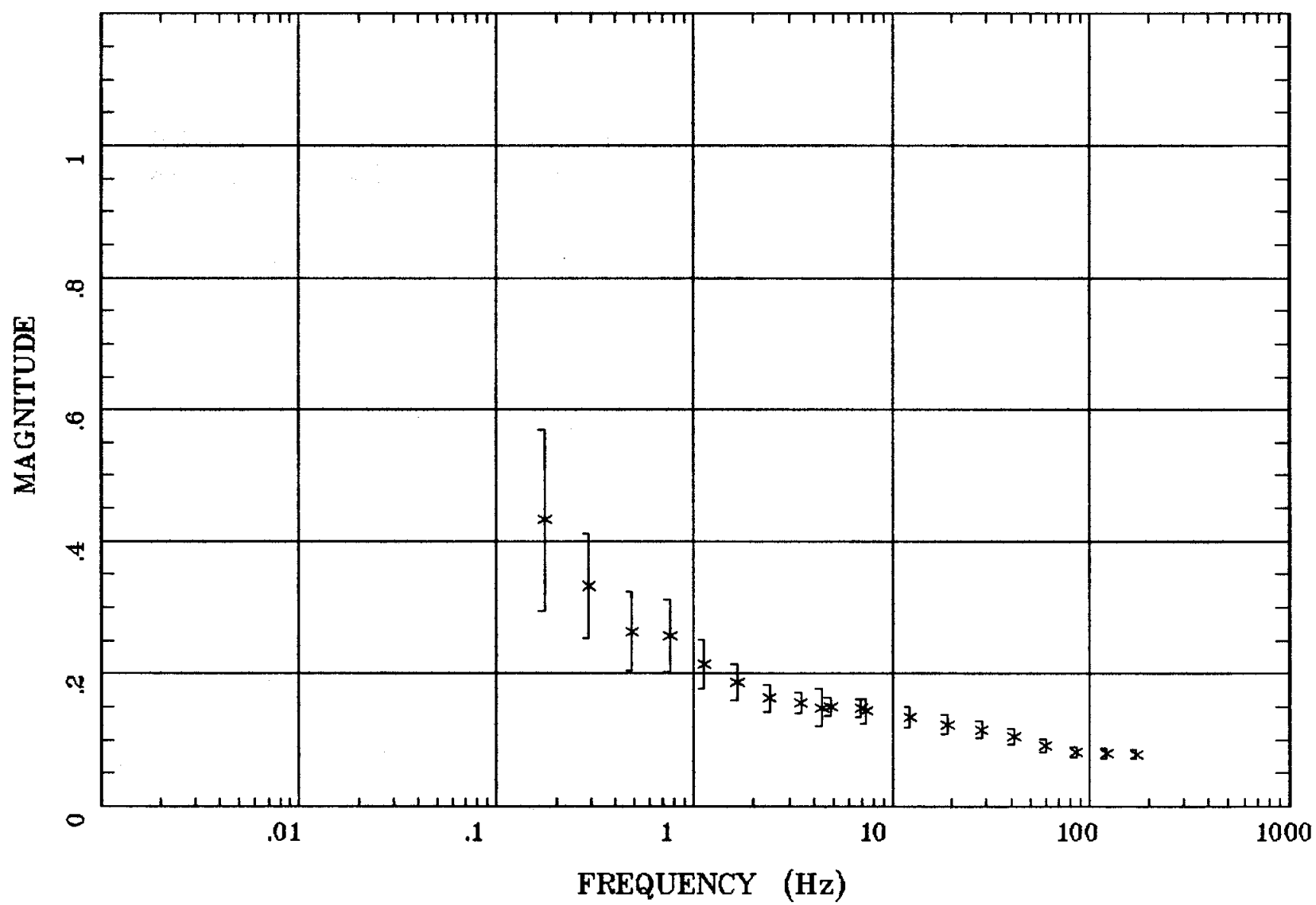

Client:

Remote: E local ref.

Acquired: 11:1 Jul 19, 1997

Survey Co:
Indian Creek, Shoshone Mts.

$$
\text { : }
$$

Rotation:

Filename: nn30all.all

Channels: Ch1 Ch2 Ch3 Ch4 Ch5 ch6 Ch7

Plotted: 08:46 Dec 08, 2000

< EMI - ElectroMagnetic Instruments > 
Indian Creek, Shoshone Mts.

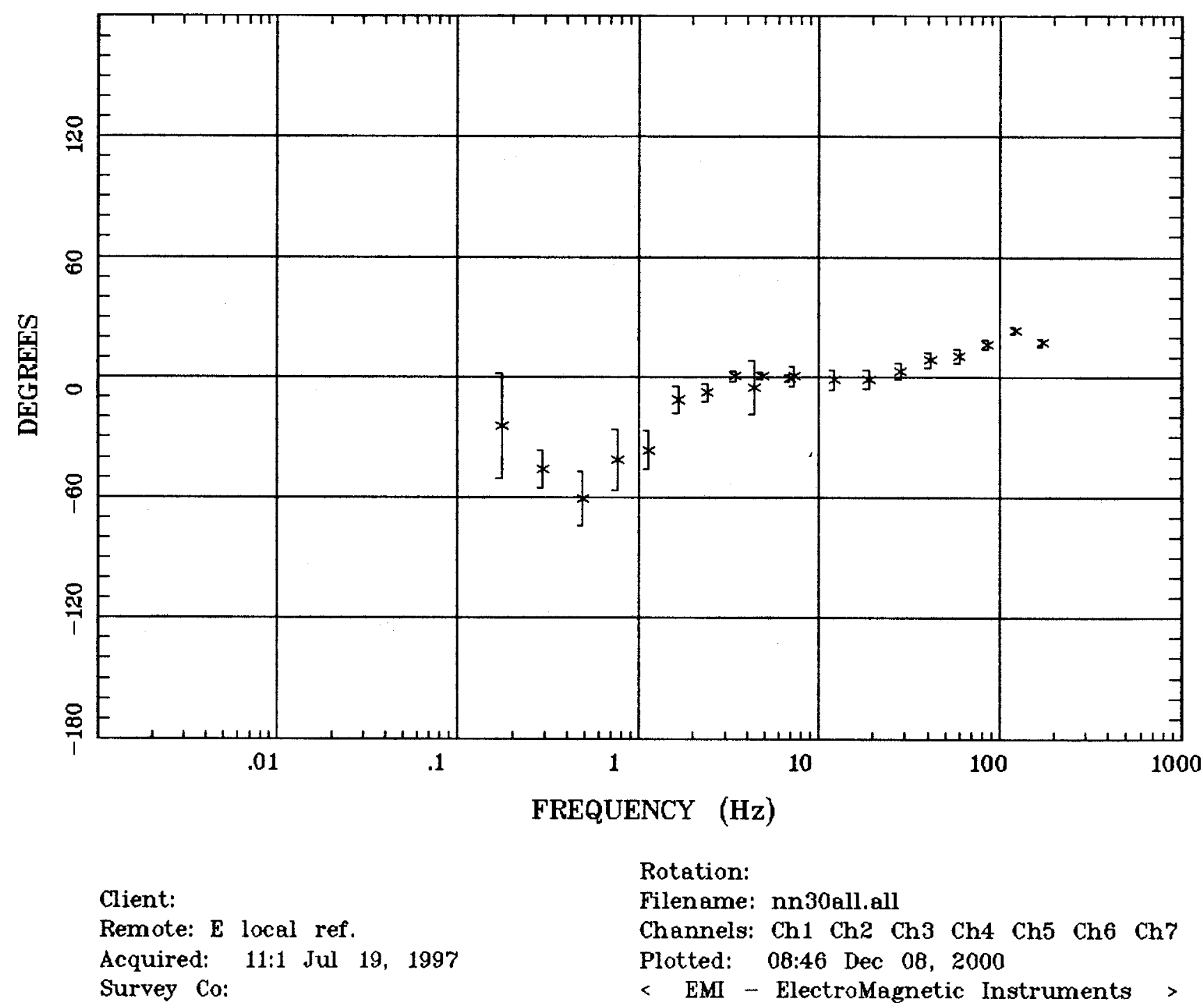




\section{Station 30}

HzHx.x Coh HzHy.o

Indian Creek, Shoshone Mts.

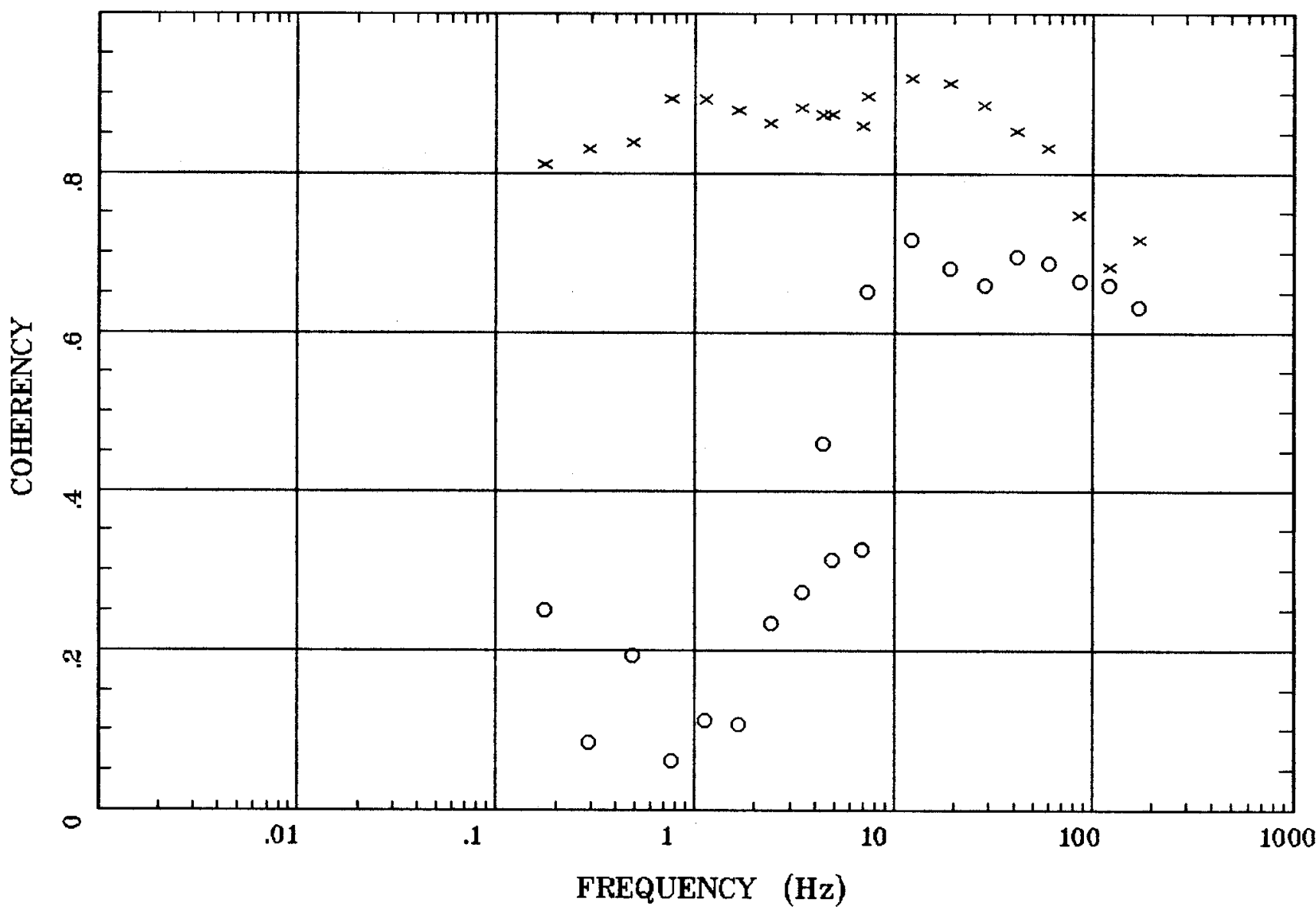

Client:

Remote: E local ref.

Acquired: 11:1 Jul 19, 1997 Survey Co:
Rotation:

Filename: nn30all.all

Channels: Ch1 Ch2 Ch3 ch4 Ch5 ch6 Ch7

Plotted: 08:46 Dec 08, 2000

< EMI - ElectroMagnetic Instruments 
APPARENT RESISTIVITY

Indian Creek, Shoshone Mts.

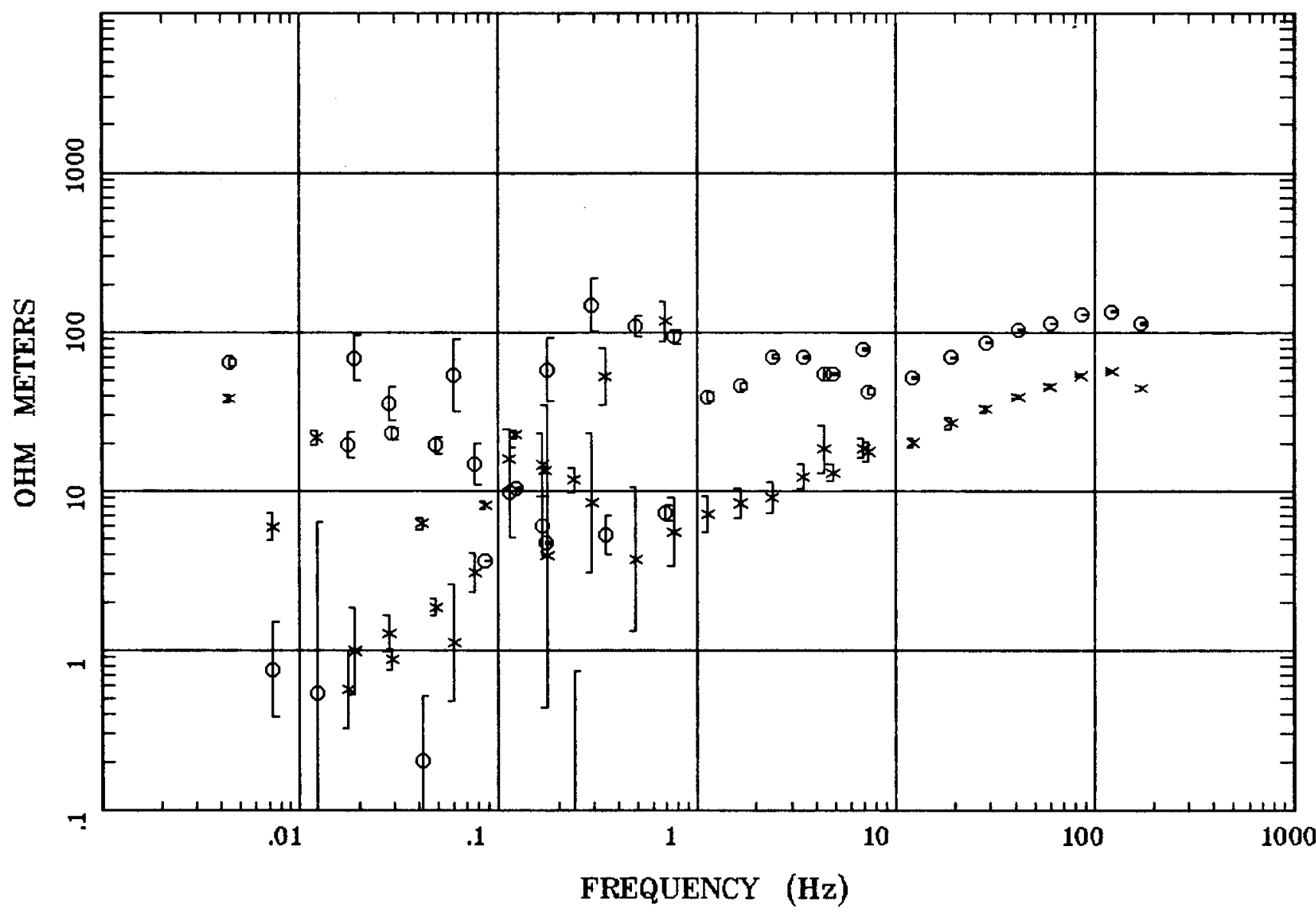

Client:

Remote: E local ref.

Acquired: 18:2 Jul 19, 1997

Survey Co:
Rotation:

Filename: nn31.all

Channels: Ch1 Ch2 Ch3 Ch4 Ch5 Ch6 Ch7

Plotted: 08:47 Dec 08, 2000

< EMI - ElectroMagnetic Instruments 
Indian Creek, Shoshone Mts.

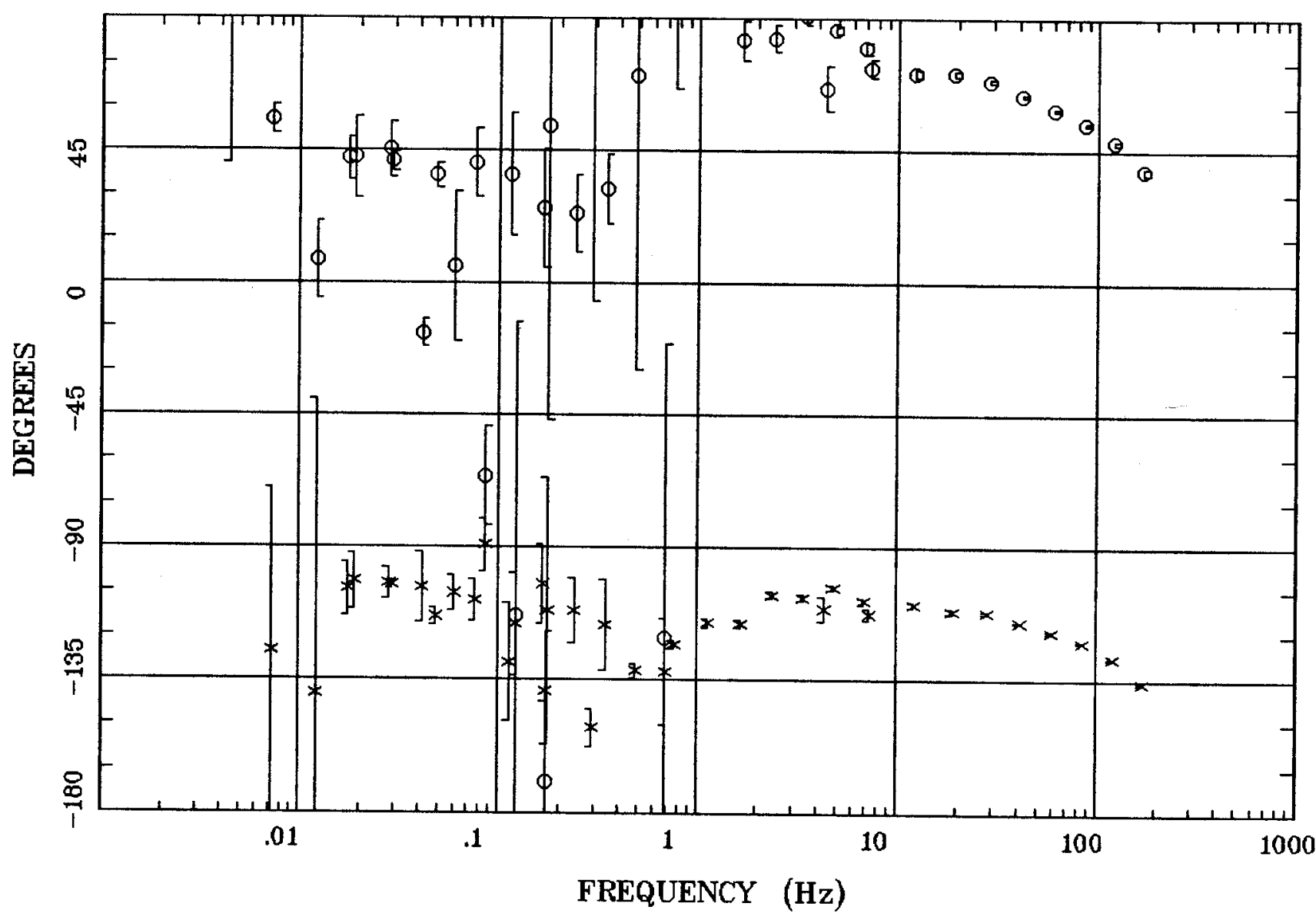

Client:

Remote: E local ref.

Acquired: 18:2 Jul 19, 1997 Survey Co:
Rotation:

Filename: nn31.all

Channels: Ch1 Ch2 Ch3 Ch4 Ch5 Ch6 Ch7

Plotted: 08:47 Dec 08, 2000

<EMI - ElectroMagnetic Instruments > 
Indian Creek, Shoshone Mts.

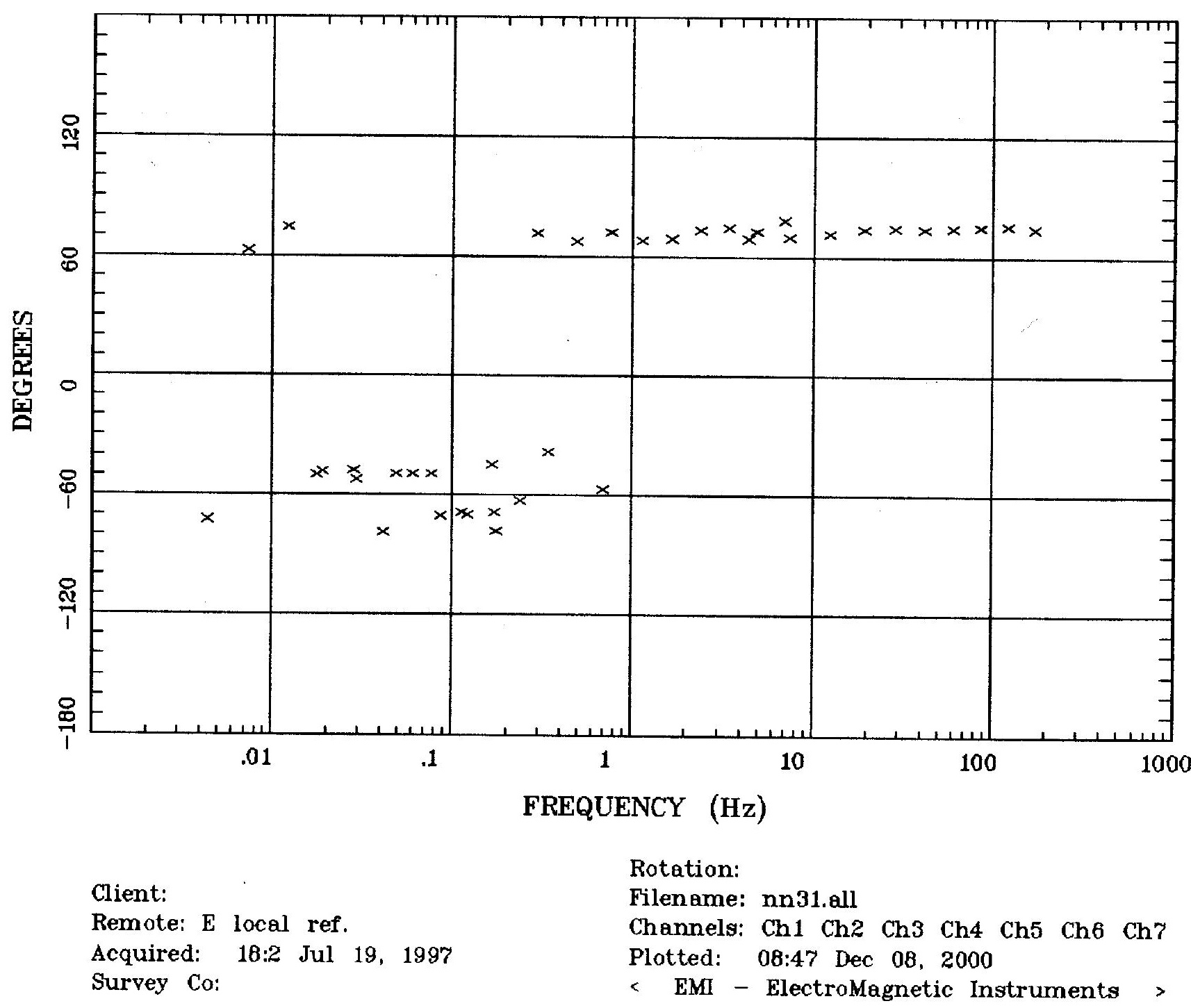




\section{Station 31}

IMPEDANCE SKEW

Indian Creek, Shoshone Mts.

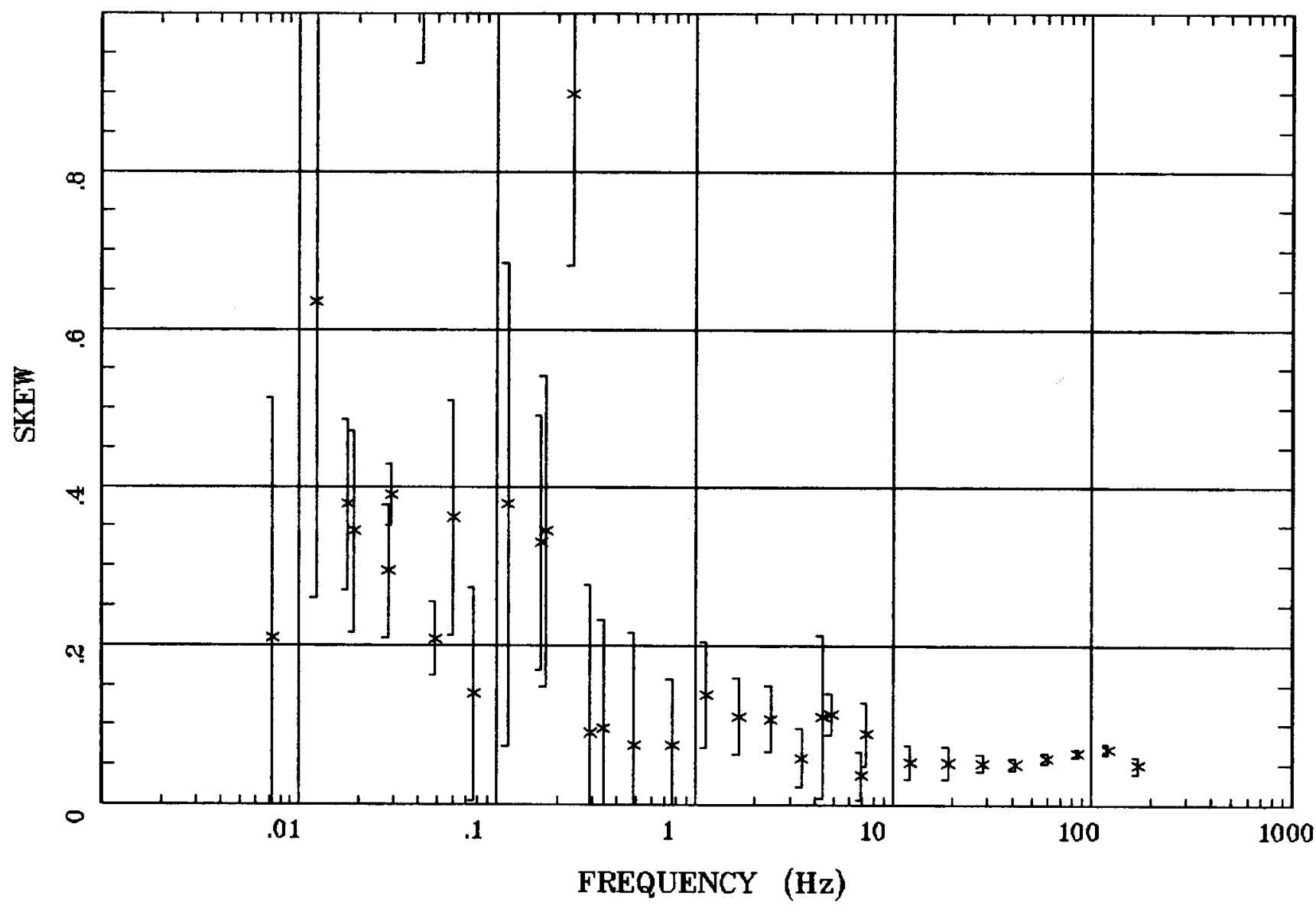

Client:

Rotation:

Remote: E local ref.

Filename: nn31.all

Acquired: 18:2 Jul 19, 1997

Channels: Ch1 Ch2 Ch3 Ch4 Ch5 Ch6 Ch7

Survey $\mathrm{Co}$

Plotted: 08:47 Dec 08, 2000

< EMI - ElectroMagnetic Instruments 
Station 31

E MULT Coh.

Indian Creek, Shoshone Mts.

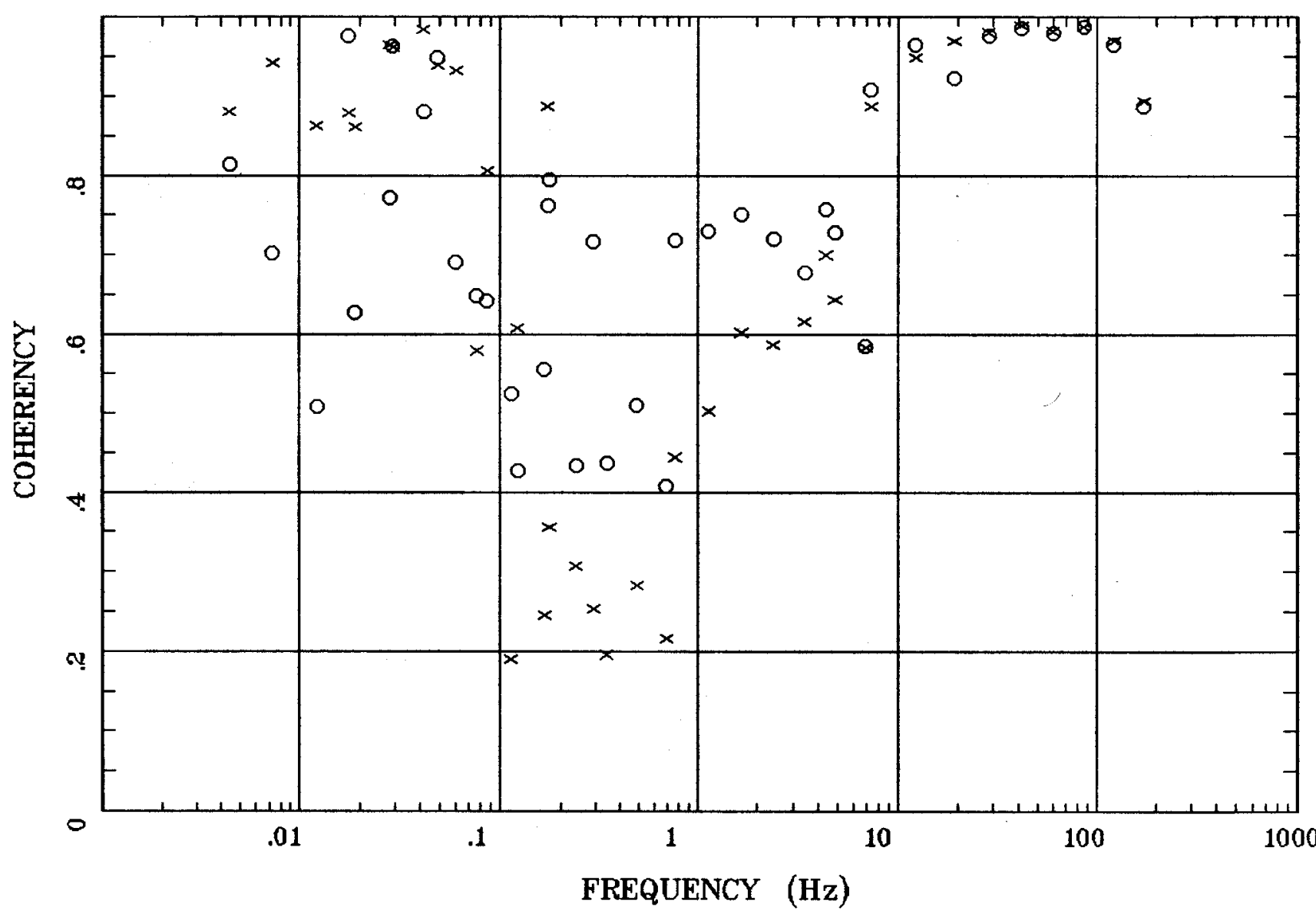

Client:

Remote: E local ref. Acquired: 18:2 Jul 19, 1997 Survey Co:
Rotation:

Filename: nn31.all

Channels: Ch1 Ch2 Ch3 Ch4 Ch5 Ch6 Ch7 Plotted: 08:47 Dec 08, 2000

< EMI - ElectroMagnetic Instruments 
Indian Creek, Shoshone Mts.

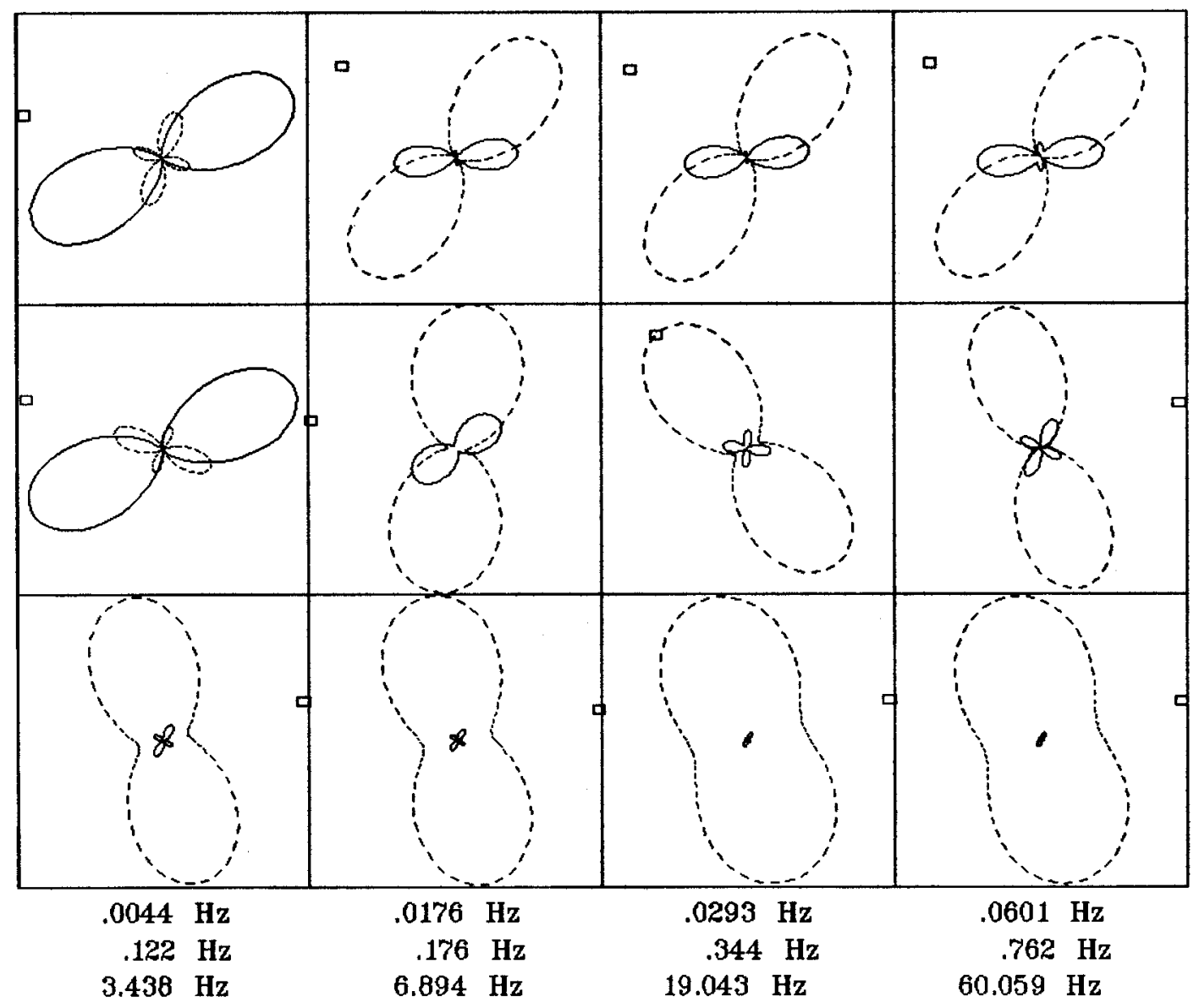

Client:

Remote: E local ref.

Acquired: 18:2 Jul 19, 1997 Survey Co:
Rotation:

Filename: nn31.all

Channels: Ch1 Ch2 Ch3 Ch4 Ch5 Ch6 Ch7 Plotted: 08:47 Dec 08, 2000

$<$ EMI - ElectroMagnetic Instruments 
TIPPER MAGNITUDE

Indian Creek, Shoshone Mts.

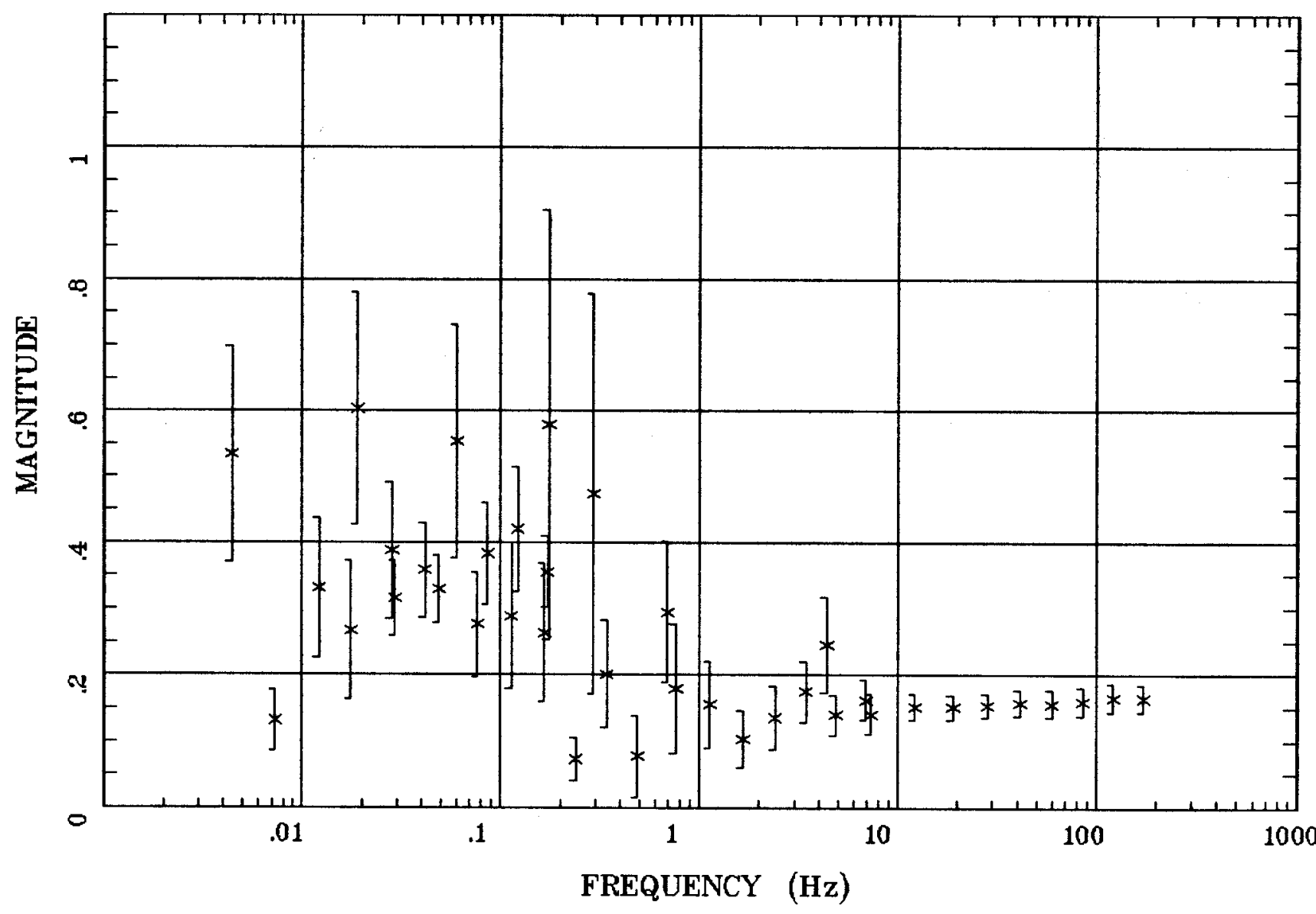

Client:

Remote: E local ref.

Acquired: 18:2 Jul 19, 1997 Survey Co:
Rotation:

Filename: nn31.all

Channels: Ch1 Ch2 Ch3 Ch4 Ch5 Ch6 Ch?

Plotted: 08:47 Dec 08, 2000

$<$ EMI - ElectroMagnetic Instruments > 


\section{Station 31}

TIPPER STRIKE

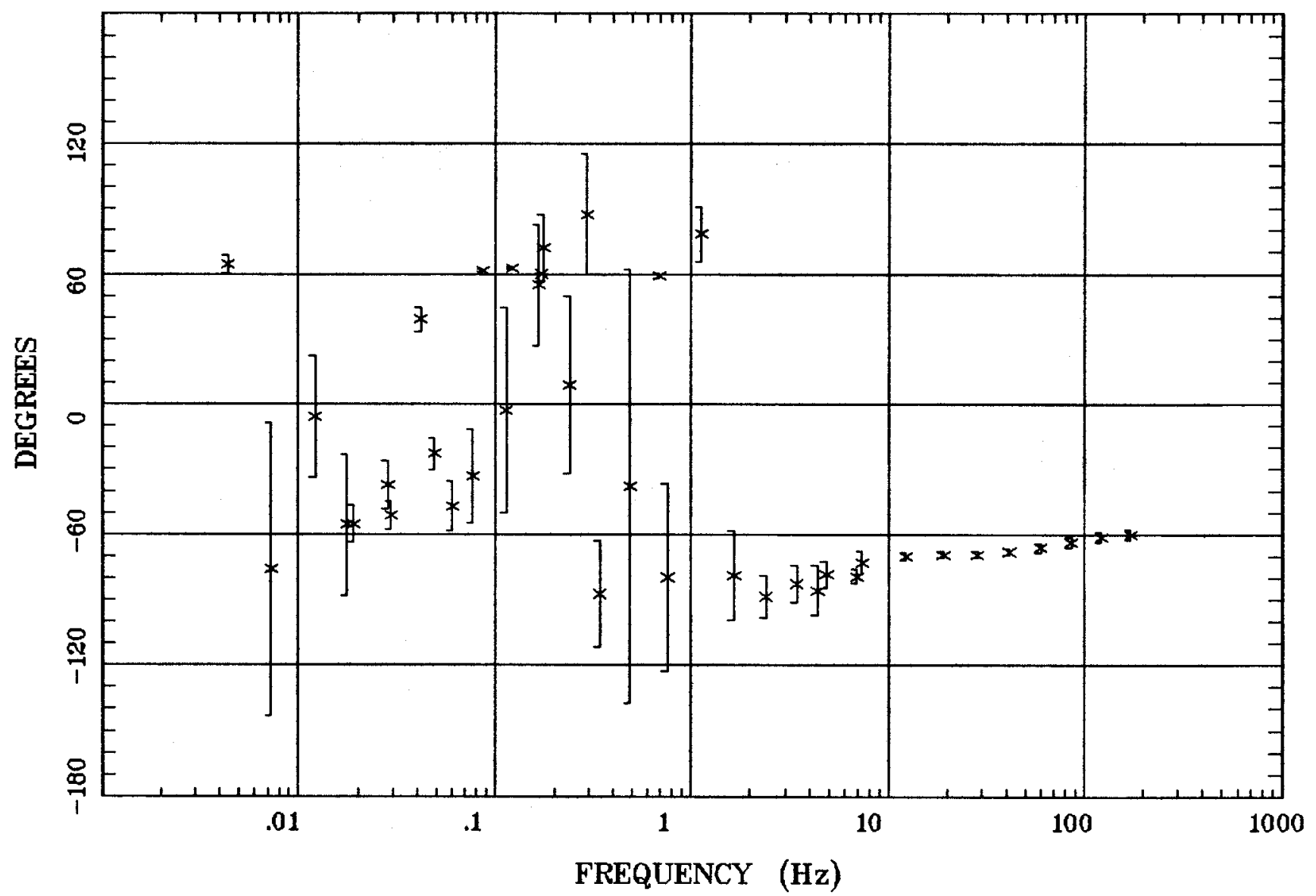

Client:

Remote: E local ref.

Acquired: $18: 2$ Jul 19, 1997

Survey Ca:
Indian Creek, Shoshone Mts.

\section{Rotation:}

Filename: nn31.all

Channels: Ch1 Ch2 Ch3 ch4 Ch5 Ch6 Ch7

Plotted: 08:47 Dec 08, 2000

< EMI - ElectroMagnetic Instruments > 
HzHx.x Coh HzHy.o

Indian Creek, Shoshone Mts.

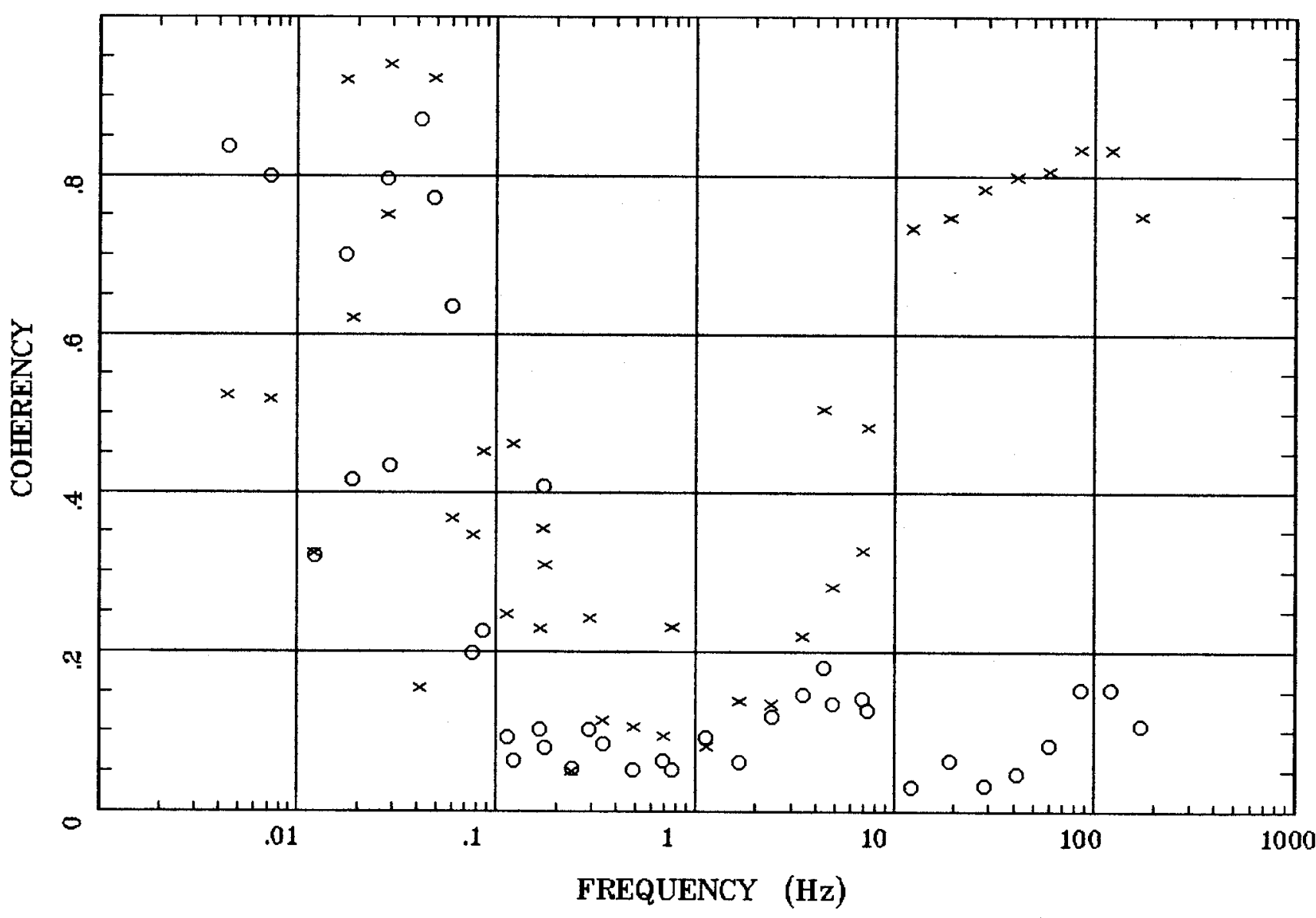

Client:

Remote: E local ref.

Acquired: 18:2 Jul 19, 1997 Survey Co:
Rotation:

Filename: nn31.all

Channels: Ch1 Ch2 Ch3 Ch4 Ch5 Ch6 Ch7

Plotted: 08:47 Dec 08, 2000

$<$ EMI - ElectroMagnetic Instruments > 
APPARENT RESISTIVITY

...Indian Creek...

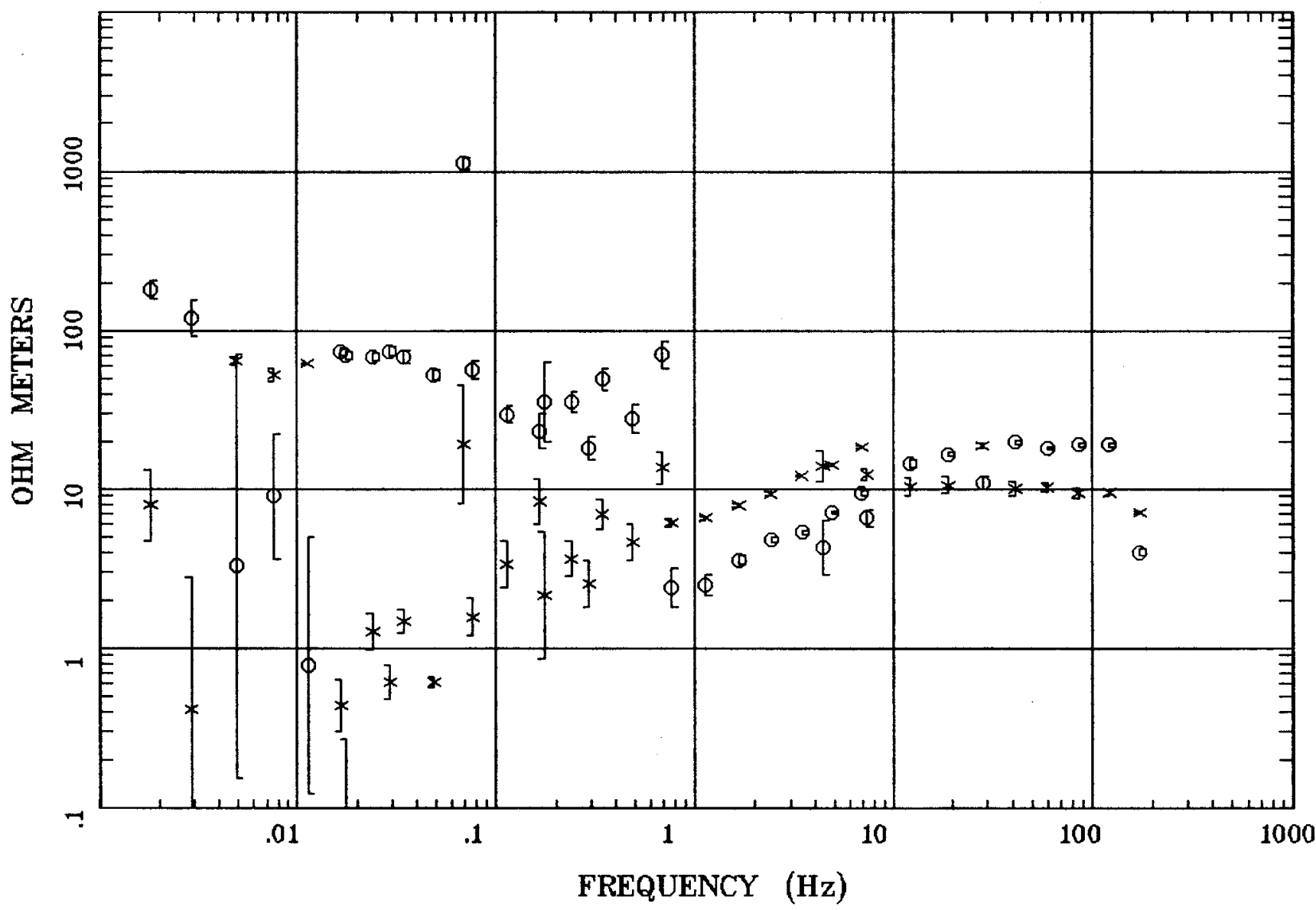

Client:

Remote: E local ref.

Acquired: 15:1 Jul 22, 1997

Survey Co:
Rotation:

Filename: nn35.all

Channels: Ch6 Ch7 Ch8 Ch9 Ch10Ch1 Ch2

Plotted: 08:48 Dec 08, 2000

< EMI - ElectroMagnetic Instruments > 
IMPEDANCE PHASE

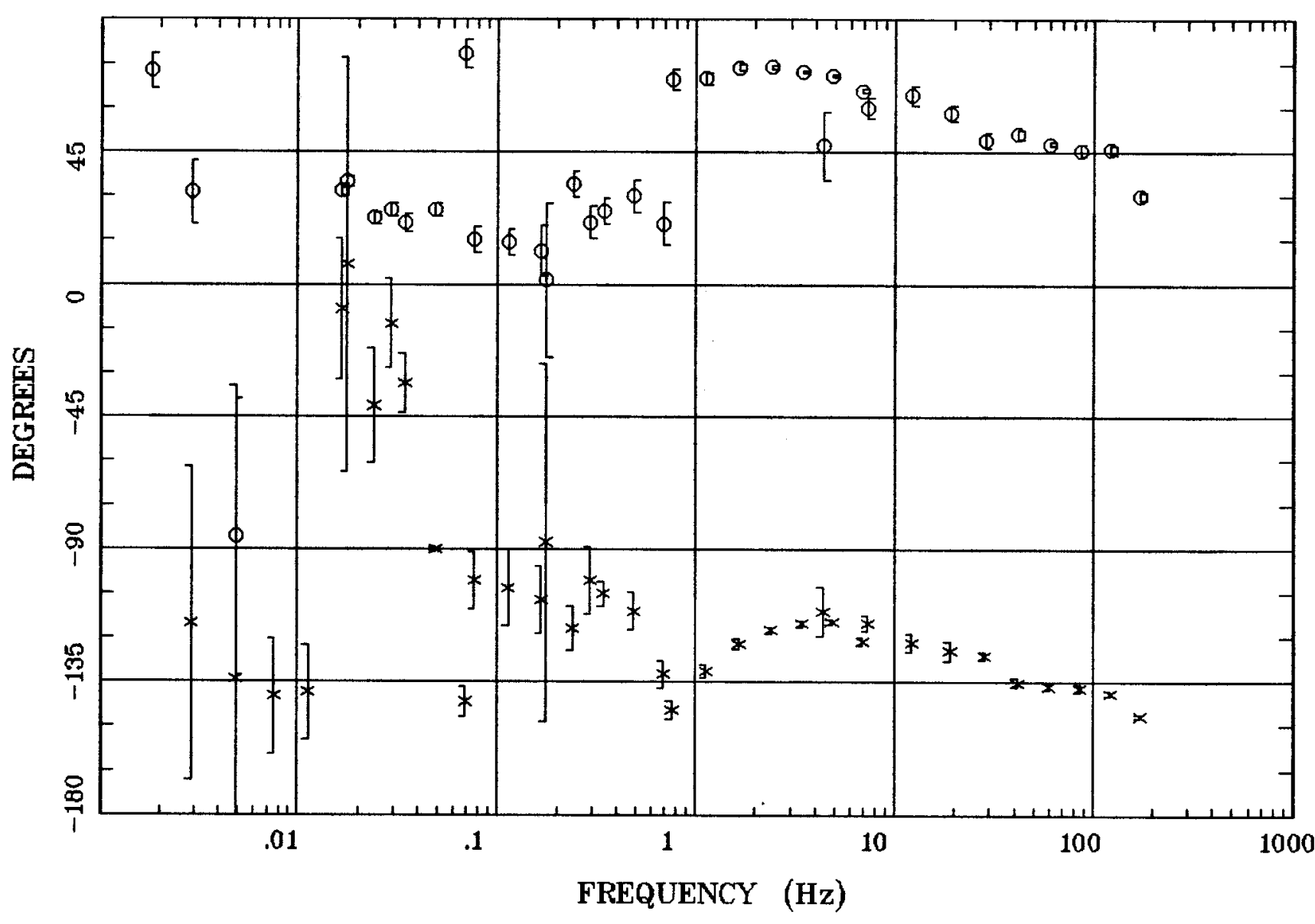

Client:

Remote: E local ref.

Acquired: 15:1 Jul 22, 1997

Survey Co:

\section{...Indian Creek...}


...Indian Creek...

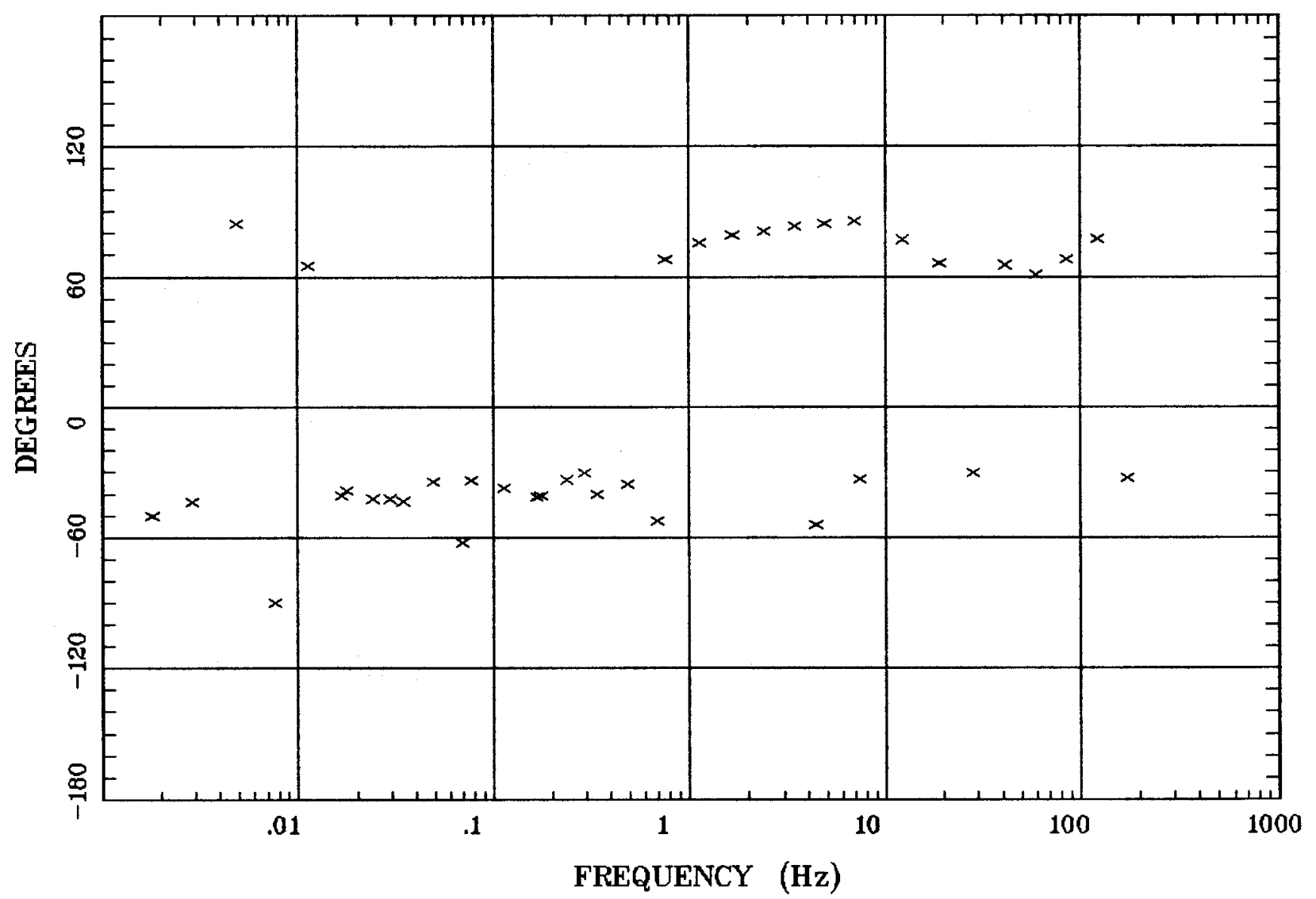

Client:

Remote: E lacal ref.

Acquired: 15:1 Jul 22, 1997

Rotation:

Filename: nn35.all

Channels: Ch6 Ch7 Ch8 Ch9 Ch10Ch1 Ch2

Plotted: 08:48 Dec 08, 2000

Survey Co:

$<$ EMI - ElectroMagnetic Instruments 
Station 35

IMPEDANCE SKEW

...Indian Creek...

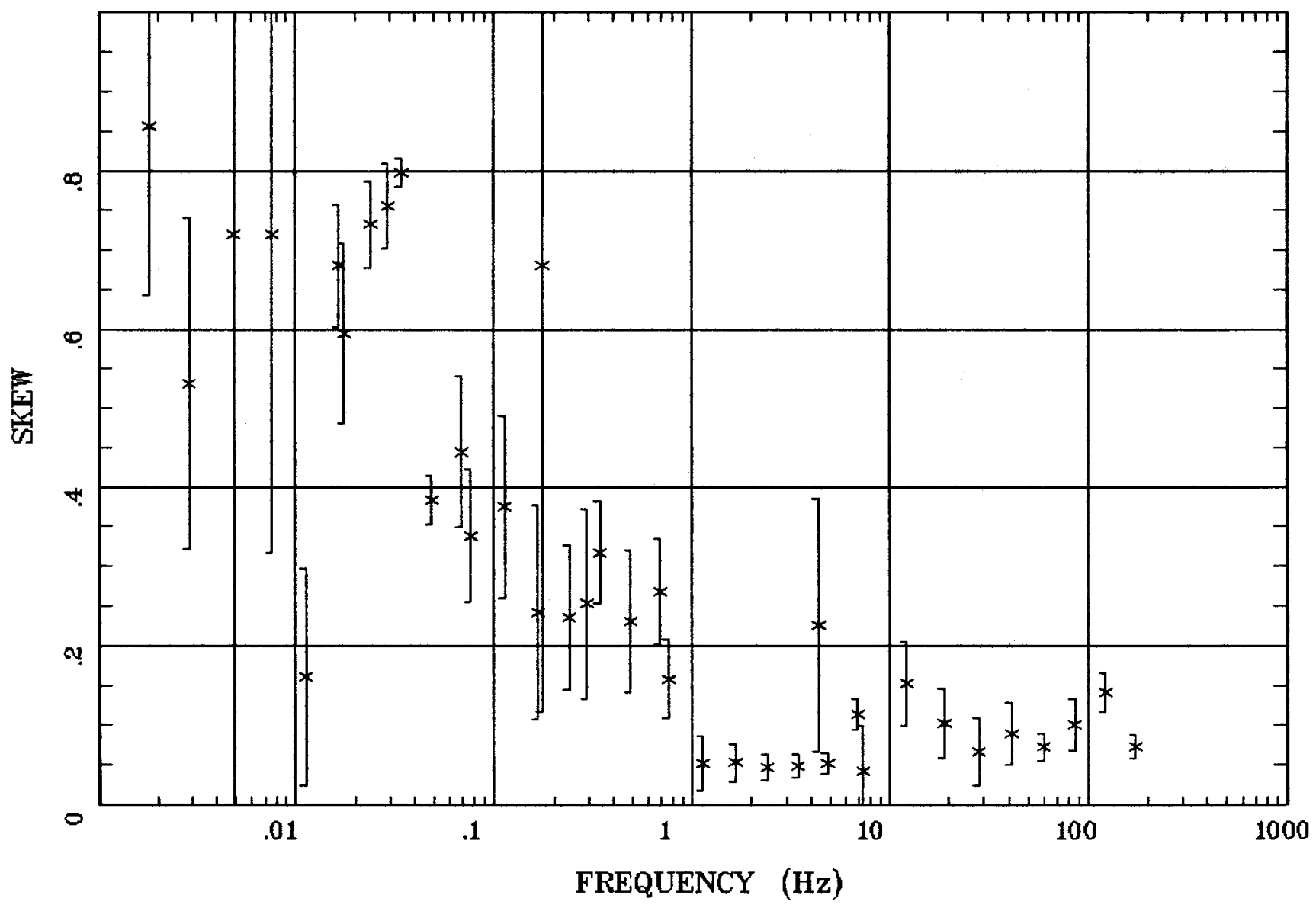

Client:

Remote: E local ref.

Acquired: 15:1 Jul 22, 1997

Survey Co:

Rotation:

Fileneme: nn35.all

Channels: Ch6 Ch7 Ch8 Ch9 Ch10Ch1 Ch2

Plotted: 08:48 Dec 08, 2000

< EMI - ElectroMagnetic Instruments > 
Station 35

E MULT Coh.

...Indian Creek...

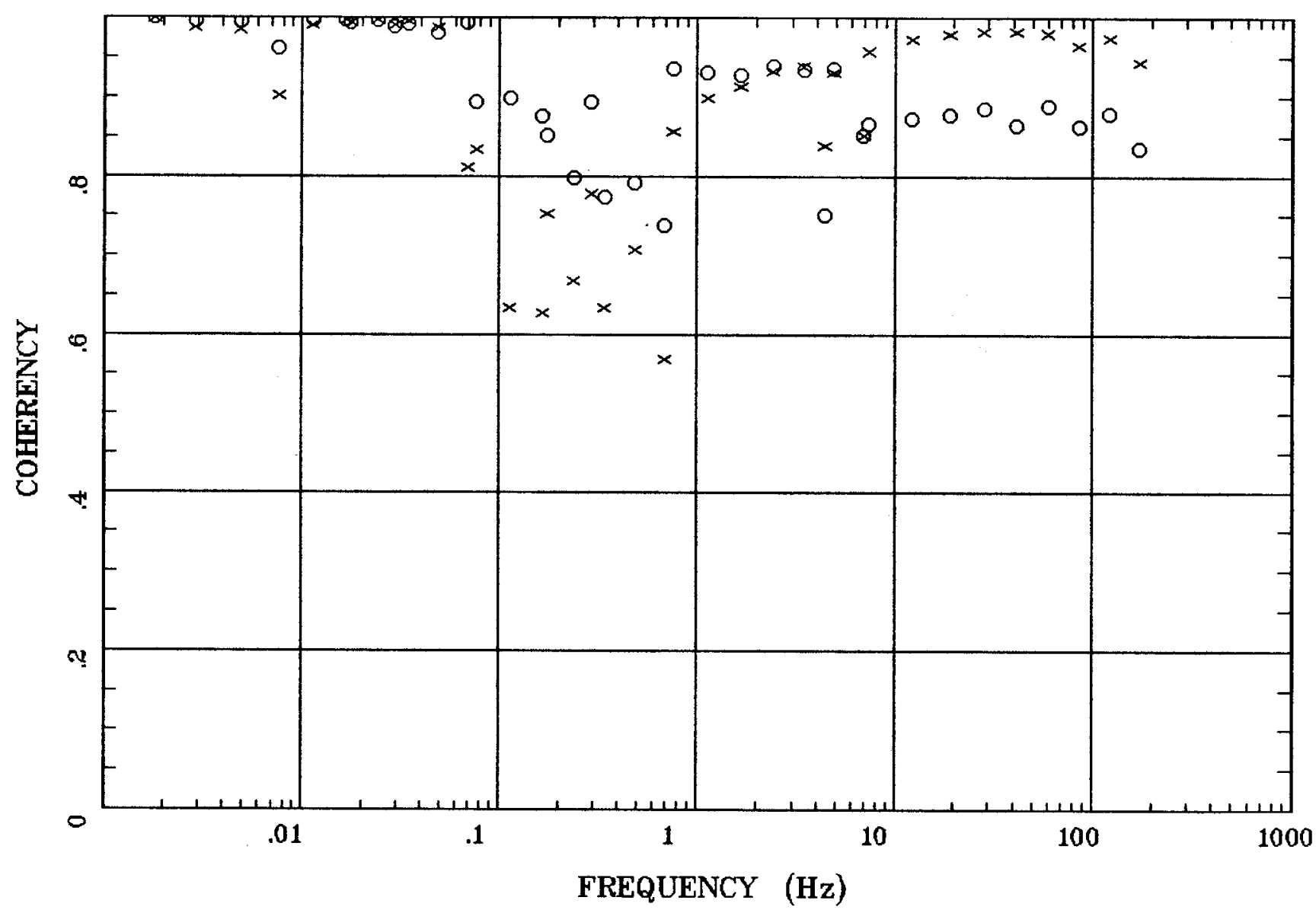

Client:

Rotation:

Remote: E local ref.

Acquired: 15:1 Jul 22, 1997

Filename: nn35.all

Survey Co:

Channels: Ch6 Ch7 Ch8 Ch9 Ch10Ch1 Ch2

Plotted: 08:48 Dec 08, 2000

$<$ EMI - ElectroMagnetic Instruments 


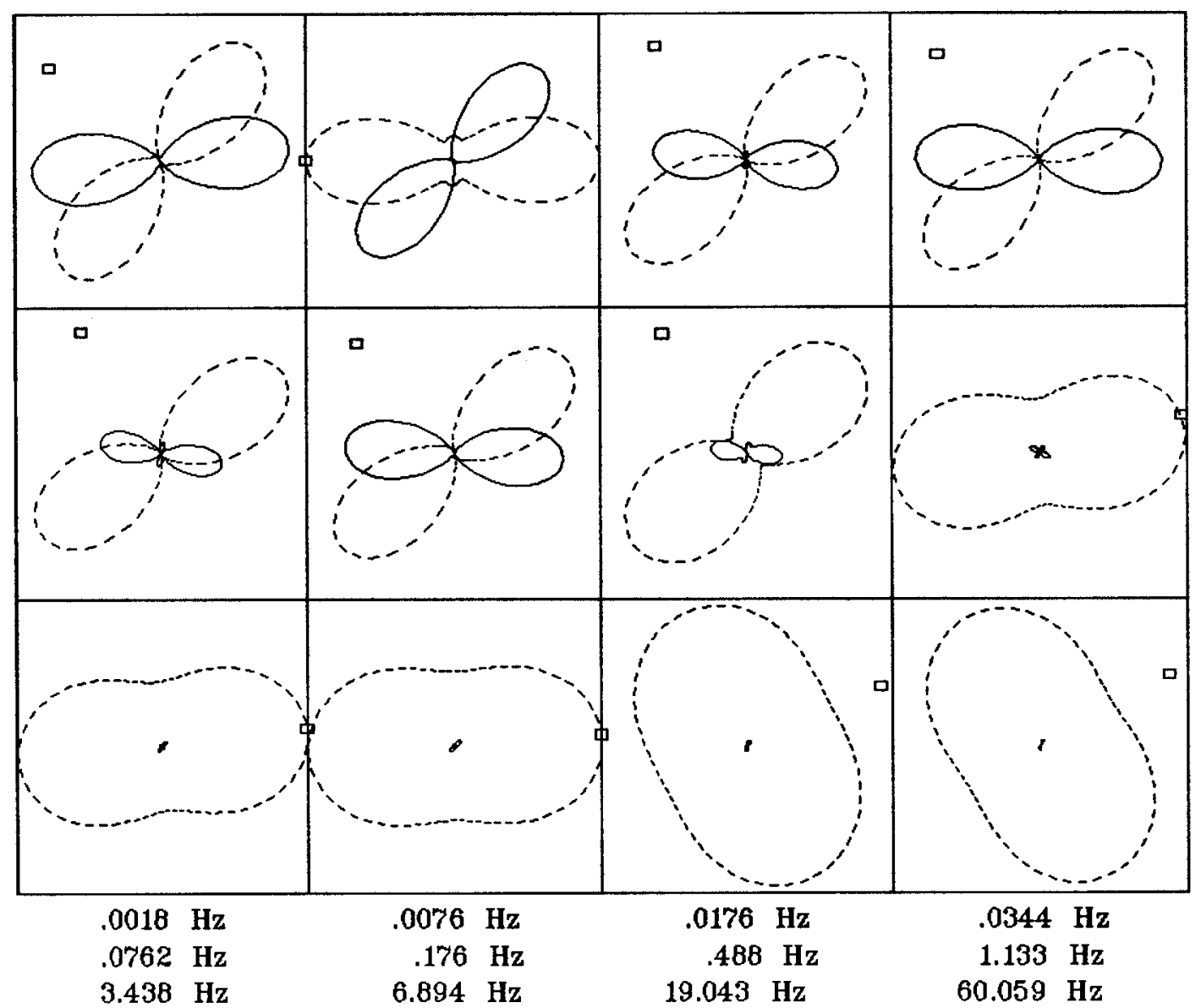

\section{Client:}

Remote: E local ref.

Acquired: 15:1 Jul 22, 1997 Survey Co:
Rotation:

Filename: nn35.all

Channels: Ch6 Ch7 Ch8 Ch9 Ch10Ch1 Ch2

Plotted: 08:48 Dec 08, 2000

< EMI - ElectroMagnetic Instruments > 
TIPPER MAGNITUDE

...Indian Creek...

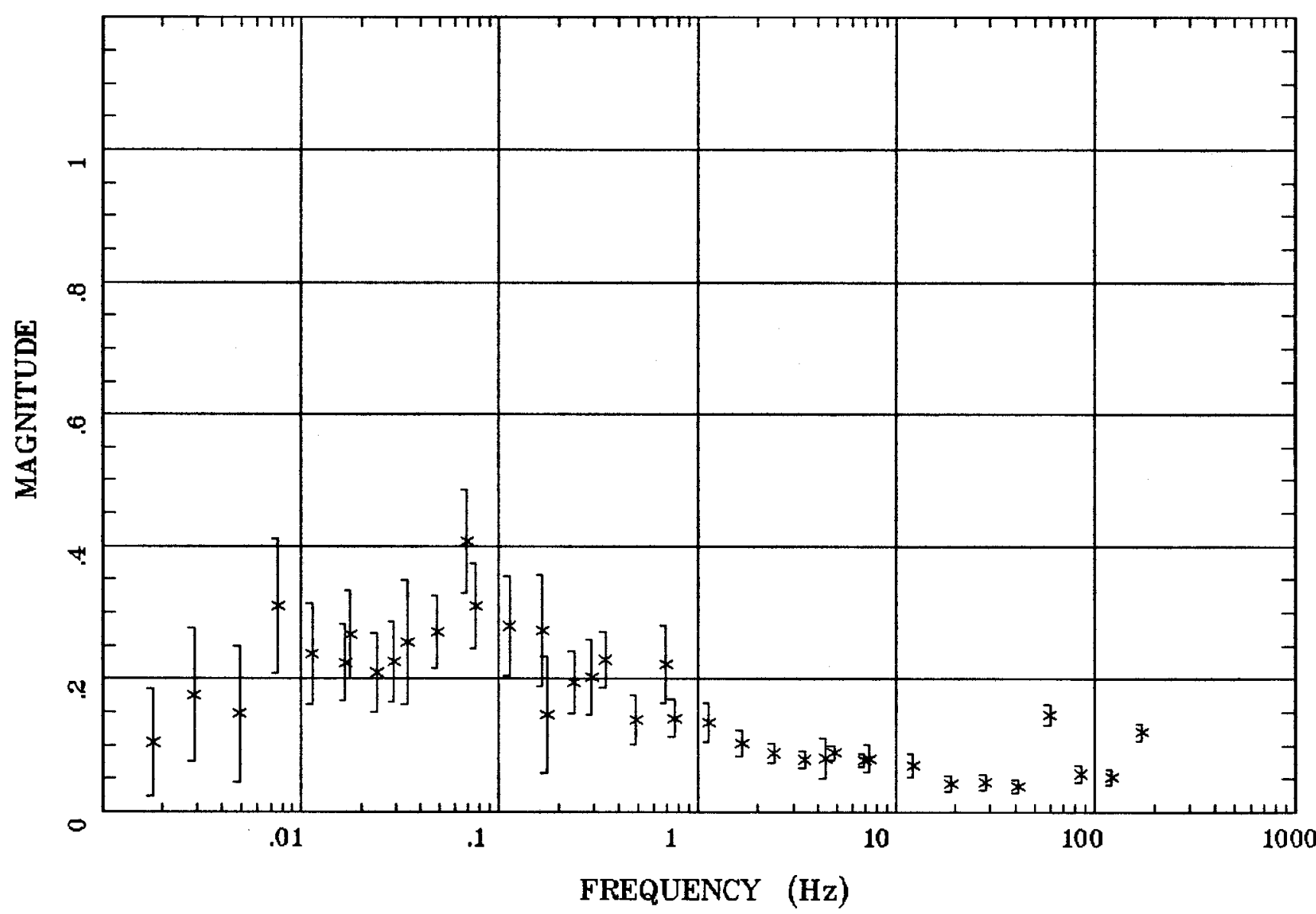

Client:

Remote: E local ref.

Acquired: 15:1 Jul 22, 1997

Survey Co:
Rotation:

Filename: nn35.all

Channels: Ch6 Ch' Ch8 Ch9 Ch10Ch1 Ch2

Plotted: 08:48 Dec 08, 2000

< EMI - ElectroMagnetic Instruments 
Station 35

TIPPER STRIKE

...Indian Creek...

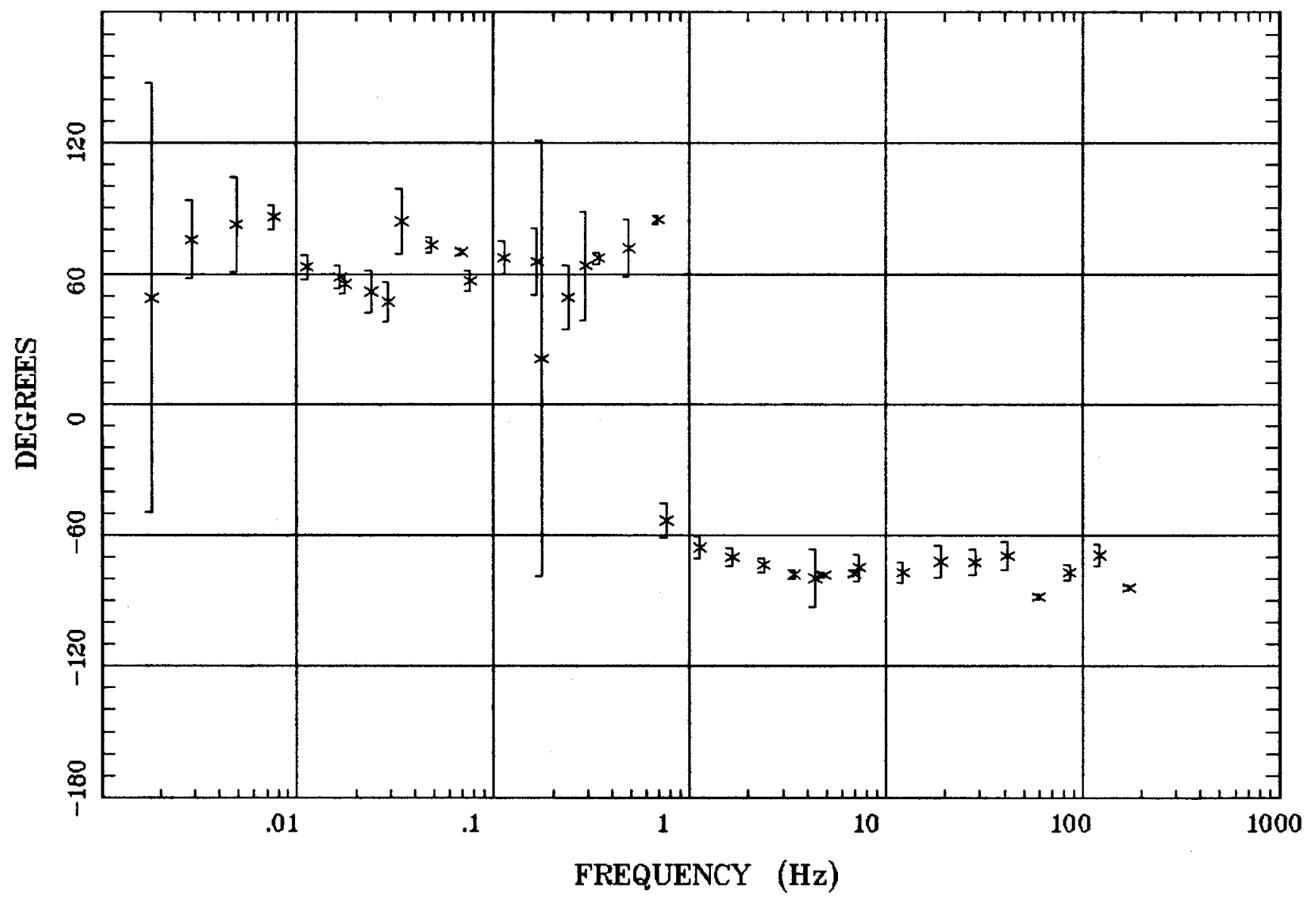

Client:

Remote: E local ref.

Acquired: 15:1 Jul 22, 1997

Survey Co:

Rotation

Filename: nn35.all

Channels: Ch6 Ch7 Ch8 Ch9 Ch10Ch1 Ch2

Plotted: 08:48 Dec 08, 2000

< EMI - ElectroMagnetic Instruments > 


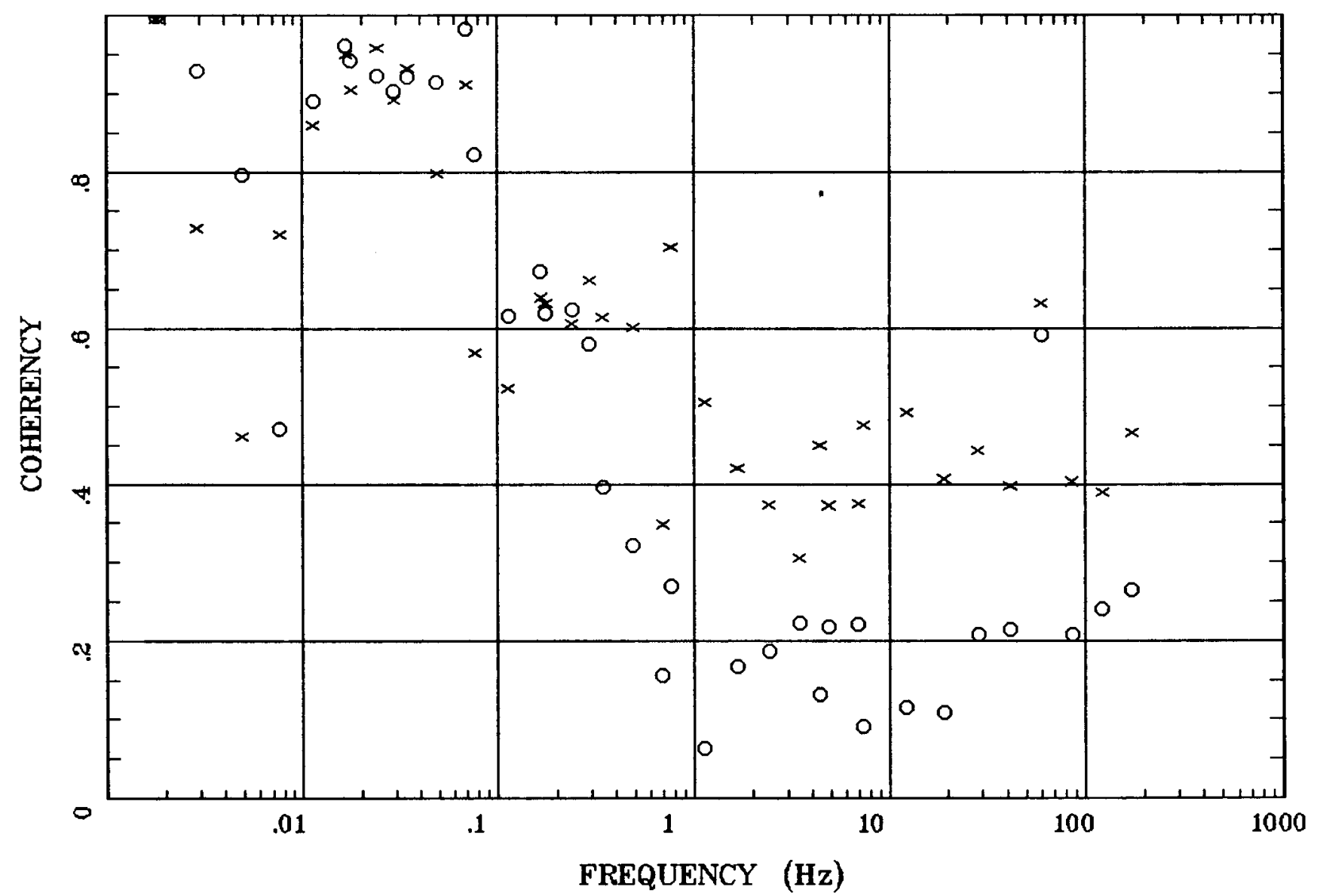

Client:

Remote: E local ref.

Acquired: 15:1 Jul 22, 1997

Rotation:

Filename: nn35.all

Survey $\mathrm{Co}$ :

Channels: Ch6 Ch7 Ch8 Ch9 Ch10Ch1 Ch2

Plotted: 08:48 Dec 08, 2000

< EMI - ElectroMagnetic Instruments > 